\title{
15. LATE OLIGOCENE THROUGH EARLY PLEISTOCENE CALCAREOUS NANNOFOSSILS FROM WESTERN EQUATORIAL INDIAN OCEAN (LEG 115) ${ }^{1}$
}

\author{
Domenico Rio, ${ }^{2}$ Eliana Fornaciari, ${ }^{2}$ and Isabella Raffi ${ }^{3}$
}

\begin{abstract}
The chronostratigraphy, the calcareous nannofossil biochronology, and the biostratigraphy of the Miocene and Pliocene sediments retrieved during Leg 115 in the equatorial western Indian Ocean are presented and discussed. Most of the zonal boundaries of the standard 1971 zonation of Martini and the 1973 zonation of Bukry are easily recognized in these low-latitude sediments. We also comment on the secondary events that are proposed in the literature to improve the biostratigraphic resolution provided by the standard zonations.

The study of calcareous nannofossil biostratigraphy and taphonomy of sequences from the Northern Mascarene Plateau area, which was drilled to investigate the Neogene history of carbonate flux and dissolution, indicate that the accumulation of carbonates in this area results from a complex interplay among carbonate bioproductivity, carbonate removal by chemical dissolution and mechanical erosion, and carbonate addition by mass and current transport. In spite of these drawbacks, major changes and trends in carbonate accumulation can be recognized, most of which, if not all, correlate with major steps in the evolution of the Neogene climatic system.
\end{abstract}

\section{INTRODUCTION}

Ocean Drilling Program (ODP) Leg 115 in the western equatorial Indian Ocean was the first of a nine-leg program exploring the Indian Ocean. The scientific objectives of Leg 115 were to study the Réunion hotspot volcanism and the Neogene stratigraphy and paleoenvironment of the western equatorial Indian Ocean, with special emphasis on the history of carbonate production and dissolution.

Advanced hydraulic piston corer (APC), extended core barrel (XCB), and rotary core barrel ( $\mathrm{RCB}$ ) drilling techniques were used, and 22 holes were drilled at 12 sites (Fig. 1 and Table 1). The sites can be divided into four groups according to location and scientific objectives:

1. Southern Mascarene Plateau (Nazareth Bank): Sites 705 and 706 , with mainly basement objectives.

2. Northern Mascarene Plateau and Madingley Rise: Sites 707 through 711. These sites represent a depth-dependent core transect (carbonate bathymetric transect [CBT]) drilled to investigate the history of carbonate flux and dissolution during the Neogene. They were drilled in a narrowly confined geographic area on oceanic crust of similar age (basal Eocene; Schlich, 1982 ) at different water depths (from 1551 to $4438 \mathrm{~m}$ ) to ensure that the surface-water production of skeletal material is similar and to ensure the crossing of critical bottom-water chemical boundaries (the carbonate compensation depth [CCD] and lysocline).

3. Chagos: Sites 712 and 713 , mainly with basement objectives.

4. Maldives: Sites 714,715 , and 716 , with paleoclimatic and basement (Site 715) objectives.

A total of $3058 \mathrm{~m}$ of cored material was recovered. Oceanic basaltic basement was reached and penetrated at four sites (706,

\footnotetext{
${ }^{1}$ Duncan, R. A., Backman, J., Peterson, L. C., et al., 1990. Proc. ODP, Sci. Results, 115: College Station, TX (Ocean Drilling Program).

2 Dipartimento di Geologia Paleontologia e Geofisica, Università di Padova, Via Giotto, 35137 Padova, Italy.

${ }^{3}$ Istituto di Geologia, Università di Parma, Via delle Scienze, 43100 Parma,
}

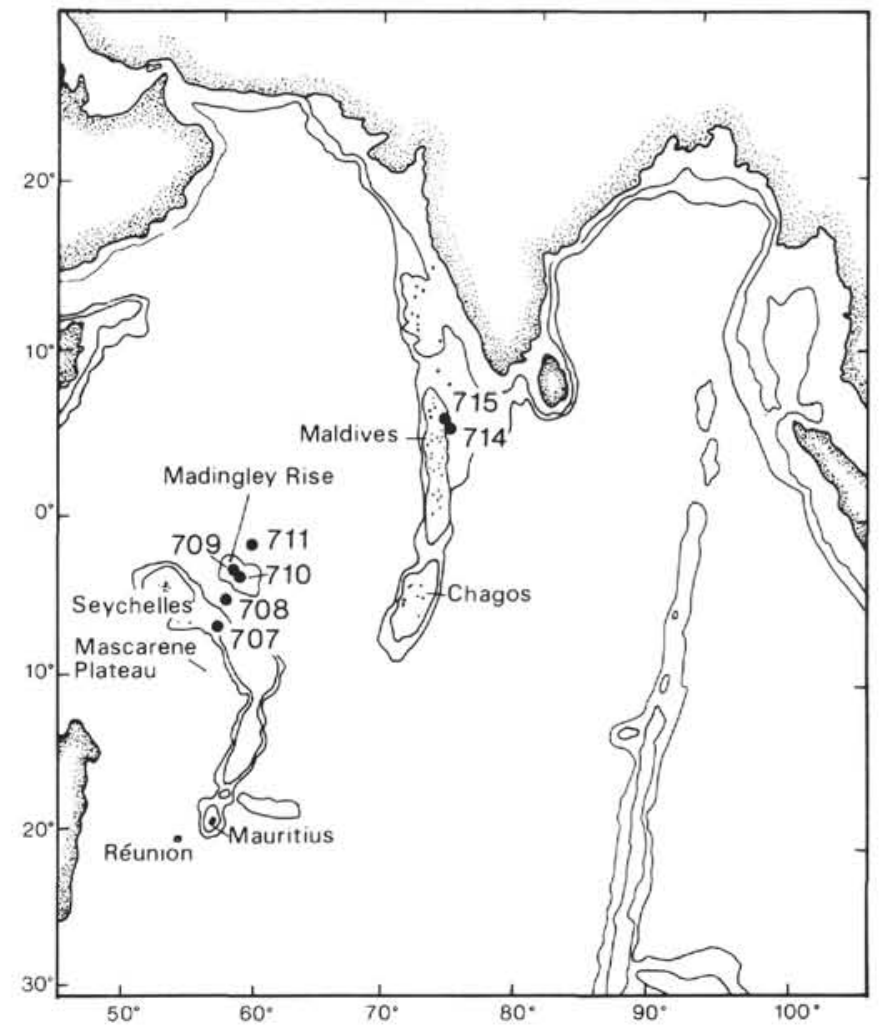

Figure 1. Location map for Leg 115 sites.

707, 713, and 715; Backman, Duncan, et al., 1988). The sediments recovered range, stratigraphically, from the early Paleocene to Pleistocene.

This study describes the latest Oligocene to the earliest Pleistocene calcareous nannofossil assemblages. For calcareous nannofossils in the underlying Paleogene and the overlying Pleistocene sediments of Leg 115, see Okada (this volume). This study will deal with (1) the biostratigraphic classification and age assignment of the recovered Miocene and Pliocene sections; (2) a 
Table 1. Summary of Leg 115 sites and holes.

\begin{tabular}{ccccc}
\hline & \multicolumn{2}{c}{ Position } & Water depth & $\begin{array}{c}\text { Number } \\
\text { of holes }\end{array}$ \\
\cline { 2 - 3 } Site & Latitude & Longitude & & 1 \\
\hline 705 & $13^{\circ} 10.00^{\prime} \mathrm{S}$ & $61^{\circ} 22.27^{\prime} \mathrm{E}$ & 2318.0 & 1 \\
706 & $13^{\circ} 06.86^{\prime} \mathrm{S}$ & $61^{\circ} 22.27^{\prime} \mathrm{E}$ & 2517.0 & 3 \\
707 & $07^{\circ} 32.72^{\prime} \mathrm{S}$ & $59^{\circ} 01.01^{\prime} \mathrm{E}$ & 1551.9 & 3 \\
708 & $05^{\circ} 27.23^{\prime} \mathrm{S}$ & $59^{\circ} 56.63^{\prime} \mathrm{E}$ & 4107.0 & 1 \\
709 & $03^{\circ} 54.72^{\prime} \mathrm{S}$ & $60^{\circ} 33.16^{\prime} \mathrm{E}$ & $3046.9-3048.7$ & 3 \\
710 & $04^{\circ} 18.69^{\prime} \mathrm{S}$ & $60^{\circ} 48.76^{\prime} \mathrm{E}$ & 3822.5 & 2 \\
711 & $02^{\circ} 44.46^{\prime} \mathrm{S}$ & $61^{\circ} 09.75^{\prime} \mathrm{E}$ & 4438.7 & 2 \\
712 & $04^{\circ} 12.99^{\prime} \mathrm{S}$ & $73^{\circ} 24.38^{\prime} \mathrm{E}$ & 2902.9 & 1 \\
713 & $04^{\circ} 11.58^{\prime} \mathrm{S}$ & $73^{\circ} 23.65^{\prime} \mathrm{E}$ & 2920.3 & 1 \\
714 & $05^{\circ} 03.69^{\prime} \mathrm{N}$ & $73^{\circ} 46.98^{\prime} \mathrm{E}$ & 2042.0 & 2 \\
715 & $05^{\circ} 04.89^{\prime} \mathrm{N}$ & $73^{\circ} 49.88^{\prime} \mathrm{E}$ & 2272.8 & 1 \\
716 & $04^{\circ} 56.00^{\prime} \mathrm{N}$ & $73^{\circ} 17.01^{\prime} \mathrm{E}$ & 543.8 & 2 \\
\hline
\end{tabular}

discussion of the calcareous nannofossil Miocene and Pliocene biostratigraphy in the equatorial Indian Ocean; (3) a discussion of some aspects of the Neogene calcareous nannofossil biochronology; (4) the improvement of the biostratigraphic resolution in the Miocene obtainable by means of calcareous nannofossils; and (5) a discussion of hiatuses and of some aspects of the history of carbonate accumulation and dissolution as evidenced by the study of calcareous nannofossils.

More detailed information on the distribution patterns of late Oligocene-Miocene stratigraphically indicative calcareous nannofossils is reported in Fornaciari et al. (this volume), who have collected quantitative data from many of the samples used in this study.

\section{METHODS}

The nannofossil assemblages were described from smear slides prepared for each sample from unprocessed material. The slides were examined with a light microscope at approximately $\times 1400$ magnification using cross-polarized and transmitted light. A total of more than 2000 samples was examined. However, we plotted only selected samples (1064) in the range charts.

\section{Abundance}

Abundance estimates of the nannofossils in the slides were made on optimum density areas of the slides, that is, areas where most of the field was covered with sample material without appreciable piling of specimens or sample material. The abundance code translates as follows:

$$
\begin{array}{ll}
\mathrm{A} \text { (abundant) }= & \text { usually more than } 10 \text { specimens occurring } \\
& \text { per field; } \\
\mathrm{C} \text { (common) }= & 1-10 \text { specimens per field; } \\
\mathrm{F} \text { (few) } & =1 \text { specimen per } 1-10 \text { fields; and } \\
\mathrm{R} \text { (rare) } & =1 \text { specimen per more than } 10 \text { fields. }
\end{array}
$$

Abundance estimates of the abundance of individual nannofossil species on the smear slide were obtained by means of the following procedures:

1. To characterize the general makeup of the assemblage, we identified and counted the first 200-300 specimens encountered under a light microscope at approximately $\times 1400$ magnification and calculated the percentage.

2. To check the presence or absence of index species in critical intervals, we continued our observations until a reasonable number of nannofossils had been scanned. This number varied depending on the abundance of marker species and on the overall abundance of nannofossils. In the case of abundant species (i.e., Reticulofenestra pseudoumbilicus), at least 500 specimens were observed, following the procedure of Thierstein et al. (1977) and Rio et al. (1989). In the case of subordinate species (discoasterids, helicoliths, sphenoliths) or rare (i.e., ceratolithids), the presence or absence of an index species was evaluated by observing a fixed number of forms belonging to the group (200300 discoasterids, 100 helicoliths and sphenoliths, and 30-50 ceratolithids; see Backman and Shackleton, 1983, and Rio et al., 1989, for discussion about this counting technique).

Following the procedure agreed on board ship on Leg 115, the abundance of each taxa was tabulated in the range charts according to the following criteria:

$$
\begin{aligned}
& \mathrm{D}=>50 \%, \\
& \mathrm{~A}=10 \%-50 \%, \\
& \mathrm{C}=1 \%-10 \%, \\
& \mathrm{~F}=0.1 \%-1 \%, \text { and } \\
& \mathrm{R}=<0.1 \% .
\end{aligned}
$$

Taxa considered in this chapter are listed in the appendix, where they are arranged alphabetically by generic epithets. Bibliographic references for these species are given in Loeblich and Tappan (1966, 1968, 1969, 1970a, 1970b, 1971, 1973) and Van Heck (1979a, 1979b, 1980a, 1980b, 1981a, 1981b, 1982a, 1982b). Remarks on taxonomic concepts are given in the "Taxonomic Notes" section at the end of this chapter.

\section{Preservation}

One of the main objectives of Leg 115 was to study the history of carbonate flux and dissolution during the Neogene. The carbonate content in oceanic sediments is dependent on a complex interplay of different factors: carbonate bioproductivity, carbonate removal by chemical dissolution and mechanical erosion, terrigenous and siliceous microfossils dilution, and carbonate addition by mass or current transport. Therefore, the percent carbonate is not always sufficient to investigate the history of carbonate deposition in a specific area.

The biostratigraphic and taphonomic study of the microfossil assemblages is complemental and critical for this purpose. Specifically, biostratigraphic discontinuities, biostratigraphic zonal inversions, reworking levels, and sharp contrasts in preservation with the in-situ fossils can clarify the role played by redeposition and erosion in producing the carbonate stratigraphic record. Furthermore, the preservational state, and especially the dissolution facies, of indigenous calcareous microfossils is potentially very useful in monitoring the chemical state of bottom water (Thunell, 1976).

The problems inherent to dissolution and overgrowth in calcareous nannofossils are numerous and complex (MacKenzie and Wise, 1983), and the potential quality of their preservational state for monitoring the chemical state of bottom water awaits comparison with other dissolution-susceptible parameters. However, in this study, we have paid special attention to preservation features to contribute to a general topic of Leg 115.

Three methods are currently used to evaluate the preservation state of calcareous nannofossils: (1) a qualitative preservation scale; (2) the visual preservation scale proposed by Roth and Thierstein (1972), in which the degree of etching and overgrowth is evaluated; and (3) the taxonomic features of the assemblage, like species diversity and ratios among taxa with different preservation potential. We used all these methods to various degrees as explained below.

\section{Qualitative Preservation Scale}

This scale is based on a quick and qualitative assessment of the preservation state of calcareous nannofossils determined by their recognizability and diversity of taxa. This scale is routinely applied in the reports of the Deep Sea Drilling Project. Gener- 
ally, the effects on preservation caused by dissolution are not specified with this method, and its usefulness for paleoenvironmental inferences is limited. It is useful for evaluating the "quality" of a sample and, hence, the reliability of the inferred biostratigraphic interpretation.

The qualitative evaluation of the state of preservation of the calcareous nannofossils found within each sample was made with the following criteria:

$$
\begin{aligned}
& \mathrm{G} \text { (good) } \text { specimens exhibit little or no dissolution } \\
& \text { and/or overgrowth; } \\
& \mathrm{M}-\mathrm{G} \text { (moderate }= \text { specimens exhibit slight to moderate dis- } \\
& \text { to good) } \begin{array}{l}
\text { solution and/or overgrowth, and the iden- } \\
\text { tification of some species is impaired; }
\end{array} \\
& \mathrm{M} \text { (moderate) } \text { specimens exhibit moderate dissolution } \\
& \begin{array}{l}
\text { and/or overgrowth, and identification is } \\
\text { impaired at the specific level; and }
\end{array} \\
&= \text { specimens exhibit extreme dissolution } \\
& \text { and/or overgrowth. }
\end{aligned}
$$

These categories were determined on the basis of the "average" state of preservation of the calcareous nannofossils examined on the smear slides. There is considerable variation in the state of preservation of the individual specimens observed in any given sample.

\section{Etching-Overgrowth Scale of Roth and Thierstein}

The degree of etching and overgrowth has been plotted in the range charts with the categories proposed by Roth and Thierstein (1972) and modified by Roth (1983). We frequently observed slight to moderate etching and slight to moderate overgrowth in the same sample, which is indicative of secondary overgrowth on larger nannofossils at the expense of more delicate ones.

\section{Taxonomic Methods}

Species diversity is quite sensitive to the alteration of sediment assemblages by dissolution and diagenetic recrystallization, even if it cannot be used to distinguish between the two (Roth, 1983). Obviously, the species diversity of a specific sample is also a function of the ecology of the overlying surface-water mass and of organic evolution. The CBT sites underlie the same water mass, and Miocene and Pliocene sediments at these sites have undergone little burial diagenetic changes. Therefore, they are ideally suited to monitor the effects of dissolution on the nannofossil assemblages through time (i.e., to establish empirically the dissolution behavior of nannofossil taxa). The required census data to assess this point definitively have not been acquired in time for this report, but some preliminary considerations will be made on the basis of the available data.

Beside the total assemblage composition, other features of the assemblage, like ratios among taxa with different preservation behavior, can be used to monitor dissolution. Differential preservation sequences have been outlined by Bukry (1971), Adelseck et al. (1973), Schneidermann (1973, 1977), and Roth (1973). These studies show that discoasterids retain their identity better than all other genera during dissolution. Accordingly, Ramsay (1972, 1977), and Ramsay et al. (1973) suggested that the percentage of discoasterids in a given sample is an indicator of the intensity of the dissolution that the sample has undergone. Similarly, MacKenzie and Wise (1983) used the ratio of discoasterids to placoliths as a measure of dissolution.

We have evaluated the percentage of discoasterids by counting about 300 specimens in selected intervals at CBT sites. Within the same time slice, discoasterid abundance increases with increasing depth, thus confirming the previous assumptions on the utility of this index. We will use the abundance of discoas- terids at the various CBT sites in the different time intervals as the most objective index of dissolution intensity based on the calcareous nannofossils.

\section{BIOSTRATIGRAPHY}

Previous studies on the Neogene calcareous nannofossil biostratigraphy of the western Indian Ocean were summarized and reviewed by Müller (1977). Previous authors have predominantly relied upon the so-called standard zonations of Martini (1971) and Bukry $(1973,1975)$, code numbered by Okada and Bukry (1980). For the purpose of biostratigraphic classification of the sedimentary sequences recovered during Leg 115 , we will refer to both these zonal schemes. This approach will allow a better biostratigraphic resolution, within a zonal terminology familiar to most students of deep-sea sediments.

Most of the zonal boundaries of both schemes are easily applied in the equatorial Indian Ocean. In Figure 2, we report the zones recognized and the adopted definitions. In this figure, an asterisk superscript on an alphanumerical code name (e.g., NP25*) indicates that the zone is used in a revised sense.

Bukry $(1973,1975,1978,1985)$ often used two species events for a given boundary. Because the age of such zonal boundaries may vary, depending on which of the two events is used, we have adopted a single event to define each zone, with the use of the primary marker indicated by Bukry (Fig. 2).

Some events, and hence zonal boundaries, remain difficult to recognize, and in the following sections we discuss the procedures followed in identifying Martini's NN zones and Bukry's $\mathrm{CN}$ zones. To avoid repetitions in presenting zones at each site, we will comment here on the general features of the assemblages in the single zone intervals. In addition, we will comment on secondary events (reported on the right side of Fig. 2) that may prove useful for improving biostratigraphic resolution of the Miocene Epoch.

\section{Early Pleistocene and Pliocene Zones and Zonal Boundaries}

No major problems were encountered in applying Martini's and Bukry's zones in the Pliocene and early Pleistocene. All the zones of Martini (1971) were recognized, except for the boundary between Zones NN14 and NN15. Also, all the zones and subzones of Bukry $(1973,1975,1978,1985)$ were recognized, except for the boundary between the CN11a and CN11b subzones. Some comments on the zonal intervals and boundaries are reported below.

\section{CN13a/CN13b Boundary}

Bukry (1973) originally defined this boundary by the first occurrence (FO) of Gephyrocapsa caribbeanica, a species difficult to recognize in the optical microscope (see "Taxonomic Notes," this chapter). We recognized this boundary by the appearance of the morphometrically defined Gephyrocapsa oceanica s.1. (see "Taxonomic Notes," this chapter), which occurs in the same stratigraphic position indicated by Bukry for the FO of G. caribbeanica, which is after the last occurrence (LO) of Discoaster brouweri and before the LO of Calcidiscus macintyrei. It should be noted that the first appearance datum of $G$. oceanica s.1., an apparently synchronous event over wide areas, represents the best approximation of the Pliocene/Pleistocene boundary as recently defined in Italy (see below).

\section{CN12/CN13 (NN18/NN19) Boundary}

These zonal boundaries are defined by the LO of Discoaster brouweri. Because of reworking in many Leg 115 sites, the event is not easy to assess without detailed sampling and quantitative analysis. The boundary was recognized by the drop in abundance and by the end of the continuous common presence of 


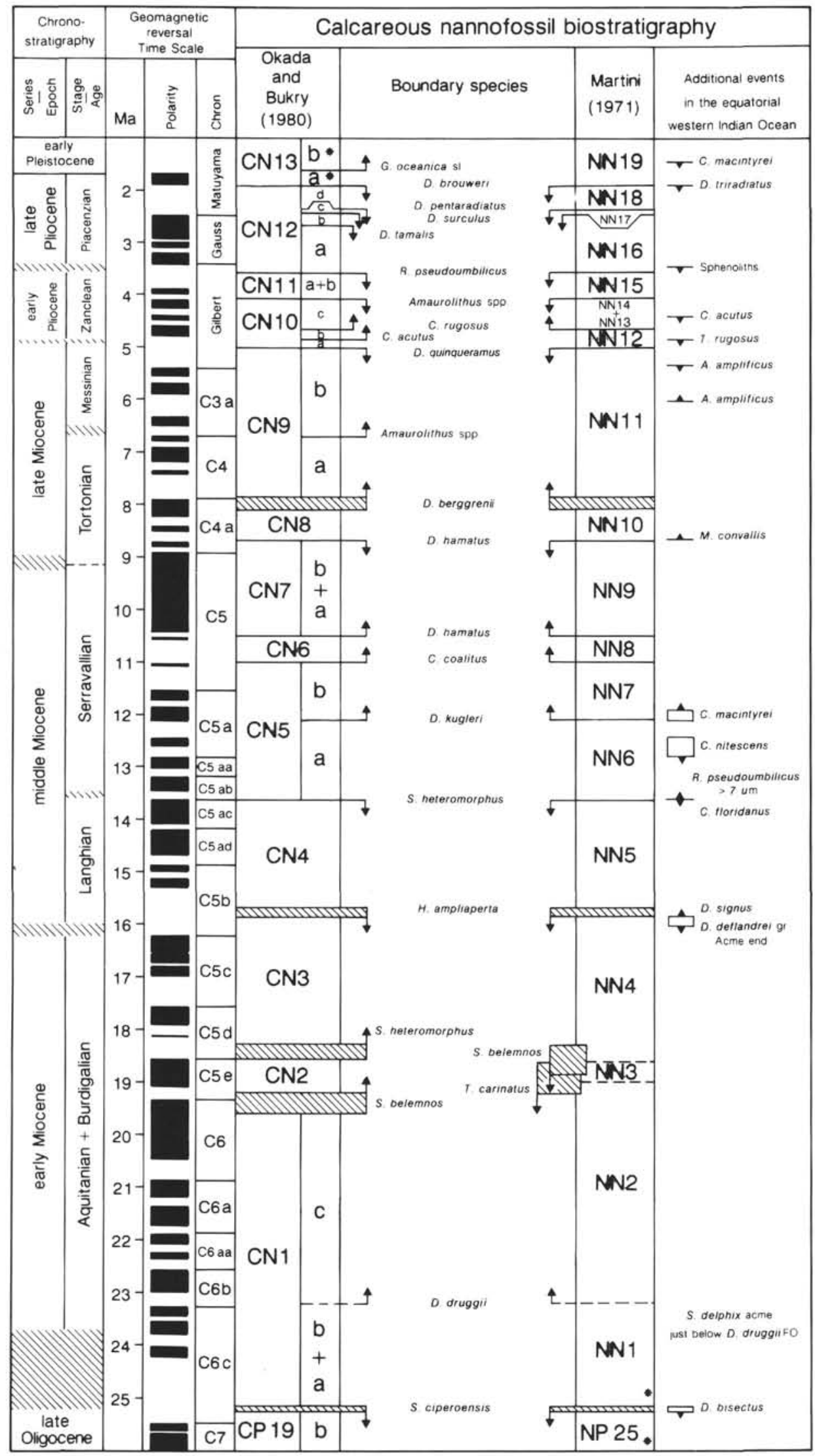

Figure 2. Adopted chronostratigraphy and calcareous nannofossil biostratigraphy and biochronology. Geomagnetic Polarity Time Scale after Berggren et al. (1985). 
the nominate species, and by the LO of Discoaster triradiatus. The latter species occurs in abundance only during the final range of $D$. brouweri and has little chance of being reworked. Similar practical criteria for recognizing these boundaries were previously followed by Rio (1982) and Parker et al. (1985).

\section{CN12b-CN12c Subzones and NN16-NN17 Zones}

The successive extinctions of Discoaster surculus and Discoaster pentaradiatus define Subzone CN12c and Zone NN17. The two events are closely spaced and often are recorded together (Backman and Pestiaux, 1986; Rio et al., 1989). In the material studied, D. surculus is generally rare in the late Pliocene, but it seems to exit the local stratigraphic record shortly before $D$. pentaradiatus.

\section{CN12a Subzone}

The top of Subzone CN12a is defined by the LO of Discoaster tamalis, which, although rare in the material studied, becomes extinct before $D$. surculus, thus allowing the recognition of Subzone CN12a. In this stratigraphic interval, members of the Discoaster variabilis group, including $D$. decorus and $D$. pansus, are recorded, in agreement with the previous findings of Bukry (1973).

\section{CN11 and NN15 Zones}

The top of Zones CN11 and NN15 is defined by the LO of Reticulofenestra pseudoumbilicus. As explained in the taxonomic notes, only the final exit of large specimens of the nominate taxon allows a consistent recognition of this boundary. The event occurs in Site 710 in the late Gilbert Chron, as it does in many other areas (Rio, 1982; Backman and Shackleton, 1983). It represents an easily recognized event in the western equatorial Indian Ocean, except at Site 716. The LO of $R$. pseudoumbilicus occurs shortly before the final exit of the last representatives of the genus Sphenolithus (S. abies and S. neoabies), which has been proposed by Bukry (1973) as a secondary event to recognize the top of Zone CN11. A similar delayed extinction of the last sphenoliths with respect to the final exit of large $R$. pseudoumbilicus was noted by Backman and Shackleton (1983) in oceanic sediments and by Rio et al. (1989) in the Mediterranean.

\section{NN14 and NN15 Zones}

The boundary between Zones NN14 and NN15 (FO of Discoaster asymmetricus) could not be differentiated in the material studied because of sporadic Pliocene occurrence of the nominate species. Furthermore, five-rayed discoasterids virtually indistinguishable from $D$. asymmetricus are common in the late Miocene. This observation is in agreement with the previous findings of Roth (1974) in the western Indian Ocean and of Bukry (1973) in other oceanic areas.

\section{CN11a/CN11b Boundary}

Boundary CN11a/CN11b is defined by the beginning of the acme of $D$. asymmetricus. Such an event cannot be recognized in the Pliocene succession of the western equatorial Indian Ocean because of the above-mentioned sporadic occurrence of the nominate species.

\section{CN10/CN11 and NN14/NN15 Boundary}

The boundary between the $\mathrm{CN} 10$ and $\mathrm{CN} 11$ Zones is defined by the LOs of Amaurolithus primus and Amaurolithus tricorniculatus; the boundary between Zones NN14 and NN15 was defined (Martini, 1971) by the LO of Ceratolithus tricorniculatus. Under the latter epithet, before the taxonomic revision of Gartner and Bukry (1975), were grouped all the nonbirefringent ceratolithids. Martini (1971) most probably intended the zone to be defined by the final exit of the last representatives of the genus
Amaurolithus. The pattern of extinction of the three normally quoted species of Amaurolithus in the early Pliocene (A. primus, $A$. delicatus, and $A$. tricorniculatus) is not well documented. Most probably the various species disappear diachronously in the different areas as suggested by the different extinction ages evaluated by various authors (see Berggren et al., 1985, and Table 2). Some discrepancy can derive from usage of different criteria when identifying these species, especially in samples in which overgrowth is a problem. In Leg 115 material, the three nominate species disappear virtually together, and this practically simultaneous extinction was used to identify the $\mathrm{CN10/CN11} \mathrm{as} \mathrm{well} \mathrm{as} \mathrm{the} \mathrm{NN14/NN15} \mathrm{boundaries.}$

\section{CN10b/CN10c (NN12/NN13) Boundary}

Boundaries $\mathrm{CN} 10 \mathrm{~b} / \mathrm{CN} 10 \mathrm{c}$ and $\mathrm{NN} 12 / \mathrm{NN} 13$ are defined by the FO of Ceratolithus rugosus. Backman and Shackleton (1983) stressed that because of intergrade forms between C. rugosus and its ancestor Ceratolithus acutus, "it is impossible to recognize a distinct first appearance of $C$. rugosus by means of qualitative methods." They suggested that, for practical purposes, the first appearance datum of $C$. rugosus can be placed at the frequency crossover of these two taxa. In this work we have drawn the datum with the first appearance of rare but typical specimens of $C$. rugosus. The event occurs in Site 710, within the Thvera Subchron, that is, in the same position detected by Backman and Shackleton (1983) in Core V28-185 from the central equatorial Pacific. Typical specimens of $C$. acutus overlap with $C$. rugosus in western equatorial Indian Ocean for a con-

Table 2. Species events defining calcareous nannofossil zonal boundaries and their assigned age estimates.

\begin{tabular}{|c|c|c|c|c|c|}
\hline Species event & $\begin{array}{l}\text { Zone } \\
\text { (top) }\end{array}$ & $\begin{array}{l}\text { Adopted } \\
\text { age }\end{array}$ & Ref. & $\begin{array}{l}\text { Berggren } \\
\text { et al. } \\
\text { (1985) }\end{array}$ & $\begin{array}{l}\text { Barron } \\
\text { et al. } \\
\text { (1985) }\end{array}$ \\
\hline C. macintyrei LO & - & $1.45 \pm 0.01$ & (2) & 1.45 & \\
\hline G. oceanica sl. FO & $\mathrm{CN} 13 \mathrm{a}$ ? & $1.58 \pm 0.3$ & (3) & 1.68 & \\
\hline D. brouweri LO & $\mathrm{NN} 18 / \mathrm{CN} 12 \mathrm{~d}$ & $1.88 \pm 0.01$ & (2) & 1.90 & \\
\hline D. pentaradiatus LO & $\mathrm{NN} 17 / \mathrm{CN} 12 \mathrm{c}$ & $2.35 \pm 0.04$ & (2) & 2.40 & \\
\hline D. surculus LO & $\mathrm{NN} 16 / \mathrm{CN} 12 \mathrm{~b}$ & $2.41 \pm 0.03$ & (2) & 2.40 & \\
\hline D. tamalis LO & $\mathrm{CN} 12 \mathrm{a}$ & $2.65 \pm 0.02$ & (2) & 2.60 & \\
\hline Sphenolithus spp. LO & CN1Ib & $3.45 \pm 0.02$ & (2) & 3.47 & \\
\hline R. pseudoumbilicus LO & $\mathrm{NN} 15 / \mathrm{CN} 11 \mathrm{~b}$ & $3.56 \pm 0.02$ & (2) & 3.50 & \\
\hline A. delicatus LO & $\mathrm{NN} 14 / \mathrm{CN} 10 \mathrm{c}$ & $4.11 \pm 0.3$ & (4) & 3.70 & \\
\hline D. asymmetricus FO & NN 13 & ND & & 4.10 & \\
\hline A. primus LO & $\mathrm{CN} 10 \mathrm{C}$ & $4.37 \pm 0.4$ & (4) & 4.40 & \\
\hline C. acutus LO & & $4.43 \pm 0.02$ & (4) & 4.57 & \\
\hline C. rugasus FO & $\mathrm{NN} 12 / \mathrm{CN} 10 \mathrm{~b}$ & $4.66 \pm 0.01$ & (4) & 4.50 & 4.7 \\
\hline C. acutus FO & CN10a & $4.85 \pm 0.03$ & (1) & 5.00 & 5.0 \\
\hline T. rugosus LO & $\mathrm{CN} 10 \mathrm{a}$ & $4.90 \pm 0.02$ & (1) & 5.00 & \\
\hline D. quinqueramus LO & $\mathrm{NN} 11 / \mathrm{CN} 9 \mathrm{~b}$ & $5.01 \pm 0.03$ & (4) & 5.60 & 5.5 \\
\hline A. amplificus LO & & $5.33 \pm 0.05$ & (4) & 5.60 & \\
\hline A. amplificus $\mathrm{FO}$ & & $6.02 \pm 0.7$ & (4) & 5.90 & \\
\hline A. primus $\mathrm{FO}$ & $\mathrm{CN} 9 \mathrm{a}$ & $6.70 \pm 0.06$ & (1) & 6.5 & 6.5 \\
\hline D. berggreni $\mathrm{FO}$ & $\mathrm{NN} 10 / \mathrm{CN} 8 \mathrm{~b}$ & $8.00 \pm 0.1$ & (4) & 8.20 & 7.3 \\
\hline D. hamatus LO & NN9/CN7b & $8.67 \pm 0.06$ & (1) & 8.85 & 8.8 \\
\hline D. hamatus FO & NN8/CN6 & 10.5 & (1) & 10.0 & 10.0 \\
\hline C. coalitus FO & NN7/CN5b & 11.1 & (1) & 10.8 & 10.8 \\
\hline D. kugleri FO & $\mathrm{NN} 6 / \mathrm{CN} 5 \mathrm{a}$ & 12.1 & (1) & 13.1 & 11.8 \\
\hline C. nitescens LO & & $12.66 \pm 0.19$ & (4) & & \\
\hline C. floridanus LO & & $13.6 \pm 0.03$ & (1) & 11.6 & \\
\hline S. heteromorphus LO & $\mathrm{NN} 5 / \mathrm{CN} 4$ & $13.6 \pm 0.03$ & (1) & 14.40 & 14.0 \\
\hline H. ampliaperta LO & $\mathrm{NN} 4 / \mathrm{CN} 3$ & $15.75 \pm 0.1$ & (4) & 16.2 & \\
\hline$D$, deflandrei acme end & & $16.05 \pm 0.5$ & (4) & & \\
\hline D. signus FO & & $16.05 \pm 0.5$ & (4) & & \\
\hline S. heteromorphus FO & $\mathrm{CN} 2$ & $18.42 \pm 0.15$ & (1) & 17.11 & 7.5 \\
\hline S. belemnos LO & NN3 & $18.55 \pm 0.25$ & (4) & 17,4 & 17.4 \\
\hline T. carinatus LO & NN2 & $18.95 \pm 0.25$ & (4) & & 18.7 \\
\hline S. belemnos FO & CNIC & $19.35 \pm 0.2$ & (4) & 21.5 & 18.2 \\
\hline D. druggii $\mathrm{FO}$ & $\mathrm{NN} 17 / \mathrm{CN} 1 \mathrm{~b}$ & 23.2 & (5) & 23.2 & 22.3 \\
\hline D. bisectus LO & & & & 23.7 & 23.4 \\
\hline S. ciperoensis LO & CP19b & $25.2 \pm 0.05$ & (1) & 25.22 & 3.7 \\
\hline
\end{tabular}

Note: $\mathrm{FO}=$ first occurrence, $\mathrm{LO}=$ last occurrence, $\mathrm{ND}=$ not detected, and Ref. $=$ refer ence, which refers to the adopted age column and represents (1) Backman et al., this volume; (2) Backman and Shackleton, 1983; (3) Rio et al., in press a; (4) this chapter; (5) Berggren et al., 1985. 
siderable interval. Most authors (see Berggren et al., 1985) do not report such an overlap.

\section{CN10a/CN10b Boundary}

Bukry (1973) defined the $\mathrm{CN10a} / \mathrm{CN} 10 \mathrm{~b}$ boundary by the FO of C. acutus and by the LO of Triquetrorhabdulus rugosus. He noted that Subzone CN1Oa is of very short duration and can well go undetected in areas of compressed sedimentation. In the material studied the two events occur virtually simultaneously. Triquetrorhabdulus rioensis, a birefringent triquetrorhabdulid recently described by Olafsson (1989), becomes extinct at the same level. It should be noted that triquetrorhabdulids are rare in their final range, but their extinction has been recorded consistently together with the FO of $C$. acutus at all sites. As mentioned below, the short CN10a Subzone represents the best approximation for the recognition of the Miocene/Pliocene boundary by means of calcareous nannofossils.

\section{Miocene Zones and Zonal Boundaries}

All the zones of Martini (1971) and Bukry (1973) of the Miocene time interval were recognized using their primary definition criteria, with the exception of the base of Zone NN1 at all sites and of the base of Zones NN5 and CN4 at the Mascarene (CBT) sites. Subzones CN8a-CN8b, CN7a-CN7b, and CN1aCN1b could not be differentiated.

\section{CN9b Subzone and the Upper NN11 Zone}

The tops of Zones CN9 and NN11 are defined by the LO of Discoaster quinqueramus, an easily detected event in the western equatorial Indian Ocean, except in shallow-water Sites 707 and 716 because of overgrowth problems. In the range charts, D. quinqueramus was grouped with Discoaster berggrenii, but typical specimens of the latter become extinct before $D$. quinqueramus, as noted by Bukry (1973) in other oceanic areas.

The base of Subzone CN9b is defined by the FO of A. pri$m u s$, another event which is easily detected in the area, although ceratoliths are generally rare. After their appearance at the base of Subzone CN9b, ceratolithids underwent considerable evolutionary pressure with the consecutive appearances, within this zonal interval, of Amaurolithus delicatus (in expanded sequences shortly after the FO of Amaurolithus primus) and of Amaurolithus amplificus (shortly after the FO of $A$. delicatus; Fig. 2).

Within the upper part of Subzone CN9b, Amaurolithus amplificus becomes extinct, in agreement with the previous findings of Bergen (1984). The FO and LO of this species is useful for intersite correlation and may provide supplementary events for further divide the rather long time interval represented by Subzone CN9b. The absolute age evaluations of the FO and LO of A. amplificus as determined at Sites 710 and 711 are reported in Table 2. Amaurolithus tricorniculatus, often recorded as appearing in Subzone CN9b (i.e., Berggren et al., 1985), has a later appearance in the material studied within $\mathrm{CN} 10 \mathrm{a} / \mathrm{CN} 10 \mathrm{~b}$ (or NN12).

The discoasterids are represented by several species, with abundant $D$. surculus and $D$. pentaradiatus. Discoaster neohamatus is virtually missing. A large form of $D$. aff. brouweri, tabulated in the range charts as Discoaster sp. 2 (see "Taxonomic Notes," this chapter), seems to have a restricted stratigraphic distribution within the lower part of this zonal interval and within the upper part of the underlying Subzone CN9a.

\section{CN9a Subzone and the Lower NN11 Zone}

The base of Subzone CN9a is defined by the FO of $D$. berggrenii, whereas the base of Zone NN11 is defined by the FO of $D$. quinqueramus. Many intergrades are present between these two related taxons, and Martini (1971) apparently considered them as a single taxonomic unit. However, end members of the group appear to have different stratigraphic ranges and should probably be considered as two related species, although it is difficult to distinguish them in intervals where both occur. We consider that, in spite of these taxonomic problems, the bases of Zones $\mathrm{CN} 9$ and NN11 are practically equivalent, being normally recognized by the FO of five-pointed ray discoasterids with a stellar knob in the central area.

The development of the knob in the central area in the $D$. berggrenii-quinqueramus lineage was gradual, and intergrades with $D$. bellus type discoasterids (tabulated as Discoaster sp. 1 in the range charts) are common. The picking of the event remains, therefore, somewhat subjective. We have considered as belonging to the $D$. berggrenii-D.quinqueramus complex forms with a distinct central area even with a poorly developed central knob. Most probably this is the concept used by most authors as demonstrated by the similar age we obtained for this event in Sites 710 and 711 with what is established in the literature (see Table 2).

A significant turnover in the calcareous nannofossil assemblage occurs within CN9a. Minylitha convallis disappears in the upper part of the interval, and Discoaster neohamatus, dominant in the underlying intervals, is strongly reduced in abundance. Typical $D$. bellus and $D$. prepentaradiatus disappear, and a concomitant increase in abundance of $D$. berggrenii and intergrade forms between $D$. bellus and $D$. berggrenii (Discoaster sp. 1 in the range charts) occurs. Discoaster pentaradiatus is present in low abundance. Typical $D$. surculus is present continuously from the base of the zone, albeit in low abundance.

In this interval we have also noted the sporadic occurrence of Discoaster loeblichii, the LO of which is used by Bukry to define the top of Zone CN8. Similar co-occurrences of D. loeblichii and $D$. berggrenii were previously noted by Proto-Decima et al. (1978), Mazzei et al. (1979), and Parker et al. (1985) in the Atlantic Ocean.

\section{CN8 and NN10 Zones}

The top of this interval was discussed above. The base is defined by the LO of Discoaster hamatus. The event can be consistently recognized in Leg 115 material; however, the form is rare close to its extinction. We have found that the event is closely approximated by the FO of $M$. convallis, an event which is more confidently determined in the area.

Bukry (1973) proposed to subdivide this biostratigraphic interval into a lower Discoaster bellus Subzone (CN8a) and an upper $D$. neorectus Subzone (CN8b) by the FO of D. neorectus and/or the FO of D. loeblichii. Roth (1974) did not find the nominate species in any of the sections he studied from Deep Sea Drilling Project (DSDP) Leg 24 in the northwestern Indian Ocean. We noted only a few specimens of typical $D$. neorectus in occasional samples, and recorded $D$. loeblichii as sporadic only at higher stratigraphic levels. Therefore, the subdivision proposed by Bukry for Zone CN9 was not adopted here.

Discoasterid assemblages are dominated by $D$. brouweri and $D$. neohamatus; $D$. prepentaradiatus is abundant in the lower part of the zone, and D. calcaris is rare or missing. Catinaster coalitus disappears at the base of this zone as does (at a slightly higher level) Catinaster calyculus.

\section{The Reticulofenestra pseudoumbilicus Paracme}

Large specimens of $R$. pseudoumbilicus enter the stratigraphic record in the middle Miocene close to the top of Zone NN5 (CN4). However, we detected an almost total disappearance of $R$. pseudoumbilicus from approximately the base of Zone NN10 (CN8) to approximately the appearance level of $A$. amplificus within lower Subzone CN9b. This long absence interval 
(paracme) represents a regional stratigraphic feature, reflecting oceanographic-climatic instability, which is not understood for the time being.

\section{CN7 and NN9 Zones}

This zonal interval, normally poorly represented in the sequences studied except at Maldives Site 714, is defined by the total range of Discoaster hamatus. For criteria used in distinguishing this species see the "Taxonomic Notes," this chapter. The FO of $D$. hamatus is virtually coincident with the appearance of other five-rayed discoasterids with more or less straight, tapering arms (Discoaster bellus), which is useful in monitoring the base of the zone as $D$. hamatus is rare.

Bukry (1973) subdivided this zone on the basis of the first evolutionary appearance of $C$. calyculus from $C$. coalitus. This subdivision was questioned by Perch-Nielsen (1985) because Thierstein (1974) and Salis (1984) found that C. calyculus appears before $D$. hamatus in the Indian Ocean and the South Atlantic, respectively. Parker et al. (1985) found the FO of $C$. calyculus together with the FO of $D$. hamatus in the North Atlantic Ocean. In the material investigated, we found rare and scattered, but typical $C$. calyculus below the FO of D. hamatus, and therefore we have not subdivided Zone CN7. One should note that in some sites the abundance of $C$. calyculus increases in abundance within the range of $D$. hamatus and this event may probably be useful. Discoaster bollii is consistently present, whereas $D$. neohamatus and $D$. prepentaradiatus appear in the lower part of this subzonal interval.

\section{CN6 and NN8 Zones}

This interval, defined by the FO of $C$. coalitus (the base) and by the FO of D. hamatus (the top) is very short (Fig. 2 and Table 2 ). It was not identified by Roth (1974) in the northwest Indian Ocean sediments retrieved during DSDP Leg 24 . We have definitively recognized it at Site 714 . In the other sites, this zone is poorly represented because of the widespread dissolution and sedimentary disturbances, but $C$. coalitus is well represented.

Discoaster brouweri and D. calcaris are common in this interval; $D$. pseudovariabilis is rare, whereas $D$. exilis seems to disappear within this zone. According to Ellis (1981) and PerchNielsen (1985), Discoaster kugleri disappears near the base of this zone. In the material investigated, D. kugleri is generally rare and has not been observed in this zone.

\section{CN5b Subzone and NN7 Zone}

The FO of Discoaster kugleri defines the base of these zones. This event is considered as poor by many authors (e.g. Gartner and Chow, 1985), and alternative criteria have been proposed to recognize this boundary. Bukry (1973) proposed the LO of $C y$ clicargolithus floridanus as a secondary marker for the base of CN5b Subzone. Ellis (1981) suggested as a substitute event the FO of Discoaster bollii. Gartner and Chow (1985) suggested that the LO of Coronocyclas nitescens may be useful in subdividing the interval above the LO of Sphenolithus heteromorphus and below the FO of $C$. coalitus.

In our material, D. kugleri is common and easily recognized in some samples, but it is lacking in many intervening samples. Its recognition in overgrown material is difficult. Site 714 contains the most complete sequence over the interval of interest, and we noticed that the LO of $C$. nitescens is very close to the level we consider to be the appearance level of $D$. kugleri. The LO of $C$. floridanus occurs at much lower stratigraphic levels, slightly after the LO of $S$. heteromorphus.

A similar short-spaced extinction between $S$. heteromorphus and $C$. floridanus was observed by Olafsson (1989) in the equatorial Pacific and the equatorial Atlantic. Parker et al. (1985) found that the LO of $C$. floridanus occurs well below the FO of
D. kugleri in the mid-latitude North Atlantic DSDP Site 563. We consider that the $C$. floridanus LO is a useful event, but its use in recognizing the base of Subzone CN5b or the base of Zone NN7 may lead to conflicting chronological correlations among different areas.

In this biostratigraphic interval, forms of the $D$. variabilis group are the dominant discoasterids. Discoaster subsurculus, $D$. moorei, and $D$. exilis are well represented, whereas $D$. bollii occurs rarely and discontinuously. Among placoliths, $R$. pseudoumbilicus is dominant, and large-sized Calcidiscus (C. macintyrei) appears within this interval.

\section{CN4/CN5 (NN5/NN6) Boundary}

The NN5/NN6 and CN4/CN5 boundaries are defined by the LO of $S$. heteromorphus, which is easily recognized in the material studied. Close to this zonal boundary, a major turnover in the Miocene nannofossil assemblages is recorded. With special reference to Maldives Site 714 , which provides the most complete sequence across this interval, the following major changes were recorded:

1. Large $R$. pseudoumbilicus ( $>7 \mu \mathrm{m}$ ) appears at the NN5/ NN6 boundary, and becomes the dominant element among the medium-sized placoliths. Within the lower part of Zone NN6, reticulofenestrids increase gradually to a maximum size larger than $10 \mu \mathrm{m}$.

2. Cyclicargolithus floridanus, the dominant element in the placolith assemblages in the underlying intervals, disappears in the area shortly after the LO of S. heteromorphus.

3. Among the sphenoliths, small forms related to Sphenolithus abies become abundant, and the spined sphenoliths, which were the dominant forms in the underlying intervals, become definitively extinct.

4. The discoasterid assemblage is dominated by slender-arm forms, often of large size (e.g., D. exilis, D. variabilis, $D$. aff. calcaris, and $D$. brouweri). The short-armed discoasterids of the Discoaster deflandrei group have practically vanished.

5. Triquetrorhabdulus rugosus and Triquetrorhabdulus rioensis enter the stratigraphic record approximately at the NN5/ NN6 boundary.

6. In all of the investigated sites, a special feature is represented by a bloom of small- and medium-sized Dictyococcites in the uppermost part of Zone NN5. This group is a minor component of the assemblage in the underlying intervals, where it is absent or represents only a few percent of the placoliths. Rather suddenly in the upper part of the zone, and for a short interval, it makes up to $30 \%-60 \%$ of the total assemblage. Dictyococcites productus, Dictyococcites hesslandi, and Dictyococcites antarcticus were determined in this interval. The occurrence of specimens virtually indistinguishable from $D$. antarcticus in low-latitude sediments is significant because this species is considered as restricted to the high latitudes (Haq, 1980).

\section{CN3/CN4 (NN4/NN5) Boundary}

The LO of Helicosphaera ampliaperta LO is the primary criterion of definition of NN4/NN5 and $\mathrm{CN} 3 / \mathrm{CN} 4$ boundaries. However, in many open-ocean sediments, the nominate species is very rare or absent, and the two zones cannot be distinguished. It is not clear if $H$. ampliaperta is missing from most openocean sections because of its low resistance to dissolution or if it is regionally and/or ecologically restricted.

Various criteria have been used by different workers to separate the nominate zones when $H$. ampliaperta is not present:

1. Bukry (1973) proposed the end of the $D$. deflandrei acme as an alternative event in low-latitude sediments, without defining it quantitatively. Parker et al. (1985) noted that the use of an 
acme to define a zonal boundary is not generally desirable, because such an event may vary from place to place for a variety of reasons.

2. Jiang and Gartner (1984) in the Walvis Ridge and Parker et al. (1985) in the mid-latitude North Atlantic used the first appearance of long-armed discoasterids (D. variabilis group) to distinguish Zones $\mathrm{CN} 3$ and CN4.

3. Roth (1974) drew the NN4/NN5 boundary at the level where there is a shift in dominance among discoasterids from the $D$. deflandrei group to long-armed discoasters like $D$. exilis and $D$. variabilis. Parker et al. (1985) noted that this change may occur at a different level from the two previous ones.

4. Müller (1974) drew the NN4/NN5 boundary at the FO of D. exilis, following Martini and Worsley (1971). Bukry (pers. comm. in Ellis, 1981) considered this event as difficult to assess because overgrowth on discoasterids makes the distinction between $D$. exilis and $D$. variabilis difficult.

5. Bukry (1978), who considered most of the above-mentioned events variable in the different areas because of ecological and preservational vagaries, proposed (following Hekel, 1973) the FO of Calcidiscus macintyrei as a convenient means of identifying the $\mathrm{CN} 3 / \mathrm{CN} 4$ boundary. He specified that $C$. macintyrei is "a large species with a distinct central tube."

In our material $H$. ampliaperta is present only in Maldives Sites 714 and 715 . We tried to determine the stratigraphic positions of the alternative criteria mentioned above vs. the LO of $H$. ampliaperta in the Maldives sites. Quantitative analyses of the discoasterid assemblages indicate that the $D$. deflandrei group drops in abundance in a significant manner within the interval. If we assume as the definition of the acme end of the nominate species its drop from values above $30 \%$ to values below that same amount, the event occurs just before the LO of $H$. ampliaperta in the Maldives sites and is quite distinct. This event coincides with the shift in dominance between short-armed and long-armed discoasters, which can be determined also in overgrown assemblages (i.e., at Site 707). The acme end of $D$. deflandrei is coincident with the FO of the Discoaster signus- $D$. tuberi group. Such a coincidence was also detected at all the Mascarene (CBT) sites.

Based on these results, we have used the drop in abundance below $30 \%$ of $D$. deflandrei and the concomitant appearance of the $D$. tuberi-D. signus group to distinguish the NN4 (CN3) and NN5 (CN4) Zones in sites where $H$. ampliaperta is missing.

\section{CN2/CN3 and NN3/NN4 Boundary}

Martini defined the base of Zone NN4 by the LO of $S$. belemnos, whereas Bukry defined the base of $\mathrm{CN} 3$ by the FO of $S$. heteromorphus. The relative ranges of these two species are debated. They are reported as co-occurring at DSDP Site 140 in the Atlantic Ocean by Bukry (1972), at DSDP Site 610 in the North Atlantic Ocean by Takayama and Sato (1985) and in the equatorial Pacific by Pujos (1985). Olafsson (1989) found that at DSDP Site 608 in the North Atlantic Ocean the two species do not overlap. In our material the two events are virtually coincident, although sedimentation appears compressed or affected by gaps at most sites in the corresponding interval.

\section{NN3 Zone}

Zone NN3 is defined as the interval from the LO of Triquetrorhabdulus carinatus (base) to the LO of Sphenolithus belemnos (top). However, the range of $T$. carinatus appears to be paleogeographically controlled. It is recorded often as becoming extinct, or very rare, below the LO of $S$. belemnos (e.g., Parker et al., 1985; Olafsson, 1989). In the western equatorial Indian Ocean, $T$. carinatus is abundant in the early Miocene, and at Maldives Site 715 it becomes extinct before the $\mathrm{LO}$ of $S$. belem- nos. This finding would indicate that Zone NN 3 is present in the area. Because the two events occur virtually simultaneously in all the other sites, we infer a generalized hiatus. The presence of a hiatus in this interval is in agreement with an observed strong increase in dissolution of calcareous nannofossils in the upper part of Zone NN2 (Subzone CN1c) and the basal part of Zone NN4 $(\mathrm{CN} 3)$ and also with the observation that Zone CN2 is virtually missing at the CBT sites.

\section{CN2 Zone}

Zone $\mathrm{CN} 2$ is defined as the interval between the FOs of $S$. belemnos and $S$. heteromorphus. This interval is only present at Maldives Sites 714 and 715, where, nevertheless, it is very short. At all CBT sites in the northern Mascarene area, this zonal interval is virtually absent and a hiatus is inferred (see the comments on Zone NN3).

\section{CN1b/CN1c (NN1/NN2) Boundary}

Martini (1971) defined the NN1/NN2 boundary by the FO of Discoaster druggii; Bukry (1978) noted that the range of this species is uneven and suspected some major ecological control in its distribution; he, therefore, retained Zone NN2 at the subzonal rank $(\mathrm{CN} 1 \mathrm{c})$. Many authors have noted that it may be difficult to recognize $D$. druggii in overgrown material (e.g., Olafsson, 1989). Müller (1977) states that the species is rare in the Indian Ocean. In the material studied $D$. druggii is sporadic.

Olafsson (1989) used the FO of Triquetrorhabdulus serratus in Site 667 as an alternative event in the eastern equatorial Atlantic. In our material, Triquetrorhabdulus serratus is present and easily recognized, as it is present only from the lower part of Zone NN2 (Subzone CN1c). However, it occurs in low abundance, and correlations among different sites are inconsistent.

An interesting feature that has been useful in confirming the consistency of the determined FO of $D$. druggii is the presence of a correlatable, easily detected, short acme interval of Sphenolithus delphix, slightly below the FO of $D$. druggii at all the sites investigated. Sphenolithus capricornutus is recorded with $S$. delphix in this interval; the two species are virtually restricted to the upper part of NN1 (Subzone CN1c) in the area.

\section{CN1a/CN1b Boundary}

The boundary between these subzonal intervals, generally correlated to the lower part of Zone NN1, is defined by the acme end of Cyclicargolithus abisectus. The first problem regarding the recognition of this boundary is that Bukry did not define the acme end quantitatively. A second problem is the poor taxonomic state of the nominate species. Cyclicargolithus abisectus was originally described by Müller (1970) from advanced early Miocene (NN3 Zone) sediments as circular to weakly elliptical placoliths ranging in size from 8.5 to $11 \mu \mathrm{m}$.

Bukry and Percival (1971) placed this species in the genus Dictyococcites Black, referring to it as a circular to subcircular placolith ranging in size from 12 to $16 \mu \mathrm{m}$. The species, according to Müller (1977), ranges in the lowermost middle Miocene (NN5-NN6 Zones). Cyclicargolithus abisectus, as defined by Müller (1970), can be difficult to recognize from C. floridanus, especially if the material is not well preserved.

In this work we have distinguished large forms of $C$. abisectus (larger than $10 \mu \mathrm{m}$ ) from smaller forms (C. abisectus s.1. in the range charts). The large forms become extinct very close to the LO of Sphenolithus ciperoensis (the definition of the base Zone $\mathrm{CN} 1$ ), and no acme was recognized that used the taxonomic concept of $C$. abisectus. Medium-sized $C$. abisectus are distributed as high up as the lower part of Zone NN4, with no increase in abundance evident in the interval between the LO of $S$. ciperoensis and the FO of D. druggii. Therefore, Subzones $\mathrm{CN} 1 \mathrm{a}$ and $\mathrm{CN} 1 \mathrm{~b}$ of Bukry could not be differentiated. 


\section{CP19/CN1 and NP25/NN1 Boundary}

The recognition of these zonal boundaries, which are generally correlated and are critical for the recognition of the Oligocene/Miocene boundary (see discussion below), is not always consistent among different authors. At least three criteria are used to recognize them, which may not be equivalent:

1. the LO of Helicosphaera recta, the original definition of the NP25/NN1 boundary;

2. the LO of Sphenolithus ciperoensis, proposed by Bukry as the primary criterion for defining the CP19/CN1 boundary; and

3. the LO of Dictyococcites bisectus, proposed by Bukry as a secondary criterion for recognizing the CP19/CN1 boundary and used by many authors to approximate the NP25/NN1 boundary when helicoliths are missing (e.g., Berggren et al., 1985).

Helicosphaera recta rarely occur in oceanic sediments; $S$. ciperoensis is most common in low-latitude sediments; whereas $D$. bisectus is a more cosmopolitan form, although it may be entirely missing in some low-latitude sediments (Ruddiman, Sarnthein, et al., 1988). As a result, the relative final range of the three species is not well established, and simultaneous (Bizon and Müller, 1979) as well as reversed extinctions are reported in the literature (Berggren et al., 1985).

Helicosphaera recta is missing at all Leg 115 sites except in Site 714. Dictyococcites bisectus is present, but it becomes rare in its final range. Sphenolithus ciperoensis is always abundant. At Site 714 , the three species disappear almost simultaneously. However, because $H$. recta and $D$. bisectus are rare, the established relationships may be of only local value. A further complication arises in that genuine $H$. recta has also been detected in spot samples also in the advanced early Miocene (Zone NN4) at Sites 714 and 715 .

Sphenolithus ciperoensis is abundant and continuously present in all the investigated sections, providing a reliable biostratigraphic signal, and its extinction was used to recognize the boundary between NP25 and NN1.

\section{BIOCHRONOLOGY}

One of the most remarkable advances in stratigraphy in recent years has been the correlation of planktonic biostratigraphy with the Geomagnetic Polarity Time Scale (GPTS). Direct correlation of calcareous nannofossil events with the GPTS were established in several different areas for the Pleistocene and the Pliocene (Backman and Shackleton, 1983; Berggren et al., 1985), and the calcareous nannofossil biochronology of this interval can be considered well established. On the contrary, the Miocene calcareous nannofossil biochronology is in a flux state because only a few sequences with good magnetostratigraphy are available (Berggren et al., 1985).

During Leg 115 magnetic stratigraphy was obtained at Sites 710 and 711 . Although the stratigraphic records at these sites are not uncomplicated (see "Site Summaries," this chapter), they are important equatorial records for calcareous nannofossil biochronology.

The results obtained are basically in agreement with or indicate only minor revisions to the generally accepted late Miocene and Pliocene calcareous nannofossil biochronology summarized by Berggren et al. (1985). On the contrary, they indicate that major revisions are needed in the middle Miocene (Backman et al., this volume). The biochronology adopted in this work (Fig. 2 and Table 2) is largely taken from Berggren et al. (1985) for the Pliocene and late Miocene, from Backman et al. (this volume) for the middle Miocene, and from interpolated ages obtained in this work in the early Miocene, as commented upon elsewhere.

\section{CHRONOSTRATIGRAPHY}

Precise placement of chronostratigraphic boundaries is of interest because chronostratigraphy represents the best available basis for international communication and correlation. The internationally adopted chronostratigraphic units of the Pliocene and the Miocene are designated in European sequences, which are often poorly constrained in the plankton biostratigraphic and magnetostratigraphic scales. Therefore, their recognition outside the type regions is often difficult. In the present work, we have not used the European stage-age units as a chronostratigraphic frame, and we will make reference to the informal subdivision of the Miocene and Pliocene in subseries-subsystems. Even this large-frame subdivision is, however, not devoid of problems, and procedures followed and reported in Figure 2 need to be clarified.

\section{The Pliocene/Pleistocene Boundary}

The age and recognition of the Pliocene/Pleistocene boundary has long been, and continues to be, a controversial issue. This is due in large part to different philosophical approaches in defining the boundary (see Berggren and Van Couvering, 1974, and Pelosio et al., 1980, for reviews). Beginning with Forbes (1846), many workers have chosen to define the Pliocene/Pleistocene boundary on the basis of climatic criteria, equating it with the buildup of Northern Hemisphere glaciation. Recent studies clearly indicate that this important climatic event occurred at about 2.5 Ma (Shackleton et al., 1984), and this event can be recognized within the sequence, Pliocene by definition, of the Arda Valley in northern Italy (Rio et al., 1988).

In contrast, the other approach to defining the Pliocene/ Pleistocene boundary is based on stratigraphic principles and recognizes the need of establishing a boundary stratotype. The boundary is a unique time horizon and should therefore represent a universal standard of reference for dating purposes. In this context, the climatic significance is not the driving criterion for definition and recognition of the boundary.

The problem of establishing an acceptable definition of the Pliocene/Pleistocene boundary appears to have been resolved finally with the acceptance of the Vrica section (Calabria, southern Italy) as the stratotype for the boundary (Aguirre and $\mathrm{Pa}-$ sini, 1985). The top of the laminated level "e" in the Vrica section was selected as the marker horizon for the boundary (Aguirre and Pasini, 1985). This level is just above the top of the Olduvai Subchron, yielding an age of approximately $1.6 \mathrm{Ma}$ for the Pliocene/Pleistocene boundary (Tauxe et al., 1983a).

In the present work, we have recognized the boundary by the FO of Gephyrocapsa oceanica, which occurs in the equatorial Indian Ocean just above the top of the Olduvai Subchron, as it does at the boundary stratotype section in the Mediterranean.

\section{Pliocene Chronostratigraphy}

There is a fairly large consensus in subdividing the Pliocene series into two intervals (early and late Pliocene), for which the boundary is normally approximated by means of calcareous nannofossils by the LO of Reticulofenestra pseudoumbilicus, close to the upper part of the Gilbert Chron. This procedure, which was followed here, is in agreement with the fact that the basal Piacenzian (late Pliocene) stratotype has an estimated age of about 3.4-3.6 Ma (Rio et al., 1988).

\section{Miocene/Pliocene Boundary}

The Miocene/Pliocene boundary has traditionally been considered to be coincident with the restoration of open-marine conditions in the Mediterranean following the Messinian "sa- 
linity crisis." Accordingly, Cita (1975) proposed the boundary stratotype for the Miocene/Pliocene boundary in the Capo Rossello section (southern Sicily) at the sharp contact between the brackish water "Arenazzolo formation" and the open-marine "Trubi formation." Correlation of this boundary to the openocean record was difficult because of the lack of a magnetostratigraphy in the type section and because of the fact that the late Miocene sediments at Capo Rossello are nonmarine.

Not surprisingly, the recognition of this boundary was and still is controversial, and one may derive a different identification of this boundary in the same sequence with magnetostratigraphy, planktonic foraminifers or calcareous nannofossils (i.e., Parker et al., 1985).

According to Berggren et al. (1985), the age of the Miocene/ Pliocene boundary is approximately $5.4 \mathrm{Ma}$. However, the magnetostratigraphic results from Leg 107 (Channell et al., 1989), as well as new results from land sections in Calabria (Zijderveld et al., 1986; Channell et al., 1988), indicate that the boundary occurs slightly below the Thvera Subchron, within the upper part of the lowest reversed interval of the Gilbert Chron. Ages of 4.83 and $4.93 \mathrm{Ma}$ were estimated for the boundary by Zijderveld et al. (1986) and Channell et al. (1988), respectively.

The nannofossil criterion generally used by nannofossil paleontologists to pick this boundary is the LO of Discoaster quinqueramus (e.g., Gartner et al., 1987; Parker et al., 1985). Martini (1971) and Bukry (1973), however, placed this event below the boundary. Bukry tentatively suggested the LO of Triquetrorhabdulus rugosus and the FO of Ceratolithus acutus (top of Subzone $\mathrm{CN10a}$ ) as the best approximation of the Miocene/ Pliocene boundary on the basis of correlations with Mediterranean stratotypes. The calcareous nannofossil biochronology adopted in the present work (Table 2) indicate that all these events can be used to approximate the boundary, but the top of Subzone CN10a (about $4.85 \mathrm{Ma}$ ) seems to be the most precise criterion for recognizing the Miocene/Pliocene boundary in the equatorial Indian Ocean. Therefore, we adopted it here.

\section{Middle/Late Miocene Boundary}

The middle/late Miocene boundary is generally considered to be defined by the base of the Tortonian stage (Berggren et al., 1985). However, the published data on the Tortonian stratotype in the Rio Mazzapiedi section (northern Italy) do not make it possible to determine unequivocally the bio- or magnetostratigraphic position of the Serravallian/Tortonian boundary and, hence, of the middle/late Miocene boundary. The base of the stratotype section was assigned by Martini $(1971,1975)$ to Zone NN9 based on the occurrence of Discoaster hamatus in Sample 4, which was interpreted by Martini (1975) as the LO of this taxon. This is the same level as the supposed FO of Neogloboquadrina acostaensis (Cita et al., 1965). Berggren et al. (1985) noted that the lower part of the Rio Mazzapiedi section (below Sample 4) contained poor and nondiagnostic microfossils; therefore, the occurrences of $N$. acostaensis and D. hamatus may not, in fact, be actual initial and final stratigraphic occurrences.

Based on magnetochronologic considerations and on the fact that the FO of $N$. acostaensis occurs in DSDP Site 563 within Zone NN8, Berggren et al. (1985) placed the Serravallian/Tortonian boundary within Zone NN8, ignoring the fact that $D$. hamatus, present in the basal-most Tortonian, has not yet appeared in NN8 time interval. Recent work in italian sections (E. Fornaciari, D. Rio, and R. Sprovieri, unpubl. data) indicate that the FO of $N$. acostaensis occurs in the Mediterranean before the FO of $D$. hamatus, as reported in many oceanic areas. This finding suggests that most probably the occurrence of $N$. acostaensis in the basal Tortonian (together with the last $D$. hamatus) in the section does not represent the FO of this taxon, and that the base of the Tortonian lies most probably in the up- per part of Zone NN9. We have drawn the boundary between the middle and the late Miocene in the upper part of this zone, in agreement with the procedure followed previously by Barron et al. (1985) and by Haq et al. (1987).

\section{Early/Middle Miocene Boundary}

The early/middle Miocene boundary is equivalent to the base of the Langhian stage (Berggren et al., 1985). This boundary is linked biostratigraphically to the FO of Praeorbulina glomerosa curva (Cita and Premoli Silva, 1968), which in the stratotype section occurs above the FO of Sphenolithus heteromorphus and just below the LO of Helicosphaera ampliaperta, within the upper part of Zone NN4 (Martini, 1971). There is general agreement among calcareous nannofossil paleontologists to draw the early/middle Miocene within the upper or at the top of Zone $\mathrm{NN} 4$, and we have followed this practice. It should be noted that this zonal boundary (see "Biostratigraphy" section, this chapter) was recognized at all sites but the Maldives by means of the acme end of $D$. deflandrei, as $H$. ampliaperta is missing.

\section{Oligocene/Miocene Boundary}

The Oligocene/Miocene boundary is one of the most controversial boundaries in the stratigraphic column. Conflicts derive basically from the limits of the European late Oligocene (Chattian) and early Miocene (Aquitanian) stratotypes, which are located quite apart one from the other (in Germany and in southwest France, respectively). These stratotypes are poor in planktonic fossils and represent short intervals of time bounded by unconformities.

No consensus has been reached so far in proposing a boundary stratotype section for the Oligocene/Miocene boundary, and different specialists use quite different criteria to recognize it. Recent extensive reviews of this problem were performed by Srinivasan and Kennett (1983) and Berggren et al. (1985).

Calcareous nannofossil paleontologists have used quite different criteria to recognize the Oligocene/Miocene boundary. Martini (1971) drew the boundary at the top of Zone NP25. Bukry (1973) and Roth (1974) put the boundary at the top of Subzone CN1a, at the acme end of $C$. abisectus. Gartner (1974) used the FO of Triquetrorhabdulus carinatus to recognize the boundary in the northern Indian Ocean. In recent years, most nannofossil paleontologists have recognized the Oligocene/Miocene boundary at the top of Zone NP25 or of Zone CP19. However, we have discussed above the fact that the tops of these zones are recognized by different events (LOs of $\mathrm{H}$. recta, S. ciperoensis, and $D$. bisectus) which may have different geochronologic meanings in different areas. In the western equatorial Indian Ocean, the three events occur virtually together at Site 714 , and the Oligocene/ Miocene boundary was picked by using the LO of $S$. ciperoensis, which is an easily recognized event in all the considered sequences.

Planktonic foraminiferal criteria widely used in the last years to pick the boundary are the FO of Globorotalia kugleri (Berggren et al., 1985) and the FO of Globoquadrina dehiscens (Srinivasan and Kennett, 1983). Depending upon the chosen biostratigraphic event (LOs of $S$. ciperoensis, D. bisectus, and $H$. recta and FOs of $G$. kugleri or $G$. dehiscens), different positions of the Oligocene/Miocene boundary will result in the same section.

\section{SITE SUMMARIES}

Preliminary discussions on the biostratigraphic sequences at each site of Leg 115 are included in the appropriate site reports in Backman, Duncan, et al. (1988). We have already summarized the general problems and features of the calcareous nannofossil biostratigraphy of the western equatorial Indian Ocean. Therefore, we will keep our verbal description to a minimum 
here and will concentrate instead on problems and details encountered in completing this study. We will briefly summarize the stratigraphy at each site, with special emphasis on preservation features, redeposition processes, and hiatuses. Some comments on biochronologic aspects not discussed previously will be made.

Fundamental data are reported in Tables 3 through 30 and are synthesized in columnar logs reported in Figures 3, 5, 8, 12, 14 , and 19-21.

\section{South Mascarene Plateau Sites (Sites 705 and 706)}

Sites 705 and 706 are located $3 \mathrm{nmi}$ from each other on the eastern shoulder of the Mascarene Plateau along the northern margin of the Nazareth Bank (Fig. 1) at a moderately deep location (Table 1). The sediments retrieved at Site 705 are coarsegrained foraminiferal ooze, late Pliocene-Pleistocene in age.

The late Pliocene-early Pleistocene calcareous nannofossil distribution at Site 705 is reported in Tables 3 and 4.

Nannofossils are common, the preservation state is moderate, and reworking is low. All the late Pliocene zonal intervals were recognized (Table 3). Okada (this volume) presents the late Pleistocene and early Oligocene nannofossil biostratigraphy from Site 706.

\section{The Carbonate Bathymetric Transect (Sites 707 through 711)}

Sites 707 through 711 (Table 1) represent a Neogene Indian Ocean carbonate core transect (see "Introduction" section, this chapter).

Our objectives in studying the sequences retrieved at these sites were (1) to establish a chronologic framework within which to study the time-dependent variability of carbonate production and dissolution, and (2) to contribute to the understanding of the factors that actually control carbonate sedimentation in the area considered. We will show that the Neogene stratigraphic records of CBT sites are the result of a complex interplay of the various factors, previously discussed, which control carbonate accumulation in the deep sea. Erosion at the shallow-water sites, dissolution, and redeposition in the deep-water sites occurred. The results shown below indicate the need for detailed paleontologic studies before any attempt at modeling the carbonate system budget can be made.
In this section, we will summarize the stratigraphic information at each site in order of decreasing water depth (Figs. 3-14), and then we will discuss the implications of the results obtained by correlating the stratigraphic records of the CBT sites during different time intervals (Figs. 15-17).

\section{Site 707}

Site 707 is located on the northwestern part of the Mascarene Plateau, between the Seychelles Bank and Saya de Malha, 60 nmi from DSDP Site 237 (Fig. 1). Site 707 is the shallow-water sequence of the Neogene Indian Ocean CBT. Three holes were drilled at Site 707 (Table 1), and Hole 707C reached the basement. Sedimentary sequences above the basement range from early Paleocene to Pleistocene in age.

Only the latest Oligocene through earliest Pleistocene stratigraphy of Hole 707A was considered for detailed shore-based study. The calcareous nannofossil distribution and biostratigraphy is reported in Table 5 (microfiche, back pocket) and in Figure 3. Events are summarized in Table 6.

Calcareous nannofossils are present throughout the Miocene and Pliocene sequence, ranging from common to abundant. Their abundance increases downhole. The preservation state is from moderate to poor because of the strong overgrowth of discoasterids. This made the identification of some zonal markers difficult. Etching of nannofossils is minimal, as expected at this shallow-water site. Species diversity, albeit not quantified for the moment, is the highest observed in the CBT sites. Helicoliths, scyphospherids, and pontospherids are notably more abundant and more continuously present than in the other CBT sites. Discoasterid abundance is low, never exceeding $40 \%-50 \%$ of the total assemblage (Fig. 3). Reworking is moderate.

All the adopted biozones of the late Miocene and Pliocene were identified. The early and middle Miocene interval is compressed, and is affected by major hiatuses (Fig. 3). Specifically, Zones NN4 and NN5 are compressed. Zones NN3 and NN2 are missing. We will see that sediments belonging to the latter two biostratigraphic intervals are found redeposited in deeper water Site 709.

\section{Site 709}

Site 709 is located at $3049 \mathrm{~m}$ water depth, in a small basin perched near the summit of the Madingley Rise, a regional topographic high between the Carlsberg Ridge and the Northern

Table 3. Distribution of calcareous nannofossil taxa at Hole 705A.

\begin{tabular}{|c|c|c|c|c|c|c|c|c|c|c|c|c|c|c|c|c|c|c|c|c|c|c|c|c|c|c|c|c|c|c|c|c|c|c|c|c|c|}
\hline $\mathrm{NN}$ & $\mathrm{CN}$ & $\begin{array}{l}\text { Core, section, } \\
\text { interval }(\mathrm{cm})\end{array}$ & $\begin{array}{l}\text { Depth } \\
\text { (mbsf) }\end{array}$ & 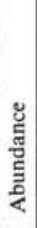 & 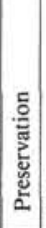 & 总 & 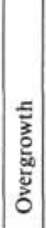 & 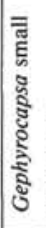 & 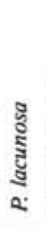 & 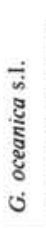 & 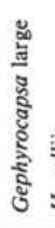 & : & 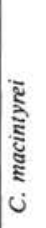 & $\begin{array}{l}0 \\
0 \\
0 \\
\frac{2}{2} \\
\frac{2}{2}\end{array}$ & $\begin{array}{l}\frac{n}{3} \\
\frac{a}{5} \\
\vdots\end{array}$ & 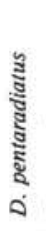 & 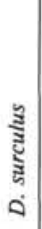 & 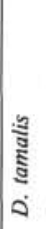 & 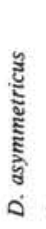 & $\begin{array}{c}\text { जै } \\
\text { है } \\
\text { है } \\
ن\end{array}$ & $\begin{array}{l}\frac{y}{5} \\
\frac{5}{2} \\
\text { อ } \\
0\end{array}$ & 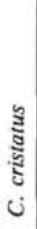 & 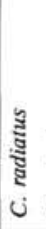 & $\frac{y}{\tilde{z}}$ & 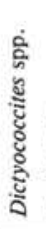 & 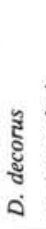 & 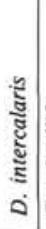 & 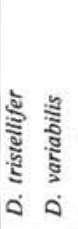 & 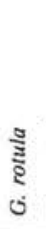 & 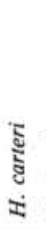 & 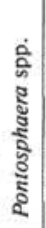 & 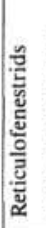 & 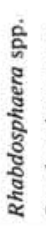 & 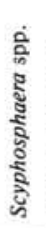 & 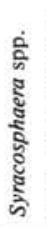 & 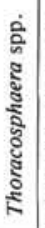 & 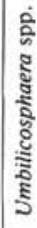 \\
\hline 19 & 13 & $\begin{array}{l}1 \mathrm{H}-5,30 \\
1 \mathrm{H}-6,41 \\
1 \mathrm{H}-\mathrm{CC} \\
2 \mathrm{H}-1,130\end{array}$ & $\begin{array}{l}8.50 \\
9.80\end{array}$ & $\begin{array}{l}\mathrm{C} \\
\mathrm{C} \\
\mathrm{C} \\
\mathrm{A}\end{array}$ & $\begin{array}{l}G \\
G \\
G \\
G\end{array}$ & $\begin{array}{l}\text { E0 } \\
\text { E0 } \\
\text { E0 } \\
\text { E0 }\end{array}$ & $\begin{array}{l}\mathrm{O} 0 \\
\mathrm{O} 0 \\
\mathrm{O} 0 \\
\mathrm{O} 0\end{array}$ & $\begin{array}{l}C \\
C \\
C \\
C\end{array}$ & $\begin{array}{l}\text { A } \\
\text { A } \\
\text { A } \\
\text { C }\end{array}$ & $\begin{array}{l}\mathrm{C} \\
\mathrm{C} \\
\mathrm{C} \\
\mathrm{F}\end{array}$ & R & $\mathrm{F}$ & C & & & $\mathbf{R}$ & & & & $\mathbf{R}$ & $\begin{array}{l}C \\
C \\
C \\
C\end{array}$ & $\begin{array}{l}\mathrm{R} \\
\mathrm{R} \\
\mathrm{R} \\
\mathrm{R}\end{array}$ & $\begin{array}{l}C \\
C \\
C \\
C\end{array}$ & $\mathbf{R}$ & & & & & $\begin{array}{l}\mathrm{R} \\
\mathrm{F} \\
\mathrm{C} \\
\mathrm{C}\end{array}$ & $\begin{array}{l}\mathrm{C} \\
\mathrm{C} \\
\mathrm{C} \\
\mathrm{C}\end{array}$ & $\begin{array}{l}\mathrm{R} \\
\mathrm{R} \\
\mathrm{R} \\
\mathrm{R}\end{array}$ & $\begin{array}{l}\mathrm{R} \\
\mathrm{R} \\
\mathrm{R} \\
\mathrm{R}\end{array}$ & $\begin{array}{l}\mathrm{F} \\
\mathrm{R} \\
\mathrm{F} \\
\mathrm{F}\end{array}$ & $\begin{array}{l}\mathrm{R} \\
\mathrm{R}\end{array}$ & $\begin{array}{l}\mathrm{C} \\
\mathrm{C} \\
\mathrm{C} \\
\mathrm{C}\end{array}$ & $\begin{array}{l}\mathrm{F} \\
\mathrm{R} \\
\mathrm{F} \\
\mathrm{F}\end{array}$ & $\begin{array}{l}\mathrm{F} \\
\mathrm{F} \\
\mathrm{C} \\
\mathrm{C}\end{array}$ \\
\hline 18 & $12 \mathrm{~d}$ & $2 \mathrm{H}-2,130$ & 11.30 & A & G & E0 & 00 & $\mathrm{~F}$ & $\mathrm{C}$ & & & $\mathrm{F}$ & $F$ & C & $\mathrm{R}$ & & & & & & C & $\mathbf{R}$ & C & & & & & & $\mathrm{C}$ & $\mathrm{C}$ & $\mathbf{R}$ & C & $\mathrm{C}$ & $\mathbf{R}$ & $\mathrm{C}$ & $\mathrm{F}$ & C \\
\hline 17 & $12 \mathrm{c}$ & $2 \mathrm{H}-3,130$ & 12.80 & A & G & EO & Oo & & C & & & $\mathrm{R}$ & $F$ & $\mathrm{C}$ & $\mathbf{R}$ & C & $\mathrm{R}$ & & & & C & $\mathrm{R}$ & C & $\mathbf{R}$ & & & $\mathrm{R}$ & & C & C & & C & C & & C & $\mathrm{R}$ & C \\
\hline & $12 \mathrm{~b}$ & $2 \mathrm{H}-4,130$ & 14.30 & A & G & E1 & $\mathrm{OO}$ & & $\mathrm{C}$ & & & $F$ & C & $\mathrm{C}$ & $\mathbf{R}$ & $\mathrm{C}$ & $\mathrm{C}$ & & & & $\mathrm{C}$ & & $\mathrm{C}$ & $\mathrm{C}$ & & & $\mathrm{R}$ & & $\mathrm{C}$ & $\mathrm{C}$ & & C & $\mathrm{F}$ & & $\mathrm{C}$ & $\mathrm{R}$ & $\mathrm{F}$ \\
\hline 16 & $12 \mathrm{a}$ & $\begin{array}{l}2 \mathrm{H}-5,130 \\
2 \mathrm{H}-6,40 \\
2 \mathrm{H}-\mathrm{CC} \\
3 \mathrm{H}-1,130 \\
3 \mathrm{H}-2,70 \\
3 \mathrm{H}-\mathrm{CC}\end{array}$ & $\begin{array}{l}15.80 \\
16.40 \\
18.00 \\
\\
\\
27.50\end{array}$ & $\begin{array}{l}\mathrm{A} \\
\mathrm{A} \\
\mathrm{A} \\
\mathrm{A} \\
\mathrm{A} \\
\mathrm{A}\end{array}$ & \begin{tabular}{l|}
$\mathrm{G}$ \\
$\mathrm{M}$ \\
$\mathrm{G}$ \\
$\mathrm{M}$ \\
$\mathrm{M}$ \\
$\mathrm{M}$
\end{tabular} & $\begin{array}{l}\text { E1 } \\
\text { E1 } \\
\text { E1 } \\
\text { E1 } \\
\text { E1 } \\
\text { E1 }\end{array}$ & $\begin{array}{l}\mathrm{O} 1 \\
\mathrm{O} 2 \\
\mathrm{O} 1 \\
\mathrm{O} 2 \\
\mathrm{O} 2 \\
\mathrm{O} 2\end{array}$ & & $\begin{array}{l}\mathrm{C} \\
\mathrm{C} \\
\mathrm{C} \\
\mathrm{C} \\
\mathrm{C} \\
\mathrm{C}\end{array}$ & & & $\begin{array}{l}\mathbf{R} \\
\mathbf{R}\end{array}$ & $\begin{array}{l}F \\
F \\
R \\
F \\
R \\
F\end{array}$ & $\begin{array}{l}C \\
C \\
C \\
C \\
C \\
C\end{array}$ & $\begin{array}{l}\mathrm{R} \\
\mathrm{R} \\
\mathrm{R}\end{array}$ & $\begin{array}{l}\mathrm{C} \\
\mathrm{C} \\
\mathrm{C} \\
\mathrm{C} \\
\mathrm{C} \\
\mathrm{C}\end{array}$ & \begin{tabular}{l|} 
C \\
$C$ \\
$C$ \\
$C$ \\
$C$ \\
$C$
\end{tabular} & $\begin{array}{l}\mathrm{R} \\
\mathrm{C} \\
\mathrm{F} \\
\mathrm{F} \\
\mathrm{R}\end{array}$ & $\begin{array}{l}\mathrm{F} \\
\mathrm{F} \\
\mathrm{F} \\
\mathrm{R} \\
\mathrm{R} \\
\mathrm{C}\end{array}$ & $\mathrm{R}$ & $\begin{array}{l}\text { C } \\
\text { C } \\
\text { C } \\
\text { C } \\
\text { C } \\
\text { C }\end{array}$ & $\mathbf{R}$ & $\begin{array}{l}\text { C } \\
C \\
C \\
F \\
F \\
F\end{array}$ & $\begin{array}{l}R \\
R\end{array}$ & $\begin{array}{l}\mathrm{R} \\
\mathrm{R} \\
\mathrm{R} \\
\mathrm{F} \\
\mathrm{F} \\
\mathrm{F}\end{array}$ & $\begin{array}{l}\mathrm{F} \\
\mathrm{R}\end{array}$ & $\begin{array}{l}\mathrm{R} \\
\mathrm{R}\end{array}$ & $\begin{array}{l}F \\
F\end{array}$ & $\begin{array}{l}\mathrm{C} \\
\mathrm{C} \\
\mathrm{C} \\
\mathrm{C} \\
\mathrm{C} \\
\mathrm{C}\end{array}$ & $\begin{array}{l}\mathrm{C} \\
\mathrm{C} \\
\mathrm{C} \\
\mathrm{C} \\
\mathrm{C} \\
\mathrm{C}\end{array}$ & $\mathbf{R}$ & $\begin{array}{l}\text { C } \\
C \\
C \\
C \\
C \\
C\end{array}$ & $\begin{array}{l}\mathrm{F} \\
\mathrm{R} \\
\mathrm{R} \\
\mathrm{F} \\
\mathrm{R} \\
\mathrm{R}\end{array}$ & $\mathbf{R}$ & $\begin{array}{l}\text { C } \\
C \\
C \\
C \\
C \\
F\end{array}$ & \begin{tabular}{l|}
$\mathrm{R}$ \\
$\mathrm{R}$ \\
$\mathrm{R}$ \\
$\mathrm{R}$ \\
$\mathrm{R}$ \\
$\mathrm{R}$
\end{tabular} & $\begin{array}{l}\mathrm{R} \\
\mathrm{F} \\
\mathrm{F} \\
\mathrm{C} \\
\mathrm{F} \\
\mathrm{F}\end{array}$ \\
\hline
\end{tabular}

Note: For an explanation of the abundance and preservation codes, see the text. For genus names, see Appendix. 
Table 4. Summary of the position of calcareous nannofossil events at Hole 705A.

\begin{tabular}{|c|c|c|}
\hline Event & $\begin{array}{l}\text { Core, section } \\
\text { interval }(\mathrm{cm})\end{array}$ & $\begin{array}{l}\text { Depth } \\
\text { (mbsf) }\end{array}$ \\
\hline Gephyrocapsa spp. $>5.5 \mu \mathrm{m}$ FO & $115-705 \mathrm{~A}-2 \mathrm{H}-1,130 / 115-705 \mathrm{~A}-1 \mathrm{H}-\mathrm{CC}$ & $9.8-8.5$ \\
\hline C. macintyrei LO & $115-705 \mathrm{~A}-2 \mathrm{H}-1,130 / 115-705 \mathrm{~A}-1 \mathrm{H}-\mathrm{CC}$ & $9.8-8.5$ \\
\hline G. oceanica FO & $115-705 \mathrm{~A}-2 \mathrm{H}-2,130 / 115-705 \mathrm{~A}-2 \mathrm{H}-1,130$ & $11.3-9.8$ \\
\hline D. brouweri LO & $115-705 \mathrm{~A}-2 \mathrm{H}-2,130 / 115-705 \mathrm{~A}-2 \mathrm{H}-1,130$ & $11.3-9.8$ \\
\hline D. triradiatus LO & $115-705 \mathrm{~A}-2 \mathrm{H}-2,130 / 115-705 \mathrm{~A}-2 \mathrm{H}-1,130$ & $11.3-9.8$ \\
\hline D. pentaradiatus 10 & $115-705 \mathrm{~A}-2 \mathrm{H}-3,130 / 115-705 \mathrm{~A}-2 \mathrm{H}-2,130$ & $12.8-11.3$ \\
\hline D. surculus LO & $115-705 \mathrm{~A}-2 \mathrm{H}-4,130 / 115-705 \mathrm{~A}-2 \mathrm{H}-3,130$ & $14.3-12.8$ \\
\hline D. tamalis LO & $115-705 \mathrm{~A}-2 \mathrm{H}-5,130 / 115-705 \mathrm{~A}-2 \mathrm{H}-4,130$ & $15.8-14.3$ \\
\hline
\end{tabular}

Note: $L O=$ last occurrence and FO $=$ first occurrence.

Table 6. Summary of the position of calcareous nannofossil events at Hole 707A.

\begin{tabular}{|c|c|c|}
\hline Event & $\begin{array}{l}\text { Core, section, } \\
\text { interval }(\mathrm{cm})\end{array}$ & $\begin{array}{l}\text { Depth } \\
\text { (mbsf) }\end{array}$ \\
\hline C. macintyrei LO & $115-707 \mathrm{~A}-2 \mathrm{H}-3,30 / 115-707 \mathrm{~A}-2 \mathrm{H}-2,130$ & $9.9-9.4$ \\
\hline G. oceanica FO & $115-707 \mathrm{~A}-2 \mathrm{H}-3,130 / 115-707 \mathrm{~A}-2 \mathrm{H}-3,90$ & $10.9-10.5$ \\
\hline D. brouweri LO & $115-707 \mathrm{~A}-2 \mathrm{H}-4,130 / 115-707 \mathrm{~A}-2 \mathrm{H}-3,130$ & $12.4-10.9$ \\
\hline D. triradiatus LO & $115-707 \mathrm{~A}-2 \mathrm{H}-4,130 / 115-707 \mathrm{~A}-2 \mathrm{H}-3,130$ & $12.4-10.9$ \\
\hline D. pentaradiatus LO & $115-707 \mathrm{~A}-2 \mathrm{H}-\mathrm{CC} / 115-707 \mathrm{~A}-2 \mathrm{H}-6,130$ & $16.2-15.4$ \\
\hline D. surculus LO & $115-707 \mathrm{~A} \cdot 3 \mathrm{H}-1,90 / 115-707 \mathrm{~A} \cdot 2 \mathrm{H}-\mathrm{CC}$ & $17.1-16.2$ \\
\hline D. tamalis LO & $115-707 \mathrm{~A}-3 \mathrm{H}-2,90 / 115-707 \mathrm{~A}-3 \mathrm{H}-1,90$ & $18.6-17.1$ \\
\hline Sphenoliths LO & $115-707 \mathrm{~A}-4 \mathrm{H}-1,30 / 115-707 \mathrm{~A}-3 \mathrm{H}-4,30$ & $26.1-21.0$ \\
\hline R. pseudoumbilicus LO & $115-707 \mathrm{~A}-4 \mathrm{H}-2,30 / 115-707 \mathrm{~A}-4 \mathrm{H}-1,30$ & $27.6-26.1$ \\
\hline Amaurolithus spp. LO & $115-707 \mathrm{~A}-5 \mathrm{H}-4,130 / 115-707 \mathrm{~A}-5 \mathrm{H}-3,130$ & $41.3-39.8$ \\
\hline C. acutus LO & $115-707 \mathrm{~A}-6 \mathrm{H}-1,130 / 115-707 \mathrm{~A}-5 \mathrm{H}-\mathrm{CC}$ & $46.4-45.1$ \\
\hline C. rugosus FO & $115-707 \mathrm{~A}-6 \mathrm{H}-1,130 / 115-707 \mathrm{~A}-5 \mathrm{H}-\mathrm{CC}$ & $46.4-45.1$ \\
\hline C. acutus FO & $115-707 \mathrm{~A}-6 \mathrm{H}-3,130 / 115-707 \mathrm{~A}-6 \mathrm{H}-2,40$ & $49.4-47.0$ \\
\hline T. rugosus LO & $115-707 \mathrm{~A}-6 \mathrm{H}-3,130 / 115-707 \mathrm{~A}-6 \mathrm{H}-2,40$ & $49.4-47.0$ \\
\hline D. quinqueramus Lo & $115-707 \mathrm{~A}-6 \mathrm{H}-7,40 / 115-707 \mathrm{~A}-6 \mathrm{H}-6,40$ & $54.5-53.0$ \\
\hline A. amplificus LO & $115-707 \mathrm{~A}-7 \mathrm{H}-5,130 / 115-707 \mathrm{~A}-7 \mathrm{H}-4,130$ & $62.0-60.5$ \\
\hline A. amplificus FO & $115-707 \mathrm{~A}-8 \mathrm{H}-6,130 / 115-707 \mathrm{~A}-8 \mathrm{H}-5,130$ & $73.1-71.6$ \\
\hline Amaurolithus spp. FO & $115-707 \mathrm{~A}-9 \mathrm{H}-5,130 / 115-707 \mathrm{~A}-9 \mathrm{H}-4,130$ & $81.3-79.8$ \\
\hline D. berggrenii $\mathrm{FO}$ & $115-707 \mathrm{~A}-10 \mathrm{H}-4,130 / 115-707 \mathrm{~A}-10 \mathrm{H}-3,130$ & $89.4-87.9$ \\
\hline M. convallis FO & $115-707 \mathrm{~A}-12 \mathrm{H}-2,130 / 115-707 \mathrm{~A}-12 \mathrm{H}-1,130$ & $105.6-104.1$ \\
\hline D. hamatus 10 & $115-707 \mathrm{~A}-12 \mathrm{H}-2,130 / 115-707 \mathrm{~A}-12 \mathrm{H}-1,130$ & $105.6-104.1$ \\
\hline D. hamatus FO & $115-707 \mathrm{~A}-13 \mathrm{H}-1,130 / 115-707 \mathrm{~A}-13 \mathrm{H}-1,40$ & $113.7-112.8$ \\
\hline Catinaster spp. FO & $115-707 \mathrm{~A}-13 \mathrm{H}-3,130 / 115-707 \mathrm{~A}-13 \mathrm{H}-3,40$ & $116.7-115.8$ \\
\hline C. nitescens bo & $115-707 \mathrm{~A}-13 \mathrm{H}-5,40 / 115-707 \mathrm{~A}-13 \mathrm{H}-4,130$ & $118.8-118.2$ \\
\hline D. kugleri FO & ND & \\
\hline C. floridanus LO & $115-707 \mathrm{~A}-13 \mathrm{H}-\mathrm{CC} / 115-70$ & $122.0-119.7$ \\
\hline S. heteromorphus LO & $115-707 \mathrm{~A}-14 \mathrm{H}-1,130 / 115$ & $123.3-122.0$ \\
\hline R. pseudoumbilicus FO & $115-707 \mathrm{~A}-14 \mathrm{H}-2,130 / 115-707 \mathrm{~A}-14 \mathrm{H}-1,130$ & $124.8-123.3$ \\
\hline H. ampliaperta & Present in Sample 115-707A-15H-1, 130 (132.9 mbsf) & \\
\hline D. signus & Present in Sample 115-707 A-14H-3, 130 (126.3 mbsf) & \\
\hline $\begin{array}{l}\text { Reversal massive/free- } \\
\text { ray discoasters }\end{array}$ & $115-707 \mathrm{~A}-14 \mathrm{H}-4,130 / 115-707 \mathrm{~A}-14 \mathrm{H}-3,130$ & 127 \\
\hline S. heteromorphus FO & . 130 & $134.4-132.9$ \\
\hline S. belemnas LO & $115-707 \mathrm{~A}-15 \mathrm{H}-2,130 / 115-707 \mathrm{~A}-15 \mathrm{H}-1,130$ & $134.4-132.9$ \\
\hline S. belemnos FO & $115-707 \mathrm{~A}-15 \mathrm{H}-4,90 / 115-707 \mathrm{~A}-15 \mathrm{H}-4,30$ & $137.0-136.4$ \\
\hline T. carinatus LO & $115-707 \mathrm{~A}-15 \mathrm{H}-4,90 / 115-707 \mathrm{~A}-15 \mathrm{H}-4,30$ & $137.0-136.4$ \\
\hline D. druggii $\mathrm{FO}$ & $115-707 \mathrm{~A}-15 \mathrm{H}-4,90 / 115-707 \mathrm{~A}-15 \mathrm{H}-4,30$ & $137.0-136.4$ \\
\hline Z. bijugatus LO & $115-707$ A- $15 \mathrm{H}-5,90 / 115-707 \mathrm{~A}-15 \mathrm{H}-4,130$ & $138.5-137.4$ \\
\hline C. abisectus LO & $115-707 \mathrm{~A}-16 \mathrm{H}-1,40 / 115-707 \mathrm{~A}-15 \mathrm{H}-\mathrm{CC}$ & $141.7-141.3$ \\
\hline S. ciperoensis LO & $115-707 \mathrm{~A}-16 \mathrm{H}-1,40 / 115-707 \mathrm{~A}-15 \mathrm{H}-\mathrm{CC}$ & $141.7-141.3$ \\
\hline D. bisectus LO & $115-707 \mathrm{~A}-16 \mathrm{H}-1,40 / 115-707 \mathrm{~A}-15 \mathrm{H}-\mathrm{CC}$ & $141.7-141.3$ \\
\hline H. recta LO & Missing & \\
\hline
\end{tabular}

Note: $L O=$ last occurrence, FO $=$ first occurrence, and ND $=$ not detectable.

Mascarene Plateau (Fig. 1 and Table 1). Three holes were continuously cored at Site 709. Hole 709A ended in nannofossil ooze of late Oligocene age at $203.1 \mathrm{~m}$ below seafloor (mbsf). Hole 709B ended at 254.8 mbsf in Oligocene oozes, and Hole $709 \mathrm{C}$ in nannofossil oozes of middle Eocene age at $353.7 \mathrm{mbsf}$.

Shipboard analysis has indicated that the three sequences retrieved are virtually identical. Events determined in the three holes are reported in Tables 7, 8, and 9. Interhole correlations based on selected calcareous nannofossil events are shown in Figure 4. For our shore-based study, we have concentrated on the late Pliocene record of Hole 709B (Table 10) and on the latest Oligocene through early Pliocene record of Hole 709C (Table 11, microfiche, back pocket). The Miocene-Pliocene sediments at Site 709 are fairly homogeneous, comprising a single lithologic unit that consists of alternate clay-bearing nannofossil ooze and nannofossil ooze. This unit was divided into subunits on the basis of distinctive changes in color (Fig. 5).

The calcareous nannofossil biostratigraphy in a composite Hole 709A and Hole 709B section is presented in Figure 5. Calcareous nannofossils are abundant throughout the sequence, with varying preservational states. Overgrowth in discoasters is present, but it does not represent a major problem for the recognition of marker species. Etching is never very strong, but the assemblage is enriched in dissolution-resistant species, as indicated by the discoasterid abundances (Fig. 5), intermediate between those observed at Site 707 (Fig. 3) and those detected at deeper water CBT sites.

As shown in Tables 10 and 11, the biostratigraphic interpretation is not immediately apparent because of the generally strong reworking that characterizes most of the sequence and because of anomalous stratigraphic inversions particularly evident in the early and middle Miocene (Fig. 6), where assemblages indicative of Zone $\mathrm{CN} 2$ are intercalated within sediments belonging to Zone $\mathrm{CN} 3$ and Zone CN4. Often there is little or no detectable mixing in these assemblages. These stratigraphic inversions are not associated with sedimentologic evidence of turbidites or of slumping (Backman, Duncan, et al., 1988), and they may represent cryptoturbidites triggered by intensified bottom currents or by tectonic instability associated with possible increased seismicity.

Besides intense redeposition, the sequence is also affected by several hiatuses. A first short hiatus occurs at about $20 \mathrm{mbsf}$, where we detected the LO of $D$. brouweri, the FO of $G$. ocean$i c a$ and the LO of $C$. macintyrei within a 1.5 -m-thick interval (Table 8). The three events are spaced apart from each other in the other sites, spanning a time interval of about $0.4 \mathrm{~m} . \mathrm{y}$. (Table 2).

Another interval of compressed sedimentation and hiatuses occurs at the transition from the middle Miocene to the late Miocene in Core 115-709C-13H (Fig. 5), where the LO of D. hamatus (about $8.67 \mathrm{Ma}$ ) is recorded only $3-4 \mathrm{~m}$ above the FO of C. coalitus (about $11.1 \mathrm{Ma}$ ).

As in all the other CBT sites, Zones CN2 and NN3 were not detected, and we interpret this finding as caused by a generalized hiatus in the area.

\section{Site 710}

Site 710 is located in a fairly broad flat northeast-trending terrace, adjacent to elevated grounds. It is located only $30 \mathrm{nmi}$ from Site 709 , which is about $800 \mathrm{~m}$ shallower. The water depth of Site 710 is 3810 mbsf, close to the present day sedimentary lysocline; therefore, it is well suited for studying carbonate flux and dissolution. Two holes were continuously cored at Site 710 . Hole 710A ended in nannofossil ooze-chalk of early Oligocene age at 209.7 mbsf. Hole $710 \mathrm{~B}$ recovered a duplicate of the late Neogene sequence and ended in clay nannofossil ooze and nannofossil clay of late Miocene age, at 83.4 mbsf.

Site 710 contains the first magnetostratigraphic record obtained during Leg 115, the interpretation of which implies major changes in the generally accepted middle Miocene calcareous nannofossil biochronology (Backman et al., this volume) as commented on previously. Calcareous nannofossils are generally abundant and are scarce or absent in only a few samples in the late middle Miocene (Table 12, microfiche, back pocket).

The preservation state is variable, and this variability can be explained as caused by fluctuations of the CCD and by the redeposition of carbonate material from shallower water areas. Strong reworking and stratigraphic inversions are present in the sequence as discussed below and documented in the range charts (Tables 12 and 13, microfiche, back pocket).

The two holes were correlated by means of biostratigraphy and magnetostratigraphy (Fig. 7). A composite section of the 


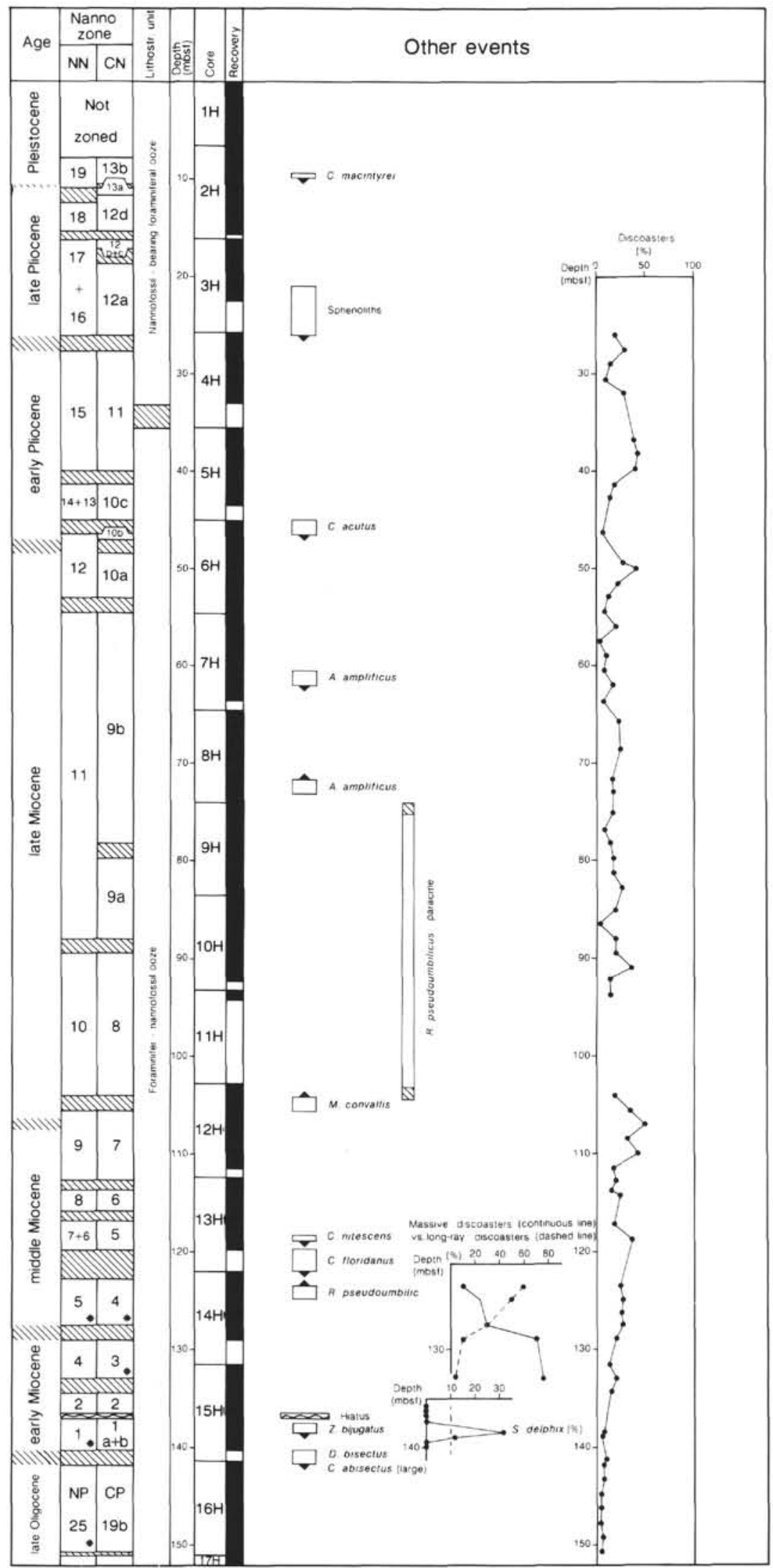

Figure 3. Chronostratigraphy and calcareous nannofossil biostratigraphy at Hole 707A. Striped intervals at boundaries in zonal column represent uncertainties introduced by sample spacing. Black in recovery column represents recovered intervals. ${ }^{*}$ represents redefined boundary concepts. 
Table 7. Summary of the position of calcareous nannofossil events at Hole 709A.

\begin{tabular}{llc}
\hline \multicolumn{1}{c}{ Event } & \multicolumn{1}{c}{$\begin{array}{c}\text { Core, section, } \\
\text { interval }(\mathrm{cm})\end{array}$} & $\begin{array}{c}\text { Depth } \\
\text { (mbsf) }\end{array}$ \\
\hline & \multicolumn{3}{c}{. } & \\
C. macintyrei LO & $115-709 \mathrm{~A}-3 \mathrm{H}-1,35 / 115-709 \mathrm{~A}-2 \mathrm{H}-\mathrm{CC}$ & $20.05-19.7$ \\
D. brouweri LO & $115-709 \mathrm{~A}-3 \mathrm{H}-1,35 / 115-709 \mathrm{~A}-2 \mathrm{H}-\mathrm{CC}$ & $20.05-19.7$ \\
D. triradiatus LO & $115-709 \mathrm{~A}-3 \mathrm{H}-1,35 / 115-709 \mathrm{~A}-2 \mathrm{H}-\mathrm{CC}$ & $20.05-19.7$ \\
D. pentaradiatus LO & $115-709 \mathrm{~A}-3 \mathrm{H}-4,90 / 115-709 \mathrm{~A}-3 \mathrm{H}-4,35$ & $25.1-24.55$ \\
R. pseudoumbilicus LO & $115-709 \mathrm{~A}-4 \mathrm{H}-4,140 / 115-709 \mathrm{~A}-4 \mathrm{H}-4,60$ & $35.30-34.5$ \\
C. rugosus FO & $115-709 \mathrm{~A}-6 \mathrm{H}-6,130 / 115-709 \mathrm{~A}-6 \mathrm{H}-5,30$ & $57.0-55.0$ \\
C. acutus FO & $115-709 \mathrm{~A}-7 \mathrm{H}-1,130 / 115-709 \mathrm{~A}-7 \mathrm{H}-1,45$ & $59.6-58.75$ \\
D. quinqueramus LO & $115-709 \mathrm{~A}-7 \mathrm{H}-5,42 / 115-709 \mathrm{~A}-7 \mathrm{H}-3,42$ & $64.72-61.72$ \\
Amaurolithus spp. FO & $115-709 \mathrm{~A}-10 \mathrm{H}-\mathrm{CC} / 115-709 \mathrm{~A}-10 \mathrm{H}-3,130$ & $96.9-91.6$ \\
M. convallis FO & $115-709 \mathrm{~A}-12 \mathrm{H}-3,130 / 115-709 \mathrm{~A}-12 \mathrm{H}-3,45$ & $110.9-110.05$ \\
D. hamatus LO & $115-709 \mathrm{~A}-12 \mathrm{H}-3,130 / 115-709 \mathrm{~A}-12 \mathrm{H}-3,45$ & $110.9-110.05$ \\
D. hamatus FO & $115-709 \mathrm{~A}-12 \mathrm{H}-6,130 / 115-709 \mathrm{~A}-12 \mathrm{H}-6,45$ & $115.4-114.55$ \\
C. coalitus FO & $115-709 \mathrm{~A}-13 \mathrm{H}-1,45 / 115-709 \mathrm{~A}-12 \mathrm{H}-\mathrm{CC}$ & $116.65-116.2$ \\
S. ciperoensis LO & $115-709 \mathrm{~A}-21 \mathrm{H}-4,45 / 115-709 \mathrm{~A}-21 \mathrm{H}-3,130$ & $198.45-197.8$ \\
\hline
\end{tabular}

Note: $L O=$ last occurrence and FO = first occurrence.

Table 8. Summary of the position of calcareous nannofossil events at Hole 709B.

\begin{tabular}{|c|c|c|}
\hline Event & $\begin{array}{l}\text { Core, section, } \\
\text { interval }(\mathrm{cm})\end{array}$ & $\begin{array}{l}\text { Depth } \\
\text { (mbsf) }\end{array}$ \\
\hline C. macintyrei LO & $115-709 \mathrm{~B}-3 \mathrm{H}-5,0 / 115-709 \mathrm{~B}-2 \mathrm{H}-7,52$ & $19.4-10.32$ \\
\hline G. oceanica FO & $115-709 \mathrm{~B}-3 \mathrm{H}-5,0 / 115-709 \mathrm{~B}-2 \mathrm{H}-7,52$ & $19.4-10.32$ \\
\hline D. brouweri LO & $115-709 \mathrm{~B}-3 \mathrm{H}-6,2 / 115-709 \mathrm{~B}-3 \mathrm{H}-5,0$ & $20.92-19.4$ \\
\hline D. triradiatus LO & $115-709 \mathrm{~B}-3 \mathrm{H}-6-2 / 115-709 \mathrm{~B}-3 \mathrm{H}-5,0$ & $20.92-19.4$ \\
\hline D. pentaradiatus LO & $115-709 \mathrm{~B}-3 \mathrm{H}-7,60 / 115-709 \mathrm{~B}-3 \mathrm{H}-6,100$ & $23.0-21.90$ \\
\hline D. surculus LO & $115-709 \mathrm{~B}-4 \mathrm{H}-1,90 / 115-709 \mathrm{~B}-4 \mathrm{H}-1,60$ & $24.0-23.70$ \\
\hline D. tamalis LO & II5-709B-4H-4, 90/115-709B-4H-4, 30 & $28.50-27.30$ \\
\hline Sphenoliths LO & $115-709 \mathrm{~B}-5 \mathrm{H}-1-120 / 115-709 \mathrm{~B}-5 \mathrm{H}-1,60$ & $34.0-33.40$ \\
\hline R. pseudoumbilicus LO & $115-709 \mathrm{~B}-5 \mathrm{H}-3-50 / 115-709 \mathrm{~B}-5 \mathrm{H}-2,120$ & $36.30-35.50$ \\
\hline C. rugosus $\mathrm{FO}$ & $115-709 \mathrm{~B}-7 \mathrm{H}-2,130 / 115-709 \mathrm{~B}-7 \mathrm{H}-1,130$ & $54.80-53.30$ \\
\hline C. acutus $\mathrm{FO}$ & $115-709 \mathrm{~B}-7 \mathrm{H}-4,130 / 115-709 \mathrm{~B}-7 \mathrm{H}-3,130$ & $57.80-56.30$ \\
\hline D. quinqueramus LO & 115-709B-8H-1, 130/115-709B-7H-6, 130 & $63.0-60.80$ \\
\hline Amaurolithus spp. FO & $115-709 \mathrm{~B}-11 \mathrm{H}-1,130 / 115-709 \mathrm{~B}-10 \mathrm{H}-7,20$ & $91.9-90.2$ \\
\hline D. berggrenii FO & $115-709 \mathrm{~B}-12 \mathrm{H}-5,130 / 115-709 \mathrm{~B}-12 \mathrm{H}-3,130$ & $107.60-104.60$ \\
\hline D. hamatus LO & $115-709 \mathrm{~B}-13 \mathrm{H}-1,130 / 115-709 \mathrm{~B}-12 \mathrm{H}-6,130$ & $111.20-109.10$ \\
\hline D. hamatus FO & $115-709 \mathrm{~B}-13 \mathrm{H}-3,130 / 115-709 \mathrm{~B}-13 \mathrm{H}-2,130$ & $114.20-112.70$ \\
\hline C. coalitus $\mathrm{FO}$ & $115-709 \mathrm{~B}-13 \mathrm{H}-4,130 / 115-709 \mathrm{~B}-13 \mathrm{H}-3,130$ & $115.70-114.20$ \\
\hline S. ciperoensis LO & $115-709 \mathrm{~B}-22 \mathrm{X}-3,130 / 115-709 \mathrm{~B}-22 \mathrm{X}-1,130$ & $201.1-198.1$ \\
\hline
\end{tabular}

Note: $\mathrm{LO}=$ last occurrence and $\mathrm{FO}=$ first occurrence.

two holes is shown in Figure 8. The two sequences retrieved differ substantially in the upper part (Fig. 7). In Hole 710A biostratigraphy and magnetostratigraphy indicate a hiatus between Core $115-710 \mathrm{~A}-2 \mathrm{H}$ and Core $115-710 \mathrm{~A}-3 \mathrm{H}$, which removed the sediments of the early Matuyama Chron. In Hole 710B the Pleistocene sequence is more expanded (although problems of overcoring are suspected; Backman, Duncan, et al., 1988) and a hiatus is present between Core 115-710B-3H, in which the basal sediments are above the Olduvai Subchron $(G$. oceanica is present and $C$. macintyrei is missing), and Core 115-710B-4H, in which the top sediments belong to the lower-middle Gauss Chron, as indicated by the presence of $D$. tamalis. Most probably the two holes were drilled in an area where very localized scour erosion was active in the late Pliocene and early Pleistocene.

The intervals underlying these hiatuses appear correlatable, even if the correlations shown in Figure 7 indicate the presence of minor offsets and missing sediments across core boundaries (Tables 14 and 15). Note, as an example, the polarity pattern of the Gilbert Chron in Holes 710A and 710B. The positive polarity interval associated with the LO of Amaurolithus spp. (Nunivak Subchron) in Hole $710 \mathrm{~A}$ is not detected in Hole $710 \mathrm{~B}$, where it is expected between Cores 115-710B-4H and 115-710B$5 \mathrm{H}$. Apart from these discrepancies, the magnetostratigraphic
Table 9. Summary of the position of calcareous nannofossil events at Hole 709C.

\begin{tabular}{|c|c|c|}
\hline Event & $\begin{array}{l}\text { Core, section, } \\
\text { interval }(\mathrm{cm})\end{array}$ & $\begin{array}{l}\text { Depth } \\
\text { (mbsf) }\end{array}$ \\
\hline D. tamalis LO & $115-709 \mathrm{C}-4 \mathrm{H}-3,60 / 115-709 \mathrm{C}-4 \mathrm{H}-2,60$ & $28.7-27.2$ \\
\hline Sphenoliths LO & $115-709 \mathrm{C}-5 \mathrm{H}-1,60 / 115-709 \mathrm{C}-4 \mathrm{H}-6,60$ & $35.3-33.2$ \\
\hline R. pseudoumbilicus LO & $115-709 \mathrm{C}-5 \mathrm{H}-2,60 / 115-709 \mathrm{C}-5 \mathrm{H}-1,60$ & $36.8-35.3$ \\
\hline Amaurolithus spp. LO & $115-709 \mathrm{C}-6 \mathrm{H}-2,60 / 115-709 \mathrm{C}-6 \mathrm{H}-1,60$ & $46.4-44.9$ \\
\hline C. acutus LO & $115-709 \mathrm{C}-6 \mathrm{H}-6,60 / 115-709 \mathrm{C}-6 \mathrm{H}-5,60$ & $52.4-50.9$ \\
\hline C. rugosus FO & $115-709 \mathrm{C}-6 \mathrm{H}-7,60 / 115-709 \mathrm{C}-6 \mathrm{H}-6,60$ & $53.9-52.4$ \\
\hline C. acutus FO & $115-709 \mathrm{C}-7 \mathrm{H}-4,60 / 115-709 \mathrm{C}-7 \mathrm{H}-3,60$ & $59.0-57.5$ \\
\hline T. rugosus LO & $115-709 \mathrm{C}-7 \mathrm{H}-4,60 / 115-709 \mathrm{C}-7 \mathrm{H}-3,60$ & $59.0-57.5$ \\
\hline D. quinqueramus $\mathrm{LO}$ & $115-709 \mathrm{C}-7 \mathrm{H}-6,60 / 115-709 \mathrm{C}-7 \mathrm{H}-5,60$ & $62.0-60.5$ \\
\hline A. amplificus LO & $115-709 \mathrm{C}-8 \mathrm{H}-5,60 / 115-709 \mathrm{C}-8 \mathrm{H}-4,60$ & $70.2-68.7$ \\
\hline A. amplificus $\mathrm{FO}$ & $115-709 \mathrm{C}-10 \mathrm{H}-1,60 / 115-709 \mathrm{C}-9 \mathrm{H}-6,60$ & $83.5-81.3$ \\
\hline Amaurolithus spp. FO & $115-709 \mathrm{C}-10 \mathrm{H}-6,60 / 115-709 \mathrm{C}-10 \mathrm{H}-5,60$ & $91.0-89.5$ \\
\hline M. convallis LO & $115-709 \mathrm{C}-10 \mathrm{H}-6,60 / 115-709 \mathrm{C}-10 \mathrm{H}-5,60$ & $91.0-89.5$ \\
\hline D. berggrenii $\mathrm{FO}$ & $115-709 \mathrm{C}-12 \mathrm{H}-4,30 / 115-709 \mathrm{C}-12 \mathrm{H}-3,60$ & $107.0-105.8$ \\
\hline M. convallis FO & $115-709 \mathrm{C}-13 \mathrm{H}-2,60 / 115-709 \mathrm{C}-13 \mathrm{H}-1,90$ & $113.9-112.7$ \\
\hline D. hamatus LO & $115-709 \mathrm{C}-13 \mathrm{H}-2,60 / 115-709 \mathrm{C}-13 \mathrm{H}-1,90$ & $113.9-112.7$ \\
\hline C. calyculus FO & $115-709 \mathrm{C}-13 \mathrm{H}-3,60 / 115-709 \mathrm{C}-13 \mathrm{H}-2,90$ & $115.4-114.2$ \\
\hline D. hamatus FO & $115-709 \mathrm{C}-13 \mathrm{H}-3,60 / 115-709 \mathrm{C}-13 \mathrm{H}-2,90$ & $115.4-114.2$ \\
\hline C. coalitus FO & $115-709 \mathrm{C}-13 \mathrm{H}-3,90 / 115-709 \mathrm{C}-13 \mathrm{H}-3,60$ & $115.7-115.4$ \\
\hline D. kugleri FO & $115-709 \mathrm{C}-14 \mathrm{H}-1,30 / 115-709 \mathrm{C}-13 \mathrm{H}-6,90$ & $121.7-120.2$ \\
\hline C. nitescens 10 & $115-709 \mathrm{C}-14 \mathrm{H}-1,30 / 115-709 \mathrm{C}-13 \mathrm{H}-6,90$ & $121.7-120.2$ \\
\hline C. floridanus LO & $115-709 \mathrm{C}-14 \mathrm{H}-3,30 / 115-709 \mathrm{C}-14 \mathrm{H}-2,60$ & $124.7-123.5$ \\
\hline S. heteromorphus LO & $115-709 \mathrm{C}-14 \mathrm{H}-5,30 / 115-709 \mathrm{C}-14 \mathrm{H}-4,60$ & $127.7-126.5$ \\
\hline R. pseudoumbilicus FO & $115-709 \mathrm{C}-14 \mathrm{H}-5,30 / 115-709 \mathrm{C}-14 \mathrm{H}-4,60$ & $127.7-126.5$ \\
\hline H. ampliaperta LO & Missing & \\
\hline D. signus FO & $115-709 \mathrm{C}-16 \mathrm{H}-1,60 / 115-709 \mathrm{C}-15 \mathrm{H}-7,25$ & -140.4 \\
\hline D. deflandrei acme end & $115-709 \mathrm{C}-16 \mathrm{H}-1,60 / 115-709 \mathrm{C}-15 \mathrm{H}-7,25$ & $141.4-140.4$ \\
\hline D. formosus $\mathrm{FO}$ & $115-709 \mathrm{C}-16 \mathrm{H}-6,60 / 115-709 \mathrm{C}-16 \mathrm{H}-5,60$ & $148.9-147.4$ \\
\hline S. heteromorphus FO & $115-709 \mathrm{C}-18 \mathrm{H}-\mathrm{CC} / 115-709 \mathrm{C}-18 \mathrm{H}-7,60$ & $169.9-169.8$ \\
\hline S. belemnos LO & $115-709 \mathrm{C}-18 \mathrm{H}-\mathrm{CC} / 115-709 \mathrm{C}-18 \mathrm{H}-7,60$ & $169.9-169.8$ \\
\hline T. carinatus LO & $115-709 \mathrm{C}-18 \mathrm{H}-\mathrm{CC} / 115-709 \mathrm{C}-18 \mathrm{H}-7,60$ & $169.9-169.8$ \\
\hline S. belemnos FO & $115-709 \mathrm{C}-19 \mathrm{H}-1,60 / 115-709 \mathrm{C}-19 \mathrm{H}-1,30$ & $170.5-170.2$ \\
\hline D. druggii $\mathrm{FO}$ & $115-709 \mathrm{C}-21 \mathrm{X}-3,60 / 115-709 \mathrm{C}-21 \mathrm{X}-2,60$ & $192.7-191.2$ \\
\hline C. abisectus LO & $115-709 \mathrm{C}-21 \mathrm{X}-6,60 / 115-709 \mathrm{C}-21 \mathrm{X}-5,80$ & $197.2-195.9$ \\
\hline Z. bijugatus LO & $115-709 \mathrm{C}-22 \mathrm{X}-1,60 / 115-709 \mathrm{C}-21 \mathrm{X}-\mathrm{CC}$ & 199.3-198.7 \\
\hline S. ciperoensis $\mathrm{LO}$ & $115-709 C-22 X-2,60 / 115-709 C-22 X-1,60$ & $200.8-199.3$ \\
\hline D. bisectus LO & $115-709 C-22 X-3,60 / 115-709 C-22 X-2,60$ & $202.3-200.8$ \\
\hline H. recta LO & Missing & \\
\hline
\end{tabular}

Note: $L O=$ last occurrence and $\mathrm{FO}=$ first occurrence.

correlations, aided by biostratigraphic data (shown in Fig. 7), suggest a complete late Miocene-early Pliocene magnetostratigraphic record, which compares well with the Geomagnetic Polarity Time Scale (GPTS) of Berggren et al. (1985) reported in Figure 2.

\section{Stratigraphic Remarks}

The composite sequence at Site 710, presented in Figure 8, indicates that sedimentation was continuous from the late Pliocene (late Gauss) to the late middle Miocene (Chron C5, at the base of Core 115-710A-7H) below the hiatus commented above. In this interval the indigenous nannofossil assemblages are dissolved, as indicated by the visual estimate of etching, by the low species diversity (Tables 12 and 13) and the high abundances of discoasterids (Fig. 8). This interval is characterized, however, by reworking and displacement of penecontemporaneous shallower water assemblages, as indicated by the strong contrast in the preservation and abundance of discoasterids.

Cores $115-710 \mathrm{~A}-8 \mathrm{H}$ and $-9 \mathrm{H}$ contain a complicated record in which biostratigraphic inversions are present. Specifically, as indicated by quantitative data reported in Figure 9, sediments belonging to Zones CN7 and CN8 are intercalated within Subzone CN9a. These intervals are interpreted as displaced by slumping or turbidites. Core 115-710A-9H has an apparently consistent biomagnetostratigraphic record (Fig. 8), but basal samples from Section 115-710A-9H-7 yield $M$. convallis, $D$. pentaradiatus, and $D$. berggrenii and do not contain $D$. hamatus, thus indicating Zones CN8 and CN9a. It is difficult to offer a definitive in- 
Hole 709A Hole 709B Hole 709C

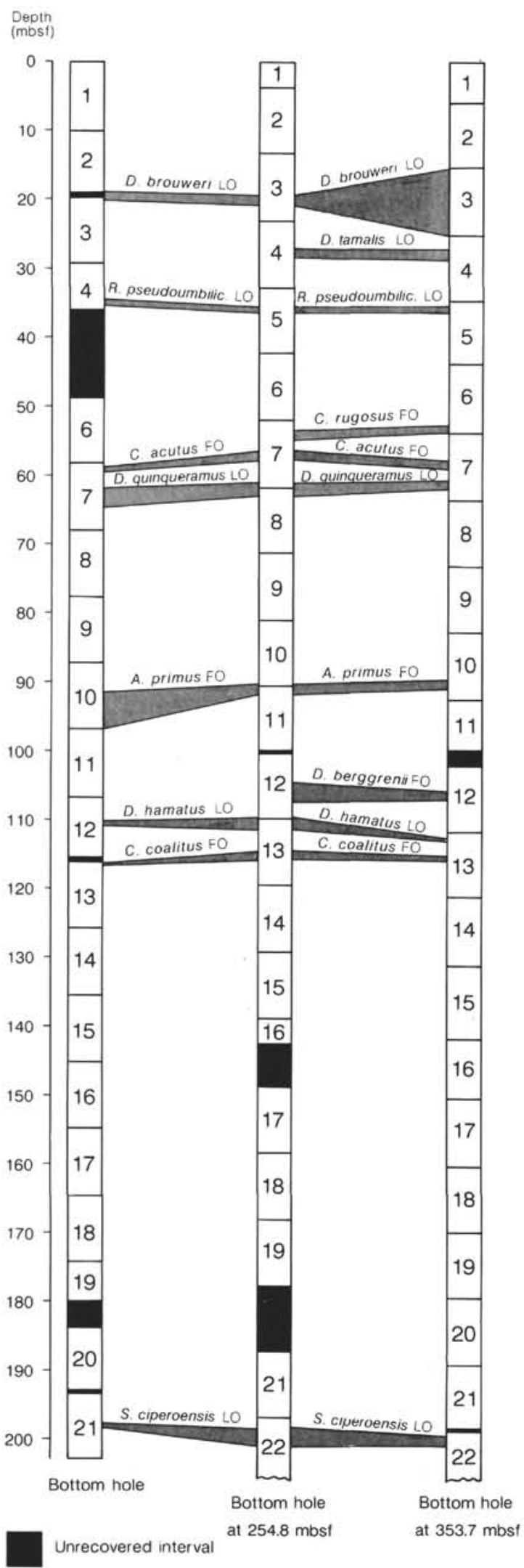

Figure 4. Interhole correlations at Site 709. Black in core columns represents no recovery. terpretation of this finding, which could be caused by downhole displacement.

As in all the other CBT sites, the middle Miocene Zones NN6, NN7, and NN8 are compressed and characterized by strongly dissolved assemblages. The sediment input in this interval is mainly represented by turbidites and displaced material. The top part of Core 115-710A-10H, within Zone NN8, does not contain C. coalitus (Table 12) and is better interpreted as displaced. We recognized all the adopted zones of the early and early middle Miocene (Fig. 2), albeit very compressed, except for Zone NN3 which is missing. In this interval the reworking remains strong, making the recognition of extinction events difficult (Tables 12 and 13).

\section{Magnetobiochronology}

The magnetobiochronology of the Site 710 sequence has been discussed in large part by Backman et al. (this volume). Additional data on late Miocene and Pliocene is shown in Figures 10 and 11 and summarized in Table 2 .

\section{Site 708}

Site 708 is located in the abyssal plain between the Northern Mascarene Plateau and the Carlsberg Ridge, an area of virtually featureless topography (Fig. 1). It is one of the deeper CBT sites (4109 mbsf). A single hole was drilled because of the presence of numerous turbidites. The range chart of calcareous nannofossils at this site is reported in Table 16 (microfiche, back pocket) and the resulting Miocene-Pliocene biostratigraphy is synthesized in Figure 12.

Calcareous nannofossils are present throughout the sequence except in a short interval at about 130 mbsf in the middle Miocene (Table 16 and Fig. 12). However, their abundance and preservation state is strongly variable, as reported in the range chart. Samples that yield assemblages with overgrowth and little etching alternate with others that yield strongly dissolved assemblages. The former samples are interpreted here as representing carbonate material redeposited from shallower water depth areas.

Reworking is very strong throughout the section, and mixed assemblages are common since they are also present in lithologies that were not described as turbidites. Last occurrence datums are obscured by reworking and were picked at the end of the continuous and abundant presence of the marker forms.

The biostratigraphic interpretation (Fig. 12) indicates that all the late middle Miocene to Pleistocene adopted zonal intervals (Fig. 2) are recognizable. Zones NN8, NN9, and NN10 are compressed, in spite of the presence of turbidites. A change in sedimentation rate occurs at the NN10/NN11 boundary. This is a feature present in all the CBT sites.

A megahiatus is present in the late middle Miocene, where Zones NN6 and NN7 are missing. At the top of Core 115-70815X (late middle Miocene) a lithologic slump, with an apparently consistent late Paleocene assemblage, occurs. The slump could possibly signal the initiation of canyon cutting or tectonic instability along the Northern Mascarene Plateau during Zone NN6-NN7 times. All of the underlying adopted Miocene zonal intervals were recognized except for Zones NN3 and CN2.

The abundance of discoasterids is high in the pelagic sediments of the Site 708 sequence starting from the upper part of Zone NN2 (Fig. 12), indicating that strong dissolution was active during most of the Miocene at this site (Table 17). This abundance change occurs slightly above a sharp decrease in carbonate content (Backman, Duncan, et al., 1988).

\section{Site 711}

Site 711 is located on the northern edge of the Madingley Rise, just a few hundred meters above the abyssal plain that separates the Madingley Rise from the Carlsberg Ridge (Fig. 1). 
Table 10. Distribution of calcareous nannofossil taxa at Hole 709B.

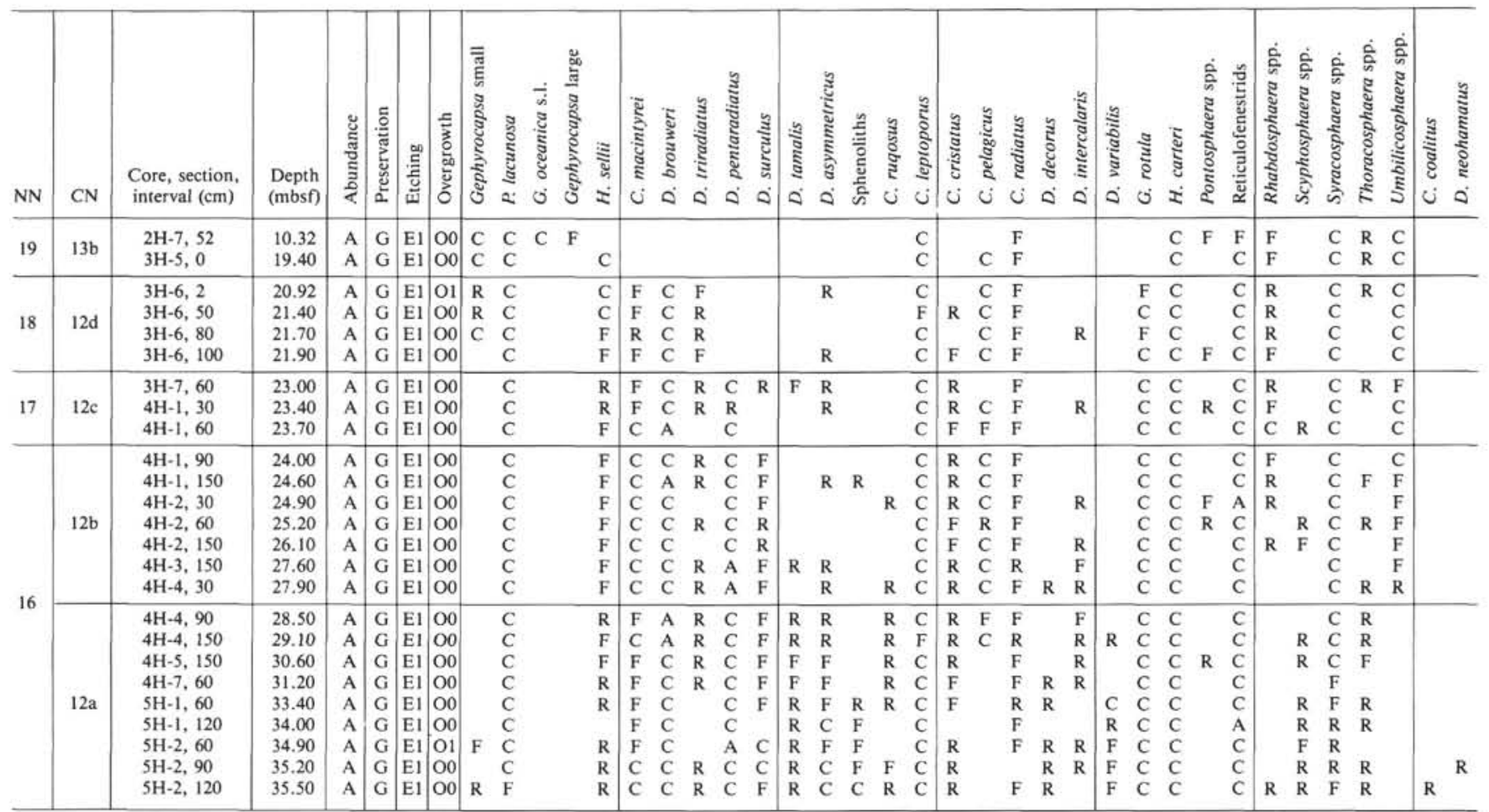

Note: For an explanation of the abundance and preservation codes, see the text. For genus names, see Appendix.

Site 711 forms the deep end-member of the CBT and is located in the present-day sublysocline transition zone. Thus, the sediments at Site 711 are critical in monitoring time-dependent vertical changes in the CCD position.

Two holes were continuously cored at Site 711. Hole 711A ended in radiolarian-bearing nannofossil chalk and banded cherts, middle Eocene in age, at 249.6 mbsf. Hole 711B was cored from the mud line to $98.3 \mathrm{mbsf}$ and ended in clay-bearing nannofossil ooze, late Oligocene in age.

Site 711 was below the CCD through most of the Neogene. The sequence is virtually devoid of foraminifers. Calcareous nannofossils are missing for long intervals and are mainly represented by solution resistant species. A good magnetostratigraphy was obtained in the late Neogene and late Oligocene-early Miocene intervals (Schneider and Kent, this volume).

Both holes were sampled at closely spaced intervals. The positions of biostratigraphic events in the two holes are summarized in Tables 18 and 19. The two sequences retrieved are virtually identical as indicated by the correlations reported in Figure 13. We present here the range chart (Table 20, microfiche, back pocket) of calcareous nannofossils at Hole 711B, which is the best recovered. The only unrecovered interval (Core 115-711B-7H) at this hole should be barren on the base of correlation shown in Figure 13. The numerous barren samples investigated are not shown in the range chart.

A synthesis of the biostratigraphy at this site is presented in Figure 14. Not surprisingly, this deep site is characterized, in spite of the presence of distal turbidites and nannofossil reworking, by distinctly low sedimentation rates. However, despite the strongly compressed sedimentation, this sequence appears to represent continuous sedimentation of residual facies.

Three barren intervals are recognized in the sequence (Figs. 13 and 14). The first barren interval can be precisely dated by means of magnetostratigraphy. The top is very close to a nega- tive polarity interval (Kaena or Mammoth Subchron) of the Gauss Chron at an age of about $3.0 \mathrm{Ma}$; the base of the interval is in the basal Gilbert, just above the LO of $A$. amplificus (about $5.33 \mathrm{Ma}$ ) and has an age of about 5.0 Ma. Within this period of high CCD levels a slight deepening of the CCD is observed for a short interval around the top of the Thvera Subchron (Figs. 13 and 14), where very dissolved nannofossil assemblages, represented only by discoasterids and smoothed ceratoliths, are present.

The second barren interval is comprehended between the very top of Chron C4A, at about $8 \mathrm{Ma}$, and the top of Zone NN5, at about $13 \mathrm{Ma}$ (Fig. 14).

The third barren interval is more difficult to date precisely because it occurs in an interval lacking magnetostratigraphy (Fig. 13). However, it encompasses part of Zone NN4 and the entire Zones $\mathrm{CN} 2$ and NN3 (Fig. 14). It is interesting to note that all three intervals correlate, as discussed below, with hiatuses or strong dissolution at the shallower water CBT Sites 708, 709 , and 710 . These barren intervals indicate true dissolution pulses, caused by shallowing of the CCD.

\section{Preservation Stratigraphy at CBT Sites}

The previous discussion indicates that the stratigraphic records at the CBT sites are not uncomplicated. It seems necessary to summarize the main results obtained about the spatial and temporal distribution of hiatuses and redeposited material and about the variation through time of carbonate dissolution as recorded by the preservation state of calcareous nannofossils. In the search for connections and possible causal mechanisms, we will compare the timing of dissolution events and hiatuses at the CBT sites vs. the major steps of the Neogene climatic evolution.

We are interested here in trends and first-order changes. Higher variability is present in our records, but available data are insufficient for detailed reconstructions. To help the follow- 


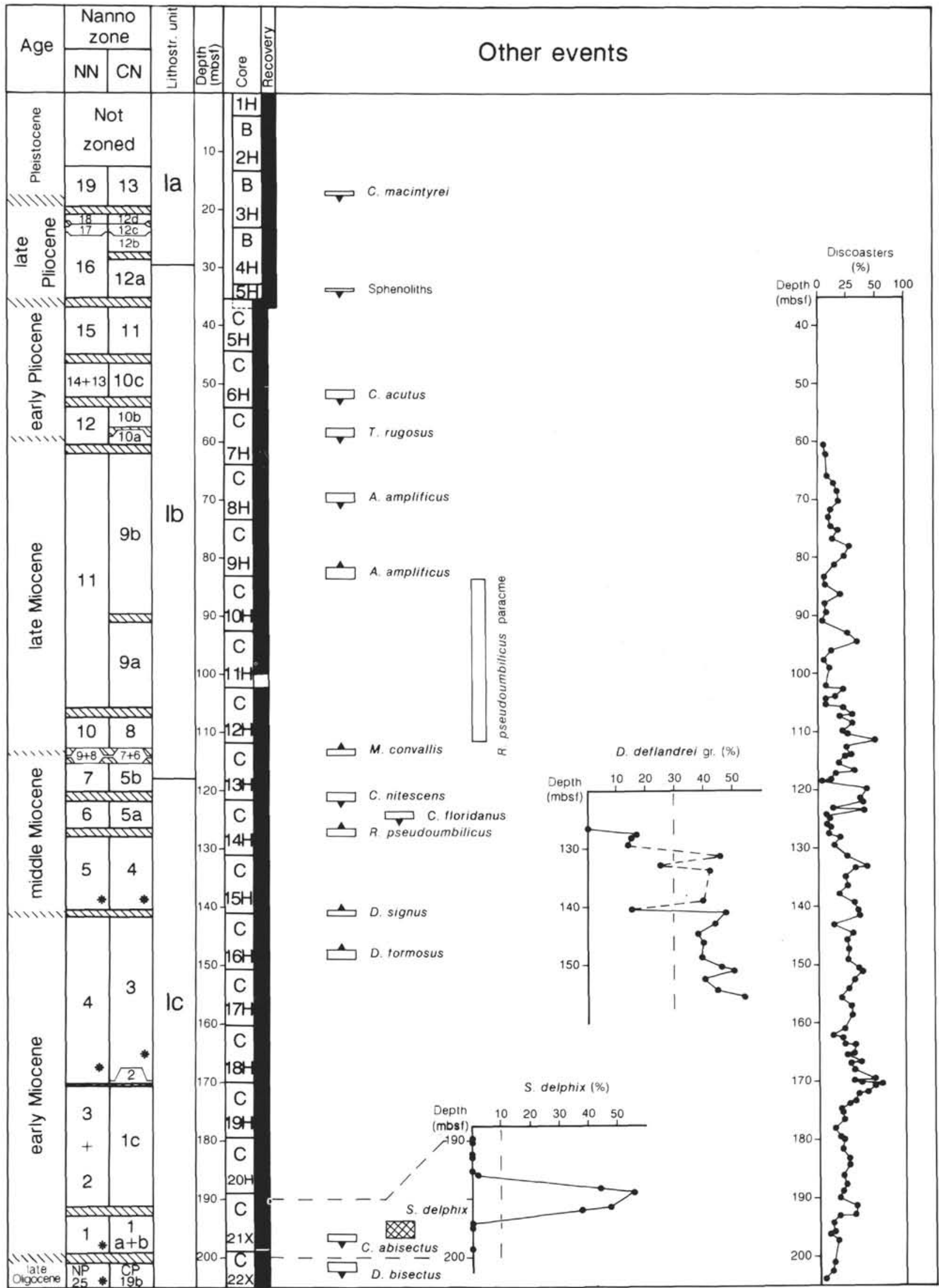

Figure 5. Chronostratigraphy and calcareous nannofossil biostratigraphy at Site 709. For legend, see Figure 3. 


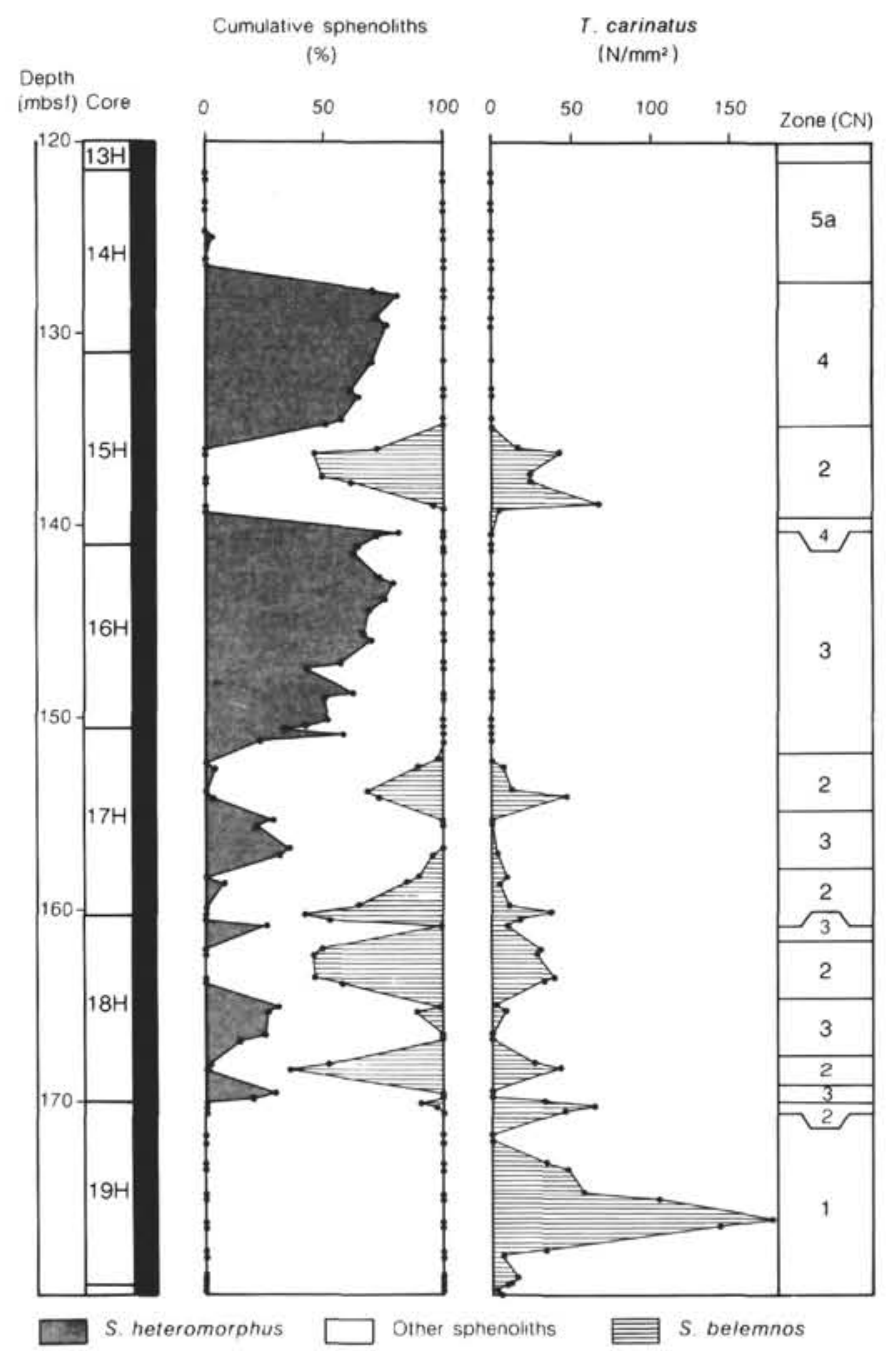

Figure 6. Plots of abundance distributions of $S$. belemnos, S. heteromorphus, and T. carinatus at Hole $709 \mathrm{C}$ showing stratigraphic inversions. Sphenolithid cumulative percentages are based on a count of 100 specimens. The abundance of $T$. carinatus is expressed in the number of specimens found per unit area in the slide.

ing discussion, we have prepared the correlations presented in Figures 15-17 and a summary of the Neogene environmental evolution in Figure 18. In the latter figure, to avoid errors in fitting data based on different biochronologies, we have used the time scale of Keller and Barron (1987), from whom most of the environmental data are deduced. We present our chronicle starting from the early Miocene.

\section{Early Miocene Change in Coccolith Preservation}

Sediments belonging to the late Oligocene and to most of the early Miocene Zone CN1 have a high carbonate content (Backman, Duncan, et al., 1988). Calcareous nannofossils show little etching, and discoasterid abundance is generally low, even in the deep-water sites (Figs. 8 and 14). Sedimentation rates were low at all sites (Fig. 15). The highest sedimentation rate was found at Site 709 (about $30 \mathrm{~m} / 5 \mathrm{~m}$.y.). At shallow-water Site 707, the sediment thickness is reduced $(<10 \mathrm{~m})$ and hiatuses are present. These hiatuses, considering the topographic position of the site, could have been related to current erosion and/or mass wasting. At all other sites, where the carbonate input is represented only by calcareous nannofossils, the low sedimentation rates are con- sidered to be related to dissolution of foraminifers and to low productivity of biogenic carbonate in this time interval.

In the upper part of Subzone $\mathrm{CN} 1 \mathrm{c}$, a change in preservation of calcareous nannofossils occurs in all deep-water sites: etching and discoaster abundance increase at Site 708 (Fig. 12) and 710 (Fig. 8). Zone CN2 is severely compressed or missing. At Site 711 sediments become barren of calcareous nannofossils in the upper part of Subzone CN1c (Fig. 14). All of these findings point to a severe shallowing of the CCD. If we assume that the sharp increase in discoasterid abundance at Site 710 is indicative of this change, we can infer age of about $20 \mathrm{Ma}$ (middle of Chron C6) for this event (Fig. 8).

Looking at the environmental evolution summarized in Figure 18 , the 20 -m.y. change in carbonate sedimentation pattern in the western equatorial Indian Ocean seems to correlate with early Miocene major plate reorganizations. Specifically, the closure of the Tethys and the opening of the Drake passage seemed to have occurred approximately at these times (Fig. 18). These tectonic events could have affected surface and bottom circulation and, hence, fertility and dissolution patterns.

\section{Early-Middle Miocene Amelioration}

Shortly below the acme end of $D$. deflandrei (close to the early/middle Miocene boundary) and up to the LO of S. heteromorphus, dissolved nannofossil assemblages reappear in Site 711 sequence (Fig. 14) and the discoasterid percentage decreases at Site 710 (Fig. 8). These findings are interpreted as indicative of a temporary deepening of the CCD. This temporary amelioration in carbonate preservation seems to occur during times (late $\mathrm{CN} 3$ and $\mathrm{CN} 4$ ) of high sea-level stand and of reduced $\delta^{18} \mathrm{O}$ content in benthic foraminifers (Fig. 18). The light $\delta^{18} \mathrm{O}$ in benthic foraminifers could indicate little ice-cap effect and hence sluggish circulation of less corrosive waters. The high sea-level stand could have caused trapped organic carbon on the shelves, with reduced dissolution in deep-sea sediments (Broecker, 1981, 1982).

At shallow-water Site 707, Zones CN3 and CN4 are compressed (Fig. 15) and probably mass wasting and/or current erosion were still active along the slope, as during the underlying intervals. Instability in the slope during these times is also suggested by the abundance of redeposited material, primarily at Site 709 (Fig. 15).

\section{Middle Miocene Carbonate Crisis}

A major change in carbonate preservation occurs at the CN4/ CN5 boundary (Fig. 16). Nannofossils disappear at Site 711, although a significant increase in discoaster abundance is observed at Site 710 (Fig. 8). These findings point to a severe shallowing of the CCD above the paleodepth of Site 710. This shoaling of the CCD lasted, with minor oscillations, up to the top part of Zone CN8 (just below the FO of $D$. berggrenii), when dissolved assemblages of nannofossils reenter the Site 711 sequence (Fig. 16).

The CN5-CN8 Zones interval is poorly represented at all CBT sites, except at Site 707. At deep-water sites most of the sediments are represented by redeposited material (Fig. 16). This is the interval of maximum erosion and dissolution during the Neogene in the western equatorial Indian Ocean, just as it was on a global scale (Fig. 18).

The beginning of this interval precisely correlates (Fig. 18) with a permanent increase in benthic foraminifers $\delta^{18} \mathrm{O}$ generally interpreted as related to ice buildup in Antarctica (Miller et al., 1987) and with the intensification of Antarctic Bottom Water (AABW) formation. Therefore, intensified bottom current flow and corrosiveness may have been the causal mechanisms for the shallow CCD during these times. The interval corresponds also to lowered sea-level stands and, therefore, basin- 


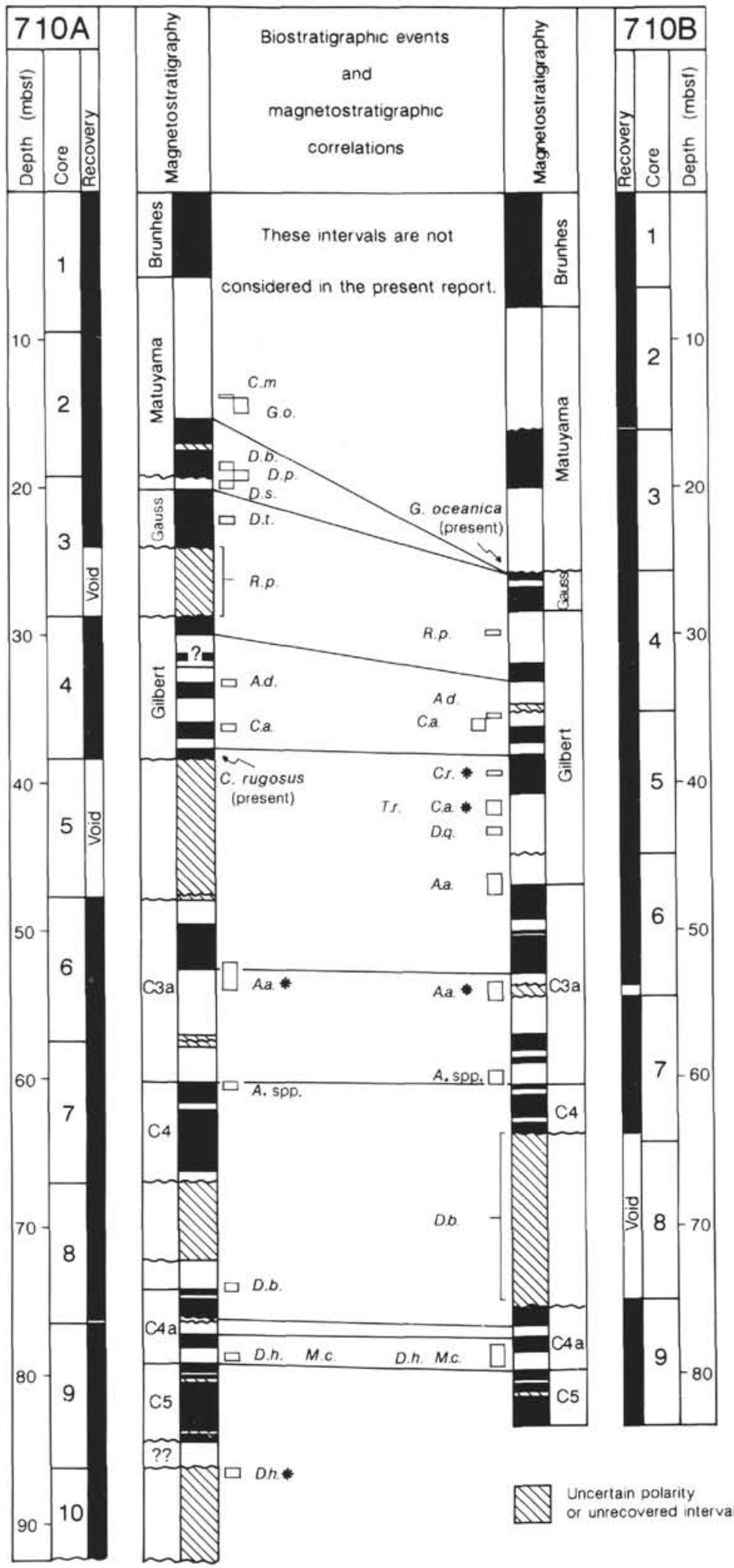

Figure 7. Interhole correlation at Site 710. Magnetostratigraphic data are from Schneider and Kent (this volume). Abbreviations for biostratigraphic events are as follows: $C . m .=C$. macintyrei $\mathrm{LO} ; G . o .=G$. oceanica $\mathrm{FO} ; D . b .=D$. brouweri LO; D.p. $=$ D. pentaradiatus LO; D.s. $=$ D. surculus LO; D.t. $=$ D. tamalis LO; R.p. $=$ R. pseudoumbilicus LO; $A . d .=A$. delicatus LO; C.a. $=$ C. acutus LO; C.r. ${ }^{*}=C$. rugosus FO; T.r. $=$ T. rugosus LO; C.a.* $=C$. acutus $\mathrm{FO} ; D . q .=D$. quinqueramus LO; $A . a .=A$. amplificus LO; $A . a .{ }^{*}=A$. amplificus $\mathrm{FO} ; A$. spp. = Amaurolithus spp. $\mathrm{FO} ; D . b .=D$. berggrenii $\mathrm{FO} ;$ $D . h .=D$. hamatus LO; M.c. $=$ M. convallis $\mathrm{FO} ; D . h^{*}=D$. hamatus $\mathrm{FO}$. 


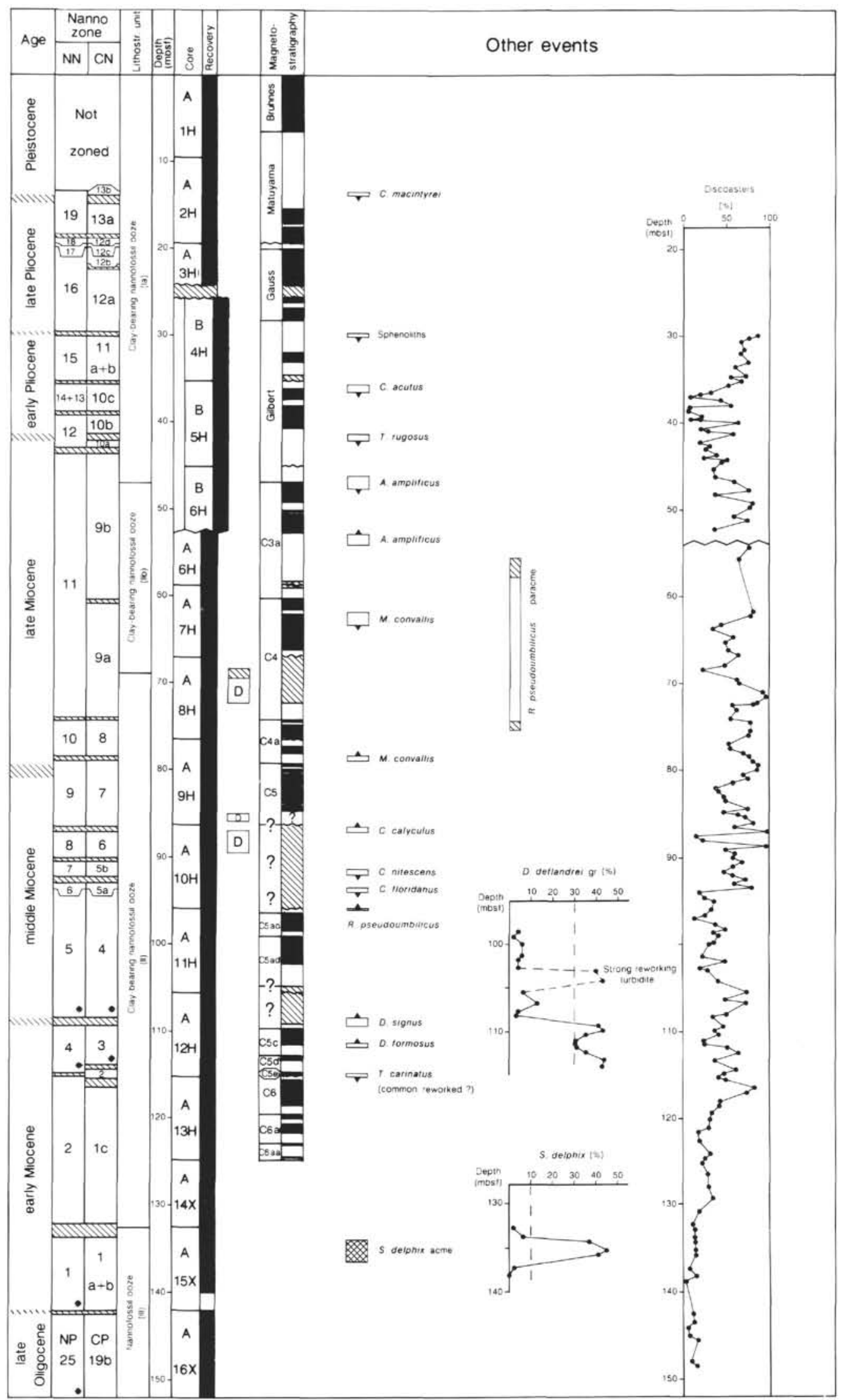

Figure 8. Chronostratigraphy and calcareous nannofossil biostratigraphy at Site 710. For legend, see Figure 3. $\mathrm{D}=$ displaced intervals. 
Table 14. Summary of the position of calcareous nannofossil events at Hole 710A.

\begin{tabular}{|c|c|c|}
\hline Event & $\begin{array}{l}\text { Core, section, } \\
\text { interval }(\mathrm{cm})\end{array}$ & $\begin{array}{l}\text { Depth } \\
\text { (mbsf) }\end{array}$ \\
\hline C. macintyrei LO & $115-710 \mathrm{~A}-2 \mathrm{H}-3,130 / 115-710 \mathrm{~A}-2 \mathrm{H}-3,90$ & $13.8-13.4$ \\
\hline G. oceanica FO & $115-710 \mathrm{~A}-2 \mathrm{H}-4,90 / 115-710 \mathrm{~A}-2 \mathrm{H}-3,130$ & 14.9-13.8 \\
\hline D. brouweri LO & $115-710 \mathrm{~A}-2 \mathrm{H}-7,30 / 115-710 \mathrm{~A}-2 \mathrm{H}-6,130$ & $18.8-18.3$ \\
\hline D. triradiatus LO & $115-710 \mathrm{~A}-2 \mathrm{H}-7,30 / 115-710 \mathrm{~A}-2 \mathrm{H}-6,130$ & $18.8-18.3$ \\
\hline D. pentaradiatus LO & $115-710 \mathrm{~A}-3 \mathrm{H}-1,30 / 115-710 \mathrm{~A}-2 \mathrm{H}-7,30$ & $19.5-18.8$ \\
\hline D. surculus LO & $115-710 \mathrm{~A}-3 \mathrm{H}-1,90 / 115-710 \mathrm{~A}-3 \mathrm{H}-1,30$ & $20.1-19.5$ \\
\hline D. tamalis LO & $115-710 \mathrm{~A}-3 \mathrm{H}-3,30 / 115-710 \mathrm{~A}-3 \mathrm{H}-2,117$ & $22.5-21.87$ \\
\hline Sphenoliths LO & $115-710 \mathrm{~A}-4 \mathrm{H}-1,30 / 115-710 \mathrm{~A}-3 \mathrm{H}-3,130$ & $29.10-23.50$ \\
\hline R. pseudoumbilicus LO & $115-710 \mathrm{~A}-4 \mathrm{H}-1,30 / 115-710 \mathrm{~A}-3 \mathrm{H}-3,130$ & $29.10-23.50$ \\
\hline Amaurolithus spp. LO & $115-710 \mathrm{~A}-4 \mathrm{H}-4,30 / 115-710 \mathrm{~A}-4 \mathrm{H}-3,130$ & $33.60-33.10$ \\
\hline C. acutus LO & $115-710 \mathrm{~A}-4 \mathrm{H}-6,30 / 115-710 \mathrm{~A}-4 \mathrm{H}-5,130$ & $36.60-36.10$ \\
\hline C. rugosus FO & $115-710 \mathrm{~A}-6 \mathrm{H}-1,130 / 115-710 \mathrm{~A}-4 \mathrm{H}-7,59$ & $49.20-38.39$ \\
\hline C. acutus FO & $115-710 \mathrm{~A}-6 \mathrm{H}-1,130 / 115-710 \mathrm{~A}-4 \mathrm{H}-\mathrm{CC}$ & $49.20-38.39$ \\
\hline T. rugosus LO & $115-710 \mathrm{~A}-6 \mathrm{H}-1,130$ & 49.20 \\
\hline D. quinqueramus LO & $115-710 \mathrm{~A}-6 \mathrm{H}-1,130$ & 49.20 \\
\hline A, amplificus LO & $115-710 \mathrm{~A}-6 \mathrm{H}-1,130$ & 49.20 \\
\hline A. amplificus $\mathrm{FO}$ & $115-710 \mathrm{~A}-6 \mathrm{H}-5,19 / 115-710 \mathrm{~A}-6 \mathrm{H}-4,1$ & $54.09-52.41$ \\
\hline Amaurolithus spp. FO & $115-710 \mathrm{~A}-7 \mathrm{H}-3,30 / 115-710 \mathrm{~A}-7 \mathrm{H}-2,130$ & $60.80-60.30$ \\
\hline M. convallis LO & $115-710 \mathrm{~A}-7 \mathrm{H}-4,130 / 115-710 \mathrm{~A}-7 \mathrm{H}-3,30$ & $63.30-61.8$ \\
\hline D. berggrenii $\mathrm{FO}$ & $115-710 \mathrm{~A}-8 \mathrm{H}-5,130 / 115-710 \mathrm{~A}-8 \mathrm{H}-5,90$ & $74.40-74.0$ \\
\hline M. convallis FO & $115-710 \mathrm{~A}-9 \mathrm{H}-2,90 / 115-710 \mathrm{~A}-9 \mathrm{H}-2,30$ & $79.0-78.4$ \\
\hline D. hamatus LO & $115-710 \mathrm{~A}-9 \mathrm{H}-2,90 / 115-710 \mathrm{~A}-9 \mathrm{H}-2,30$ & $79.0-78.4$ \\
\hline C. calyculus FO & $115-710 \mathrm{~A}-10 \mathrm{H}-1,90 / 115-710 \mathrm{~A}-10 \mathrm{H}-1,30$ & $87.10-86.5$ \\
\hline D. hamatus FO & $115-710 \mathrm{~A}-10 \mathrm{H}-1,90 / 115-710 \mathrm{~A}-10 \mathrm{H}-1,30$ & $87.10-86.5$ \\
\hline C. coalitus $\mathrm{FO}$ & $115-710 \mathrm{~A}-10 \mathrm{H}-3,130 / 115-710 \mathrm{~A}-10 \mathrm{H}-3,90$ & $90.50-90.10$ \\
\hline C. nitescens LO & $115-710 \mathrm{~A}-10 \mathrm{H}-4,130 / 115-710 \mathrm{~A}-10 \mathrm{H}-4,90$ & $92.0-91.60$ \\
\hline D. kugleri $\mathrm{FO}$ & 115-710A-10H-5, 90/115-710A-10H-5, 30 & $93.10-92.50$ \\
\hline C. floridanus LO & $115-710 \mathrm{~A}-10 \mathrm{H}-6,30 / 115-710 \mathrm{~A}-10 \mathrm{H}-5,130$ & $94.0-93.5$ \\
\hline S. heteromorphus LO & $115-710 \mathrm{~A}-10 \mathrm{H}-6,30 / 115-710 \mathrm{~A}-10 \mathrm{H}-5,130$ & $94.0-93.5$ \\
\hline R. pseudoumbilicus FO & $115-710 \mathrm{~A}-11 \mathrm{H}-1,30 / 115-710 \mathrm{~A}-10 \mathrm{H}-\mathrm{CC}$ & $96.1-95.8$ \\
\hline H. ampliaperta LO & Missing & \\
\hline D. signus FO & $115-710 \mathrm{~A}-12 \mathrm{H}-3,90 / 115-710 \mathrm{~A}-12 \mathrm{H}-2,130$ & $109.4-108.3$ \\
\hline D. deflandrei acme end & $115-710 \mathrm{~A}-12 \mathrm{H}-3,90 / 115-710 \mathrm{~A}-12 \mathrm{H}-2,130$ & $109.4-108.3$ \\
\hline D. formosus $\mathrm{FO}$ & $115-710 \mathrm{~A}-12 \mathrm{H}-5,30 / 115-710 \mathrm{~A}-12 \mathrm{H}-4,130$ & $111.8-111.3$ \\
\hline S. heteromorphus FO & $115-710 \mathrm{~A}-12 \mathrm{H}-6,130 / 115-710 \mathrm{~A}-12 \mathrm{H}-6,90$ & $114.3-113.9$ \\
\hline T. carinatus $\mathrm{LCO}$ & $115-710 \mathrm{~A}-12 \mathrm{H}-7,30 / 115-710 \mathrm{~A}-12 \mathrm{H}-6,130$ & $114.80-114.30$ \\
\hline S. belemnos LO & $115-710 \mathrm{~A}-12 \mathrm{H}-\mathrm{CC} / 115-710 \mathrm{~A}-12 \mathrm{H}-7,30$ & $115.2-114.8$ \\
\hline S. belemnos FO & $115-710 \mathrm{~A}-13 \mathrm{H}-1,130 / 115-710 \mathrm{~A}-13 \mathrm{H}-1,30$ & $116.5-115.5$ \\
\hline D. druggii FO & $115-710 \mathrm{~A}-14 \mathrm{X}-6,130 / 115-710 \mathrm{~A}-14 \mathrm{X}-5,130$ & $133.7-132.2$ \\
\hline C. abisectus $\mathrm{LO}$ & $115-710$ A-15X-5, 30/115-710A-15X-4, 130 & $138.7-138.2$ \\
\hline S. ciperoensis LO & $115-710 \mathrm{~A}-16 \mathrm{X}-1,30 / 115-710 \mathrm{~A}-15 \mathrm{X}-\mathrm{CC}$ & $142.40-142.10$ \\
\hline D. bisectus LO & $115-710 \mathrm{~A}-16 \mathrm{X}-1,130 / 115-710 \mathrm{~A}-16 \mathrm{X}-1,30$ & $143.40-142.40$ \\
\hline H. recta LO & Missing & \\
\hline Z. bijugatus LO & Poor (discontinuous occurrence) & \\
\hline
\end{tabular}

Note: $L O=$ last occurrence and $\mathrm{FO}=$ first occurrence.

Table 15. Summary of the position of calcareous nannofossil events at Hole 710B.

\begin{tabular}{|c|c|c|}
\hline Event & $\begin{array}{l}\text { Core, section, } \\
\text { interval }(\mathrm{cm})\end{array}$ & $\begin{array}{l}\text { Depth } \\
\text { (mbsf) }\end{array}$ \\
\hline G. oceanica s.1. FO & $115-710 \mathrm{~B}-4 \mathrm{H}-1,30 / 115-710 \mathrm{~B}-3 \mathrm{H}-\mathrm{CC}$ & $26.1-25.8$ \\
\hline D. brouweri 10 & $115-710 \mathrm{~B}-4 \mathrm{H}-1,30 / 115-710 \mathrm{~B}-3 \mathrm{H}-\mathrm{CC}$ & $26.1-25.8$ \\
\hline D. triradiatus LO & $115-710 \mathrm{~B}-4 \mathrm{H}-1,30 / 115-710 \mathrm{~B}-3 \mathrm{H}-\mathrm{CC}$ & $26.1-25.8$ \\
\hline D. pentaradiatus LO & $115-710 \mathrm{~B}-4 \mathrm{H}-1,30 / 115-710 \mathrm{~B}-3 \mathrm{H}-\mathrm{CC}$ & $26.1-25.8$ \\
\hline D. surculus LO & $115-710 \mathrm{~B}-4 \mathrm{H}-1,30 / 115-710 \mathrm{~B}-3 \mathrm{H}-\mathrm{CC}$ & $26.1-25.8$ \\
\hline D. tamalis LO & $115-710 \mathrm{~B}-4 \mathrm{H}-1,30 / 115-710 \mathrm{~B}-3 \mathrm{H}-\mathrm{CC}$ & $26.1-25.8$ \\
\hline Sphenoliths LO & $115-710 \mathrm{~B}-4 \mathrm{H}-3,90 / 115-710 \mathrm{~B}-4 \mathrm{H}-3,60$ & $29.7-30.1$ \\
\hline R. pseudoumbilicus LO & $115-710 \mathrm{~B}-4 \mathrm{H}-3,90 / 115-710 \mathrm{~B}-4 \mathrm{H}-3,60$ & $29.7-30.1$ \\
\hline Amaurolithus spp. LO & $115-710 \mathrm{~B}-5 \mathrm{H}-1,30 / 115-710 \mathrm{~B}-4 \mathrm{H}-\mathrm{CC}$ & $35.6-35.3$ \\
\hline C. acutus LO & $115-710 \mathrm{~B}-5 \mathrm{H}-1,120 / 115-710 \mathrm{~B}-5 \mathrm{H}-1,30$ & $36.5-35.6$ \\
\hline C. rugosus $\mathrm{FO}$ & $115-710 \mathrm{~B}-5 \mathrm{H}-3,120 / 115-710 \mathrm{~B}-5 \mathrm{H}-3,90$ & $39.5-39.2$ \\
\hline C. acutus FO & $115-710$ B- $5 \mathrm{H}-5,90 / 115-710 \mathrm{~B}-5 \mathrm{H}-4,150$ & $42.2-41.3$ \\
\hline T. rugosus LO & 115-710B-5H-5, 90/115-710B-5H-4, 150 & $42.2-41.3$ \\
\hline D. quinqueramus LO & 115-710B-5H-6, 90/115-710B-5H-6, 30 & $43.7-43.1$ \\
\hline A. amplificus LO & $115-710 \mathrm{~B}-6 \mathrm{H}-2,130 / 115-710 \mathrm{~B}-6 \mathrm{H}-1,130$ & $47.7-46.2$ \\
\hline A. amplificus $\mathrm{FO}$ & $115-710 \mathrm{~B}-7 \mathrm{H}-1,30 / 115-710 \mathrm{~B}-6 \mathrm{H}-6,110$ & $54.8-53.5$ \\
\hline Amaurolithus spp. FO & 115-710B-7H-5, 30/115-710B-7H-4, 130 & $60.8-60.3$ \\
\hline D. berggrenii $\mathrm{FO}$ & Within void core 8 & \\
\hline M. convallis $\mathrm{FO}$ & $115-710 \mathrm{~B}-9 \mathrm{H}-4,120 / 115-710 \mathrm{~B}-9 \mathrm{H}-3,120$ & $79.5-78.0$ \\
\hline D. hamatus LO & $115-710 \mathrm{~B}-9 \mathrm{H}-4,120 / 115-710 \mathrm{~B}-9 \mathrm{H}-3,120$ & $79.5-78.0$ \\
\hline
\end{tabular}

Note: $\mathrm{LO}=$ last occurrence and FO = first occurrence. shelf organic carbon fractionation (Broecker, 1981, 1982) may also have been effective in causing this deep-basin carbonate crisis.

This interval is also characterized by hiatuses at Site 709 and by redeposition in deep-water Sites 708 and 710 (Fig. 16). Slope instability, related to glacial build-up or tectonic causes, may have been active.

\section{Late Miocene Carbonate Optimum}

The shallow CCD of the middle Miocene ended at the CN8/ CN9 boundary, when very dissolved coccolith assemblages reenter the deep water Site 711 (Fig. 16). Concomitant with this change, discoasterid abundance decreases at Site 710 (Fig. 8). This change occurs at the top of Chron C4A (Fig. 14) -that is, at about $8 \mathrm{Ma}$-and marks the beginning of an interval of high sedimentation of carbonate that will last up to the base of the Gilbert Chron, at about 5-5.3 Ma. An increase in the carbonate accumulation is noted at all sites, and hiatuses are not observed within the limits of current biostratigraphic resolution (Fig. 16). Within this 3-m.y.-long interval sediment thicknesses are about $30 \mathrm{~m}$ at Site 707 , about $45 \mathrm{~m}$ at Site 709 , and about $17-18 \mathrm{~m}$ at Site 711 . At Sites 708 and 710 , the record is characterized by turbidites and redeposition (Fig. 16).

The beginning of this interval correlates fairly well with a further shift in the $\delta^{18} \mathrm{O}$ of benthic foraminifers, a general climatic deterioration, and an increase in carbonate contents in the equatorial Pacific Ocean sediments (Fig. 18). A rapid deepening of the CCD in the late Miocene is a phenomenon also recorded in the Atlantic Ocean, where it was interpreted as being caused by the production of North Atlantic Deep Water (NADW), which actively erodes the AABW and thus diminishes its oceanographic influence on the chemistry and fertility of the Atlantic (Berger, 1978).

Most probably oceanographic reorganization also occurred in the western equatorial Indian Ocean, which changed fertility patterns with increased carbonate bioproduction. No major response appears to be present in our record which can be linked to the isolation of the Mediterranean (Messinian Event), about between 6.5 and $5.0 \mathrm{Ma}$ (Fig. 18).

\section{Early Pliocene Shoaling of the CCD}

Approximately from the early Gilbert Chron to the middle of the Gauss Chron, nannofossils virtually disappear again, or are represented almost solely by discoasterids, at Site 711 (Figs. 14 and 17), thus indicating a further shoaling of the CCD. No obvious major change is correlatable with this interval, the base of which correlates with a decrease in carbonate contents in the equatorial Pacific sediments and with the reestablishment of open-marine conditions in the Mediterranean (Fig. 18). No hiatus is detectable with nannofossil biostratigraphy in this interval (Fig. 17).

\section{Mid-Pliocene Deepening of the CCD}

Calcareous nannofossils reenter the Site 711 record at about 3.0 Ma (Fig. 14) and are present throughout the late Pliocene and Pleistocene. This seems to be the time of the deepening of the CCD to the present-day deep values. This change corresponds with a climatic deterioration (Prell, 1984; Keigwin, 1982, 1987), which slightly preceded the onset of the Northern Hemisphere glaciation at 2.5 Ma (Shackleton et al., 1984).

Sedimentation appears continuous at Sites 707, 710, and 711 (Fig. 16). Note in Figure 16 the expanded sequence at deep-water Site 708, which is caused by the strong redeposition of turbidity currents. Hiatuses are present close to the Pliocene/Pleistocene boundary at Site 709 , and in the late Pliocene and early Pleistocene at Site 710. 


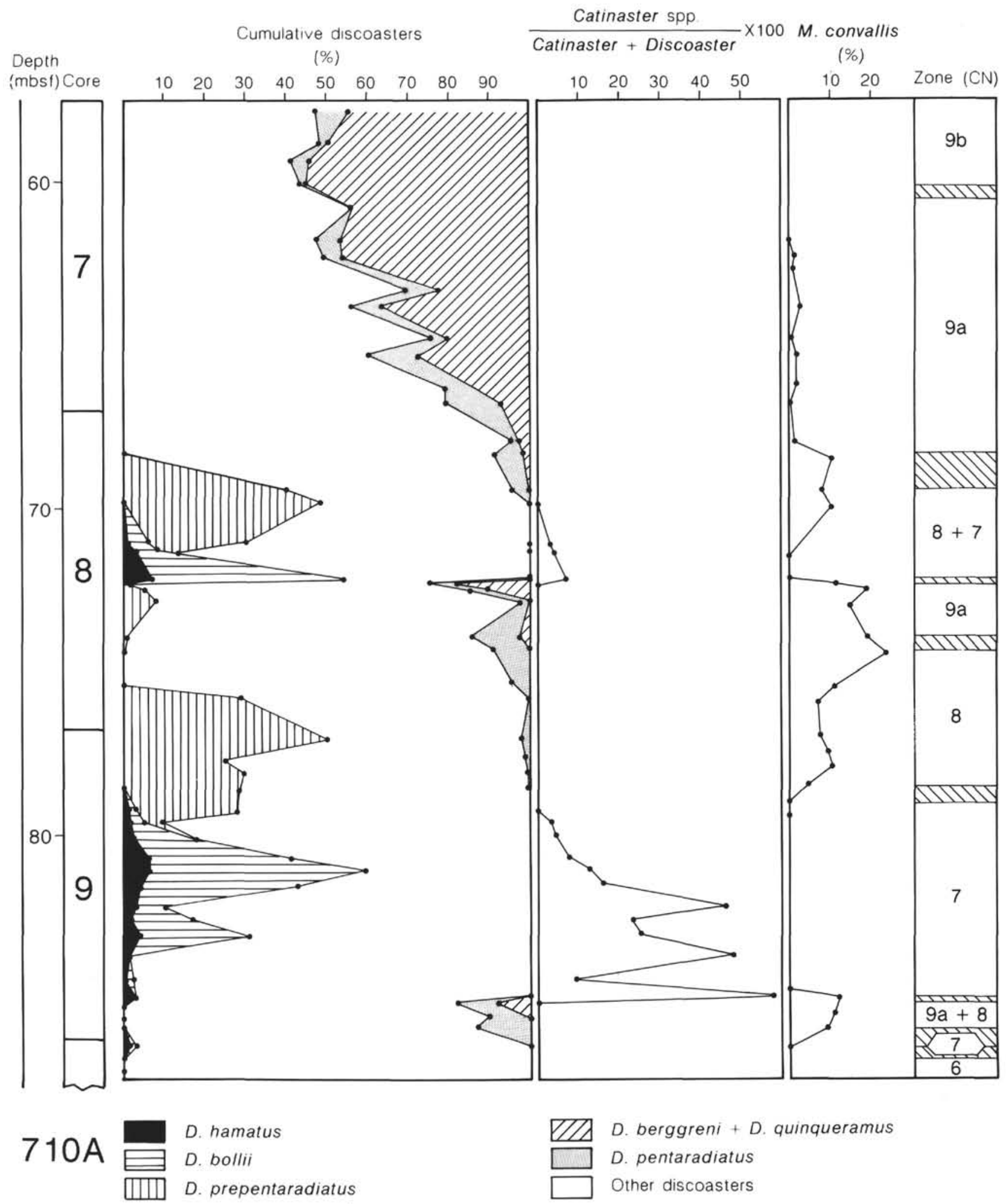

Figure 9. Plots of abundance patterns of discoasterids, Catinaster spp., and Minylitha convallis at Hole 710A showing stratigraphic inversions during late Miocene time. Discoasterid cumulative percentages and the abundance of Catinaster spp. are based on a count of about 300 specimens per sample. $M$. convallis abundance is based on a count of about 500 nannofossils. 


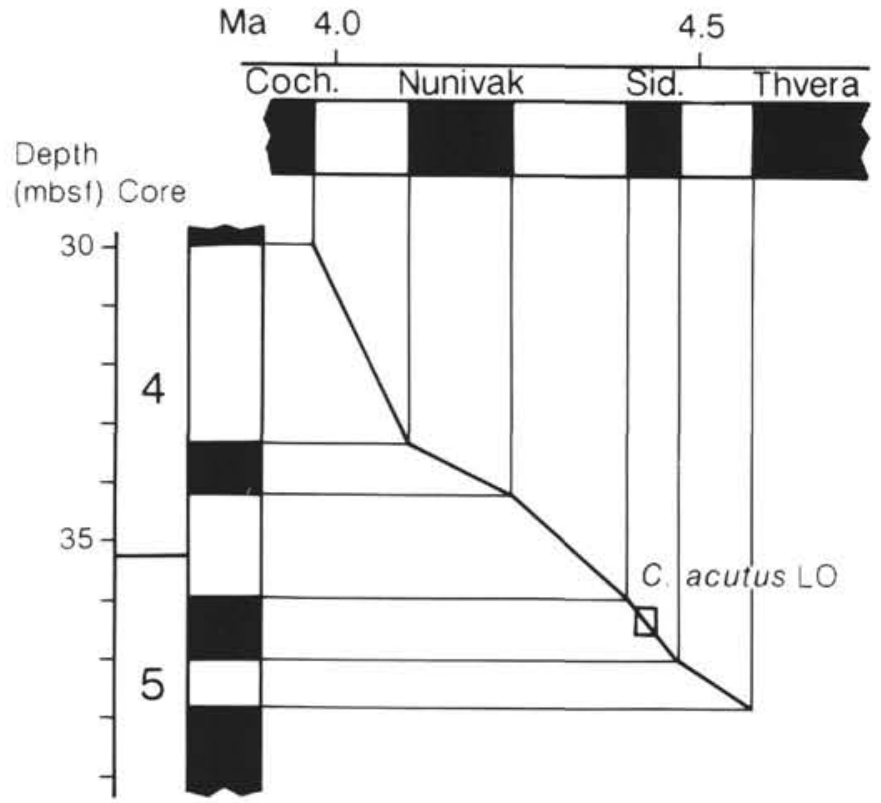

Figure 10. Age-depth plot of early Pliocene sediments at Hole 710A based on magnetostratigraphic data of Schneider and Kent (this volume).

\section{Chagos Sites \\ (Sites 712 and 713)}

Sites 712 and 713 were drilled only $1.6 \mathrm{nmi}$ apart on the northern margin of the Chagos Bank (Fig. 1 and Table 1). A single hole was rotary drilled at Site 712 , which ended in middle Eocene nannofossil ooze and volcanic ashes at $115.3 \mathrm{mbsf}$. At Site 713 a single hole was rotary drilled, which ended in basalt flows intercalated with nannofossil chalk of early middle Eocene age, at 192 mbsf. Pliocene and Miocene sediments were retrieved at both sites. Calcareous nannofossils are abundant and moderately preserved, and their stratigraphic distribution is presented in Tables 21 and 22 (microfiche, back pocket). Reworking is strong at both sites (Tables 21 and 22). The events detected are summarized in Tables 23 and 24 . The stratigraphic sequences retrieved at these nearby sites are intercorrelated in Figure 19, where some remarkable differences are noteworthy.

The latest Pliocene and Pleistocene sediments are missing at Site 712 , whereas they are present at Site 713 . In the early Pliocene, sedimentation rates were higher at Site 712. At both sites the FO of Amaurolithus spp. (late Miocene) occurs above a discontinuity, marking the end of an erosional event that was much more intense at Site 713, where it removed Miocene, Oligocene, and late Eocene sediments. At Site 712 the erosional event removed only late Miocene sediments of Subzone CN9a. It is to be noted that a change in preservation of calcareous nannofossils is present at Site 712 between sediments of Subzone CN9b

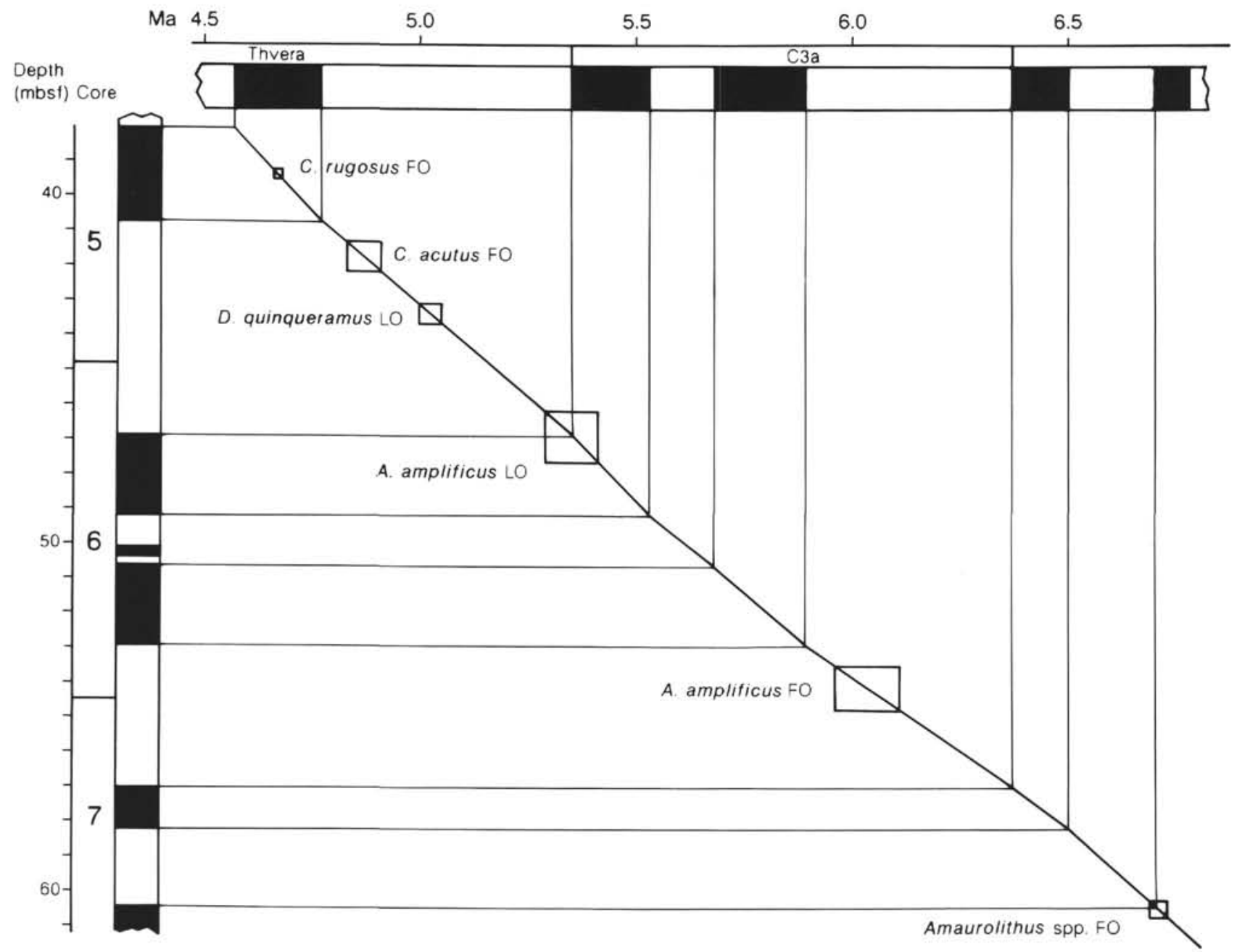

Figure 11. Age-depth plot of late Miocene-early Pliocene sediments at Hole 710B based on magnetostratigraphic data of Schneider and Kent (this volume). 


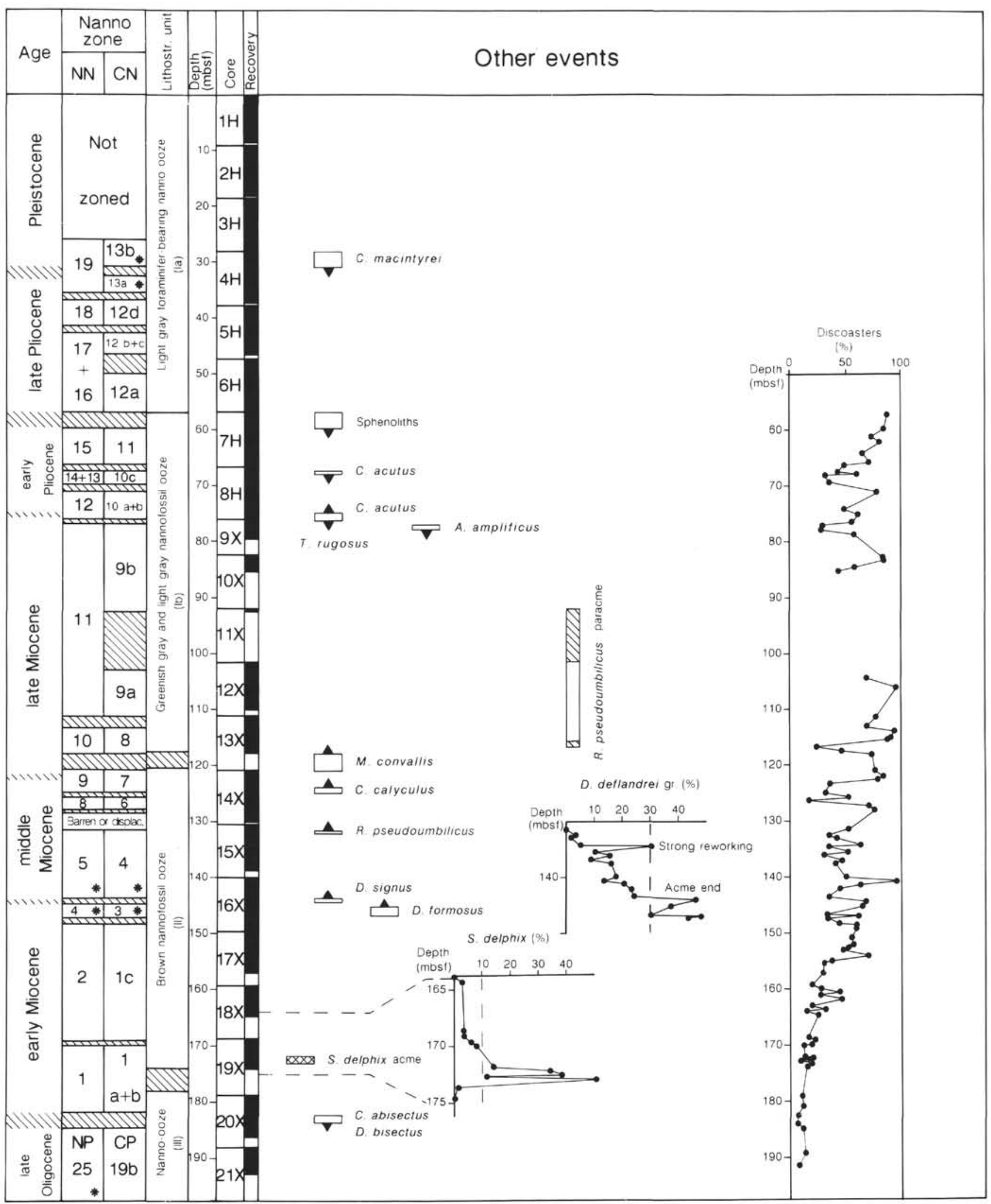

Figure 12. Chronostratigraphy and calcareous nannofossil biostratigraphy at Site 708. For legend, see Figure 3. 
Table 17. Summary of the position of calcareous nannofossil events at Hole 708A.

\begin{tabular}{|c|c|c|}
\hline Event & $\begin{array}{l}\text { Core, section, } \\
\text { interval }(\mathrm{cm})\end{array}$ & $\begin{array}{l}\text { Depth } \\
\text { (mbsf) }\end{array}$ \\
\hline C. macintyrei LO & $115-708 \mathrm{~A}-4 \mathrm{H}-2,130 / 115-708 \mathrm{~A}-3 \mathrm{H}-\mathrm{CC}$ & $30.90-28.1$ \\
\hline G. oceanica FO & $115-708 \mathrm{~A}-4 \mathrm{H}-3,130 / 115-708 \mathrm{~A}-4 \mathrm{H}-2,130$ & $32.40-30.90$ \\
\hline D. brouweri LO & $115-708 \mathrm{~A}-4 \mathrm{H}-6,130 / 115-708 \mathrm{~A}-4 \mathrm{H}-5,130$ & $36.90-35.40$ \\
\hline D. triradiatus LO & $115-708 \mathrm{~A}-4 \mathrm{H}-6,130 / 115-708 \mathrm{~A}-4 \mathrm{H}-5,130$ & $36.90-35.40$ \\
\hline D. pentaradiatus $\mathrm{LO}$ & $115-708 \mathrm{~A}-5 \mathrm{H}-4,55 / 115-708 \mathrm{~A}-5 \mathrm{H}-3,80$ & $42.65-41.40$ \\
\hline D. surculus LO & $115-708 \mathrm{~A}-5 \mathrm{H}-4,55 / 115-708 \mathrm{~A}-5 \mathrm{H}-3,80$ & $42.65-41.40$ \\
\hline D. tamalis LO & $115-708 \mathrm{~A}-6 \mathrm{H}-2,130 / 115-708 \mathrm{~A}-5 \mathrm{H}-6,120$ & $50.0-46.30$ \\
\hline Sphenoliths LO & $115-708 \mathrm{~A}-7 \mathrm{H}-2,130 / 115-708 \mathrm{~A}-6 \mathrm{H}-\mathrm{CC}$ & $59.60-56.8$ \\
\hline R. pseudoumbilicus LO & $115-708 \mathrm{~A}-7 \mathrm{H}-2,130 / 115-708 \mathrm{~A}-6 \mathrm{H}-\mathrm{CC}$ & $59.60-56.8$ \\
\hline Amaurolithus spp. LO & $115-708 \mathrm{~A}-8 \mathrm{H}-1,100 / 115-708 \mathrm{~A}-7 \mathrm{H}-7,53$ & $67.40-66.33$ \\
\hline C. acutus LO & $115-708 \mathrm{~A}-8 \mathrm{H}-2,18 / 115-708 \mathrm{~A}-8 \mathrm{H}-1,130$ & $68.08-67.70$ \\
\hline C. rugosus FO & $115-708 \mathrm{~A}-8 \mathrm{H}-4,20 / 115-708 \mathrm{~A}-8 \mathrm{H}-3,40$ & $71.10-69.80$ \\
\hline C. acutus $\mathrm{FO}$ & $115-708 \mathrm{~A}-9 \mathrm{X}-1,30 / 115-708 \mathrm{~A}-8 \mathrm{H}-6,100$ & $76.30-74.90$ \\
\hline T. rugosus LO & $115-708 \mathrm{~A}-9 \mathrm{X}-1,30 / 115-708 \mathrm{~A}-8 \mathrm{H}-6,100$ & $76.30-74.90$ \\
\hline D. quinqueramus LO & 115-708A-9X-1, 82/115-708A-9X-1, 30 & $76.82-76.30$ \\
\hline A. amplificus LO & 115-708A-9X-2, 30/115-708A-9X-1, 82 & $77.80-76.82$ \\
\hline A. amplificus $\mathrm{FO}$ & $115-708 \mathrm{~A}-10 \mathrm{X}-\mathrm{CC} / 115-708 \mathrm{~A}-10 \mathrm{X}-2,30$ & $91.70-84.90$ \\
\hline Amaurolithus spp. FO & $115-708 \mathrm{~A}-12 \mathrm{X}-2,130 / 115-708 \mathrm{~A}-11 \mathrm{X}-\mathrm{CC}$ & $104.20-101.4$ \\
\hline D. berggrenii $\mathrm{FO}$ & $115-708 \mathrm{~A}-13 \mathrm{X}-2,86 / 115-708$ & $113.36-111.25$ \\
\hline M. convallis $\mathrm{FO}$ & $115-708 \mathrm{~A}-14 \mathrm{X}-1,40 / 115-708 \mathrm{~A}-13 \mathrm{X}-\mathrm{CC}$ & $121.0-120.6$ \\
\hline D. hamatus LO & $115-708 \mathrm{~A}-13 \mathrm{X}-\mathrm{CC} / 115-708 \mathrm{~A}-13 \mathrm{X}-5,100$ & $120.6-118.0$ \\
\hline C. calyculus FO & $-2,130$ & $124.9-123.9$ \\
\hline D. hamatus FO & $115-708 \mathrm{~A}-14 \mathrm{X}-4,67 / 115-708 \mathrm{~A}-1$ & $125.77-124.90$ \\
\hline C. coalitus FO & $115-708 \mathrm{~A}-14 \mathrm{X}-5,130$ & 127.90 \\
\hline C. nitescens LO & ND & \\
\hline D. kugleri $\mathrm{FO}$ & ND & \\
\hline C. floridanus $\mathrm{LO}$ & $115-7$ & 131.60 \\
\hline S. heteromorphus LO & $115-708 \mathrm{~A}-15 \mathrm{X}-1,130$ & 131.60 \\
\hline R. pseudoumbilicus FO & $115-708 \mathrm{~A}-15 \mathrm{X}-2,73 / 115-708 \mathrm{~A}-15 \mathrm{X}-1,130$ & $132.53-131.60$ \\
\hline H. & Missing & \\
\hline D. signus $\mathrm{FO}$ & $115-708 \mathrm{~A}-16 \mathrm{X}-3,130 / 115-708 \mathrm{~A}-1$ & 144.30 \\
\hline D. deflandrei acme end & $115-708 \mathrm{~A}-16 \mathrm{X}-3,130 / 115-708 \mathrm{~A}-16 \mathrm{X}-3,62$ & $144.30-143.62$ \\
\hline D. formosus FO & $115-708 \mathrm{~A}-16 \mathrm{X}-5,90 / 115-708 \mathrm{~A}-16 \mathrm{X}-4,70$ & $146.90-145.20$ \\
\hline S. heteromorphus FO & A-16X-6, 91/115-708A-16X-5, 130 & $148.41-147.3$ \\
\hline S. belemnos LO & $-16 X-6,91 / 115-708 \mathrm{~A}-16 \mathrm{X}-5,130$ & $148.41-147.3$ \\
\hline S. belemnos FO & $115-708 \mathrm{~A}-16 \mathrm{X}-6,91 / 115-708 \mathrm{~A}-16 \mathrm{X}-5,130$ & $148.41-147.3$ \\
\hline$T$. carinatus LO & ND (strong reworking) & \\
\hline D. druggii $\mathrm{FO}$ & $115-708 \mathrm{~A}-19 \mathrm{X}-1,119 / 115$ & 169.79 \\
\hline sis Lo & $-3,107 / 115-708 \mathrm{~A}-$ & $182.27-180.82$ \\
\hline C. abisectus LO & $115-708 \mathrm{~A}-20 \mathrm{X}-4,112 / 115-708 \mathrm{~A}-20 \mathrm{X}-3,107$ & $183.82-182.27$ \\
\hline D. bisectus LO & $115-708 \mathrm{~A}-20 \mathrm{X}-4,112 / 115-708 \mathrm{~A}-20 \mathrm{X}-3,107$ & $183.82-182.27$ \\
\hline H. recta $\mathrm{LO}$ & Missing & \\
\hline Z. bijugatus LO & ND & \\
\hline
\end{tabular}

Note: $\mathrm{LO}=$ last occurrence, $\mathrm{FO}=$ first occurrence, and ND $=$ not detectable.

Table 18. Summary of the position of calcareous nannofossil events at Hole 711A.

\begin{tabular}{|c|c|c|}
\hline Event & $\begin{array}{l}\text { Core, section, } \\
\text { interval }(\mathrm{cm})\end{array}$ & $\begin{array}{l}\text { Depth } \\
\text { (mbsf) }\end{array}$ \\
\hline G. oceanica FO & $115-711 \mathrm{~A}-2 \mathrm{H}-1,138 / 115-711 \mathrm{~A}-2 \mathrm{H}-1,70$ & $9.48-8.8$ \\
\hline D. brouweri LO & $115-711 \mathrm{~A}-2 \mathrm{H}-1,138 / 115-711 \mathrm{~A}-2 \mathrm{H}-1,70$ & $9.48-8.8$ \\
\hline D. pentaradiatus $\mathrm{LO}$ & $115-711 \mathrm{~A}-2 \mathrm{H}-3,138 / 115-711 \mathrm{~A}-2 \mathrm{H}-3,70$ & $12.48-11.8$ \\
\hline D. surculus LO & ND & \\
\hline D. tamalis LO & ND & \\
\hline C. rugosus $\mathrm{FO}$ & $115-711 \mathrm{~A}-3 \mathrm{H}-2,60$ & 19.8 \\
\hline C. acutus LO & $115-711 \mathrm{~A}-3 \mathrm{H}-2,60$ & 19.8 \\
\hline D. berggrenii LO & $115-711 \mathrm{~A}-3 \mathrm{H}-4,115$ & 23.35 \\
\hline Amaurolithus spp. FO & $115-711 \mathrm{~A}-4 \mathrm{H}-4,130 / 115-711 \mathrm{~A}-4 \mathrm{H}-3,130$ & $33.1-31.6$ \\
\hline D. berggrenii $\mathrm{FO}$ & 115-711A-5H-1, 90/115-711A-5H-1, 60 & $37.8-37.5$ \\
\hline S. heteromorphus LO & $115-711 \mathrm{~A}-6 \mathrm{H}-2,80$ & 48.9 \\
\hline S. heteromorphus FO & $115-711 \mathrm{~A}-7 \mathrm{H}-1,60$ & 56.9 \\
\hline T. carinatus LO & $115-711 \mathrm{~A}-7 \mathrm{H}-6,60$ & 64.4 \\
\hline D. druggii FO & $115-711 \mathrm{~A}-8 \mathrm{H}-\mathrm{CC}$ & 75.6 \\
\hline S. ciperoensis LO & $115-711 \mathrm{~A}-9 \mathrm{H}-2,130 / 115-711 \mathrm{~A}-9 \mathrm{H}-2,85$ & $78.40-77.95$ \\
\hline
\end{tabular}

Note: $\mathrm{LO}=$ last occurrence, $\mathrm{FO}=$ first occurrence, and ND $=$ not detected

and the underlying sediments of Zone CN8. At Site 712 a compressed interval of middle Miocene sediments was recovered (Fig. 19 and Table 21) that rests on a poorly recovered Oligocene sequence (Backman, Duncan, et al., 1988).
Table 19. Summary of the position of calcareous nannofossil events at Hole $711 B$.

\begin{tabular}{|c|c|c|}
\hline Event & $\begin{array}{l}\text { Core, section, } \\
\text { interval }(\mathrm{cm})\end{array}$ & $\begin{array}{l}\text { Depth } \\
\text { (mbsf) }\end{array}$ \\
\hline C. macintyrei LO & $115-711 \mathrm{~B}-2 \mathrm{H}-4,125 / 115-711 \mathrm{~B}-2 \mathrm{H}-4,100$ & $7.85-7.60$ \\
\hline G. oceanica s.I. FO & 115-711B-2H-5, 50/115-711B-2H-5, 25 & $8.60-8.35$ \\
\hline D. brouweri LO & $115-711 \mathrm{~B}-2 \mathrm{H}-6,25 / 115-711 \mathrm{~B}-2 \mathrm{H}-5,150$ & $9.85-9.60$ \\
\hline D. triradiatus LO & $115-711 \mathrm{~B}-2 \mathrm{H}-6,25 / 115-711 \mathrm{~B}-2 \mathrm{H}-5,150$ & $9.85-9.60$ \\
\hline D. pentaradiatus $\mathrm{LO}$ & $115-711 \mathrm{~B}-2 \mathrm{H}-\mathrm{CC} / 115-711 \mathrm{~B}-2 \mathrm{H}-7,45$ & $11.70-11.55$ \\
\hline D. surculus LO & $115-711 \mathrm{~B}-3 \mathrm{H}-1,75 / 115-711 \mathrm{~B}-3 \mathrm{H}-1,150$ & $12.45-12.20$ \\
\hline D. tamalis LO & ND & \\
\hline Sphenoliths LO & $115-711 \mathrm{~B}-3 \mathrm{H}-5,75 / 115-711 \mathrm{~B}-3 \mathrm{H}-2,25$ & $18.45-13.45$ \\
\hline R. pseudoumbilicus LO & $115-711 \mathrm{~B}-3 \mathrm{H}-5,75 / 115-711 \mathrm{~B}-3 \mathrm{H}-2,25$ & $18.45-13.45$ \\
\hline Amaurolithus spp. LO & $115-711$ B- $3 \mathrm{H}-5,75 / 115-711 \mathrm{~B}-3 \mathrm{H}-2,25$ & $18.45-13.45$ \\
\hline C. acutus LO & 115-711B-3H-5, 75/ & 13.45 \\
\hline C. rugosus FO & B- $3 \mathrm{H}-5,125$ & $19.20-18.95$ \\
\hline C. acutus $\mathrm{FO}$ & $115-711 \mathrm{~B}-3 \mathrm{H}-5,150 / 115-711 \mathrm{~B}-3 \mathrm{H}-5,125$ & $19.20-18.95$ \\
\hline T. rugosus LO & $115-711 \mathrm{~B}-4 \mathrm{H}-1,25$ & 21.65 \\
\hline D. quinqueramus LO & $115-711 \mathrm{~B}-4 \mathrm{H}-1,25$ & 21.65 \\
\hline A. amplificus LO & $115-711 \mathrm{~B}-4 \mathrm{H}-1,100 / 115-711 \mathrm{~B}-4 \mathrm{H}-1,75$ & $22.40-22.15$ \\
\hline A. amplificus $\mathrm{FO}$ & $115-711 \mathrm{~B}-4 \mathrm{H}-5,25 / 115-711$ & $27.65-27.40$ \\
\hline Amaurolithus spp. FO & $115-711 \mathrm{~B}-4 \mathrm{H}-7,10 / 115-711 \mathrm{~B}-4 \mathrm{H}-6,150$ & $30.50-30.40$ \\
\hline D. berggrenii $\mathrm{FO}$ & $\mathrm{H}-4,145 / 115-711 \mathrm{~B}-5 \mathrm{H}-4,125$ & $36.95-36.75$ \\
\hline M. convallis $\mathrm{FO}$ & $115-711 \mathrm{~B}-5 \mathrm{H}-5,20$ & 37.20 \\
\hline S. heteromorphus LO & $115-711 \mathrm{~B}-6 \mathrm{H}-4,94$ & 46.14 \\
\hline R. pseudoumbilicus FO & $115-711$ B- $6 \mathrm{H}-4,125 / 115-711 \mathrm{~B}-6 \mathrm{H}-4,100$ & $46.2-46.45$ \\
\hline H. ampliaperta LO & Missing & \\
\hline D. signus FO & $115-711 \mathrm{~B}-7 \mathrm{H}-2,10$ & $52.89-52.30$ \\
\hline D. deflandrei acme end & $115-711 \mathrm{~B}-7 \mathrm{H}-2,109 / 115-711 \mathrm{~B}-7 \mathrm{H}-2,50$ & $52.89-52.30$ \\
\hline D. formosus $\mathrm{FO}$ & $115-711 \mathrm{~B}-7 \mathrm{H}-3,41 / 115-711 \mathrm{~B}-7 \mathrm{H}-3,17$ & $53.71-53.47$ \\
\hline S. heteromorphus FO & $115-711 \mathrm{~B}-7 \mathrm{H}-3,70$ & 54.0 \\
\hline T. carinatus LO & $115-711 \mathrm{~B}-8 \mathrm{H}-1,106$ & 61.06 \\
\hline D. druggii FO & $115-711 \mathrm{~B}-8 \mathrm{H}-6,150 / 115-711 \mathrm{~B}-8 \mathrm{H}-6,50$ & $69.0-68.0$ \\
\hline C. abisectus LC & ND (strong reworking) & \\
\hline S. ciperoensis LO & 115-711B-10H-1, 50/115-711B-9H-CC & $79.80-79.30$ \\
\hline D. bisectus LO & ND (strong reworking) & \\
\hline H. recta $\mathrm{LO}$ & Missing & \\
\hline Z. bijugatus LO & ND (strong reworking) & \\
\hline
\end{tabular}

Note: $\mathrm{LO}=$ last occurrence, $\mathrm{FO}=$ first occurrence, and $\mathrm{ND}=$ not detectable.

\section{Maldives Sites \\ (Sites 714, 715, and 716)}

\section{Site 714}

Two holes were continuously cored at Site 714. Hole 714A ended at 233.0 mbsf, in foraminifer-nannofossil chalk, late Oligocene in age. Hole 714B ended at $122.6 \mathrm{mbsf}$, in a foraminiferbearing nannofossil ooze, at approximately the early/middle Miocene boundary. The Neogene sequence at this site contains a megahiatus between late Pleistocene and early late Miocene sediments (Fig. 20). Calcareous nannofossils are abundant in the Miocene sediments with a preservation state that varies from good to moderate. Reworking is minimal. Etching is minimal at this shallow-water site, although overgrowth varies and is strong in some intervals. Cyclic changes in color occur in Zones NN6$\mathrm{NN} 10$, that seem to correlate with the preservation of discoasterids.

For detailed shore-based studies we have concentrated on Hole 714A (Fig. 20 and Tables 25 [microfiche, back pocket] and 26). At this hole recovery was poor in Core $115-714 \mathrm{~A}-9 \mathrm{H}$ at the transition between Zones NN6 and NN7. This interval was well recovered at Hole 714B. Therefore, we have determined biostratigraphic events at this hole as well, which are summarized in Table 27.

The calcareous nannofossil assemblages show a high taxonomic diversity at this site in comparison with other Leg 115 sites. Helicoliths, scyphospherids, pontospherids and Thoracosphaera spp., and holococcoliths are consistently present at Site 714 , but are missing or show discontinuous occurrences at other Leg 115 sites. This feature can only be partially explained by minor dissolution at this shallow-water site; most probably it is 


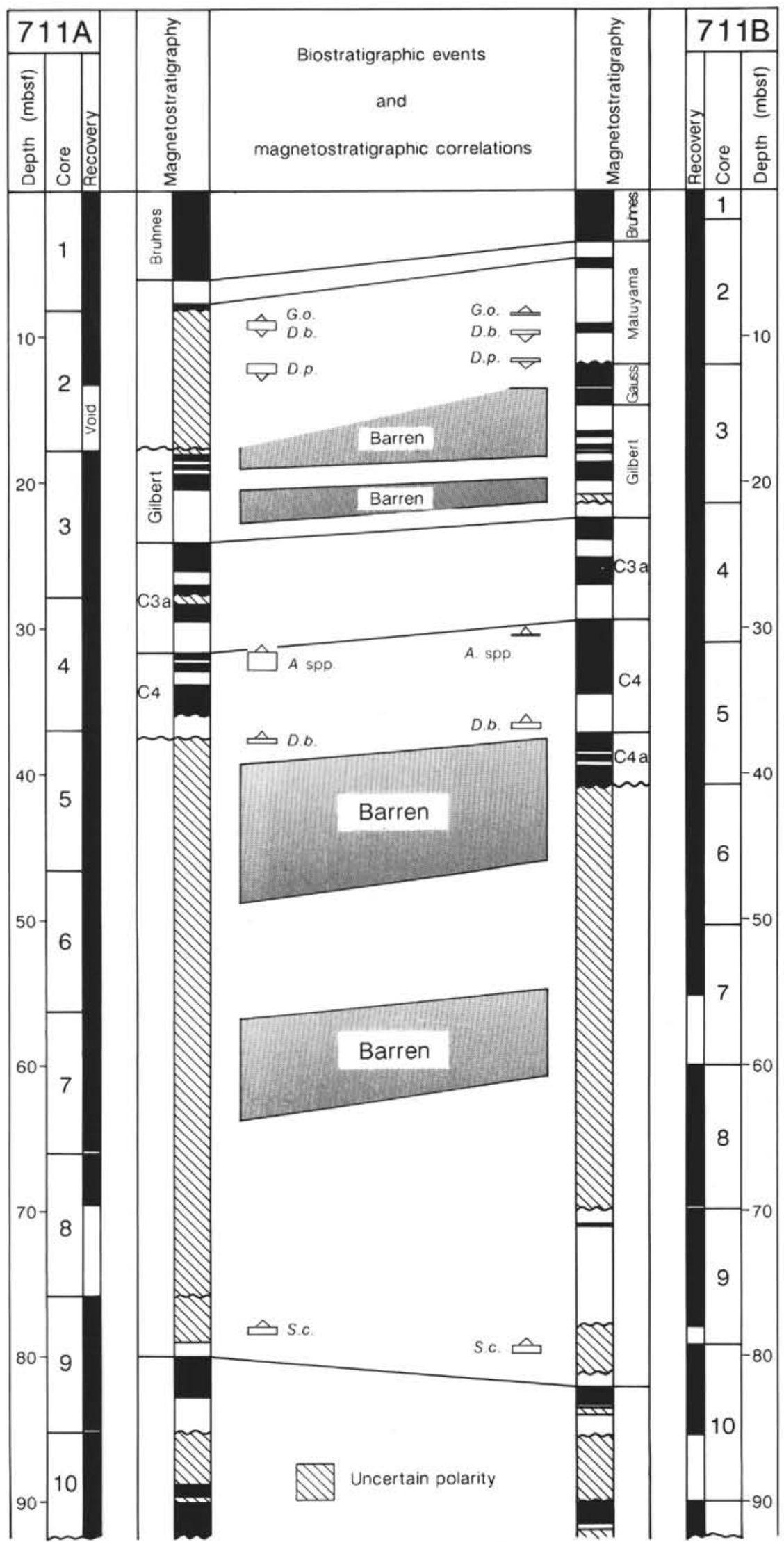

Figure 13. Interhole correlation at Site 711. Magnetostratigraphic data are after Schneider and Kent (this volume). 


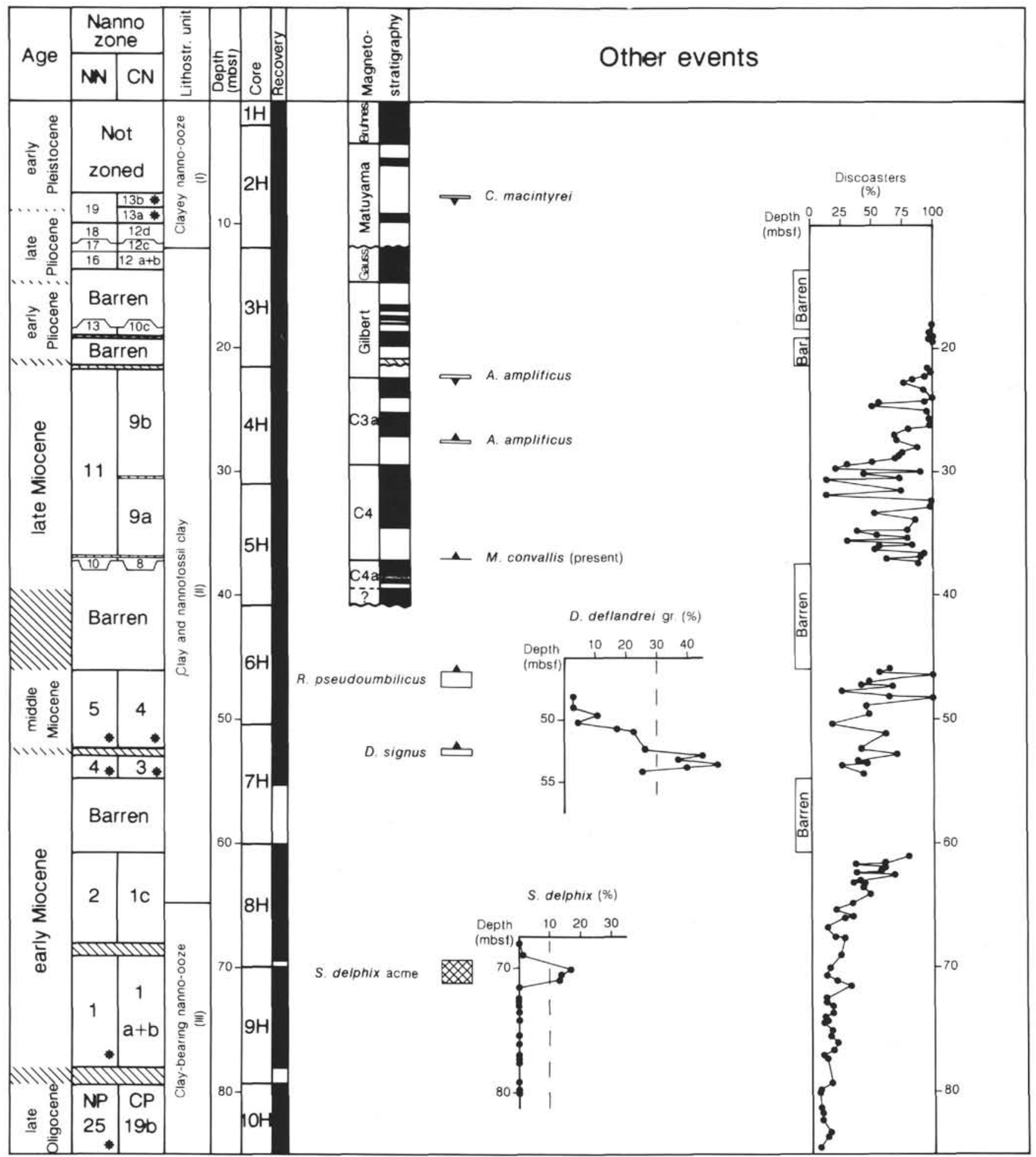

Figure 14. Chronostratigraphy and calcareous nannofossil biostratigraphy at Site 711. Magnetostratigraphic data are after Schneider and Kent (this volume). For legend, see Figure 3.

primarily related to influence of different water masses with respect to the Mascarene CBT sites.

The consistent presence of helicoliths is biostratigraphically significant in so far as it allows us to compare the distributions of $H$. recta and $H$. ampliaperta (primary zonal markers in the standard zonations that are often missing in oceanic sediments) with other alternative events (see Fig. 20).
Site 715

Site 715 is located on the eastern margin of the Maldives Ridge, in $2273 \mathrm{~m}$ water depth, only $3 \mathrm{nmi}$ to the west of Site 714. A single hole was rotary drilled at Site 715. The main objective was to penetrate the basement, which was reached at 211 mbsf. Above the basalt was $100 \mathrm{~m}$ of Paleogene shallow-wa- 


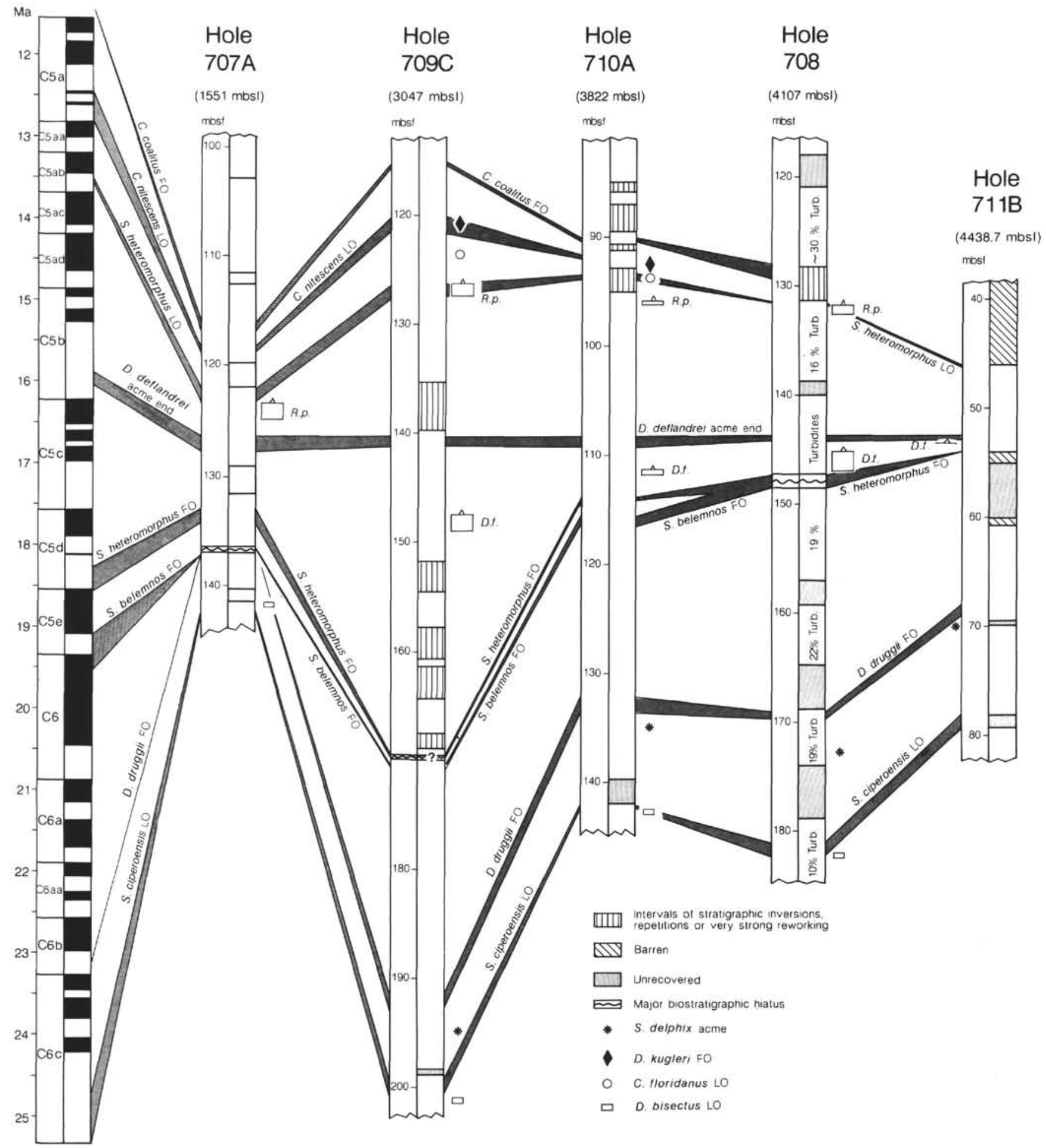

Figure 15. Early-middle Miocene correlations among CBT sites. Major stratigraphic features and main biostratigraphic events are shown. R.p. = Reticulofenestra pseudoumbilicus; D.f. = Discoaster formosus.

ter limestones overlain by some $100 \mathrm{~m}$ of Neogene nannofossil ooze. As at Site 714, a megahiatus is present (Core 115-715A-6R) (Fig. 21) between Pleistocene and middle Miocene nannofossil oozes.

Data collected in the Miocene sequence at this site are summarized in Figure 21 and in Tables 28 (microfiche, back pocket) and 29. Calcareous nannofossils are abundant and well diversified throughout the sequence, as at Site 714. Preservation is from good to moderate, discoasters being generally overgrown. Biostratigraphic assignments (Fig. 21) are straightforward, except for the lower part of Core 115-715A-11R, below the FO of $S$. belemnos. In this interval $D$. druggii is missing, and it is not clear whether this absence is caused by ecologic control and too sparse sampling or if a hiatus is present that removed Zone NN2 (CN1c). Sediments below Core 115-715-11R are represented by shallow-water limestone barren of calcareous nannofossils. 


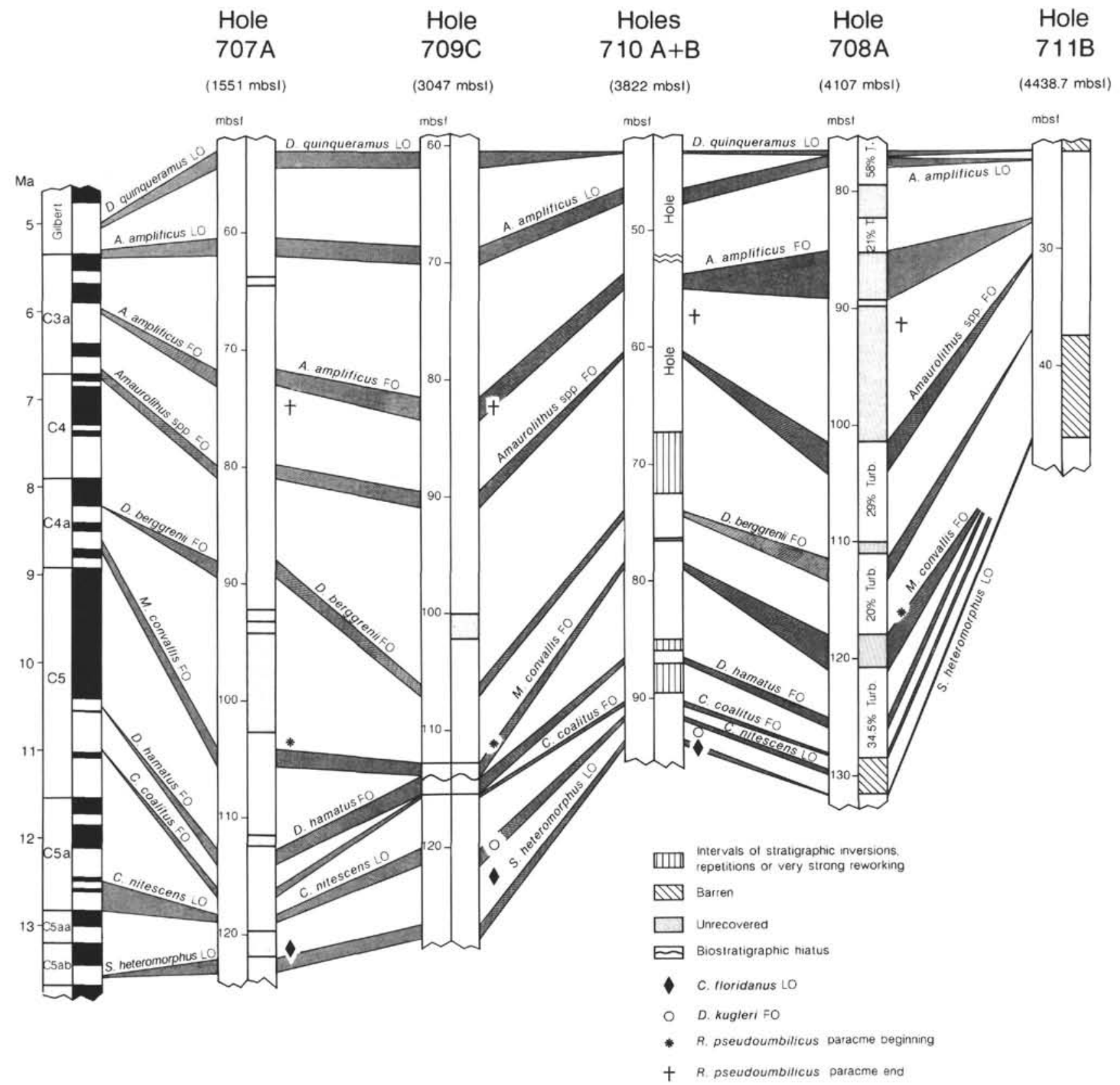

Figure 16. Middle-late Miocene correlations among CBT sites. Major stratigraphic features and main biostratigraphic events are shown.

\section{Comparison between Sequences of Sites 714 and 715}

The two sequences retrieved at nearby Sites 714 and 715 are similar but not identical. Whereas sediments below the megahiatus at Site 714 belong to Zone NN10 (early late Miocene), sediments underlying the megahiatus at Site 715 belong to Zone NN5 (early middle Miocene) (Figs. 20 and 21). The early Miocene sections at the two sites are compared in Figure 22. The intervening events between the S. heteromorphus FO and the $H$. ampliaperta $\mathrm{LO}$ fit a single regression line rather well, indicating that sedimentation was continuous in this interval, although somewhat higher at Site 714. In the underlying interval, the LO of $T$. carinatus occurs below the LO of S. belemnos at Site 715, whereas the two events are virtually simultaneous at Site 714 . This would indicate a minor hiatus or loss of sediments at core boundary in correspondence with the short Zone NN3 at Site 714. The sedimentation rates change definitively between the $S$. belemnos FO and the $S$. heteromorphus FO, becoming higher at Site 715 .

\section{Biochronologic Considerations}

The poor magnetic properties of the retrieved sediments at Sites 714 and 715 did not allow us to derive a reliable magnetic stratigraphy (Schneider and Kent, this volume). This is particularly unfortunate because a complete lower and middle Miocene series is obtained by combining the sequences retrieved at the two sites, in which all the primary and secondary nannofossil events in this critical interval are observed: the LOs of $C$. nitescens, $H$. ampliaperta, S. belemnos, and T. carinatus and the FO of $S$. belemnos.

The ages of these events were estimated by interpolating the sedimentation rate between events calibrated in the equatorial Indian Ocean at Site 710. In Figure 23 we have plotted sedimentation rate at Site 714 , using the $D$. hamatus FO and the $\mathrm{LO}$ and 


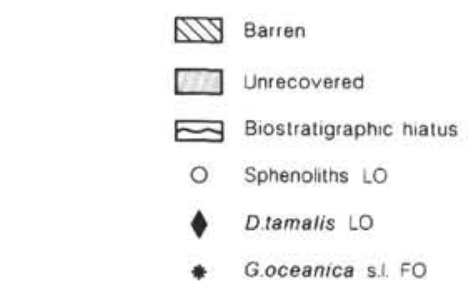

mbst

\section{Holes \\ $709 \mathrm{~A}+\mathrm{C}$}

(3047 mbsl)

mbs!

Hole

707A

(1551 mbsl)

$\mathrm{Ma}$

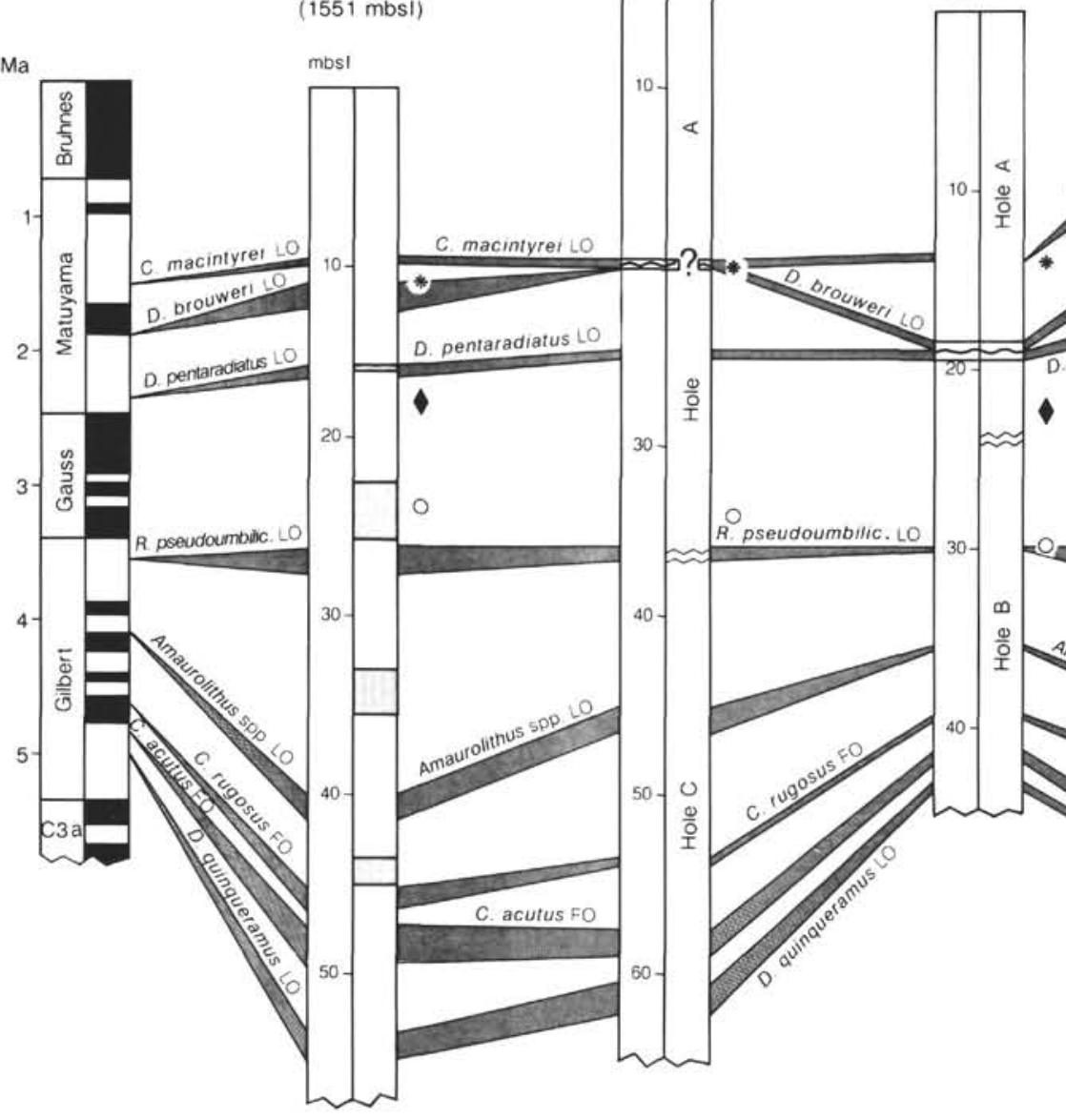

Holes

$710 \mathrm{~A}+\mathrm{B}$

(3822 mbsi)

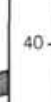

$40-$

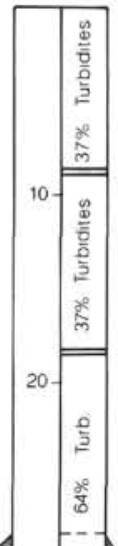

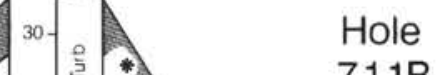

711B

(4438.7 mbsf)

mbs!

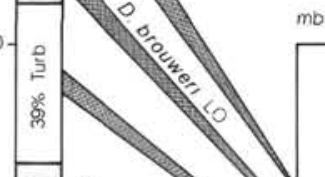

10-

Figure 17. Pliocene correlations among CBT sites. Major stratigraphic features and main biostratigraphic events are shown.

FO of $S$. heteromorphus as control points. The sedimentation rate appears constant. The LO of $C$. nitescens has an interpolated age of $12.68 \pm 0.17 \mathrm{Ma}$. By interpolating the sedimentation rate between the FO of $S$. heteromorphus and the FO of $C$. coalitus at Hole 714A (which has a better recovery), an age of $12.60 \pm 0.15 \mathrm{Ma}$ was obtained (Fig. 23). Previously, Gartner and Chow (1985) suggested an age of about $13 \mathrm{Ma}$ for this event.

The interpolated age of the LO of $H$. ampliaperta is $15.75 \pm$ $0.1 \mathrm{Ma}$. Previously, Takayama and Sato (1985) suggested an age of about $16.0 \mathrm{Ma}$ for this event in the northern Atlantic Ocean.
The acme end of $D$. deflandrei and the coincident FO of $D$. signus $-D$. tuberi have an interpolated age of $16.05 \pm 0.5 \mathrm{Ma}$ (Fig. 23 and Table 2). At Hole 710A, these events occur in an interval of no magnetic stratigraphy, above the negative interval at the base of Chron C5B (Fig. 8), which is in good agreement with the age interpolated at Site 714 .

To obtain estimates of the controversial ages of the early Miocene calcareous nannofossil events (see Table 2), the sedimentation rate between the LO of $H$. ampliaperta and the FO of $S$. heteromorphus was plotted at Site 715 (Fig. 24; Table 2). Unfortunately, the available samples are widely spaced, and the ages 


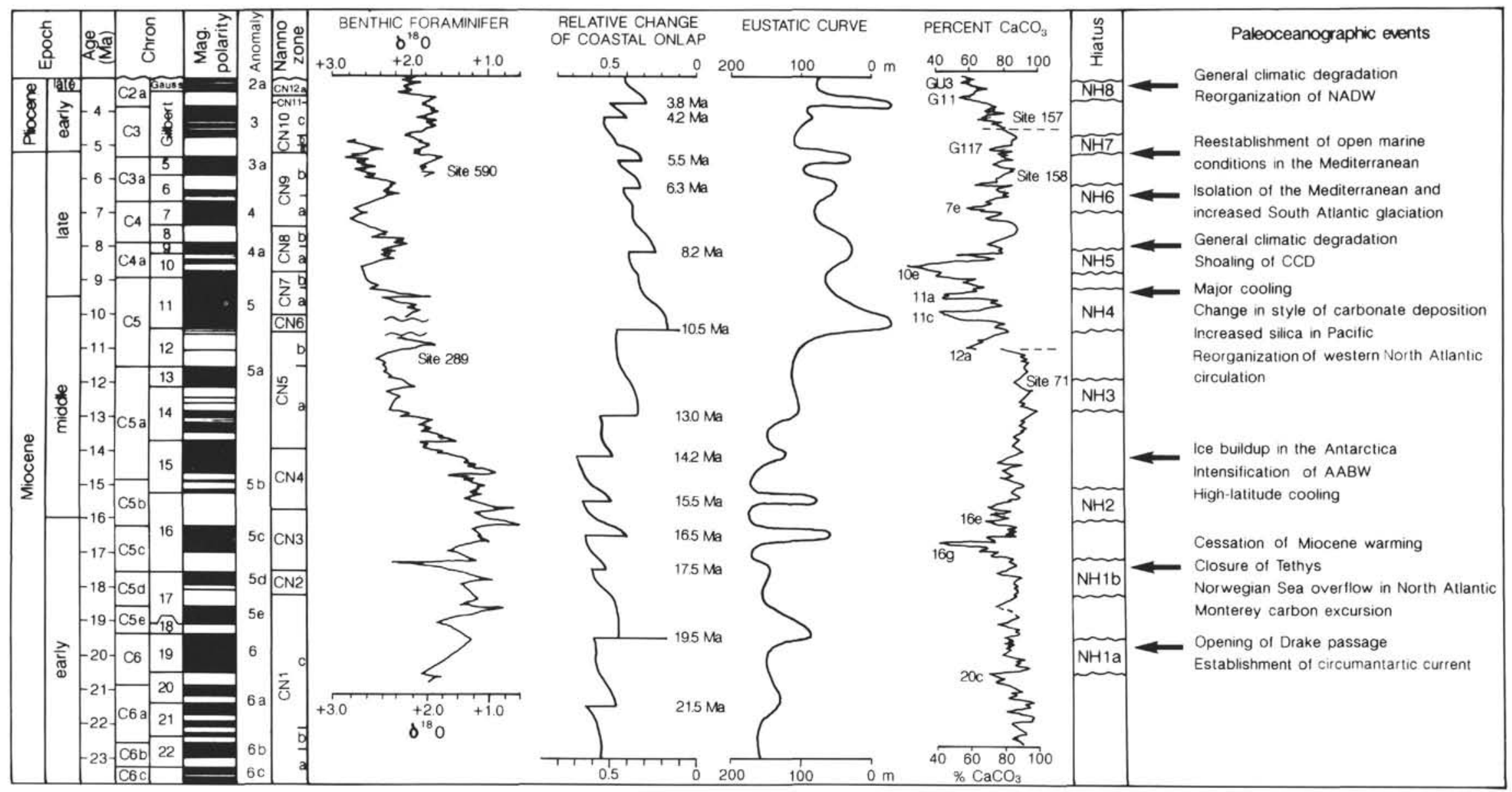

Figure 18. Summary of the Miocene-early Pliocene environmental evolution compiled after Keller and Barron (1987) and Mayer et al. (1986). Note that the magnetobiochronology in this figure is after Keller and Barron (1987) and is based on (1) Tauxe et al. (1983), (2) La Brecque et al. (1977), and on (3) Berggren et al. (1985). Calcareous nannofossil zones are those of Bukry (1973) as coded by Okada and Bukry (1980). The benthic foraminifers oxygen isotope curve is after Woodruff et al. (1981). The coastal onlap and the eustatic curves are after Haq et al. (1987). The percent carbonate curve, in the equatorial Pacific, is after Dunn (1982) and Bode and Cronin (1973). 
Table 23. Summary of the position of calcareous nannofossil events at Hole 712A.

\begin{tabular}{|c|c|c|}
\hline Event & $\begin{array}{l}\text { Core, section, } \\
\text { interval }(\mathrm{cm})\end{array}$ & $\begin{array}{l}\text { Depth } \\
\text { (mbsf) }\end{array}$ \\
\hline Sphenoliths LO & $115-712$ A-1R-3, 50/115-712A-1R-2, 70 & $3.5-2.2$ \\
\hline R. pseudoumbilicus LO & $115-712 \mathrm{~A}-1 \mathrm{R}-4,70 / 115-712 \mathrm{~A}-1 \mathrm{R}-3,50$ & $5.2-3.5$ \\
\hline Amaurolithus spp. LO & $115-712 \mathrm{~A}-2 \mathrm{R}-1,75 / 115-712 \mathrm{~A}-1 \mathrm{R}-7,20$ & $10.15-9.2$ \\
\hline C. acutus LO & $115-712 \mathrm{~A}-2 \mathrm{R}-1,75 / 115-712 \mathrm{~A}-1 \mathrm{R}-7,20$ & $10.15-9.2$ \\
\hline C. rugosus $\mathrm{FO}$ & $115-712 \mathrm{~A}-2 \mathrm{R}-\mathrm{CC} / 115-712 \mathrm{~A}-2 \mathrm{R}-5,75$ & $19.0-16.15$ \\
\hline C. acutus FO & ND & \\
\hline T. rugosus LO & $115-712 \mathrm{~A}-2 \mathrm{R}-\mathrm{CC} / 115-712 \mathrm{~A}-2 \mathrm{R}-5,75$ & $19.0-16.15$ \\
\hline D. quinqueramus LO & $115-712 \mathrm{~A}-3 \mathrm{R}-3,75 / 115-712 \mathrm{~A}-3 \mathrm{R}-2,75$ & $22.75-21.25$ \\
\hline A. amplificus & Recorded only in Sample 115-712A-4R-2, 75 (30.85 mbsf) & \\
\hline Amaurolithus spp. FO & $115-712$ A- 4 R- $2,93 / 115-712$ A- 4 R- 2,80 & $31.03-30.90$ \\
\hline D. berggrenii $\mathrm{FO}$ & $115-712$ A-4R-2, $93 / 115-712$ A-4R-2, 80 & $31.03-30.90$ \\
\hline M. convallis LO & $115-712 \mathrm{~A}-4 \mathrm{R}-2,102 / 115-712 \mathrm{~A}-4 \mathrm{R}-2,93$ & $31.12-31.03$ \\
\hline M. convallis FO & 115-712A-5R-1, 130/115-712A-5R-1, 75 & $39.6-39.05$ \\
\hline D. hamatus LO & $115-712 \mathrm{~A}-5 \mathrm{R}-1,130 / 115-712 \mathrm{~A}-5 \mathrm{R}-1,75$ & $39.6-39.05$ \\
\hline C. calyculus & Present in Sample 115-712A-5R-1, 30 (39.6 mbsf) & \\
\hline D. hamatus FO & $115-712 \mathrm{~A}-6 \mathrm{R}-1,75 / 115-712 \mathrm{~A}-5 \mathrm{R}-2,75$ & $48.65-40.55$ \\
\hline S. heteromorphus & $115-712 \mathrm{~A}-6 \mathrm{R}-1,75 / 115-712 \mathrm{~A}-5 \mathrm{R}-\mathrm{CC}$ & $48.65-47.90$ \\
\hline
\end{tabular}

Note: $\mathrm{LO}=$ last occurrence, $\mathrm{FO}=$ first occurrence, and $\mathrm{ND}=$ not detected.

Table 24. Summary of the position of calcareous nannofossil events at Hole 713A.

\begin{tabular}{lcc}
\hline \multicolumn{1}{c}{ Event } & \multicolumn{1}{c}{$\begin{array}{c}\text { Core, section, } \\
\text { interval }(\mathrm{cm})\end{array}$} & $\begin{array}{c}\text { Depth } \\
\text { (mbsf) }\end{array}$ \\
\hline C. macintyrei LO & $115-713 \mathrm{~A}-2 \mathrm{R}-2,130 / 115-713 \mathrm{~A}-2 \mathrm{R}-2,50$ & $4.4-3.6$ \\
G. oceanica FO & $115-713 \mathrm{~A}-2 \mathrm{R}-2,130 / 115-713 \mathrm{~A}-2 \mathrm{R}-2,50$ & $4.4-3.6$ \\
D. brouweri LO & $115-713 \mathrm{~A}-2 \mathrm{R}-2,130 / 115-713 \mathrm{~A}-2 \mathrm{R}-2,50$ & $4.4-3.6$ \\
D. triradiatus LO & $115-713 \mathrm{~A}-2 \mathrm{R}-2,130 / 115-713 \mathrm{~A}-2 \mathrm{R}-2,50$ & $4.4-3.6$ \\
D. pentaradiatus LO & $115-713 \mathrm{~A}-2 \mathrm{R}-3,130 / 115-713 \mathrm{~A}-2 \mathrm{R}-3,50$ & $5.9-5.1$ \\
D. surculus LO & $115-713 \mathrm{~A}-2 \mathrm{R}-4,50 / 115-713 \mathrm{~A}-2 \mathrm{R}-3,130$ & $6.6-5.9$ \\
D. tamalis LO & $115-713 \mathrm{~A}-2 \mathrm{R}-4,50 / 115-713 \mathrm{~A}-2 \mathrm{R}-3,130$ & $6.6-5.9$ \\
Sphenoliths LO & $115-713 \mathrm{~A}-3 \mathrm{R}-1,50 / 115-713 \mathrm{~A}-2 \mathrm{R}-6,50$ & $11.7-9.6$ \\
R. pseudoumbilicus LO & $115-713 \mathrm{~A}-3 \mathrm{R}-1,50 / 115-713 \mathrm{~A}-2 \mathrm{R}-6,50$ & $11.7-9.6$ \\
C. rugosus FO & $115-713 \mathrm{~A}-3 \mathrm{R}-6,75 / 115-713 \mathrm{~A}-3 \mathrm{R}-5,130$ & $19.45-18.5$ \\
C. acutus FO & $115-713 \mathrm{~A}-3 \mathrm{R}-6,130 / 115-713 \mathrm{~A}-3 \mathrm{R}-6,75$ & $20.0-19.45$ \\
T. rugosus LO & $115-713 \mathrm{~A}-3 \mathrm{R}-6,130 / 115-713 \mathrm{~A}-3 \mathrm{R}-6,75$ & $20.0-19.45$ \\
D. quinqueramus LO & $115-713 \mathrm{~A}-3 \mathrm{R}-6,130 / 115-713 \mathrm{~A}-3 \mathrm{R}-6,75$ & $20.0-19.45$ \\
A. amplificus LO & $115-713 \mathrm{~A}-3 \mathrm{R}-6,130 / 115-713 \mathrm{~A}-3 \mathrm{R}-6,75$ & $20.0-19.45$ \\
A. amplificus FO & $115-713 \mathrm{~A}-4 \mathrm{R}-5,50 / 115-713 \mathrm{~A}-4 \mathrm{R}-4,130$ & $27.30-26.60$ \\
Amaurolithus spp. FO & $115-713 \mathrm{~A}-4 \mathrm{R}-7,20$ & 30.0 \\
D. berggrenii FO & $115-713 \mathrm{~A}-4 \mathrm{R}-7,20$ & 30.0 \\
\hline
\end{tabular}

Note: $L O=$ last occurrence and $F O=$ first occurrence.

obtained are not well constrained. Yet the age estimates obtained indicate that the interval between the FO and the LO of $S$. belemnos (Zone $\mathrm{CN} 2$ ) is very short, in agreement with Barron et al. (1985) and contrary to what was suggested by Berggren et al. (1985) (see Table 2). The age obtained for the FO of $S$. belemnos is $19.35 \pm 0.2 \mathrm{Ma}$ (i.e., close to the transition between Chrons C6 and C5E; Fig. 2). This interpolated age is in agreement with the fact that at Site 710 we did not detect $S$. belemnos within Chron C6 (Fig. 8).

The LO of $T$. carinatus seems to occur at $18.95 \pm 0.25 \mathrm{Ma}$. The form is indeed abundant during the Chron C6 time interval at Site 710 (Fig. 8). If the ages suggested here for the FO of $S$. belemnos and the LO of T. carinatus are correct, we are faced with a very low calcareous nannofossil biostratigraphic resolution in the early Miocene (Fig. 1). In addition, the events used in the standard zonations, the FO of $D$. druggii and the acme end of $C$. abisectus, are difficult to recognize in many areas and are poorly constrained in age.

\section{Site 716}

Site 716 is located in flat terrain on a broad, shallow basin in the center of the Maldives Ridge (Fig. 1 and Table 1). Two holes were continuously APC cored, Hole $716 \mathrm{~A}$ to $257 \mathrm{mbsf}$ and Hole $716 \mathrm{~B}$ to $267.4 \mathrm{mbsf}$. Recovery was in excess of $100 \%$. The lithology is represented by foraminifer-bearing nannofossil ooze that grades into chalk downhole.

For the present work, we studied 80 samples from the late Miocene-Pliocene sequence retrieved at Hole 716B. Calcareous nannofossils are abundant throughout the sequence. The preservation state is very poor because of overgrowth, which prevents identification of many marker species (i.e., ceratoliths and discoasterids). The events that have been recognized confidently are summarized in Table 30.

\section{SUMMARY AND CONCLUSIONS}

We have presented the calcareous nannofossil biostratigraphic and the chronostratigraphic classification of the latest Oligocene to the earliest Pleistocene sediments retrieved during Leg 115 in the western equatorial Indian Ocean. Most of the zonal boundaries of the standard zonations of Martini (1971) and of Okada and Bukry (1980), are easily recognized in these low-latitude oceanic sediments.

Pliocene Zones NN13 and NN14 and Subzones CN11a and CN11b could not be differentiated because of the sporadic occurrence of $D$. asymmetricus. Middle-late Miocene Subzones $\mathrm{CN} 8 \mathrm{a} / \mathrm{CN} 8 \mathrm{~b}$ and $\mathrm{CN} 7 \mathrm{a} / \mathrm{CN} 7 \mathrm{~b}$ were not recognized because of the sporadic occurrence or different stratigraphic ranges of the primary and secondary species $(D$. loeblichii, $D$. neorectus, and C. calyculus) used for their definition.

The boundary between Zones NN4 and NN5 and the boundary between Zones $\mathrm{CN} 3$ and $\mathrm{CN} 4$, defined by the $\mathrm{LO}$ of $\mathrm{H}$. ampliaperta, were recognized by means of their primary definition only at Maldives Sites 714 and 715. At these sites the LO of $H$. ampliaperta occurs slightly above the acme end of $D$. deflandrei and the FO of $D$. signus-D. tuberi, which were adopted for the recognition of the $\mathrm{NN} 4 / \mathrm{NN} 5$ and $\mathrm{CN} 3 / \mathrm{CN} 4$ boundaries at other sites. Subzones CN1a and CN1b were not differentiated because $C$. abisectus becomes extinct in the area before or together with $S$. ciperoensis. The boundary between Zones NP25 and NN1 was recognized by means of the LO of S. ciperoensis because $H$. recta is missing or very rare.

We have discussed the secondary criteria proposed by Bukry $(1973,1975,1978,1985)$ for the recognition of his zonal boundaries, and we have discussed supplementary events that are potentially useful for improving the biostratigraphic resolution provided by the standard zonations.

In the late Miocene Subzone CN9b, the FO and LO of $A$. amplificus are consistently applied for intersite correlations in 


\section{Hole 712A}

Hole 713A

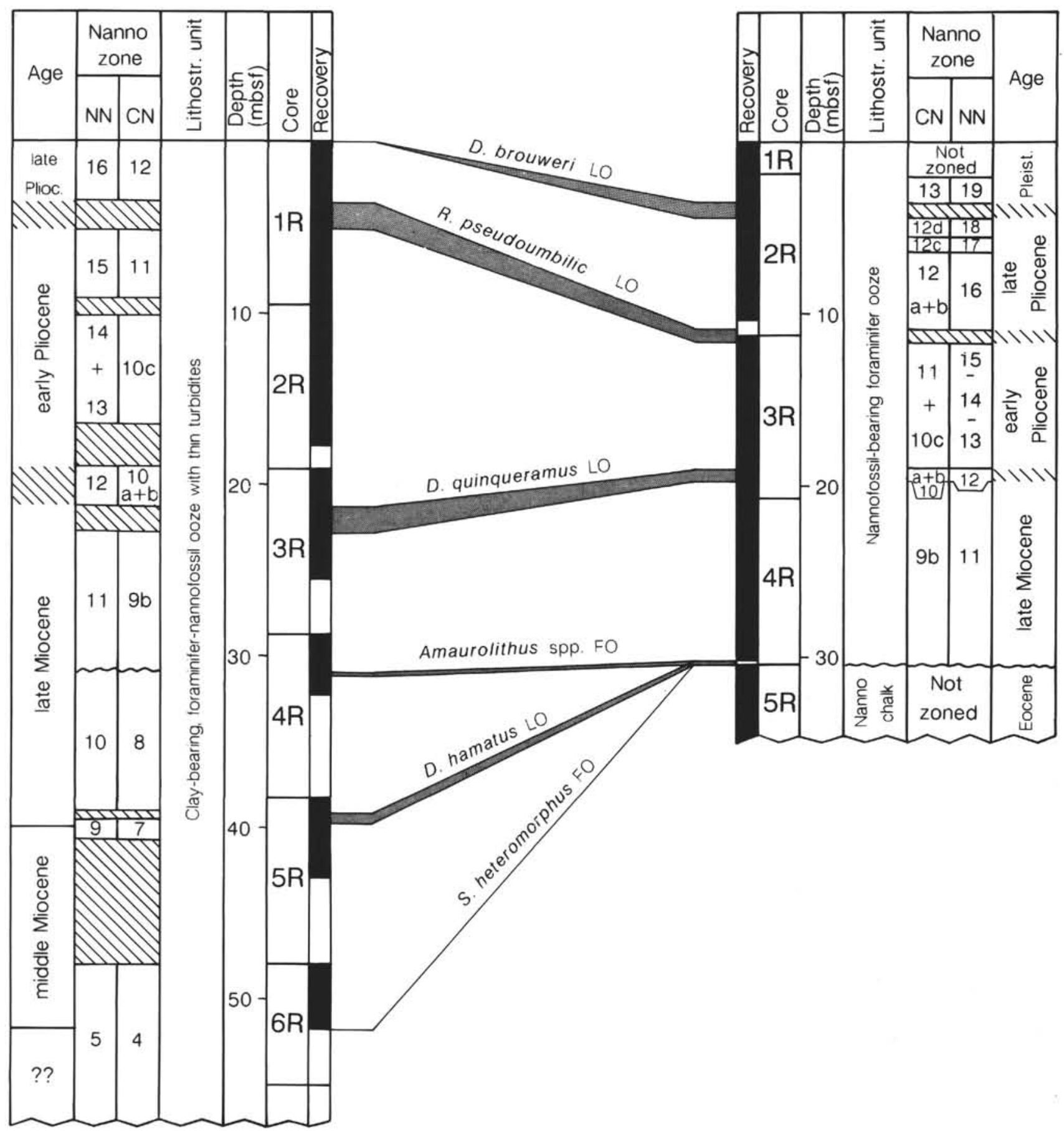

Figure 19. Correlation between Chagos Sites 712 and 713 on the basis of calcareous nannofossils.

the area, and they may also be useful elsewhere. The FO of $M$. convallis seems to be a good event to approximate the NN9/ $\mathrm{NN} 10$ and $\mathrm{CN} 7 / \mathrm{CN} 8$ boundaries. The LO of $C$. nitescens is easily determined and correlated at all sites, occurring close to the FO of $D$. kugleri. Since the latter event is difficult to recognize in many areas, the NN6/NN7 and CN5a/CN5b boundaries may be approximated by the $\mathrm{LO}$ of $C$. nitescens.

The LO of $C$. floridanus, proposed as a secondary event to recognize the $\mathrm{CN} 5 \mathrm{a} / \mathrm{CN} 5 \mathrm{~b}$ boundary, is easily detected, but it is recorded slightly above the LO of $S$. heteromorphus and well below the FO of $D$. kugleri. The data available indicate that this event is probably diachronous in the different areas. A correlatable short-lived acme interval of $S$. delphix was recorded at all sites in the upper part of Zone NN1 (CN1c). The form is restricted to this zonal interval in the area.

The study of the calcareous nannofossil biostratigraphy and the taphonomy of the sequences from the CBT sites (Northern Mascarene Plateau area), which were drilled to investigate the 


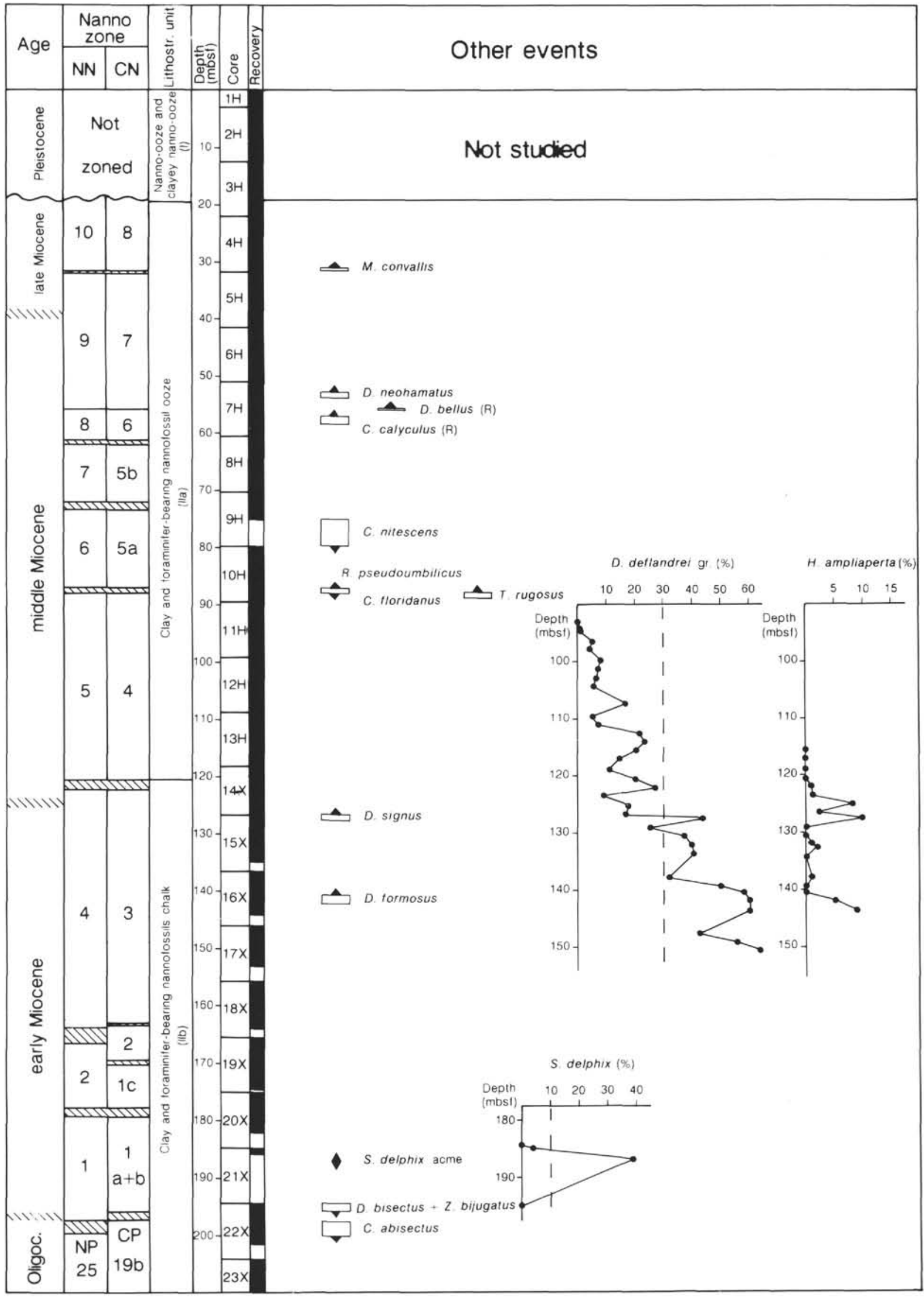

Figure 20. Chronostratigraphy and calcareous nannofossil biostratigraphy at Hole 714A. For legend, see Figure 3. 
Table 26. Summary of the position of calcareous nannofossil events at Hole 714A.

\begin{tabular}{|c|c|c|}
\hline Event & $\begin{array}{l}\text { Core, section, } \\
\text { interval }(\mathrm{cm})\end{array}$ & $\begin{array}{l}\text { Depth } \\
\text { (mbsf) }\end{array}$ \\
\hline convallis FO & $115-714 \mathrm{~A}-4 \mathrm{H}-7,140 / 115-714 \mathrm{~A}-4 \mathrm{H}-7,75$ & $31.7-31.05$ \\
\hline D. hamatus LO & $115-714 \mathrm{~A}-5 \mathrm{H}-1,30 / 115-714 \mathrm{~A}-4 \mathrm{H}-7,140$ & $32.0-31.7$ \\
\hline D. neohamatus FO & $115-714 \mathrm{~A}-7 \mathrm{H}-2,140 / 115-714 \mathrm{~A}-7 \mathrm{H}-2,30$ & $53.9-52.8$ \\
\hline D. hamatus FO & $115-714 \mathrm{~A}-7 \mathrm{H}-4,30 / 115-714 \mathrm{~A}-7 \mathrm{H}-4,10$ & $55.8-55.6$ \\
\hline C. calyculus FRO & $115-714 \mathrm{~A}-7 \mathrm{H}-5,140 / 115-714 \mathrm{~A}-7 \mathrm{H}-4,140$ & $58.4-56.9$ \\
\hline C. coalitus FO & $115-714 \mathrm{~A}-8 \mathrm{H}-1,140 / 115-714 \mathrm{~A}-8 \mathrm{H}-1,75$ & $62.0-61.35$ \\
\hline D. kugleri FO & $115-714 \mathrm{~A}-9 \mathrm{H}-3,10 / 115-714 \mathrm{~A}-9 \mathrm{H}-2,30$ & $73.30-72.0$ \\
\hline C. nitescens LO & $115-714 \mathrm{~A}-9 \mathrm{H}-\mathrm{CC} / 115-714 \mathrm{~A}-9 \mathrm{H}-5,30$ & $79.8-76.5$ \\
\hline C. floridanus LO & $115-714 \mathrm{~A}-10 \mathrm{H}-6,75 / 115-714 \mathrm{~A}-10 \mathrm{H}-5,130$ & $88.05-87.1$ \\
\hline S. heteromorphus LO & $115-714 \mathrm{~A}-10 \mathrm{H}-6,75 / 115-714 \mathrm{~A}-10 \mathrm{H}-5,130$ & $88.05-87.1$ \\
\hline R. pseudoumbilicus FO & $115-714 \mathrm{~A}-10 \mathrm{H}-6,75 / 115-714 \mathrm{~A}-10 \mathrm{H}-5,130$ & $88.05-87.1$ \\
\hline H. ampliaperta LO & $115-714 \mathrm{~A}-14 \mathrm{X}-3,75 / 115-714 \mathrm{~A}-14 \mathrm{X}-2,75$ & $122.15-120.65$ \\
\hline D. signus FO & $115-714 \mathrm{~A}-15 \mathrm{X}-1,75 / 115-714 \mathrm{~A}-14 \mathrm{X}-6,75$ & $127.55-126.75$ \\
\hline D. deflandrei acme end & $115-714 \mathrm{~A}-15 \mathrm{X}-1,75 / 115-714 \mathrm{~A}-14 \mathrm{X}-6,75$ & $127.55-126.75$ \\
\hline D. formosus FO & $115-714 \mathrm{~A}-16 \mathrm{X}-4,130 / 115-714 \mathrm{~A}-16 \mathrm{X}-3,130$ & $142.2-140.7$ \\
\hline S. heteromorphus FO & $115-714 \mathrm{~A}-18 \mathrm{X}-6,10 / 115-714 \mathrm{~A}-18 \mathrm{X}-5,130$ & $163.3-163.0$ \\
\hline S. belemnos LO & $115-714 \mathrm{~A}-19 \mathrm{X}-1,130 / 115-714 \mathrm{~A}-18 \mathrm{X}-\mathrm{CC}$ & $166.6-165.3$ \\
\hline T. carinatus LO & $115-714 \mathrm{~A}-19 \mathrm{X}-1,130 / 115-714 \mathrm{~A}-18 \mathrm{X}-\mathrm{CC}$ & $166.6-165.3$ \\
\hline S. belemnos $\mathrm{FO}$ & $115-714 \mathrm{~A}-19 \mathrm{X}-4,130 / 115-714 \mathrm{~A}-19 \mathrm{X}-3,130$ & $171.1-169.6$ \\
\hline D. druggii $\mathrm{FO}$ & $115-714 \mathrm{~A}-20 \mathrm{X}-3,130 / 115-714 \mathrm{~A}-20 \mathrm{X}-2,130$ & $179.3-177.8$ \\
\hline C. abisectus LO & $115-714 \mathrm{~A}-22 \mathrm{X}-4,120 / 115-714 \mathrm{~A}-22 \mathrm{X}-3,10$ & $200.1-197.5$ \\
\hline S. ciperoensis LO & $115-714 \mathrm{~A}-22 \mathrm{X}-2,130 / 115-714 \mathrm{~A}-22 \mathrm{X}-1,130$ & $197.2-195.7$ \\
\hline D. bisectus LO & $115-714 \mathrm{~A}-22 \mathrm{X}-1,130 / 115-714 \mathrm{~A}-21 \mathrm{X}-\mathrm{CC}$ & $195.7-194.4$ \\
\hline Z. bijugatus LO & $115-714 \mathrm{~A}-22 \mathrm{X}-1,130 / 115-714 \mathrm{~A}-21 \mathrm{X}-\mathrm{CC}$ & $195.7-194.4$ \\
\hline H. recta LO & $115-714 \mathrm{~A}-22 \mathrm{X}-4,120 / 115-714 \mathrm{~A}-22 \mathrm{X}-3,10$ & 200.1-197.5 \\
\hline
\end{tabular}

Note: $\mathrm{LO}=$ last occurrence and $\mathrm{FO}=$ first occurrence.

Table 27. Summary of the position of calcareous nannofossil events at Hole 714B.

\begin{tabular}{llc}
\hline \multicolumn{1}{c}{ Event } & \multicolumn{1}{c}{$\begin{array}{c}\text { Core, section, } \\
\text { interval }(\mathrm{cm})\end{array}$} & $\begin{array}{c}\text { Depth } \\
\text { (mbsf) }\end{array}$ \\
\hline D. hamatus LO & $115-714 \mathrm{~B}-4 \mathrm{H}-4,40 / 115-714 \mathrm{~B}-3 \mathrm{H}-\mathrm{CC}$ & $30.9-26.0$ \\
D. hamatus FO & $115-714 \mathrm{~B}-6 \mathrm{H}-7,40 / 115-714 \mathrm{~B}-6 \mathrm{H}-6,40$ & $54.8-53.3$ \\
C. coalitus FO & $115-714 \mathrm{~B}-7 \mathrm{H}-5,40 / 115-714 \mathrm{~B}-7 \mathrm{H}-4,40$ & $61.4-59.9$ \\
D. kugleri FO & $115-714 \mathrm{~B}-8 \mathrm{H}-6,40 / 115-714 \mathrm{~B}-8 \mathrm{H}-5,40$ & $72.5-71.0$ \\
C. nitescens LO & $115-714 \mathrm{~B}-9 \mathrm{H}-3,40 / 115-714 \mathrm{~B}-9 \mathrm{H}-2,40$ & $77.7-76.2$ \\
C. floridanus LO & $115-714 \mathrm{~B}-10 \mathrm{H}-3,40 / 115-714 \mathrm{~B}-10 \mathrm{H}-2,40$ & $87.3-85.8$ \\
S. heteromorphus LO & $115-714 \mathrm{~B}-10 \mathrm{H}-4,40 / 115-714 \mathrm{~B}-10 \mathrm{H}-3,40$ & $88.8-87.3$ \\
R. pseudoumbilica FO & $115-714 \mathrm{~B}-10 \mathrm{H}-4,40 / 115-714 \mathrm{~B}-10 \mathrm{H}-3,40$ & $88.8-87.3$ \\
\hline
\end{tabular}

Note: $L O=$ last occurrence and FO = first occurrence.

Neogene history of carbonate flux and dissolution, indicates that the accumulation of carbonates in this area results from a complex interplay among carbonate bioproductivity, carbonate removal by chemical dissolution and mechanical erosion, and carbonate addition by mass and current transport. Biostratigraphic discontinuities, biostratigraphic inversions, and strong reworking are present at the CBT sites, which prohibit us from considering carbonate accumulation as a linear response to carbonate production and dissolution. In spite of the many drawbacks, major changes and trends in the Neogene carbonate accumulation history can be recognized in the sedimentary record of the CBT sites, most of which correlate to major steps in the evolution of the Neogene climatic system.

In the early Miocene, at about $20 \mathrm{Ma}$, a drop in carbonate accumulation and a shallowing of the $C C D$ was recorded that is roughly correlatable to a major plate reorganization. In the early middle Miocene (upper part of Zones CN3 and CN4), an amelioration in carbonate accumulation is recorded that correlates to a high sea-level stand, which could be related to a fractionation of organic carbon in the shelf and reduced dissolution in the deep sea (Broecker, 1982).

In the middle Miocene (Zones CN5 through CN8), a severe deep-sea carbonate crisis was recorded, with sedimentation of residual facies virtually at all intermediate and deep sites. A shallow CCD during most of this time interval is a global phenomenon. The beginning of this interval correlates precisely with a permanent increase in benthic foraminifers $\delta^{18} \mathrm{O}$, generally interpreted as caused by enhanced ice growth in the Antarctic.

A carbonate optimum is recorded in the late Miocene (Zone CN9), when an increase in carbonate accumulation is recorded at all sites. The beginning of this interval correlates to a further change in the benthic foraminifers $\delta^{18} \mathrm{O}$ record.

After an early Pliocene shallowing, the CCD dropped to the deep present-day values at about $3.0 \mathrm{Ma}$, that is, in coincidence with another change in the climatic system. This change slightly precedes the definitive onset of Northern Hemisphere glaciation at about $2.5 \mathrm{Ma}$.

\section{TAXONOMIC NOTES}

Remarks on taxonomic concepts followed and used in preparing range charts are given below together with information on stratigraphic distributions of the most important taxa. A complete list of taxa considered in this report can be found in the Appendix.

\section{Discoasterids}

Discoasterids are well represented in the sediments retrieved during Leg 115 except at Site 716 (located in a periplatform setting), where they represent only a small percentage of the assemblage. It is well known that discoasterids are dissolution resistant and prone to secondary overgrowth (Wise, 1973; Bukry, 1978). In overgrown assemblages, the identification of discoasterids at the specific level is difficult and sometimes impossible. Consequently, it is impossible to quantify the real abundance of the different species. Since the abundance data reported in the range charts refer only to specimens identified confidently, they are underestimated, particularly in overgrown material. In many instances, because of species circumscription problems, we have used groupings of undetermined discoasterids in the range charts (i.e., "six-rayed undetermined discoasterids").

\section{Pliocene Discoasterids}

Pliocene discoasterid species have been identified following concepts commented on in Backman and Shackleton (1983). It should be noted that the assemblages are dominated by Discoaster brouweri and $D$. pentaradiatus. Discoaster surculus, $D$. variabilis, D. decorus, and, overall, D. tamalis and D. asymmetricus are subordinate or rare. Rio et al. (in press c) indicate for the latter two species a preference for cooler water masses.

\section{Middle-Late Miocene Discoasterids}

A major turnover in the discoasterid assemblages occurred in the early middle Miocene, when massive forms were gradually replaced by more slender forms that characterize the final range of the group (Bukry, 1971). We comment on the middle-late Miocene discoasterids by subdividing them into the following morphologic groups.

\section{Five-pointed Ray Discoasterids}

This group includes: D. hamatus, D. bellus, D. berggrenii, D. quinqueramus, and Discoaster sp. 1.

Discoaster hamatus (Plate 1, Figs. 1-3) is easily distinguished by the long rays that turn sharply clockwise near the tips. At the tip a small spine is present that extends in the direction of the ray. We have restricted our species concept to forms with five arms (following Perch-Nielsen, 1985), even if D. hamatus was originally described as a five- and six-rayed form. Indeed, rare six-rayed discoasterids, with a structure comparable in size and proportion to the five-rayed D. hamatus were recorded (Plate 1, Fig. 5); these morphotypes have been found together with $D$. neohamatus and $D$. calcaris, and discrimination among these three species is often difficult and subjective. 


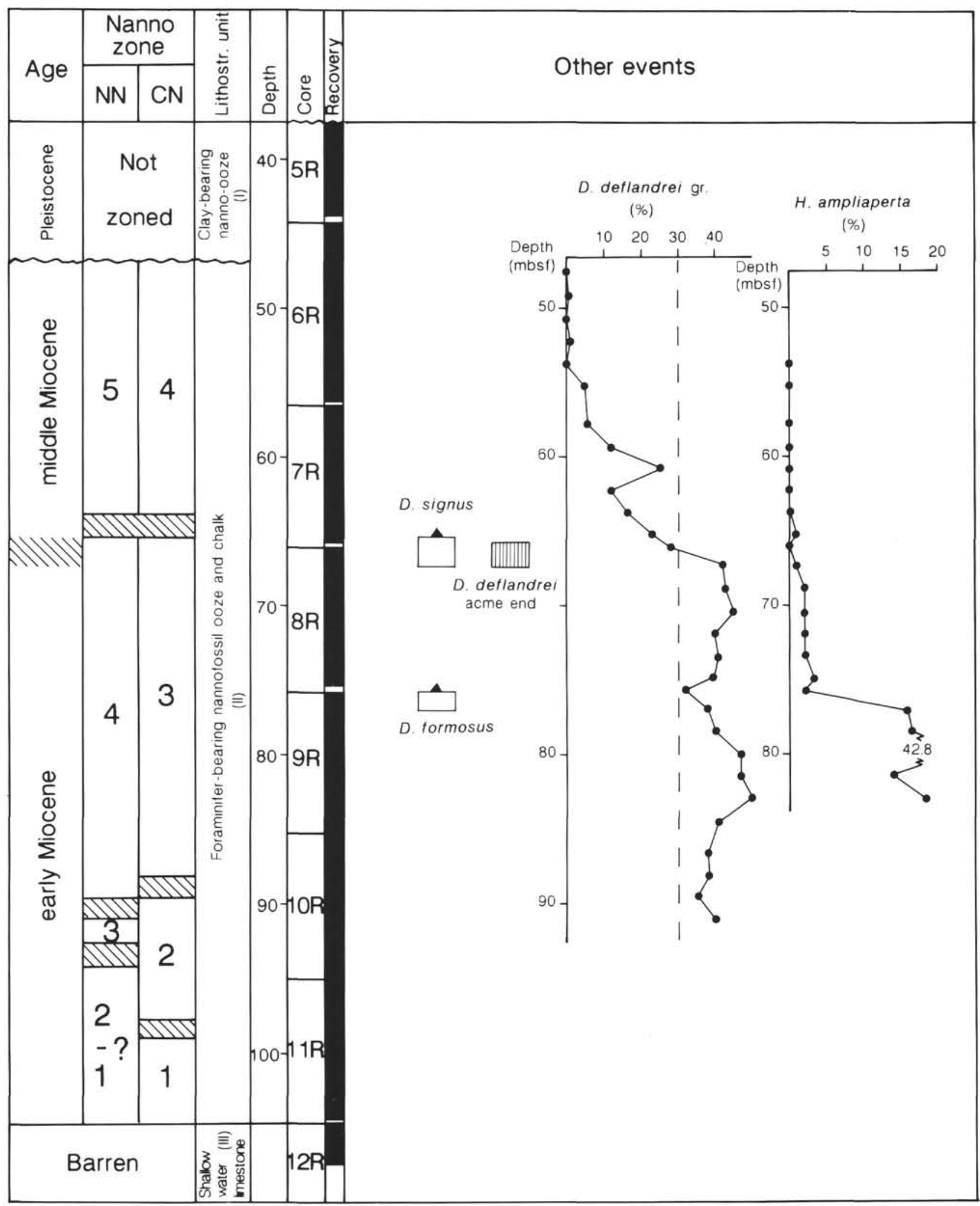

Figure 21. Chronostratigraphy and calcareous nannofossil biostratigraphy at Site 715. For legend, see Figure 3. 
Table 29. Summary of the position of calcareous nannofossil events at Hole 715A.

\begin{tabular}{llc}
\hline \multicolumn{1}{c}{ Event } & \multicolumn{1}{c}{$\begin{array}{c}\text { Core, section, } \\
\text { interval }(\mathrm{cm})\end{array}$} & $\begin{array}{c}\text { Depth } \\
\text { (mbsf) }\end{array}$ \\
\hline S. heteromorphus LO & \multicolumn{1}{c}{ 115-715A-5R-6, 130/115-715A-6R-1, 90 } & $45.9-47.7$ \\
H. ampliaperta LO & $115-715 \mathrm{~A}-7 \mathrm{R}-6,130 / 115-715 \mathrm{~A}-7 \mathrm{R}-5,130$ & $65.3-63.8$ \\
D. signus FO & $115-715 \mathrm{~A}-8 \mathrm{R}-1,130 / 115-715 \mathrm{~A}-7 \mathrm{R}-6,130$ & $67.4-65.3$ \\
D. deflandrei acme end & 115-715A-8R-1, 130/115-715A-7R-CC & $67.4-66.1$ \\
D. exilis FO & 115-715A-8R-CC/115-715A-8R-5, 130 & $75.7-73.5$ \\
D. formosus FO & 115-715A-9R-1, 130/115-715A-8R-CC & $77.0-75.7$ \\
S. heteromorphus FO & 115-715A-10R-3, 30/115-715A-10R-2, 30 & $89.6-88.1$ \\
S. belemnos LO & 115-715A-10R-4, 130/115-715A-10R-3, 130 & $91.1-89.6$ \\
T. carinatus LO & 115-715A-10R-6, 130/115-715A-10R-5, 130 & $94.1-92.6$ \\
S. belemnos FO & 115-715A-11R-3, 130/115-715A-11R-2, 130 & $99.2-97.7$ \\
D. druggii FO & ND & \\
\hline
\end{tabular}

Note: $L O=$ last occurrence, $F O=$ first occurrence, and ND $=$ not detected.

$\overrightarrow{D i s c o a s t e r}$ bellus is characterized by its small size $(6-8 \mu \mathrm{m})$ and a poorly developed central area (Plate 1, Figs. 6 and 7). It appears together with $D$. hamatus and ranges up to the FO of $D$. berggrenii. Intergrades between $D$. bellus and $D$. hamatus (Plate 1, Fig. 4) and D. bellus and D. berggrenii (Plate 2, Figs. 1 and 2) are present.

We have commented on in the biostratigraphic section that we concur with Bukry (1971) that $D$. berggrenii and $D$. quinqueramus (Plate 2, Figs. 3-9) represent distinct species, because they have a distinct stratigraphic distribution, $D$. berggrenii appearing and becoming extinct earlier than $D$. quinqueramus. Because intergrades between end-members of the $D$. berggreniiD. quinqueramus group are present (Plate 2, Fig. 6), we have lumped the two species together in the range charts. Specimens of $D$. quinqueramus larger than $16 \mu \mathrm{m}$ in size were observed in the final range of the species.

Discoaster sp. 1 in the range charts refers to a five-pointed ray asterolith with a poorly developed central area and a very small central knob (Plate 2, upper right Fig. 9). This discoaster has a smaller size than $D$. berggrenii and $D$. quinqueramus (6-8 $\mu \mathrm{m}$ vs. $8-15 \mu \mathrm{m}$ ) and may represent an intergrade form between $D$. bellus and $D$. quinqueramus. It is abundant and continuously distributed up to the LO of $D$. quinqueramus.

\section{Five-bifurcating Ray Discoasterids}

This group includes Discoaster moorei, D. prepentaradiatus, and $D$. pentaradiatus. Discoaster moorei (Plate 2, Figs. 14 and 15; Plate 11, Fig. 7) is easily recognized by its asymmetrical bifurcating rays, even in overgrown assemblages. It is distributed in low abundances from Zones NN4 to NN7. Discoaster prepentaradiatus (Plate 2, Figs. 12 and 13) has been distinguished from $D$. pentaradiatus on the basis of its poorly developed central area, and its lack of birefringence and downward-bending arms. Discoaster pentaradiatus (Plate 2, Figs. 10 and 11) shows a high morphologic variability; that is rays are more or less slender and bifurcations more or less developed.

\section{Six-pointed Ray Discoasterids}

This morphologic group includes $D$. brouweri, $D$. braarudii, D. intercalaris, D. neohamatus, D. neorectus, and Discoaster sp. 2. The first and continuously distributed typical $D$. brouweri, with downward bending and pointed slender arms, (Plate 3, Figs. 3-5) appears in Zone NN9 (CN7). However, discoasterids of similar morphology that lack the characteristic bending of the rays were observed as low as the upper part of Zone NN5.

Discoaster braarudi, characterized by slender untapering rays and a very small central area, is rare and present consistently only in Zones NN8 and NN9. Intergrade forms to D. brouweri are present, and in some range charts the two forms were lumped together.

The medium-sized $D$. intercalaris is discontinuously occurring and shows a high degree of variability. Typical $D$. neorectus was found to be very rare in spot samples, and it has not been reported in the range charts.

A gigantic discoasterid, morphologically similar to $D$. brouweri, labeled Discoaster sp. 2 in the range charts, was consistently recorded in Subzones CN9a and CN9b (lower part). This form, $20-30 \mu \mathrm{m}$ in size, has six slender rays that are symmetrically arranged and no central area (Plate 3, Figs. 6 and 7). Although found in low abundances, it is easily recognized in the assemblages for its large size.

Discoaster neohamatus was easily recognized, even in overgrown assemblages, because of the long slender rays with pointed tips, sharply bending in one direction, that form a sort of "spine" (Plate 3, Figs. 1 and 2). This characteristic feature is less evident in the specimens found in the terminal range of the species, when intergrade forms to $D$. brouweri are present.

\section{Six-bifurcating Ray Discoasterids}

The species belonging to this morphologic group are D.variabilis, $D$. challengeri, $D$. pansus, $D$. icarus, $D$. perclarus, $D$. loeblichii, D. bollii, D. exilis, D. surculus, D. pseudovariabilis, $D$. subsurculus, D. calcaris, D. aff.calcaris, D. signus, and $D$. tuberi. Most species belonging to this group are not easily differentiated because many intergrading forms exist. We have determined all the above-mentioned species by adhering strictly to the original descriptions, except for $D$. variabilis, which has been intended sensu latu, and $D$. surculus, to which we have assigned discoasterids possessing "trifurcated" tips. The figures in Plates 4, 5, and 6 show our species concepts.

In the range charts, we have tabulated the distribution of forms similar to $D$. calcaris, labeled as $D$. aff. calcaris, which deserve a comment. These forms (Plate 5, Figs. 4-6) are 18-25 $\mu \mathrm{m}$ in size and, therefore, are larger than typical $D$. calcaris $(12-16 \mu \mathrm{m})$. They possess an evident asymmetrical bifurcation as type $D$. calcaris, but the smaller branch does not bend proximally so sharply. Although the differences may appear small, these forms are retained as a distinct taxonomic unit because they have a distinct stratigraphic distribution with respect to $D$. calcaris. They have been recorded only in Zone NN6, whereas typical $D$. calcaris is not present in intervals underlying Zone NN9.

The distinction between $D$. signus (Plate 10, Figs. 4 and 7) and $D$. tuberi was impossible in most instances because the bifurcations are easily destroyed. Therefore, the two species have been lumped together in the range charts.

\section{Six-rayed Discoasterids with a Large Central Area}

In the middle Miocene, six-rayed discoasterids with a variable, broad central area and a diameter larger than the length of the rays are present. Rays can be blunt or have a slight bifurcation or a notch. Species circumscription within this group is difficult if the preservation state is not perfect. In the range charts, we have distinguished $D$. kugleri (Plate 6, Figs. 1 and 3 ) and $D$. musicus ( $=D$. sanmiguelensis) (Plate 11, Figs. 6a and 6b) on the basis of features of the central area, flat in the former and with a large star-shaped knob in the latter.

Recognition of $D$. kugleri, a marker species in the standard zonations, remains difficult. We have assigned to this species morphotypes similar to those illustrated by Bramlette and Wilcoxon (1967), and our species concept may have been too restricted. Many forms resembling $D$. kugleri are present below the level we have indicated as its FO at Hole 714A. 


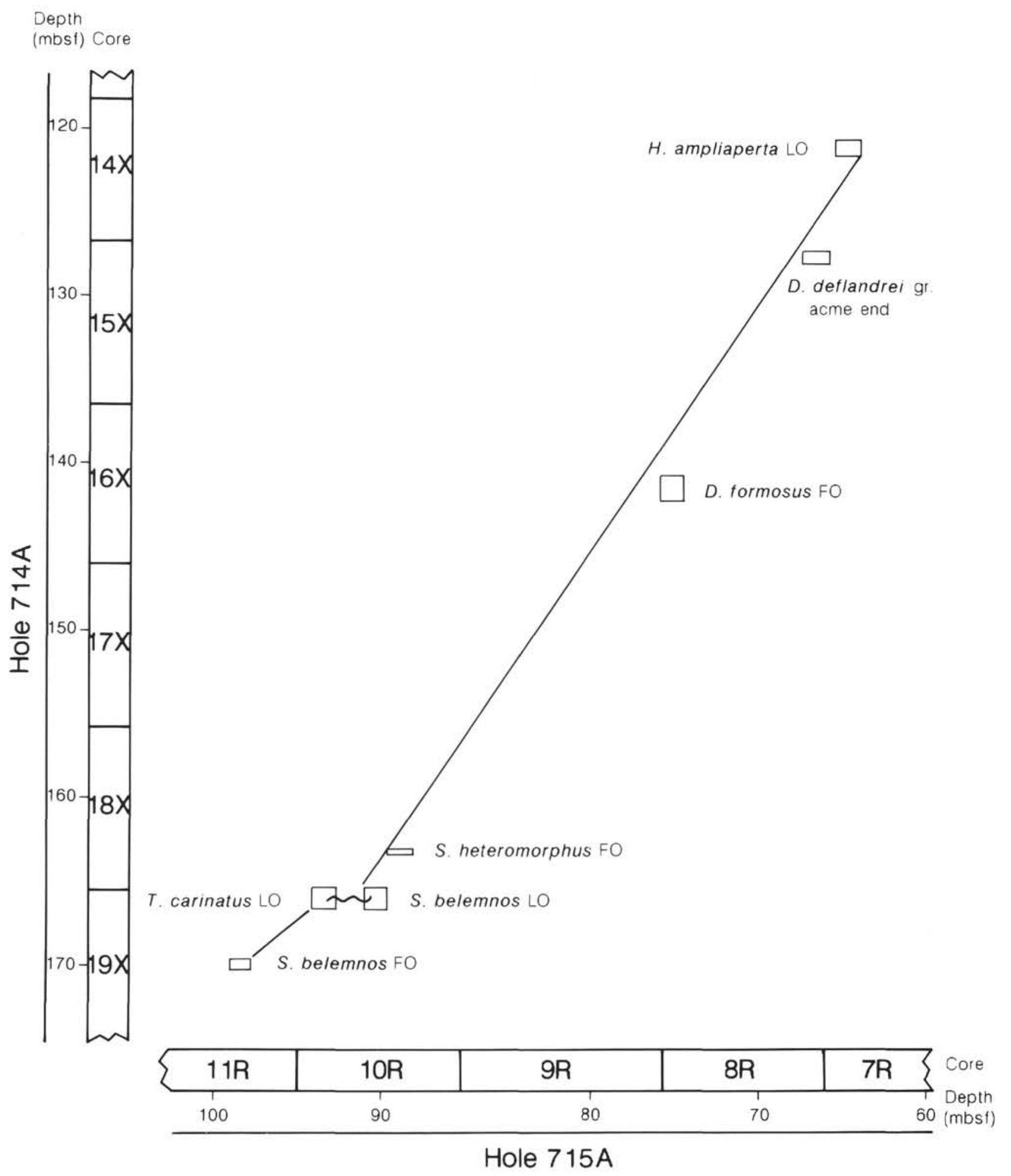

Figure 22. Shaw diagram for Holes 714A and 715A. 


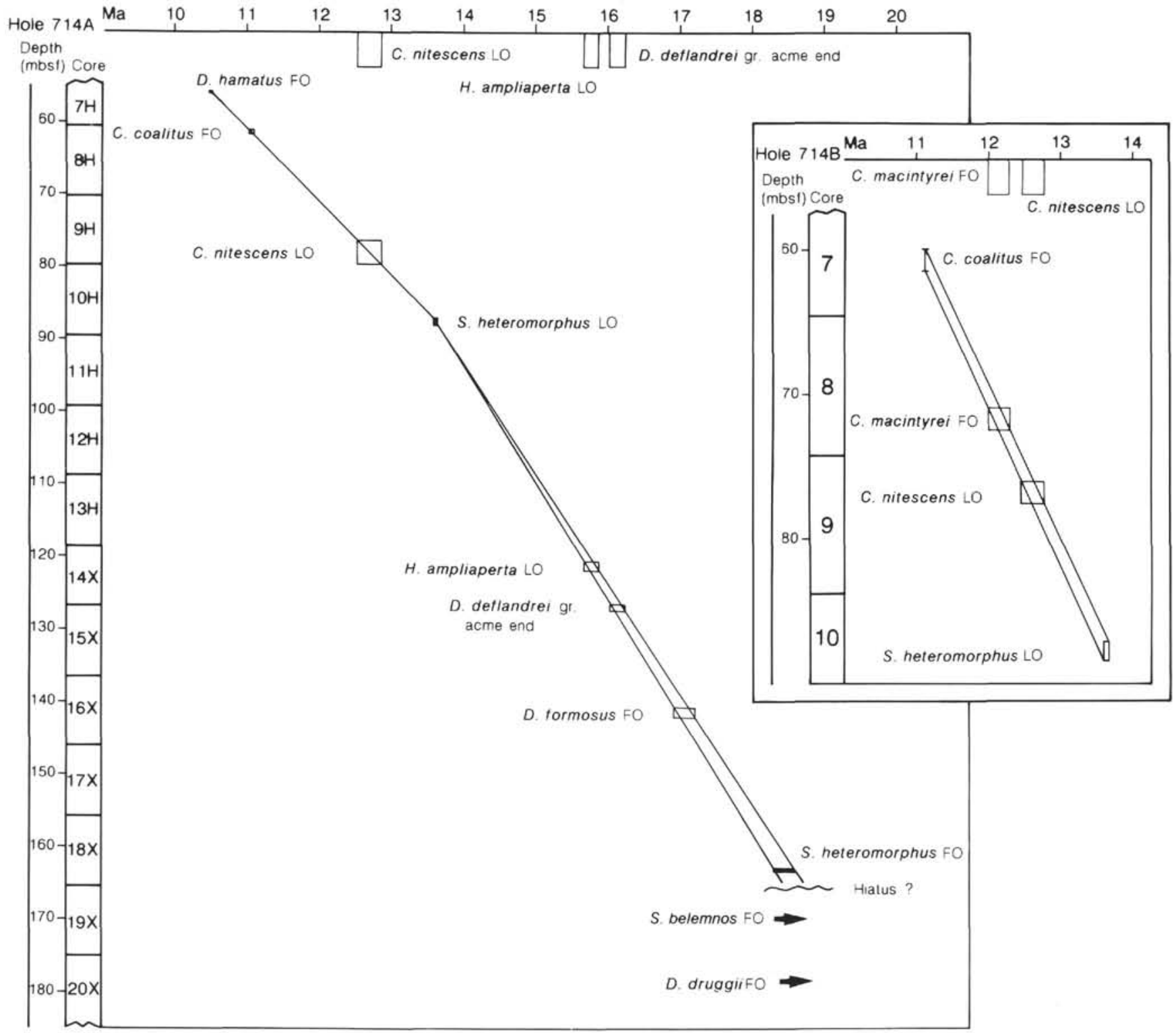

Figure 23. Age-depth plots of the early-middle Miocene sediments at Hole 714A and of middle Miocene sediments at Hole 714B based on nannofossil events calibrated at Site 710 .

\section{Discoaster adamanteus}

Small discoasterids with six rays, which are joined over most of their length, have been lumped together under this heading in the range charts. Because of the small size, they are also recognized in poorly preserved material.

\section{Discoaster aulakos}

This species is often placed within the $D$. deflandrei group, from which it is difficult to distinguish in badly preserved material. We have ascribed to this category discoasterids with six bifurcating rays that have a sharply pointed interarm area. It seems restricted to the advanced middle Miocene (Zone NN6).

\section{Early-Middle Miocene Discoasterids}

Early Miocene discoasterid assemblages are dominated by fairly massive, medium- to large-sized forms with short rays. Morphologic variability is large, but species that can be confi- dently recognized in poorly preserved material are few. We have adopted few large taxonomic units commented below.

Discoaster dilatus-D. extensus Group

We have included within this group discoasterids with slightly to broadly flaring rays (Plate 11 , Figs. 8 and 9). Distinction within this group (comprising $D$. extensus, D. phyllodus, and $D$. dilatus) proved impossible. The group is distributed from Zones NN1 to NN5, but it is more common in Zone NN4.

\section{Discoaster druggii and Discoaster formosus}

We have restricted the concept of $D$. druggii to asteroliths larger than $15 \mu \mathrm{m}$ in size with a flat central area. In our assemblages most of the morphotypes illustrated by Bramlette and Wilcoxon (1967) are present in some levels (Plate 10, Figs. 5, 6, 8 , and 9). Discoasterids with a flat central area, but smaller than $12 \mu \mathrm{m}$ in size, have been tabulated in the range charts as $D$. aff. druggii (Plate 11, Figs. 4 and 5). 


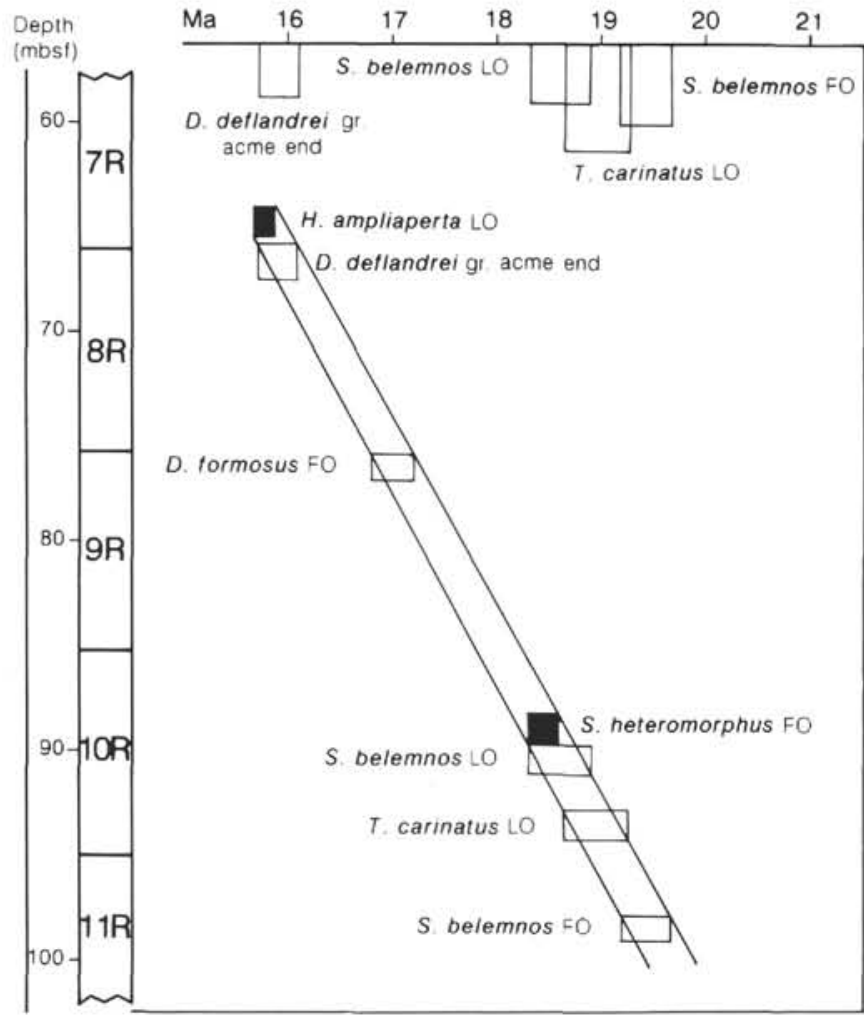

Figure 24. Age-depth plots of the early Miocene sediments at Hole 715A based on nannofossil events. Solid blocks represent control points.

Discoaster formosus is similar in size and variability to $D$. druggii, from which it is differentiated by the presence of a prominent knob in the central area (Plate 10, Figs. 1 and 2). Discoaster formosus appears in the middle of Zone NN4 (CN3) in the investigated sections, and its FO datum may be stratigraphically useful.

\section{Discoaster deflandrei Group}

A wide species concept has been followed for this taxonomic unit (Plate 10, Fig. 3), dominating the early and basal middle Miocene discoasterid assemblages.

\section{Discoaster woodringi Group}

$D$. woodringi is sometime considered an artificial preservational taxon (Roth and Thierstein, 1972). We have found discoidal discoasterids with six triangular rays (Plate 11, Figs. 1-3) in dissolved assemblages, and, therefore, we have retained these forms to be distinct from $D$. deflandrei group. These forms are distributed in the early Miocene and in the lower part of the middle Miocene.

\section{Gephyrocapsids}

It is generally held that in light microscope study the various species of Gephyrocapsa cannot be distinguished (Backman and Shackleton, 1983), and some (e.g., Bergen, 1984) state that because of the complex taxonomic interrelationship the gephyrocapsids cannot provide meaningful biostratigraphic information. However, Rio (1982), Rio et al. (in press a) have shown that by using plain size measurements of these placoliths it is possible to use this group consistently for biostratigraphic purposes. Williams et al. (1988) and Rio et al. (in press c) have shown that they can provide an accurate and precise tool for correlation in early Pleistocene sequences.

The biometrically based definitions of the gephyrocapsid group proposed by Rio et al. (in press a) have been followed here, splitting the group into four categories:

1. gephyrocapsids $<3.5 \mu \mathrm{m}$ in size, labeled "Small Gephyrocapsa";

2. gephyrocapsids $>4 \mu \mathrm{m}$ and $<5.5 \mu \mathrm{m}$ in size, with an open central area, labeled Gephyrocapsa oceanica s.l.;

3. gephyrocapsids $>5.5 \mu \mathrm{m}$ in size labeled "Large Gephyrocapsa"; and

4. gephyrocapsids usually 4-6 $\mu \mathrm{m}$ in size with an open central area and a bridge nearly aligned with the short axis of the placolith, labeled Gephyrocapsa sp. 3. They are comparable to Gephyrocapsa parallela, as figured by Takayama and Sato (1985), and are similar to $G$. oceanica, as intended by many authors who attach significance to the bridge orientation for the definitions of gephyrocapsids at the specific level. For illustrations of these gephyrocapsid groups, see Rio (1982).

\section{Reticulofenestrids}

\section{Reticulofenestra pseudoumbilicus}

The distinction between $R$. pseudoumbilicus and the other members of the group is based on the overall size of the placo-

Table 30. Summary of the position of calcareous nannofossil events at Hole 716B.

\begin{tabular}{|c|c|c|}
\hline Event & $\begin{array}{l}\text { Core, section, } \\
\text { interval }(\mathrm{cm})\end{array}$ & $\begin{array}{l}\text { Depth } \\
\text { (mbsf) }\end{array}$ \\
\hline Gephyrocapsa spp. $>5.5 \mu \mathrm{m}$ FO & $115-716 \mathrm{~B}-5 \mathrm{H}-3,50 / 115-716 \mathrm{~B}-5 \mathrm{H}-2,50$ & $36.3-34.8$ \\
\hline C. macintyrei LO & $115-716 \mathrm{~B}-6 \mathrm{H}-3,50 / 115-716 \mathrm{~B}-6 \mathrm{H}-1,50$ & $46.0-43.0$ \\
\hline G. oceanica FO & $115-716 \mathrm{~B}-7 \mathrm{H}-3,50 / 115-716 \mathrm{~B}-7 \mathrm{H}-2,50$ & $55.6-54.1$ \\
\hline D. brouweri LO & $115-716 \mathrm{~B}-8 \mathrm{H}-2,50 / 115-716 \mathrm{~B}-8 \mathrm{H}-1,50$ & $63.7-62.2$ \\
\hline D. pentaradiatus LO & $115-716 \mathrm{~B}-9 \mathrm{H}-2,50 / 115-716 \mathrm{~B}-9 \mathrm{H}-1,50$ & $72.9-71.9$ \\
\hline D. surculus LO & ND & \\
\hline D. tamails LO & ND & \\
\hline Sphenoliths LO & $115-716 \mathrm{~B}-12 \mathrm{H}-4,50 / 115-716 \mathrm{~B}-12 \mathrm{H}-3,50$ & $105.3-103.8$ \\
\hline R. pseudoumbilica LO & $115-716 \mathrm{~B}-15 \mathrm{H}-2,50 / 115-716 \mathrm{~B}-15 \mathrm{H}-1,50$ & $131.3-129.4$ \\
\hline Amaurolithus spp. LO & ND & \\
\hline C. rugosus $\mathrm{FO}$ & $115-716 \mathrm{~B}-14 \mathrm{H}-7,50 / 115-716 \mathrm{~B}-14 \mathrm{H}-3,50$ & $129.2-123.2$ \\
\hline C. acutus FO & ND & \\
\hline T. rugosus LO & ND & \\
\hline D. quinqueramus LO & $115-716 \mathrm{~B}-17 \mathrm{H}-1,50 / 115-716 \mathrm{~B}-16 \mathrm{H}-7,50$ & $149.1-148.4$ \\
\hline Amaurolithus spp. FO & $115-716 \mathrm{~B}-26 \mathrm{H}-3,50 / 115-716 \mathrm{~B}-26 \mathrm{H}-2,50$ & $240.5-239.0$ \\
\hline
\end{tabular}

Note: $\mathrm{LO}=$ last occurrence, $\mathrm{FO}=$ first occurrence, and ND $=$ not detected. 
lith following Raffi and Rio (1979) and Backman and Shackleton (1983). Reticulofenestrids larger than $7 \mu \mathrm{m}$ are classified as $R$. pseudoumbilicus. Given this definition we have found that members of this morphogroup appear in the area at the boundary between Zones NN5 (CN4) and NN6 (CN5) in all the investigated sections. After appearing with forms around $7 \mu \mathrm{m}$ in size, the group undergoes a size increase, and forms as large as $12 \mu \mathrm{m}$ have been observed in the NN6-NN7 zonal interval. As commented in the biostratigraphic section, the group virtually disappears from the stratigraphic record for a long interval in the late Miocene. The Pliocene extinction datum of large $R$. pseudoumbilicus is quite distinct in the Leg 115 material except in Maldives Site 716. This event occurs in the late Gilbert Chron as it does in other areas (Backman and Shackleton, 1983; Rio et al., 1989).

Reticulofenestra gelida, considered by Backman (1980) to be a cold-water ecovariant of $R$. pseudoumbilicus, is present in the material studied, but it has not been tabulated in the range charts.

\section{Reticulofenestra spp.}

Reticulofenestrids with a morphology similar to $R$. pseudoumbilicus but of a smaller size have been lumped under the heading "Reticulofenestra spp." in the range charts. This group includes species like $R$. haqii, $R$. minuta, and $R$. minutula.

\section{Reticulofenestra daviesi}

This species was recognized by the presence of a thick central reticulate plug (Plate 14, Fig. 5). It is sometimes ascribed to the genus Dictyococcites (i.e. Perch-Nielsen, 1985). Intergrade forms to $R$. lockeri, which is characterized by a bridgelike structure in the central area, have been noted. This group is restricted to the early Miocene, where it represents a subordinate component of the assemblage.

\section{Reticulofenestra aff. pseudoumbilicus}

In the early Miocene reticulofenestrids larger than $7 \mu \mathrm{m}$ in size that are similar to $R$. pseudoumbilicus are present. They are characterized, however, by the presence of small "teeth", barely visible in the light microscope, in the margin of the central area (Plate 14, Figs. 10a and 10b). These forms become extinct in the early Miocene, and their distribution does not overlap with that of $R$. pseudoumbilicus.

\section{Calcidiscus}

Calcidiscus macintyrei may be confused with different varieties of Calcidiscus leptoporus. Backman and Shackleton (1983) proposed the overall placolith size as a practical means to separate these taxa in the light microscope. Since measurements of late Pliocene and early Pleistocene populations of the Calcidiscus group indicate a bimodal distribution of size (see Rio et al., in press b), they proposed $10 \mu \mathrm{m}$ as the critical size to make the distinction between these two species. This proposal has been followed here, and only circular Calcidiscus equal to or larger than $11 \mu \mathrm{m}$ have been labeled $C$. macintyrei in this work.

Following this concept, the first appearance of $C$. macintyrei occurs at a higher stratigraphic level than that indicated by Bukry (1978). It should be noted, however, that biometric measurements (Fornaciari et al., this volume) in middle Miocene circular Calcidiscus populations do not indicate a size bimodal distribution like that detected in the late Pliocene-early Pleistocene populations. Calcidiscus that are $10 \mu \mathrm{m}$ in size are abundant, whereas they are rare and/or missing in the early Pleistocene populations (Fornaciari et al., this volume).

Rare elliptical Calcidiscus (Plate 14, Figs. 4a and 4b) are present in Zones NN4 and NN5, which have been referred to Calcidiscus premacintyrei.

\section{Sphenoliths}

Sphenoliths are a major constituent of the Miocene and early Pliocene nannofossil assemblages of the western equatorial Indian Ocean and provide several important biohorizons. An effort has been made in this work and in the companion paper of Fornaciari et al. (this volume) to establish the ranges of the most important taxa. Other taxa, of difficult recognition or unimportant stratigraphically, have been lumped together in the range charts. Taxonomic criteria and procedures followed in recognizing critical species are clarified below.

\section{Sphenolithus spp.}

Under this heading in the range chart, we have lumped together several intergrading but distinct species like $S$. moriformis, $S$. compactus, $S$. abies, and $S$. neoabies. Distinctions among these taxa are not always straightforward and seemed unnecessary because the only useful bioevent is provided by the simultaneous (Backman and Shackleton, 1983) extinction of S. abies and $S$. neoabies in the Pliocene.

\section{Sphenolithus belemnos}

The species has been identified, adhering strictly to the original description of Bramlette and Wilcoxon (1967), by the dartshaped outline in side view, by the high proximal column, and by the presence of an apical spine at $45^{\circ}$ at crossed nicols, which disappears when viewed at $0^{\circ}$ (Plate 12, Figs. $3 \mathrm{~b}$ and $3 \mathrm{c}$ ). The pattern of extinction at crossed nicols at about $20^{\circ}$ is also useful in recognizing the species (Plate 12, Fig. 3a). The range of $S$. belemnos is well documented only in Sites 714 and 715 , because a hiatus is present at all other early Miocene sequences examined. Our results indicate that it disappears virtually at the level of appearance of typical $S$. heteromorphus.

\section{Sphenolithus calyculus}

This small-spined sphenolith was recognized, following Bukry (1985), on the basis of the "calyx" present at the base of the apical spine and on the basis of the relative size of the quadrants characterizing the base when viewed at crossed nicols with the major axis aligned to a polarization direction. The species is not always recognized confidently, and its distribution is difficult to pinpoint precisely. It has been detected in Zones NN1 and NN2.

\section{Sphenolithus capricornutus}

The species is easily recognized by the presence of two prominent, tapering, diverging spines unique to this species (Plate 12, Figs. $5 \mathrm{a}-5 \mathrm{c})$. It is often reported as distributed from the latest Oligocene to the early Miocene (Perch-Nielsen, 1985). In the material examined it is restricted to the upper part of Zone NN1, being codistributed with $S$. delphix.

\section{Sphenolithus conicus}

Sphenolithus conicus was recognized by its large size, its triangular outline and its complex apical spine (Plate 12, Figs. 4a and $4 \mathrm{~b}, 6 \mathrm{a}$ and $6 \mathrm{~b}$ ). We did not meet with special problems in differentiating this form from $S$. heteromorphus. In the equatorial Indian Ocean, the species appears in the late Oligocene and disappears close to the base of Zone NN4 (see Fornaciari et al., this volume).

\section{Sphenolithus delphix}

The species is easily recognized by the triradiate outline in side view (Plate 11, Figs. 10a-10c, 11a, 11b, and 12), deriving from the expansion of the basal elements of the basal shield. The species is reported in the literature as appearing in the late Oligocene (Perch-Nielsen, 1985). In the western equatorial Indian Ocean, it appears in the early Miocene Zone NN1, under- 
going an acme just below the FO of $D$. druggii. This acme interval is one of the most prominent biostratigraphic features of the early Miocene in all the sequences examined, and it is useful to monitor the top part of Zone NN1.

\section{Sphenolithus dissimilis}

This small form has been recognized by the three-part arrangement of its apical spine when viewed at $45^{\circ}$ at crossed nicols (Plate 12, Figs. 7 and 8). Another feature of the species, easily observed in well-preserved material, is the optical bipartition of the basal part (Bukry and Percival, 1971). While typical specimens are easily recognized, many intergrade forms exist to other small undescribed early Miocene sphenoliths and to S. belemnos, as commented below.

The appearance of the species occurs in the late Oligocene, and it apparently becomes extinct in the early Miocene within basal Zone NN4.

\section{Sphenolithus dissimilis-Sphenolithus belemnos Intergrade}

In the early Miocene we observed sphenoliths with a dartshaped outline, similar to that of $S$. belemnos, when viewed at crossed nicols at $0^{\circ}$, and with a tripartite apical part, similar to that of $S$. dissimilis, when viewed at crossed nicols at $45^{\circ}$. These forms (Plate 12, Figs. 2a-2c) have been tabulated in the range charts as $S$. dissimilis-S. belemnos intergrade. They are common in the basal part of Zone NN2 (e.g. Hole 710A), and only a few specimens were found within the range of $S$. belemnos.

\section{Sphenolithus pseudoheteromorphus, Sphenolithus milanettii, and Sphenolithus multispinatus}

These species have been recently described in material from Leg 115 by Rio et al. (in press b), to which the reader is referred. For illustrations, see Fornaciari et al. (this volume).

\section{Ceratolithids}

These horseshoe-shaped members of the Ceratolithaceae family are an indispensable means for the biostratigraphic subdivision of the latest Miocene and early Pliocene. They are a minor component of the assemblages (rarely they exceeding $1 \%$ of the total flora). The variable preservation observed in the CBT sites provide information on the impact of preservation on their classification. The beautifully preserved assemblages, found at several sites, may be useful to clarify the phylogenetic relationship of the group. The stratigraphic succession of events based on ceratoliths is from bottom to top:
1. FO of $A$. primus,
2. FO of $A$. delicatus,
3. FO of $A$. amplificus,
4. LO of A. amplificus,
5. FO of $C$. acutus,
6. FO of A. tricorniculatus,
7. FO of C. armatus,
8. FO of C. rugosus,
9. LO of C. acutus,
10. LO of C. armatus, and
11. LO of $A$. delicatus and A. tricorniculatus.

A wide variability was adopted in tabulating the range of Amaurolithus primus. The earlier forms of the group are represented by specimens with a very thick arch or an apical lump (Plate 7, Figs. 1 and 2), which closely compare with the holotype (see Bukry and Percival, 1971, Plate 1, fig. 12). These primitive forms become extinct in the late Miocene, whereas more evolved crescent-shaped forms (Plate 7, Figs. 3 and 4), be- come extinct in the early Pliocene. A distinction between these two morphotypes may prove to be stratigraphically useful.

Simple, delicate, horseshoe-shaped ceratolithids, sometimes possessing a small apical spur (Plate 7, Figs. 6-8), have been ascribed to Amaurolithus delicatus.

Robust, asymmetrical ceratolithids with two large horns, one of which bears a row of nodes, were ascribed to Amaurolithus amplificus (Plate 8, Figs. 7, 9, and 11-14). The form is recognized also in overgrown assemblages because of its large size and "angular" aspect. Intergrade forms between Triquetrorhabdulus extensus and A. amplificus were observed (Plate 8, Figs. 4-6, 8, and 10), thus confirming previous suggestions of a phylogenetic relationship between Triquetrorhabdulus and Amaurolithus (Perch-Nielsen, 1977, 1985). These transitional forms were found within and below the range of typical A. amplificus.

Slightly asymmetrical ceratolithids, with a delicate construction and a pronounced apical spine, were ascribed to $A$. tricorniculatus (Plate 7, Figs. 11-13). The species is seemingly restricted to early Pliocene sediments in the investigated sections. Other authors (e.g., Gartner and Bukry, 1975; Theodoridis, 1984) reported $A$. tricorniculatus as rare in late Miocene sediments. In this time interval we found only forms with a small apical spur (Plate 7, Figs. 5 and 9) which were considered as intergrade forms and/or morphotypes of $A$. delicatus.

Among the birefringent ceratolithids, we recognized $C$. acutus, C. armatus, C. rugosus, C. cristatus, and C. telesmus (Plate 9). Specimens comparable to the respective holotypes and specimens transitional between the different species are present, and species assignments are sometimes difficult. Overgrowth has been a further drawback in classifying ceratolithids, especially at Site 716 . In the range charts, abundance data refer only to specimens confidently recognized by comparison to respective holotypes. Only $C$. cristatus has been used in a wide sense, inclusive of intergrade forms to $C$. rugosus and $C$. telesmus.

Recently, Bergen (1984) found a concomitant range of $C$. rugosus and $C$. cristatus and suggested that they are conspecific, considering their different morphologies related to different preservation states. In Leg 115 sections, indicative of varied preservation states, typical $C$. rugosus appears below $C$. cristatus. Intergrade forms between the two forms are present only well above the level we consider to be the FO of $C$. rugosus. Therefore, we retain the two species to be distinct, even if preservation may obscure diagnostic features in heavily overgrown and dissolved assemblages. C. telesmus occurs in our material, but has not been tabulated in the range charts.

\section{Triquetrorhabdulids}

Species ascribed to the genus Triquetrorhabdulus make up a significant part of the Miocene calcareous nannofossil assemblages in the equatorial Indian Ocean, and provide useful biostratigraphic events in the Miocene. All the species described in the literature (compare with Aubry, 1988) were easily identified, except $T$. striatus.

Triquetrorhabdulus challengeri (Plate 13, Figs. $3 a$ and $3 b$ ) is very rare and sporadic in early Miocene sediments. We have followed Olafsson (1989) in ascribing Orthorhabdus serratus to the genus Triquetrorhabdulus. Forms morphologically indistinguishable from $T$. serratus which are nonbirefringent (Plate 13, Figs. 4 and 7) were also observed. We realized their presence only at the end of this work; therefore, they were tabulated only in some range charts as $T$. cf. serratus.

Triquetrorhabdulus backmanii, T. curvus, and T. protodecimae were recently described from material of Leg 115 by Rio et al. (in press b), to whom the reader is referred for description 
and illustrations. They are subordinate forms and have restricted stratigraphic ranges.

Triquetrorhabdulus rugosus (Plate 7, Figs. 14 and 15) and $T$. rioensis have a similar stratigraphic distribution and were lumped together in the range charts. Triquetrorhabdulus rugosus shows a large morphologic variability, and $T$. farnsworthii, characterized by a curved ridge, was not considered as a distinct species.

\section{Helicoliths}

Helicoliths occur continuously only in the Maldives area, whereas they are intermittently present in the other sites. This contrasting distribution pattern is typical for the group (PerchNielsen, 1985), which occurs most commonly and continuously in hemipelagic sediments and seems to prefer water masses with high nutrient contents. The group is also solution prone, and this fact may explain its absence in many oceanic sediments.

Perch-Nielsen (1985) pointed out that the classification of Helicosphaera species using light microscopy is difficult, and the species concepts adopted are based strictly on the holotype. Little variability was assumed but for $H$. carteri, which is used in a broad sense.

We have identified genuine $H$. recta (Plate 14, Figs. 8 and 9) in Zone NN4 at Sites 714 and 715 . There is no reworking in this interval at these sites; therefore, the finding indicates a more extended range for this species than previously believed (PerchNielsen, 1985).

Helicosphaera perch-nielsinae is well represented in the Miocene and becomes extinct in Zone NN5. Perch-Nielsen (1985) reported the LO of this form in Zone NP24. Müller (1981) considers this species as becoming extinct in Zone NN6. Helicosphaera obliqua is recorded as scattered up to Zone NN5. PerchNielsen (1985) reports the LO of this form at the top of Zone NN4. Müller (1981) reports the LO of this species in Zone NN6. Helicosphaera mediterranea (junior synonym $H$. crouchi) was found to be rare in the early Miocene Zones NN2, NN3, and $\mathrm{NN} 4$, in agreement with previous distributions indicated by Perch-Nielsen (1985). According to Müller (1981) the species is restricted to Zones NN2 and NN3. Helicosphaera euphratis has been found as high as Zone NN6, in agreement with the previous suggestions of Perch-Nielsen (1985) and Müller (1981). Helicosphaera intermedia is present at Site 714 up to Zone NN7. Perch-Nielsen (1985) reports the LO of this species in Zone NN5.

\section{Lopadolith Scyphospherids}

No attempt was made to determine this highly diversified group at the species level, which occurs consistently in the Maldives area. Martini and Worsley (1971) noted that its stratigraphic distribution in the western Pacific suggests that this group is strongly influenced by regional ecological conditions. Scyphospherids enter the stratigraphic record of the western equatorial Indian Ocean during the middle Miocene (upper part of Zone NN4 in Sites 714 and 715).

\section{Pontospherids}

The pontospherids form a subordinate part of the nannofossil assemblages in Leg 115 material. They are not resistant to dissolution (Bergen, 1984) and are generally not used for biostratigraphic classification. They are tabulated only at a generic level in the range charts.

\section{ACKNOWLEDGMENTS}

The constructive criticism provided by J. Backman (Stockholm), K. Perch-Nielsen (Zurich), and W. Wei (Tallahassee) is greatly appreciated. Special thanks go to C. Brogiato (Padova), A. Negri (Bologna), A. Haidar (Parma), and G. Villa (Parma) for assisting in various phases of this work. Funding for this study was provided by CNR Grant 88.01644 .05 to D. Rio.

\section{REFERENCES}

Adelseck, C. G., Geehan, G. W., and Roth, P. H., 1973. Experimental evidence for selective dissolution and overgrowth of calcareous nannofossils during diagenesis. Bull. Geol. Soc. Am., 84:2755-2762.

Aguirre, E., and Pasini, G., 1985. The Pliocene/Pleistocene boundary. Episodes, 8:11-120.

Aubry, M. P., 1988. Handbook of Cenozoic Calcareous Nannoplankton. Book 2: Ortholithae (Catinasters, Ceratoliths, Rhabdoliths): New York (Micropaleontology Press).

Backman, J., 1980. Miocene-Pliocene nannofossils and sedimentation rates in the Hatton-Rockall Basin, northeast Atlantic Ocean. Stockholm Contrib. Geol., 36(1):1-91.

Backman, J., Duncan, R., et al., 1988. Proc. ODP, Init. Repts., 115: College Station, TX (Ocean Drilling Program).

Backman, J., and Pestiaux, P., 1986. Pliocene Discoaster abundance variations, Deep Sea Drilling Project Site 606: biochronology and paleoenviromental implications. In Ruddiman, W. F., Kidd, R. B., Thomas, E., et al., Init. Repts. DSDP, 94, Pt. 2: Washington (U.S. Govt. Printing Office), 903-910.

Backman, J., and Shackleton, N. J., 1983. Quantitative biochronology of Pliocene and early Pleistocene calcareous nannoplankton from the Atlantic, Indian, and Pacific oceans. Mar. Micropaleontol., 8: 141-170.

Barron, J. A., Keller, G., and Dunn, D. A., 1985. A multiple microfossil biochronology for the Miocene. In Kenneth, J. P. (Ed.), The Miocene Ocean: Paleoceanography and Biogeography. Mem. Geol. Soc. Am., 163:21-36.

Bergen, J. A., 1984. Calcareous nannoplankton from Deep Sea Drilling Project Leg 78A: evidence for imbricate underthrusting at the lesser Antillian active margin. In Biju-Duval, B., Moore, J. C., et al., Init. Repts. DSDP, 78A: Washington (U.S. Govt. Printing Office), 411446.

Berger, W. H., 1978. Sedimentation of deep-sea carbonate: maps and models of variations and fluctuations. J. Foraminiferal Res., 8:286302.

Berggren, W. A., Kent, D. V., and Van Couvering, J. A., 1985. The Neogene: Part 2. Neogene geochronology and chronostratigraphy. In Snelling, N. J. (Ed.), The Chronology of the Geological Record. Mem. Geol. Soc. (London), 211-260.

Berggren, W. A., and Van Couvering, J. A., 1974. The late Neogene. Palaeogeogr., Palaeoclimatol., Palaeoecol., 16:1-215.

Bizon, G., and Müller, C., 1979. Remarks on the Oligocene/Miocene boundary based on the results obtained from the Pacific and the Indian Oceans. VII Int. Congr. Medit. Neogene, Ann. Geol. Pays Hell., 1:101-111.

Bode, G. W., and Cronin, D. S., 1973. Carbon and carbonate analysis, Leg 16. In van Andel, T. H., Heath, G. R., et al., Init. Repts. DSDP, 16: Washington (U.S. Govt. Printing Office), 521-528.

Bramlette, M. N., and Wilcoxon, J. A., 1967. Middle Tertiary calcareous nannoplankton of the Cipero Section, Trinidad, B.W.I. Tulane Stud. Geol., 5:93-132.

Broecker, W. S., 1981. Glacial to interglacial changes in ocean and atmosphere chemistry. In Berger, E. (Ed.), Climatic Variations and Variability: Facts and Theories: Hingham, MA (D. Reidel), 109-120. 1982. Glacial to interglacial changes in ocean chemistry. Prog. Oceanogr., 2:151-197.

Bukry, D., 1971. Discoaster evolutionary trends. Micropaleontology, 17:43-52.

1972. Coccolith stratigraphy-Leg 14, Deep Sea Drilling Project. In Hayes, D. E., Pimm, A. C., et al., Init. Repts. DSDP, 14: Washington (U.S. Govt. Printing Office), 487-494.

1973. Low-latitude coccolith biostratigraphic zonation. In Edgard, N. T., Saunders, J. B., et al., Init. Repts. DSDP, 15: Washington (U.S. Govt. Printing Office), 685-703.

1975. Coccolith and silicoflagellate stratigraphy, northwestern Pacific Ocean Deep Sea Drilling Project, Leg 32. In Larson, R. L., Moberly, R., et al., Init. Repts. DSDP, 32: Washington (U.S. Govt. Printing), 677-701.

1978. Biostratigraphy of Cenozoic sediment by calcareous nannofossils. Micropaleontology, 24:44-60. 
1985. Mid-Atlantic Ridge coccolith and silicoflagellate biostratigraphy, Deep Sea Drilling Project Sites 558 and 563. In Bougault, H., Cande, S. C., et al., Init. Repts. DSDP, 82: Washington (U.S. Govt. Printing), 591-603.

Bukry, D., and Bramlette, M. N., 1969. Some new and stratigraphically useful calcareous nannofossils of the Cenozoic. Tulane Stud. Geol., 7:131-142.

Bukry, D., and Percival, S. F., 1971. New tertiary calcareous nannofossils. Tulane Stud. Geol., 8:123-146.

Channell, J.E.T., Rio, D., Sprovieri, R., and Glacon, G., 1989. Biomagnetostratigraphic correlations from Leg 107 in the Tyrrenian Sea. In Kastens, K., Mascle, J., et al., Proc. ODP, Sci. Results, 107: College Station, TX (Ocean Drilling Program), 669-682.

Channell, J.E.T., Rio, D., and Thunell, R. C., 1988. Miocene/Pliocene boundary magnetostratigraphy at Capo Spartivento (Calabria, Italy). Geology, 16:1096-1099.

Cita, M. B., 1975. The Miocene/Pliocene boundary: history and definition. In Saito, T., and Burkle, L. H. (Eds.), Late Neogene Epoch Boundaries: New York (Micropaleontology Press), 1-30.

Cita, M. B., and Premoli Silva, I., 1968. Evolution of the planktonic foraminiferal assemblages in the stratigraphical interval between the type-Langhian and the type-Tortonian and biozonation of the Miocene of Piedmont. G. Geol., 35:1-27.

Cita, M. B., Premoli Silva, I., and Rossi, R., 1965. Foraminiferi planctonici del Tortoniano-tipo. Riv. It. Paleontol., 71:217-308

Droxler, A. W., Schlager, W., and Whallon, C. C., 1983. Quaternary aragonite cycles and oxygen-isotope record in Bahamian carbonate ooze. Geology, 11:235-239.

Dunn, D. A., 1982. Miocene sediments of the equatorial Pacific Ocean: carbonate stratigraphy and dissolution history (Ph.D. dissert.). Univ. Rhode Island, Kingston.

Ellis, H. C., 1981. Calcareous nannoplankton biostratigraphy-Deep Sea Drilling Project Leg 60. In Hussong, D. M., Uyeda, S., et al., Init. Repts. DSDP, 60: Washington (U.S. Govt. Printing Office), 507-533.

Forbes, E., 1846. On the connection between the distribution of the existing fauna and flora of the British Isles and the geographical changes which have affected their area, especially during the epoch of the Northern Drift. G.B. Geol. Survey, Mem., 1:336-432.

Gartner, S., 1974. Nannofossil biostratigraphy, Leg 22, Deep Sea Drilling Project. In von der Borch, C. C., Sclater, J. G., et al., Init. Repts. DSDP, 22: Washington (U.S. Govt. Printing Office), 577599.

Gartner, S., and Bukry, D., 1975. Morphology and phylogeny of the coccolithophycean family Ceratholitaceae. J. Res. U.S. Geol. Surv., 3:451-465.

Gartner, S., and Chow, J., 1985. Calcareous nannofossil biostratigraphy, Deep Sea Drilling Project Leg 85, eastern equatorial Pacific. In Mayer, L., Theyer, F., Thomas, E., et al., Init. Repts. DSDP, 85: Washington (U.S. Govt. Printing Office), 609-619.

Gartner, S., Chow, J., and Stanton, R. J., 1987. Late Neogene paleoceanography of the eastern Caribbean, the Gulf of Mexico, and the eastern equatorial Pacific. Mar. Micropaleontol., 12:255-304.

Haq, B. U., 1980. Biogeographic history of Miocene calcareous nannoplankton and paleoceanography of the Atlantic Ocean. Micropaleontology, 26:414-443.

Haq, B. U., Hardenbol, J., and Vail, P. R., 1987. Chronology of fluctuating sea levels since the Triassic. Science, 235:1156-1167.

Hekel, H., 1973. Late Oligocene to Recent Nannoplankton from the Capricorn Basin (Great Barrier Reef Area). Publ. Geol. Surv. Queensl., 359, Palaeontol. Pap., 33:1-24.

Jiang, M. J., and Gartner, S., 1984. Neogene and Quaternary calcareous nannofossil biostratigraphy of the Walvis Ridge. In Moore, T. C., Jr., Rabinowitz, P. D., et al., Init. Repts. DSDP, 74: Washington (U.S. Govt. Printing Office), 561-595.

Keigwin, L. D., 1982. Stable isotope stratigraphy and paleoceanography of Sites 502 and 503. In Prell, W. L., Gardner, J. V., et al., Init. Repts. DSDP, 68: Washington (U.S. Govt. Printing Office), 445453.

Keigwin, L. D., 1987. Pliocene stable isotope record of DSDP Site 606: sequential events of ${ }^{18} \mathrm{O}$ enrichment beginning at 3.1 Ma. In Ruddiman, W. F., Kidd, R. B., Thomas, E., et al., Init. Repts. DSDP, 94, Pt. 2: Washington (U.S. Govt. Printing Office), 911-920.
Keller, G., and Barron, J. A., 1987. Paleodepth distribution of Neogene deep sea hiatuses. Paleoceanography, 2:697-713.

La Brecque, J. L., Kent, D. V., and Cande, S. C., 1977. Revised magnetic polarity time scale for the Cretaceous and Cenozoic. Geology, 5:330-335.

Loeblich, A. R., and Tappan, H., 1966. Annotated index and bibliography of the calcareous nannoplankton. Phycologia, 5:81-216. 1968. Annotated index and bibliography of the calcareous nannoplankton II. J. Paleontol., 42:584-598. 1969. Annotated index and bibliography of the calcareous nannoplankton III. J. Paleontol., 43:568-588. , 1970a. Annotated index and bibliography of the calcareous nannoplankton IV. J. Paleontol., 44:558-574. 1970b. Annotated index and bibliography of the calcareous nannoplankton V. Phycologia, 9:157-174. 1971. Annotated index and bibliography of the calcareous nannoplankton VI. Phycologia, 10:315-339.

1973. Annotated index and bibliography of the calcareous nannoplankton VII. J. Paleontol., 47:715-759.

MacKenzie, D. T., and Wise, S. W., Jr., 1983. Paleocene and Eocene calcareous nannofossils from Deep Sea Drilling Project Legs 25 and 40 , south and east of Africa. In Ludwig, W. J., Krasheninnikov, V. A., et al., Init. Repts. DSDP, 71: Washington (U.S. Govt. Printing Office), 1141-1169.

Martini, E., 1971. Standard Tertiary and Quaternary calcareous nannoplankton Zonation. In Farinacci, A. (Ed.), Proceedings of the Second International Conference on Planktonic Microfossils, Roma: Rome (Tecnoscienza), 739-785.

1975. Calcareous nannoplankton from the type Tortonian (Upper Miocene). VI Congr. Reg. Comm. Medit. Neog. Strat. Bratislava, 1975, 53-56.

Martini, E., and Worsley, T., 1971. Tertiary calcareous nannoplankton from the western equatorial Pacific. In Winterer, E. L., Riedel, W. R., et al., Init. Repts. DSDP, 7, Pt. 2: Washington (U.S. Govt. Printing Office), 1471-1507.

Mayer, L. A., Shipley, T. H., and Winterer, E. L., 1986. Equatorial Pacific seismic reflectors as indicators of global oceanographic events. Science, 233:761-764.

Mazzei, R., Raffi, I., Rio, D., Hamilton, N., and Cita, M. B., 1979. Calibration of the late Neogene calcareous plankton datum planes with the paleomagnetic record of Site 397 and correlation with Moroccan and Mediterranean sections. In von Rad, U., Ryan, W.B.F., et al., Init. Repts. DSDP, 47: Washington (U.S. Govt. Printing Office), 375-389.

Miller, K. G., Fairbanks, R. G., and Mountain, G. S., 1987. Tertiary oxygen isotope synthesis, sea level history, and continental margin erosion. Paleoceanography, 2:1-19.

Müller, C., 1970. Nannoplankton-zonen der unteren Meeresmolasse Bayerns. Geol. Bavar., 63:107-118.

1974. Calcareous nannoplankton, Leg 25 (western Indian Ocean). In Simpson, E.S.W., Schlich, R., et al., Init. Repts. DSDP, 25: Washington (U.S. Govt. Printing Office), 579-633.

1977. Distribution of calcareous nannoplankton in Oligocene to Holocene sediments of the Red Sea and Indian Ocean reflecting paleoenviroment. In Heirtzler, J. R., Bolli, H. M., Davies, T. A., Saunders, J. B., Sclater, J. G., Indian Ocean Geology and Biostratigraphy: Washington (American Geophysical Union), 409422.

1981. Beschreibung neuer Helicosphaera-arten aus dem Miozn und revision biostratigraphischer reichweiten einiger Neogenen nannoplankton-arten. Senck. Leth., 61:427-435.

Muza, J. P., Wise, S. W., Jr., and Covington, J. M., 1986. Neogene calcareous nannofossils from Deep Sea Drilling Project Site 603, Lower Continental Rise, western North Atlantic: biostratigraphy and correlations with magnetic and seismic stratigraphy. In van Hinte, J. E., Wise, S. W., Jr., et al., Init. Repts. DSDP, 93: Washington (U.S. Govt. Printing Office), 593-616.

Okada, H., and Bukry, D., 1980. Supplementary modification and introduction of code numbers to the low-latitude coccolith biostratigraphic zonation (Bukiv. 19*3;1975). Mar. Micropaleontol., 5:321325.

Olafsson, G., 1989. Quantitative alcareous nannofossil biostratigraphy of upper Oligocene to middie Miocene sediment from ODP Hole 
667A and middle Miocene sediment from DSDP Site 574. In Ruddiman, W., Sarnthein, M., et al., Proc. ODP, Sci. Results, 108: College Station, TX (Ocean Drilling Program), 9-22.

Parker, M. E., Clark, M., and Wise, S. W., Jr., 1985. Calcareous nannofossils of Deep Sea Drilling Project Sites 558 and 563, North Atlantic Ocean: biostratigraphy and the distribution of Braarudosphaerids. In Bougault, H., Cande, S. C., et al., Init. Repts. DSDP, 82: Washington (U.S. Govt. Printing Office), 559-589.

Pelosio, G., Raffi, S., and Rio, D., 1980. The Plio-Pleistocene boundary controversy. Status in 1979 at the light of International Stratigraphic Guide. Volume dedicato a Sergio Venzo, Parma, 131-140.

Perch-Nielsen, K., 1977. Albian to Pleistocene calcareous nannofossils from the western South Atlantic Deep Sea Drilling Project, Leg 39. In Supko, P. R., Perch-Nielsen, K., et al., Init. Repts. DSDP, 39: Washington (U.S. Govt. Printing Office), 699-823. 1985. Cenozoic calcareous nannofossils. In Bolli, H. M. Saunders, J. B., and Perch-Nielsen, K. (Eds.), Plankton Stratigraphy: Cambridge (Cambridge Univ. Press), 427-553.

Prell, W. L., 1984. Covariance patterns of foraminiferal $\delta^{18} \mathrm{O}$ : an evolution of Pliocene ice volume changes near 3.2 million years ago. Science, 226:692-694.

Proto-Decima, F., Medizza, F., and Todesco, L., 1978. Southern Atlantic Leg 40, calcareous nannofossils. In Bolli, H. M., Ryan, W.B.F. et al., Init. Repts. DSDP, 40: Washington (U.S. Govt. Printing Office), 571-634.

Pujos, A., 1985. Cenozoic nannofossils, central equatorial Pacific, Deep Sea Drilling Project Leg 85. In Mayer, L., Theyer, F., Thomas, E., et al., Init. Repts. DSDP, 85: Washington (U.S. Govt. Printing Office), 581-608.

Raffi, I., and Rio, D., 1979. Calcareous nannofossil biostratigraphy of DSDP Site 132-Leg 13 (Tyrrhenian Sea-Western Mediterranean). Riv. It. Paleont., Stratigr., 85:127-172.

Ramsay, A.T.S., 1972. Aspect of the distribution of fossil species of calcareous nannoplankton in North Atlantic and Carribbean sediments. Nature, 236:67-70.

1977. Sedimentological clues to paleooceanography. In Ramsay, A.T.S. (Ed.), Oceanic Micropaleontology (Vol. 2): New York (Academic Press), 1371-1453.

Ramsay, A.T.S., Schneidermann, N., and Finch, J. W., 1973. Fluctations in the past rates of carbonate solution at Site 149: a comparison with other ocean basins and an interpretation of their significance. In Edgard, N. T., Saunders, J. B., et al., Init. Repts. DSDP, 15: Washington (U.S. Govt. Printing Office), 805-811.

Rio, D., 1982. The fossil distribution of coccolithophore genus Gephyrocapsa Kamptner and related Plio-Pleistocene chronostratigraphic problems. In Prell, W. L., Gardner, J. V., et al., Init. Repts. DSDP, 68: Washington (U.S. Govt. Printing Office), 325-343.

Rio, D., Backman, J., and Raffi, I., in press a. Calcareous nannofossil biochronology and the Pliocene-Pleistocene boundary. In Van Couvering, J. A. (Ed.), Final Repts., IGCP Project, No. 41.

Rio, D., Fornaciari, E., and Olafsson, G., in press b. New Miocene calcareous nannofossil species from tropical Indian Ocean. Mem. Sci. Geol.

Rio, D., Raffi, I., and Villa, G., 1989. Pliocene-Pleistocene calcareous nannofossil distribution patterns in the western Mediterranean. In Kastens, K., Mascle, J., et al., Proc. ODP, Sci. Results, 107: College Station, TX (Ocean Drilling Program), 513-533.

Rio, D., Sprovieri, R., Raffi, I., and Valleri, G., 1988. Biostratigrafia e paleoecologia della sezione stratotipica del Piacenziano. Bull. Soc. Paleontol. It., 27(2):213-238.

Roth, P. H., 1973. Calcareous nannofossils-Leg 17, Deep Sea Drilling Project. In Winterer, E. L., Ewing, J. I., et al., Init. Repts. DSDP, 17: Washington (U.S. Govt. Printing Office), 695-795.

1974. Calcareous nannofossils from the northwestern Indian Ocean Leg 24, Deep Sea Drilling Project. In Fisher, R. L., Bunce, E. T., et al., Init. Repts. DSDP, 24: Washington (U.S. Govt. Printing Office), 969-994.

1983. Jurassic and Lower Cretaceous calcareous nannofossils in the western North Atlantic (Site 534): biostratigraphy, preservation, and some observations on biogeography and paleoceanography. In Sheridan, R. E., Gradstein, F. M., et al., Init. Repts. DSDP, 76: Washington (U.S. Govt. Printing Office), 587-621.

Roth, P. H., and Thierstein, H., 1972. Calcareous nannoplankton, Leg 14 of the Deep Sea Drilling Project. In Hayes, D. E., Pimm, A. C., et al., Init. Repts. DSDP, 14: Washington (U.S. Govt. Printing Office), $421-485$.

Ruddiman, W., Sarnthein, M., et al., 1989. Proc. ODP, Sci. Results, 108: College Station, TX (Ocean Drilling Program).

Salis, A. K., von, 1984. Miocene calcareous nannofossil biostratigraphy of Deep Sea Drilling Project Hole 521A (southeast Atlantic). In Hsü, K. J., La Brecque, J. L., et al., Init. Repts. DSDP, 73: Washington (U.S. Govt. Printing Office), 425-427.

Schlich, R., 1982. The Indian Ocean: aseismic ridges, spreading centers, and oceanic ridges. In Nairn, A.E.M., and Stehli, F. G. (Eds.), The Ocean Basins and Margins, The Indian Ocean: New York (Plenum Press), 51-147.

Schneidermann, N., 1973. Deposition of coccoliths in the compensation zone of the Atlantic ocean. In Smiths, L. A., and Hardenbol, J. (Eds.), Proceedings of a Symposium on Calcareous Nannofossils: Houston, TX (Soc. Econ. Paleontol. Mineral.), 140-151.

1977. Selective dissolution of recent coccoliths in the Atlantic Ocean. In Ramsay, A.T.S. (Ed.), Oceanic Micropaleontology (Vol. 2): New York (Academic Press), 1009-1046.

Shackleton, N. J., Backman, J., Zimmerman, H. B., Kent, D. V., Hall, M. A., Roberts, D. G., Schnitker, D., Baldauf, J. G., Desprairies, A., Homrighausen, R., Huddleston, P., Keene, J. B., Kaltenback, A. J., Krumsiek, K. A., Morton, A. C., Murray, J. W., and Westberg-Smith, J., 1984. Oxygen isotope calibration of the onset of icerafting in DSDP Site 552A: history of glaciation in North Atlantic region. Nature, 307:620-623.

Srinivasan, M. S., and Kennett, J. P., 1983. The Oligocene-Miocene boundary in the South Pacific. Geol. Soc. Am. Bull., 94:798-812.

Stradner, H., and Allram, F., 1982. The nannofossil assemblages of Deep Sea Drilling Project Leg 66, Middle America Trench. In Watkins, J. S., Moore, J. C., et al., Init. Repts. DSDP, 66: Washington (U.S. Govt. Printing Office), 589-639.

Takayama, T., and Sato, T., 1985. Coccolith biostratigraphy of the North Atlantic Ocean, Deep Sea Drilling Project Leg 94. In Ruddiman, W. F., Kidd, R. B., Thomas, E., et al., Init. Repts. DSDP, 94, Pt. 2: Washington (U.S. Govt. Printing Office), 651-702.

Tauxe, L., Opdyke, N. D., Pasini, G., and Elmi, C., 1983a. The paleomagnetism of the Vrica section (Calabria, Italy), the proposed Pliocene-Pleistocene boundary-stratotype section. Nature, 304:125129.

Tauxe, L., Tucker, P., Petersen, N. P., and LaBrecque J. P., 1983b. The magnetostratigraphy of Leg 73 sediments. Palaeogeogr., Palaeoclimatol., Palaeoecol., 42:65-90.

Theodoridis, S., 1984. Calcareous nannofossil biozonation of the Miocene and revision of the helicoliths and discoasters. Utrecht Micropaleontol. Bull., 32:271.

Thierstein, H. R., 1974. Calcareous nannoplankton-Leg 26 Deep Sea Drilling Project. In Davies, T. A., Luyendyk, B. P., et al. Init. Repts. $D S D P, 26:$ Washington (U.S. Govt. Printing Office), 619-667.

Thierstein, H. R., Geitzenauer, K. R., Molfino, B., and Shackleton, N. J., 1977. Global synchroneity of late Quaternary coccolith datum levels: validation by oxygen isotopes. Geology, 5:400-404.

Thunell, R. C., 1976. Optimum indices of calcium carbonate dissolution in deep-sea sediments. Geology, 4:525-528.

Van Heck, S., 1979a. Bibliography and taxa of calcareous nannoplankton. INA Newsl., 1(1):AB1-B27.

1979b. Bibliography and taxa of calcareous nannoplankton. INA Newsl., 1(2):13-42.

1980a. Bibliography and taxa of calcareous nannoplankton. INA Newsl., 2(1):54-34.

1980b. Bibliography and taxa of calcareous nannoplankton. INA Newsl., 2(2):43-81.

1981a. Bibliography and taxa of calcareous nannoplankton. INA Newsl., 3(1):4-41.

1981b. Bibliography and taxa of calcareous nannoplankton.

INA Newsl., 3(2):51-86.

1982a. Bibliography and taxa of calcareous nannoplankton. INA Newsl., 4(1):7-50.

1982b. Bibliography and taxa of calcareous nannoplankton. INA Newsl., 4(2):65-96.

Williams, D. F., Thunell, R. C., Tappa, E., Rio, D., and Raffi, I., 1988. Chronology of the Pleistocene oxygen isotope record: $0-1.88$ million years before present. Palaeogeogr., Palaeoclimatol., Palaeoecol., 64:221-240. 
Wise, S. W., Jr., 1973. Calcareous nannofossils from cores recovered during Leg 18, Deep Sea Drilling Project: biostratigraphy and observations of diagenesis. In Kulm, L. D., von Huene, R., et al., Init. Repts. DSDP, 18: Washington (U.S. Govt. Printing Office), 569-615. 1976. Chalk formation: early diagenesis. In Andersen, N. A., and Malahoff, A. (Eds.), The Fate of Fossil Fuel $\mathrm{CO}_{2}$ in the Oceans: New York (Plenum Press), 717-739.

Woodruff, F., Savin, S. M., and Douglas, R. G., 1981. Miocene stable isotope record: a detailed deep Pacific Ocean study, and its paleoclimatic implications. Science, 212:665-668.

Zijderveld, J.D.A., Zachariasse, J. W., Verhallen, P.J.J., and Hilgen, F. J., 1986. The age of the Miocene/Pliocene boundary. Newsl. Stratigr., 16:169-181.

Date of initial receipt: 6 June 1989

Date of acceptance: 15 January 1990

Ms 115B-152

\section{APPENDIX}

Calcareous nannofossils considered in this chapter (in alphabetic order of generic epithets)

Amaurolithus amplificus (Bukry and Percival, 1971) Gartner and Bukry, 1975

Amaurolithus delicatus Gartner and Bukry, 1975

Amaurolithus primus (Bukry and Percival, 1971) Gartner and Bukry, 1975

Amaurolithus tricorniculatus (Gartner, 1967) Gartner and Bukry, 1975

Calcidiscus leptoporus (Murray and Blackman, 1898) Loeblich and Tappan, 1978

Calcidiscus macintyrei (Bukry and Bramlette, 1969) Loeblich and Tappan, 1978

Calcidiscus premacintyrei Theodoridis, 1984

Catinaster calyculus Martini and Bramlette, 1963

Catinaster coalitus Martini and Bramlette, 1965

Ceratolithus acutus Gartner and Bukry, 1974

Ceratolithus armatus Müller, 1974

Ceratolithus cristatus Kamptner, 1950

Ceratolithus rugosus Bukry and Bramlette, 1968

Ceratolithus telesmus Norris, 1965

Clausicoccus fenestratus (Deflandre and Fert, 1954) Prins, 1979

Coccolithus eopelagicus (Bramlette and Riedel, 1954) Bramlette and Sullivan, 1961

Coccolithus miopelagicus Bukry, 1971

Coccolithus pelagicus (Wallich, 1877) Schiller, 1930

Coccolithus radiatus Kamptner, 1955

Coronocyclus nitescens (Kamptner, 1963) Bramlette and Wilcoxon, 1967

Cyclicargolithus abisectus (Müller, 1970) Wise, 1973

Cyclicargolithus floridanus (Roth and Hay in Hay et al., 1967) Bukry, 1971

Dictyococcites antarcticus Haq, 1967 = Dictyococcites perplexus

Dictyococcites bisectus (Hay, Mohler, and Wade, 1966) Bukry and Percival, 1971

Dictyococcites hesslandii (Haq, 1966) Haq and Lohman, 1976

Dictyococcites productus (Kamptner, 1963) Backman, 1980

Discoaster adamanteus Bramlette and Wilcoxon, 1967

Discoaster asymmetricus Gartner, 1969

Discoaster aulakos Gartner, 1967

Discoaster bellus Bukry and Percival, 1971

Discoaster berggrenii Bukry, 1971

Discoaster blackstockae Bukry, 1973

Discoaster bollii Martini and Bramlette, 1963

Discoaster braarudii Bukry, 1971

Discoaster brouweri Tan (1927) emend. Bramlette and Riedel, 1954

Discoaster calcaris Gartner, 1967

Discoaster calculosus Bukry, 1971

Discoaster challengeri Bramlette and Riedel, 1954

Discoaster decorus (Bukry, 1971) Bukry, 1973

Discoaster deflandrei Bramlette and Riedel, 1954

Discoaster dilatus Hay, 1967

Discoaster druggii Bramlette and Wilcoxon, 1967

Discoaster exilis Martini and Bramlette, 1963

Discoaster extensus Hay, 1967
Discoaster formosus Martini and Worsley, 1971

Discoaster hamatus Martini and Bramlette, 1963

Discoaster icarus Stradner, 1973

Discoaster intercalaris Bukry, 1971

Discoaster kugleri Martini and Bramlette, 1963

Discoaster loeblichii Bukry, 1971

Discoaster misconceptus Theodoridis, 1984 = Discoaster pentaradiatus

Discoaster moorei Bukry, 1971

Discoaster musicus Stradner, 1959

Discoaster neohamatus Bukry and Bramlette, 1969

Discoaster neorectus Bukry, 1971

Discoaster pansus (Bukry and Percival, 1971) Bukry, 1973

Discoaster pentaradiatus Tan (1927) emend. Bramlette and Riedel, 1954

Discoaster perclarus Haq in Haq et al., 1967

Discoaster phyllodus Hay, 1967

Discoaster prepentaradiatus Bukry and Percival, 1971

Discoaster pseudovariabilis Martini and Worsley, 1971

Discoaster quinqueramus Gartner, 1969

Discoaster sanmiguelensis Bukry, $1981=$ Discoaster musicus

Discoaster signus Bukry, 1971

Discoaster subsurculus Gartner, 1967

Discoaster surculus Martini and Bramlette, 1963

Discoaster tamalis Kamptner, 1967

Discoaster triradiatus Tan, 1927

Discoaster tristellifer Bukry, 1976

Discoaster tuberi Filewicz, 1984

Discoaster variabilis Martini and Bramlette, 1963

Discoaster woodringii Bramlette and Riedel, 1954

Ericsonia fenestrata (Deflandre and Fert, 1954) Stradner in Stadner and Edwards, 1968 = Clausicoccus fenestratus

Ericsonia obruta Perch-Nielsen, 1971

Geminilithella rotula (Kamptner, 1956) Backman, 1980

Gephyrocapsa caribbeanica Boudreaux and Hay, 1969

Gephyrocapsa oceanica Kamptner, 1943

Gephyrocapsa parallela Hay and Beaudry, 1973

Hayella aperta Theodoridis, 1984

Helicosphaera ampliaperta Bramlette and Wilcoxon, 1967

Helicosphaera carteri (Wallich, 1877) Kamptner, 1954

Helicosphaera compacta Bramlette and Wilcoxon, 1967

Helicosphaera crouchii Bukry, 1981 = Helicosphaera mediterranea

Helicosphaera euphratis Haq, 1966

Helicosphaera intermedia Martini, 1965

Helicosphaera mediterranea Müller, 1981

Helicosphaera obliqua Bramlette and Wilcoxon, 1967

Helicosphaera perch-nielsinae $\mathrm{Haq}, 1971$

Helicosphaera recta Haq, 1966

Helicosphaera sellii Bukry and Bramlette, 1969

Ilselithina fusa Roth, 1970

Minylitha convallis Bukry, 1973

Orthorhabdus serratus Bramlette and Wilcoxon, 1967 = Triquetrorabdulus serratus

Pseudoemiliania lacunosa (Kamptner, 1963) Gartner, 1969

Reticulofenestra daviesii (Haq, 1968) Backman, 1980

Reticulofenestra gelida (Geitzehanauer, 1972) Backman, 1978

Reticulofenestra haqii Backman, 1978

Reticulofenestra lockeri Müller, 1970

Reticulofenestra minuta Roth, 1970

Reticulofenestra minutula (Gartner, 1967) Haq and Berggren, 1978

Reticulofenestra pseudoumbilicus (Gartner, 1967) Gartner, 1969

Solidopons petrae Theodoridis, 1984

Sphenolithus abies Deflandre in Deflandre and Fert, 1954

Sphenolithus belemnos Bramlette and Wilcoxon, 1967

Sphenolithus calyculus Bukry, 1985

Sphenolithus capricornutus Bukry and Percival, 1971

Sphenolithus ciperoensis Bramlette and Wilcoxon, 1967

Sphenolithus compactus Backman, 1980

Sphenolithus conicus Bukry, 1971

Sphenolithus delphix Bukry, 1973

Sphenolithus dissimilis Bukry and Percival, 1971

Sphenolithus distentus (Martini, 1965) Bramlette and Wilcoxon, 1967

Sphenolithus heteromorphus Deflandre, 1953

Sphenolthus milanettii Olafsson and Rio in Rio et al., in press b

Sphenolithus moriformis (Brönnimann and Stradner, 1960) Bramlette and Wilcoxon, 1967 
Sphenolithus multispinatus Fornaciari and Rio in Rio et al., in press b Sphenolithus neoabies Bukry and Bramlette, 1969

Sphenolithus pacificus Martini, 1965

Sphenolithus pseudoheteromorphus Fornaciari and Rio in Rio et al., in press b

Sphenolithus predistentus Bramlette and Wilcoxon, 1967

Sphenolithus umbrellus (Bukry, 1971) Aubry and Knttel, 1986

Triquetrorhabdulus auritus Stradner and Allram, 1982

Triquetrorhabdulus backmanii Fornaciari and Rio in Rio et al., in press b Triquetrorhabdulus carinatus Martini, 1965

Triquetrorhabdulus curvus Fornaciari and Rio in Rio et al., in press b Triquetrorhabdulus challengeri Perch-Nielsen, 1977
Triquetrorhabdulus extensus Theodoridis, 1984

Triquetrorhabdulus farnsworthii (Gartner, 1967) Perch-Nielsen, 1985

Triquetrorhabdulus milowii Bukry, 1971

Triquetrorhabdulus protodecimae Fornaciari and Rio in Rio et al., in press b

Triquetrorhabdulus rioensis Olafsson, 1989

Triquetrorhabdulus rugosus Bramlette and Wilcoxon, 1967

Triquetrorhabdulus serratus (Bramlette and Wilcoxon, 1967) Olafsson, 1989

Triquetrorhabdulus striatus Müller, 1974

Zygrhablithus bijugatus (Deflandre in Deflandre and Fert, 1954) Deflandre, 1959 


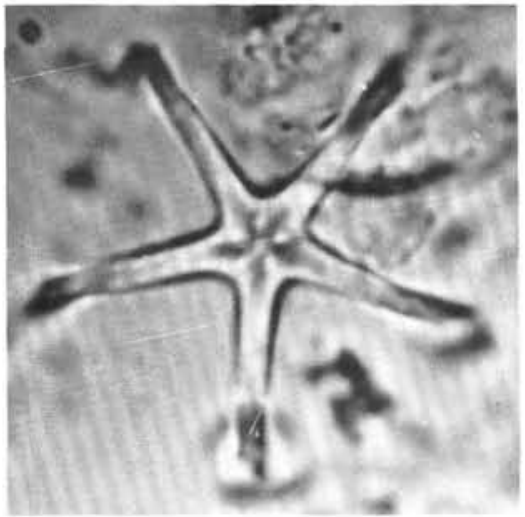

$1 \mathrm{~A}$

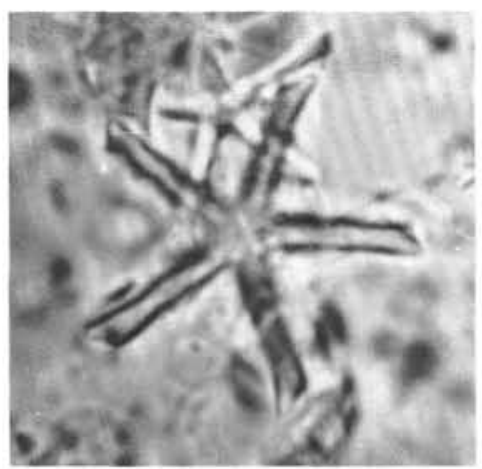

3

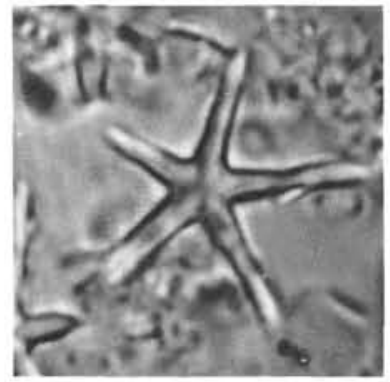

6

7

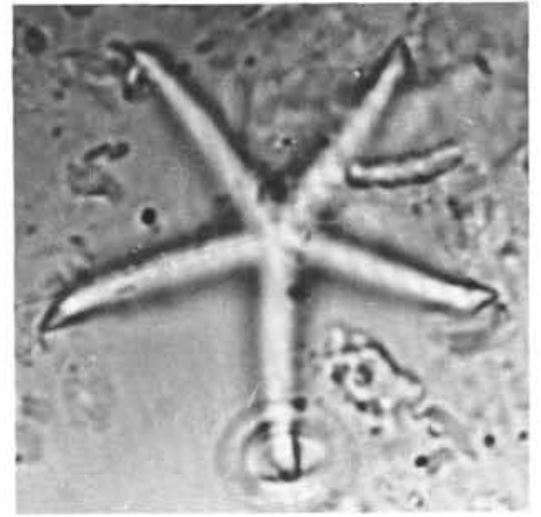

$1 B$

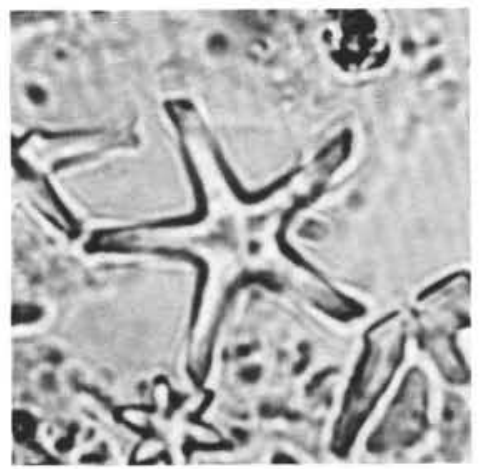

4

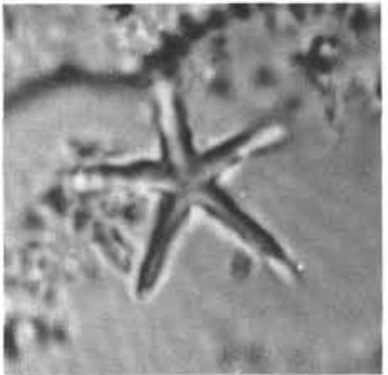

8A

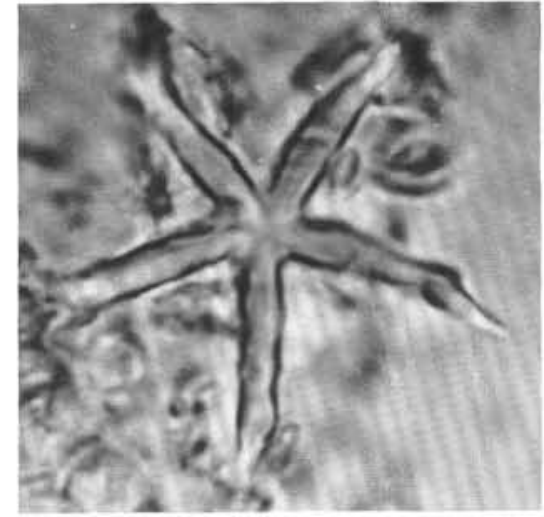

2

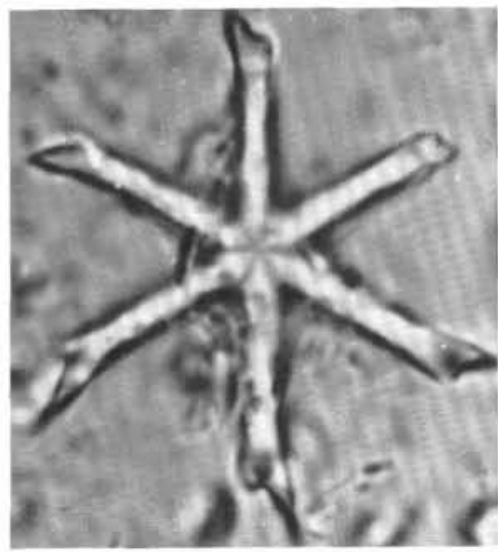

5

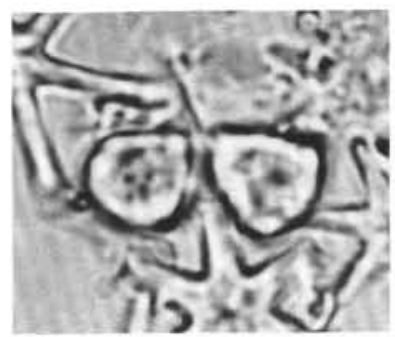

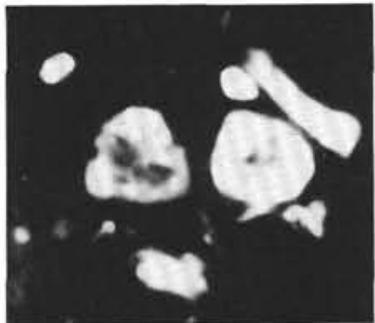

8B

Plate 1. All specimens $\times 2400$. 1-3. Discoaster hamatus Martini and Bramlette. (1a) Sample 115-710A-9H-5, $30 \mathrm{~cm}$; (1b) different focus of previous sample; (2) and (3) Sample 115-714A-7H-2, $10 \mathrm{~cm}$. 4. Discoaster hamatus-Discoaster bellus intergrade. Sample 115-710A-9H-5, $90 \mathrm{~cm}$. 5. Discoaster hamatus Martini and Bramlette, six-rayed form. Sample 115-714A-5H-3, $10 \mathrm{~cm}$. 6, 7. Discoaster bellus Bukry and Percival. Sample 115-714A-7H-2, $10 \mathrm{~cm}$. 8. Minylitha convallis Bukry. Sample 115-710A-8H-5, $30 \mathrm{~cm}$. (8a) Parallel light; (8b) crossed nicols. 


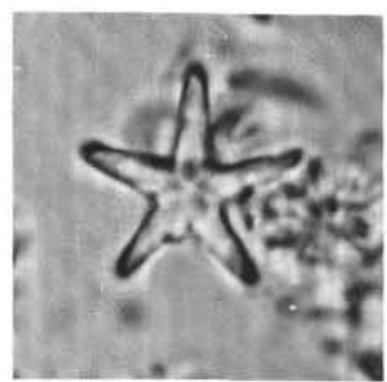

1

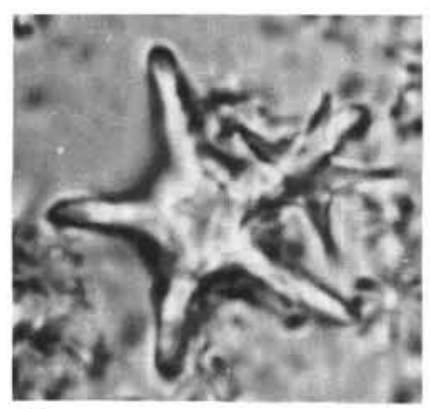

5

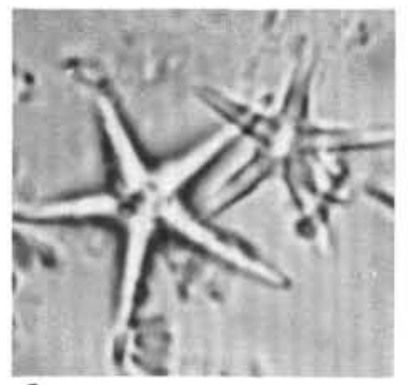

9

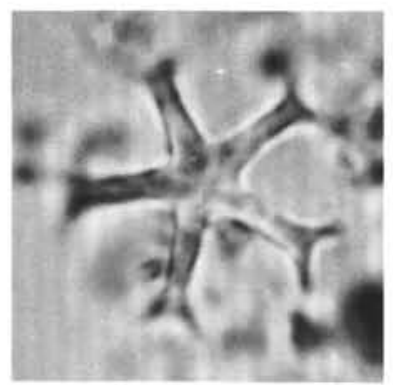

12

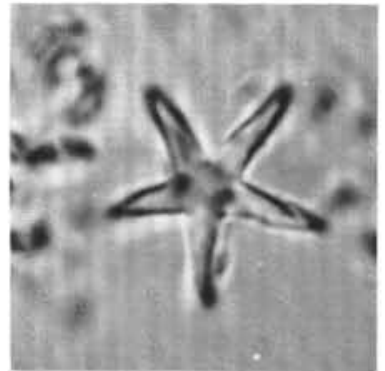

2

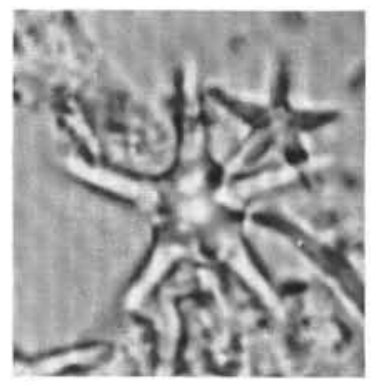

6

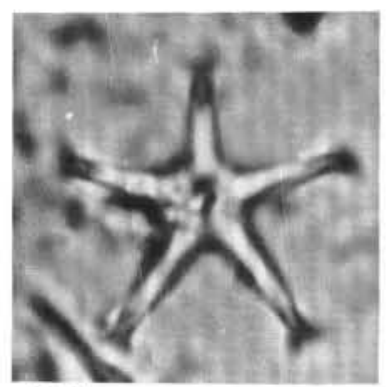

$10 \mathrm{~A}$

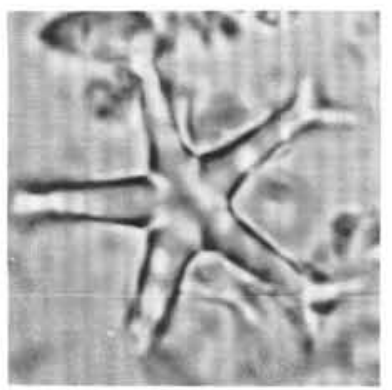

13

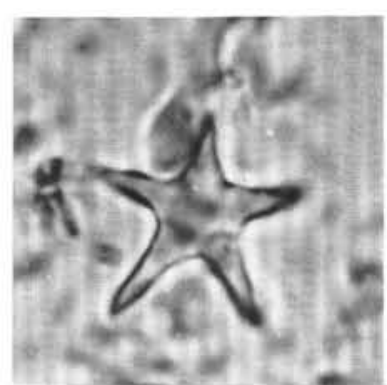

3

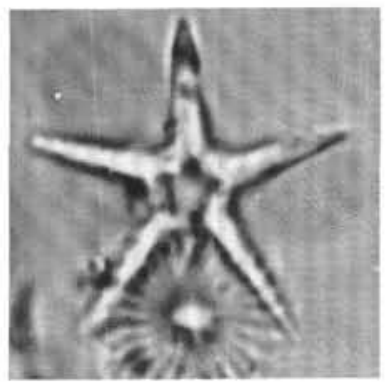

7

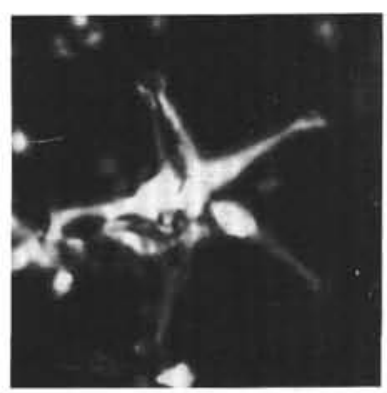

$10 \mathrm{~B}$

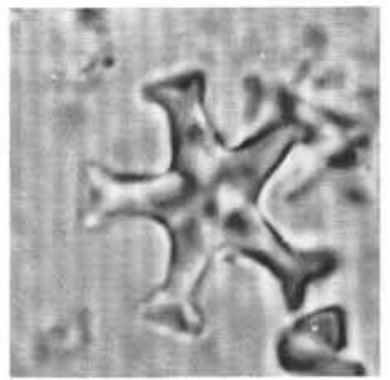

14

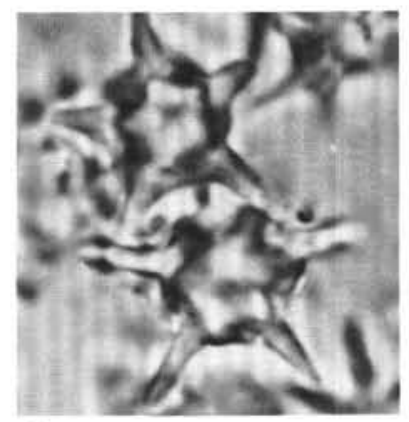

4

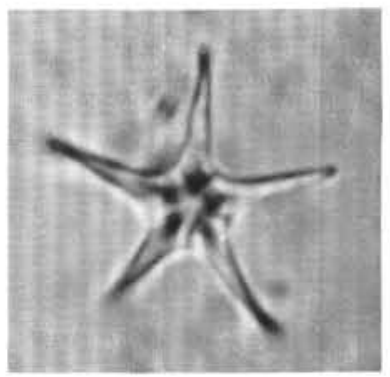

8

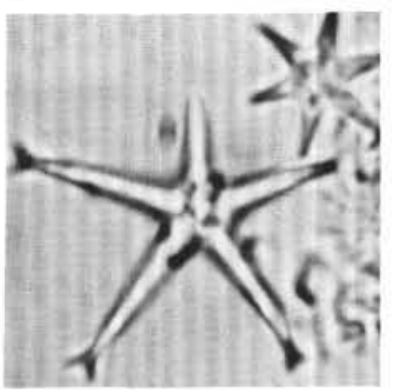

11

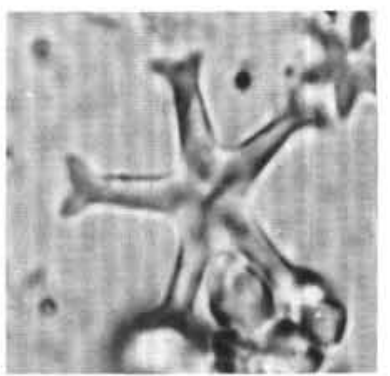

15

Plate 2. All specimens $\times 2400$. 1, 2. Discoaster bellus-Discoaster berggrenii intergrade. Sample 115-710A-7H-7, 30 cm. 3. Discoaster berggrenii Bukry, primitive form. Sample $115-710 \mathrm{~A}-7 \mathrm{H}-7,30 \mathrm{~cm}$. 4. Discoaster berggrenii Bukry, evoluted form. Sample $115-710 \mathrm{~A}-7 \mathrm{H}-3,130 \mathrm{~cm}$. 5. Discoaster berggrenii Bukry. Sample 115-710A-7H-5, $30 \mathrm{~cm}$. 6. Discoaster berggrenii-Discoaster quinqueramus intergrade. Sample 115-710A-7H-2, $30 \mathrm{~cm}$. 7, 8. Discoaster quinqueramus Gartner. (7) Sample 115-710B-6H-4, $30 \mathrm{~cm}$; (8) Sample 115-711B-4H-5, 50 cm. 9. Discoaster quinqueramus Gartner (lower left) and Discoaster sp. 1. (upper right). Sample 115-710B-6H-6, $30 \mathrm{~cm}$. 10, 11. Discoaster pentaradiatus (Tan Sin Hok). (10) Sample 115-710A-3H-3, $130 \mathrm{~cm}$; (10a) parallel light; (10b) crossed nicols; (11) Sample 115-710A-8H-5, $30 \mathrm{~cm}$. 12, 13. Discoaster prepentaradiatus Bukry and Percival. (12) Sample 115-710A-9H-1, $30 \mathrm{~cm}$; (13) Sample 115-714A-5H-3, $10 \mathrm{~cm}$. 14, 15. Discoaster moorei Bukry. Sample 115-710A-9H-6, $30 \mathrm{~cm}$. 


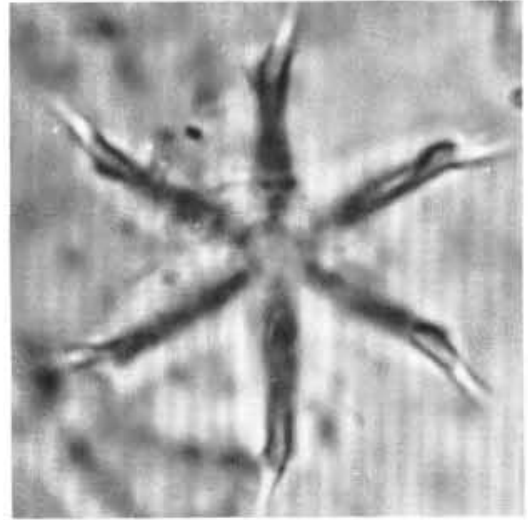

$1 \mathrm{~A}$

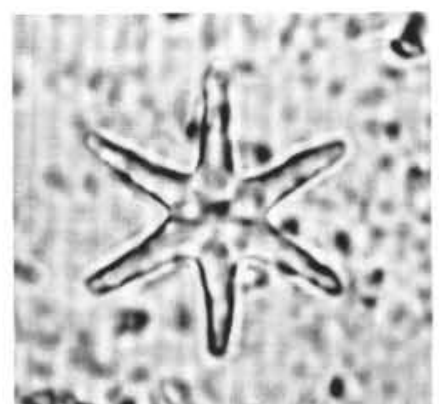

3

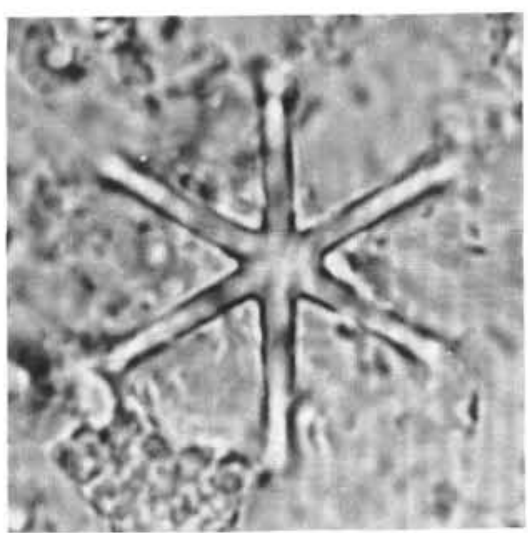

$1 B$

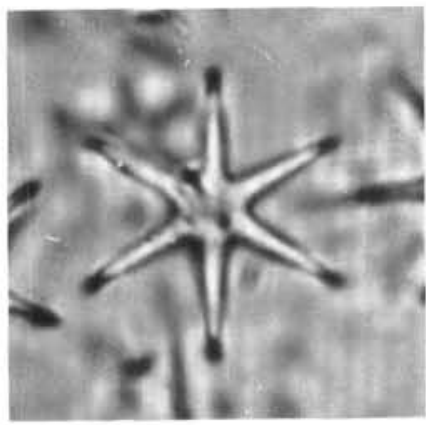

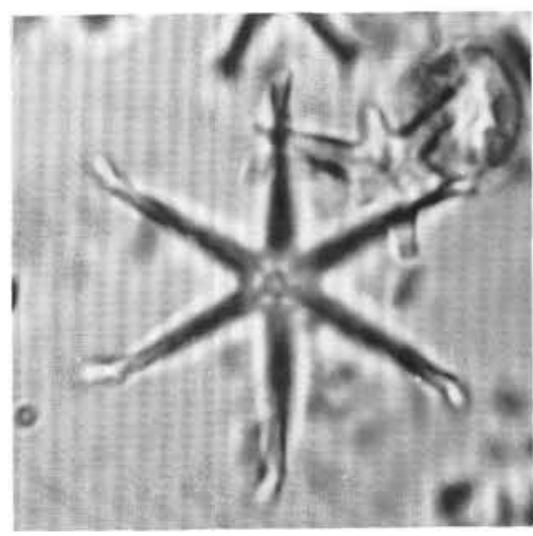

2

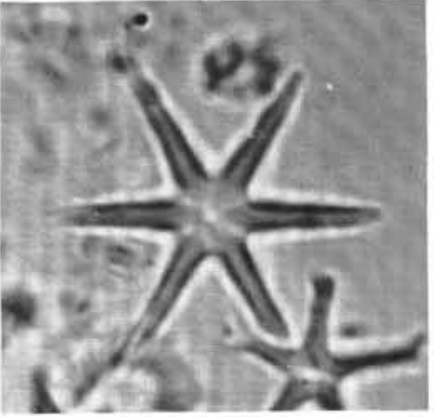

5

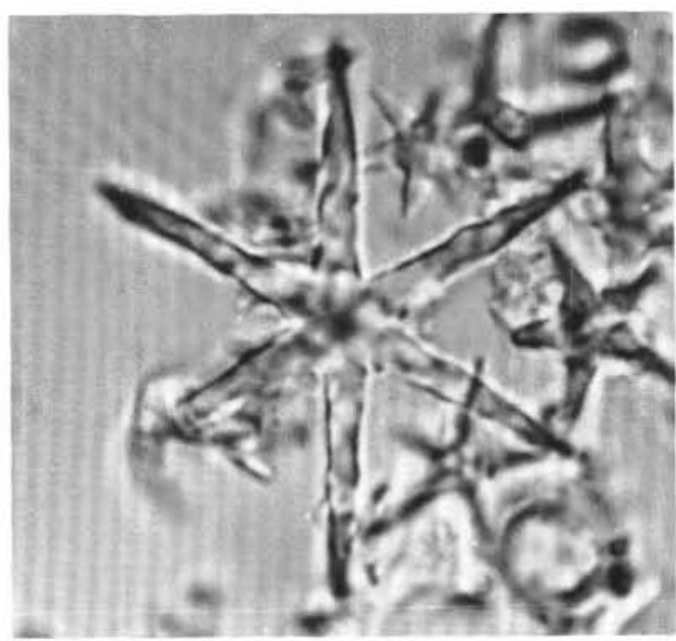

6
4

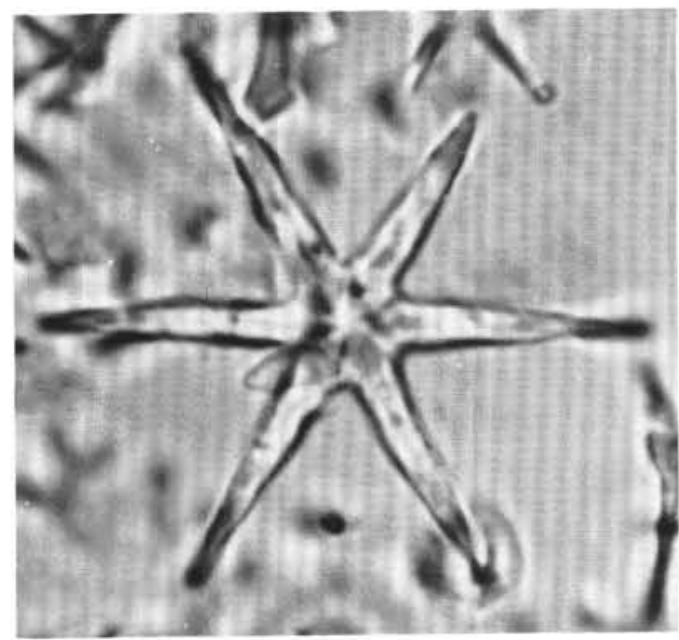

7

Plate 3. All specimens $\times 2400$. 1, 2. Discoaster neohamatus Bukry and Bramlette. (1a) Sample 115-714A-5H-3, $10 \mathrm{~cm}$; (1b) different focus of previous sample; (2) Sample 115-710A-9H-2, $90 \mathrm{~cm}$. 3-5. Discoaster brouweri (Tan Sin Hok). (3) Sample 115-714B-9H-4, 40 cm; (4) Sample 115-710A$3 \mathrm{H}-3,130 \mathrm{~cm}$; (5) Sample 115-710A-9H-2, $90 \mathrm{~cm}$. 6, 7. Discoaster sp. 2. (6) Sample 115-710A-7H-2, $30 \mathrm{~cm}$; (7) Sample 115-710B-6H-6, 30 cm. 


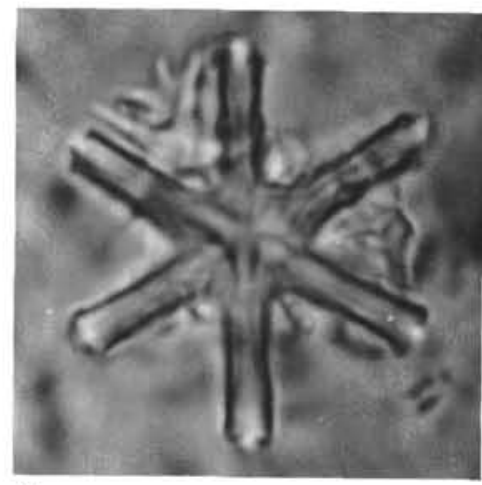

1

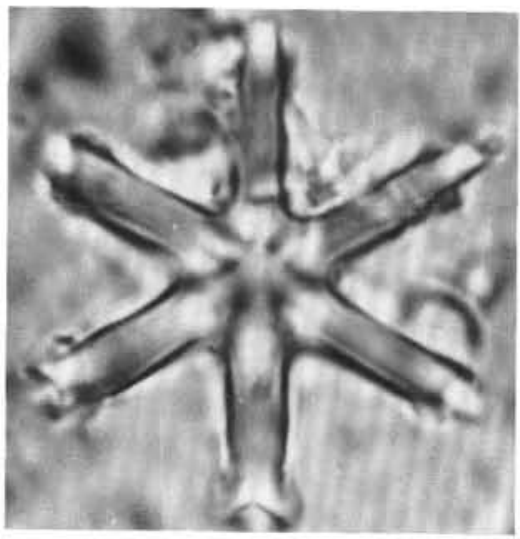

3

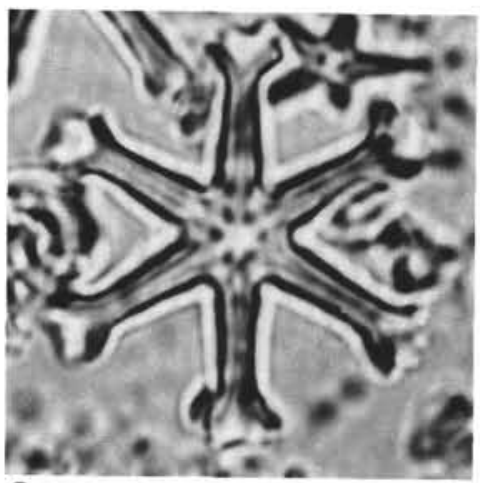

6

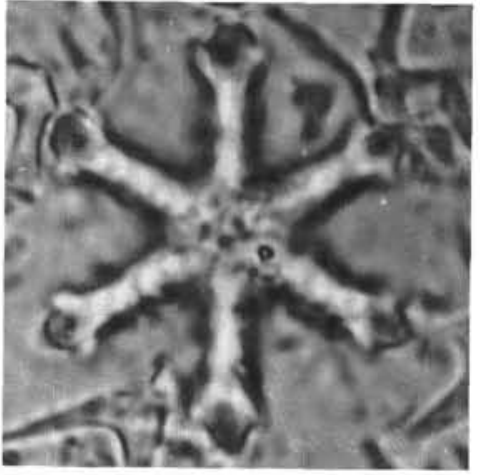

2A

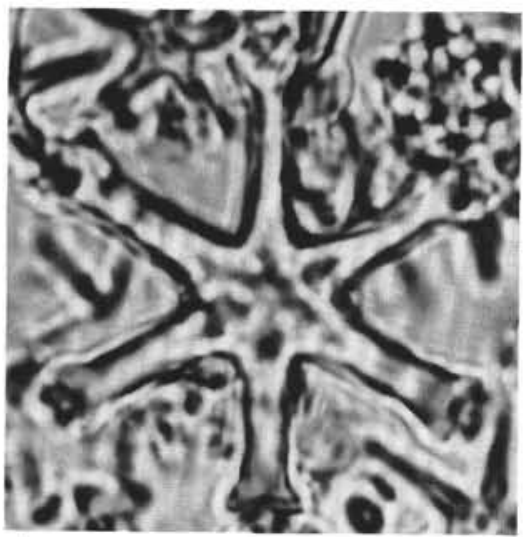

4

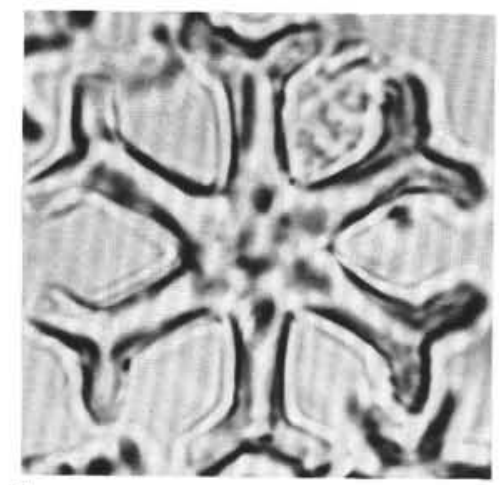

7

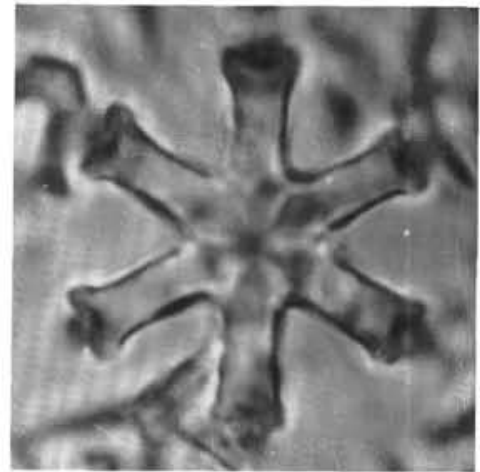

2B

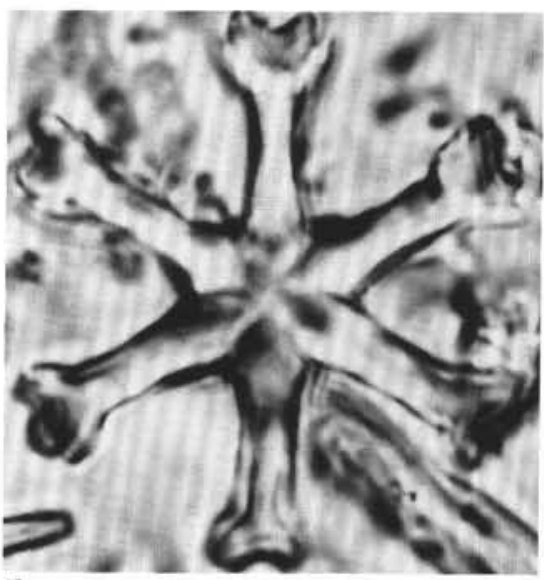

5

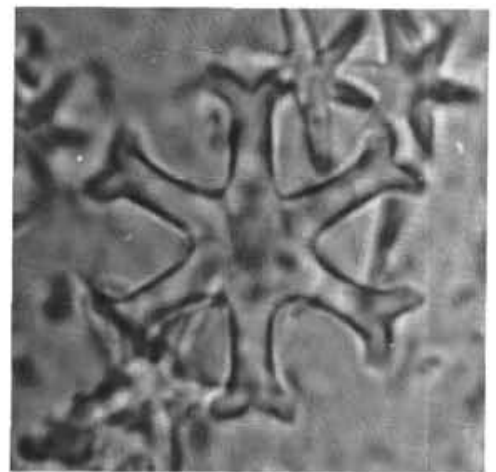

8

Plate 4. All specimens $\times 2400$. 1-4. Discoaster surculus Martini and Bramlette. (1) and (2a) Sample 115-710B-5H-6, 150 cm; (2b) different focus of previous sample; (3) Sample 115-711B-4H-6, $50 \mathrm{~cm}$; (4) Sample 115-710A-9H-5, $90 \mathrm{~cm}$. 5, 6. Discoaster pseudovariabilis Martini and Worsley. (5) Sample 115-714B-6H-7, $40 \mathrm{~cm}$; (6) Sample 115-710A-9H-6, $76 \mathrm{~cm}$. 7. Discoaster pansus (Bukry and Percival). Sample 115-710A-9H-3, 130 cm. 8. Discoaster variabilis Martini and Bramlette. Sample 115-710B-5H-6, $150 \mathrm{~cm}$. 


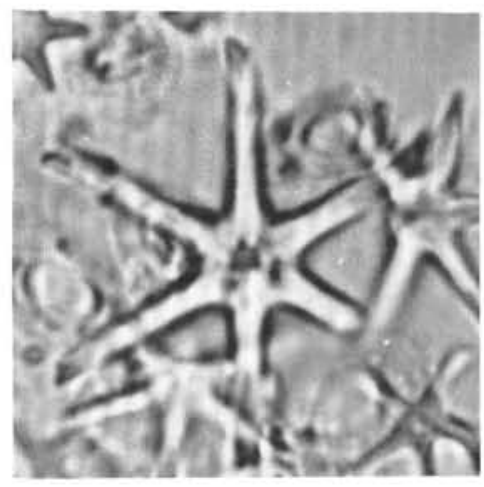

1

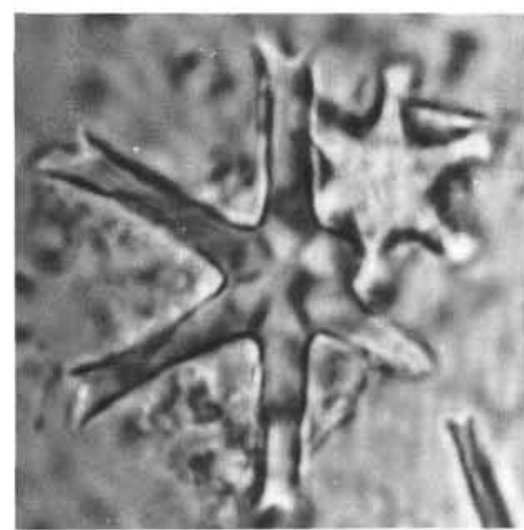

4

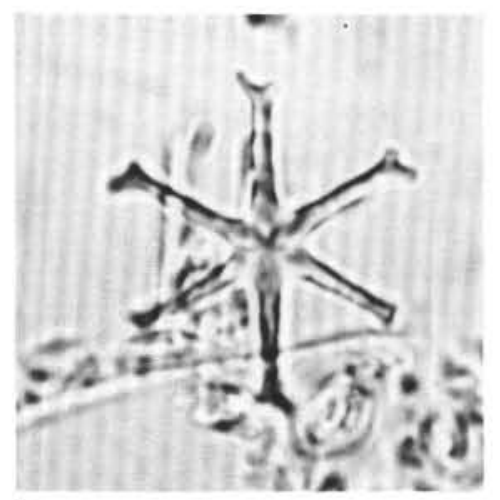

7

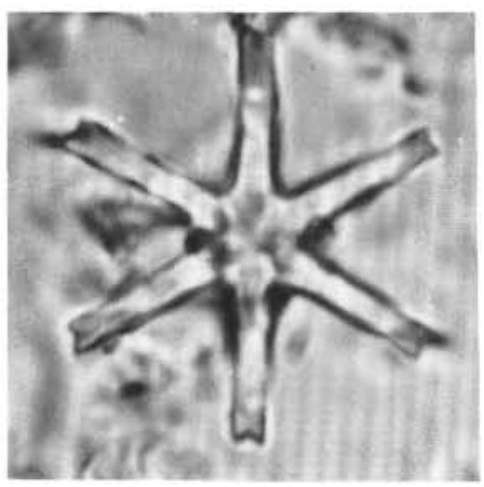

2

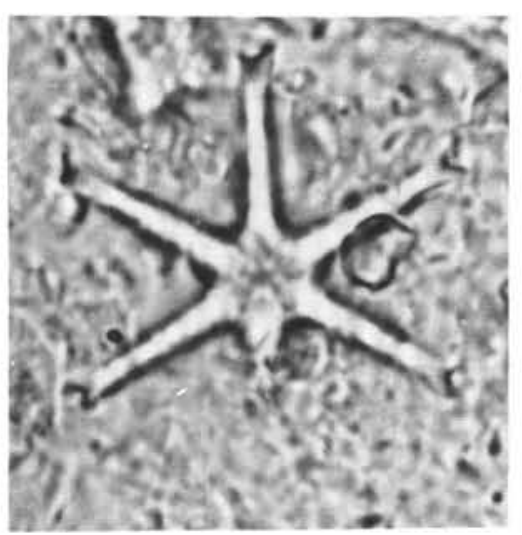

5

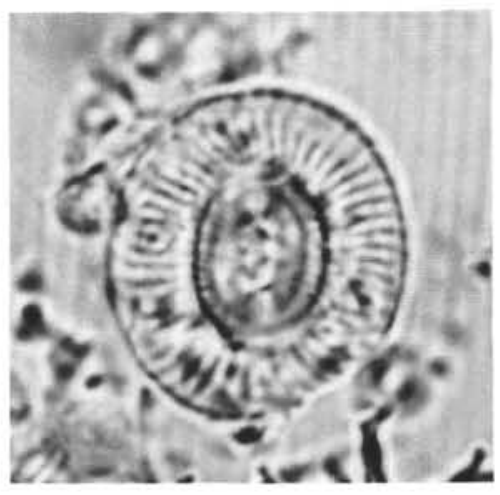

8A

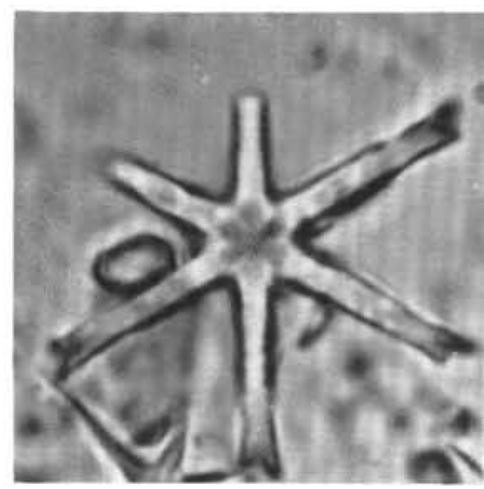

3

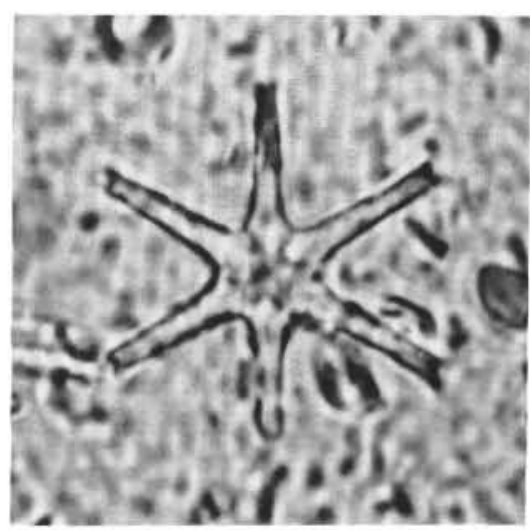

6

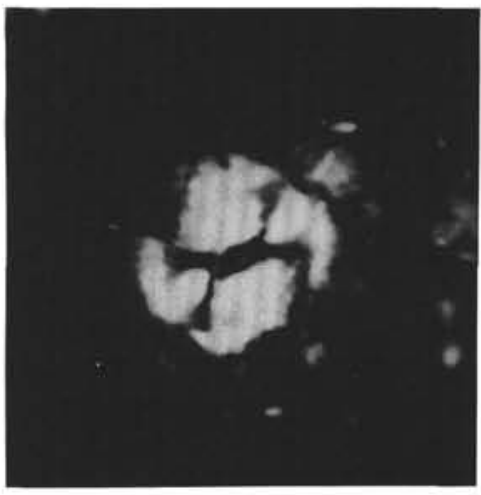

8B

Plate 5. All specimens $\times 2400$. 1-3. Discoaster calcaris Gartner. (1) Sample $115-710 \mathrm{~A}-9 \mathrm{H}-1,90 \mathrm{~cm}$; (2) and (3) Sample $115-714 \mathrm{~A}-5 \mathrm{H}-3,10 \mathrm{~cm}$. 4-6. Discoaster aff. calcaris. Sample 115-714B-9H-4, $40 \mathrm{~cm}$. 7. Discoaster exilis Martini and Bramlette. Sample 115-714A-8H-7, 30 cm. 8. Coccolithus miopelagicus Bukry. Sample 115-714A-8H-7, $30 \mathrm{~cm}$. (8a) Parallel light; (8b) crossed nicols. 


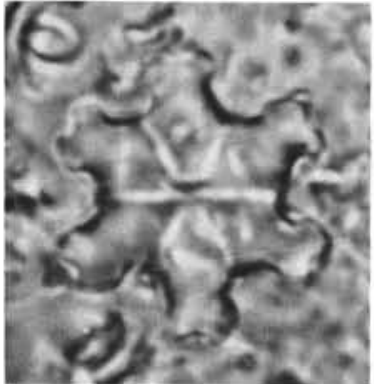

$1 \mathrm{~A}$

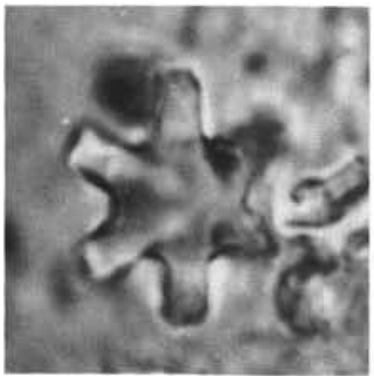

4

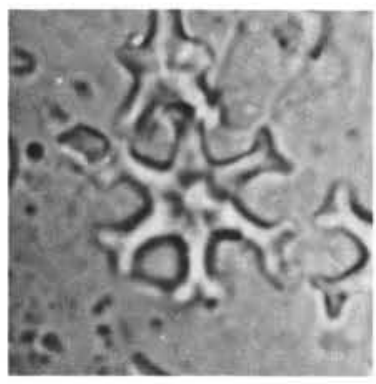

8

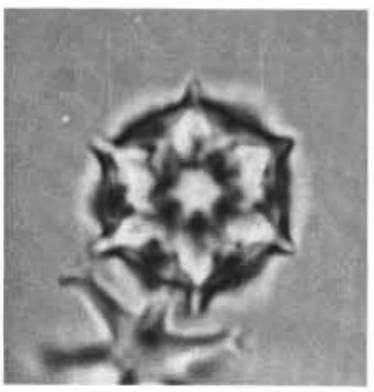

$11 \mathrm{~A}$

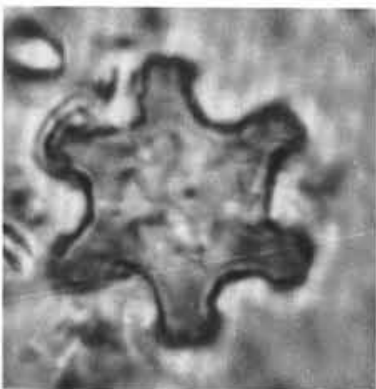

$1 B$

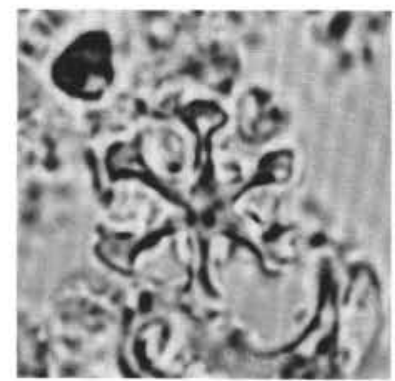

5

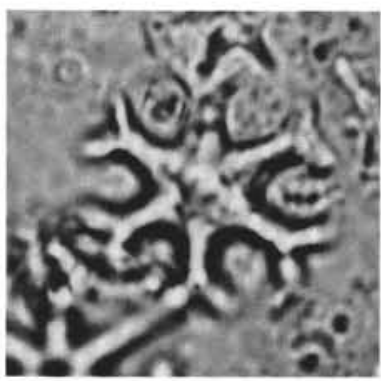

$9 \mathrm{~A}$

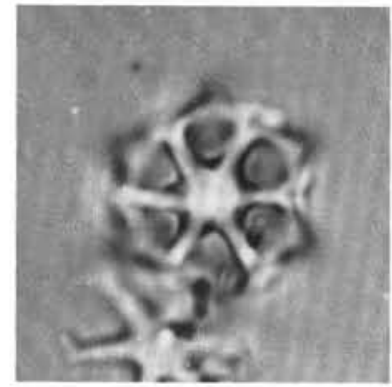

11B

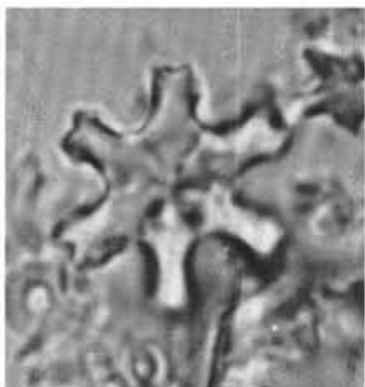

2

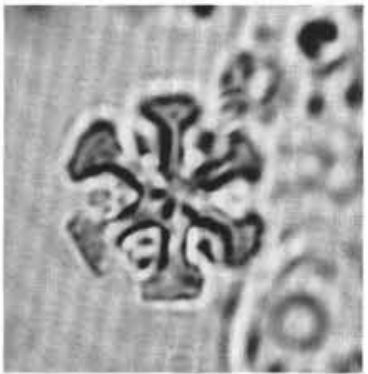

6

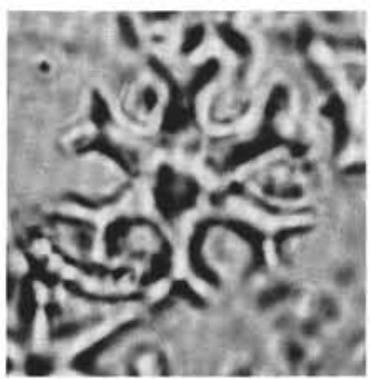

9B

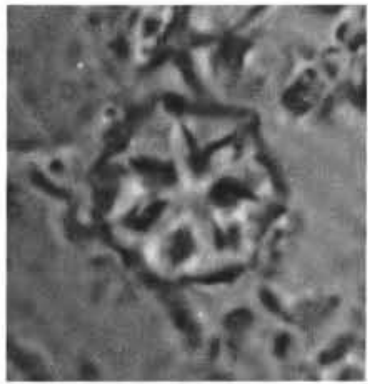

12

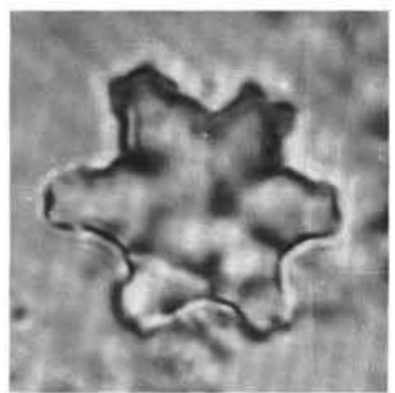

3

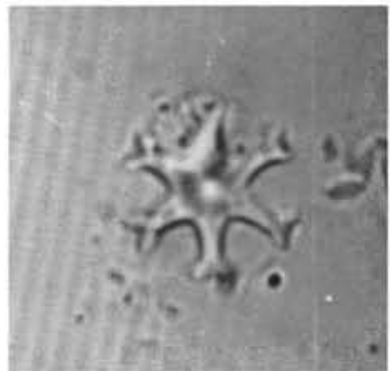

7

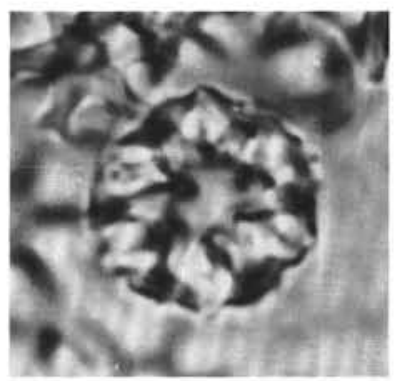

10

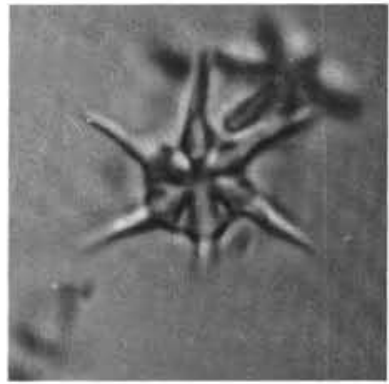

13

Plate 6. All specimens $\times 2400$. 1-3. Discoaster kugleri Martini and Bramlette. Sample $115-714 \mathrm{~B}-8 \mathrm{H}-4,40 \mathrm{~cm}$. 4. Discoaster cf. musicus. Sample 115-714B-9H-4, 40 cm. 5, 6. Discoaster subsurculus Gartner. Sample 115-714A-8H-4, 75 cm. 7-9. Discoaster bollii Martini and Bramlette. (7) and (8) Sample 115-710A-9H-5, $30 \mathrm{~cm}$; (9a) Sample 115-710A-9H-3, $90 \mathrm{~cm}$; (9b) different focus of previous sample. 10-12. Catinaster coalitus Martini and Bramlette. (10) and (12) Sample 115-710A-9H-5, $30 \mathrm{~cm}$; (11a) Sample 115-710A-9H-3, $130 \mathrm{~cm}$; (11b) different focus of previous sample. 13. Catinaster calyculus Martini and Bramlette. Sample $115-714 \mathrm{~A}-7 \mathrm{H}-2,10 \mathrm{~cm}$. 


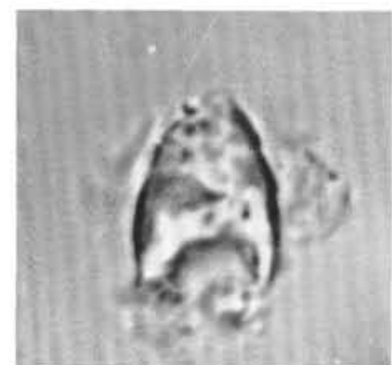

1

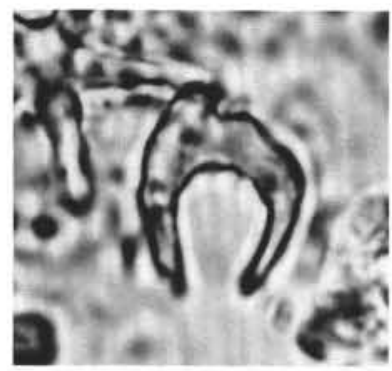

5

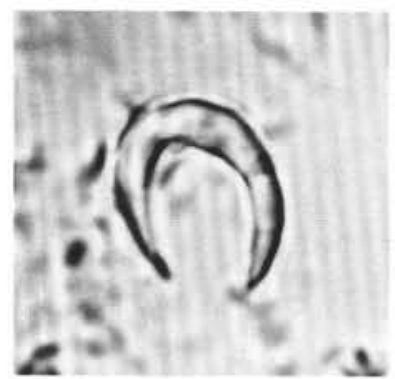

9

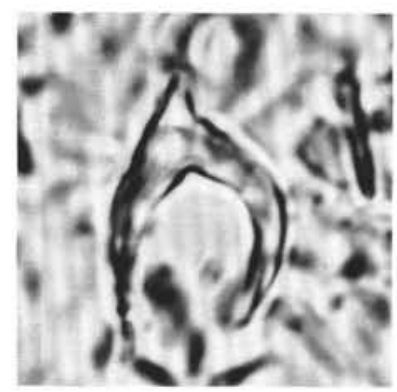

13

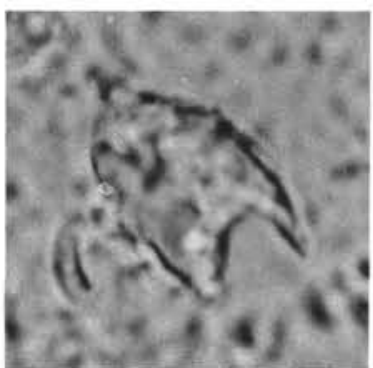

2

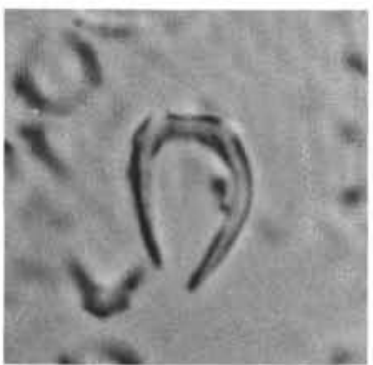

6

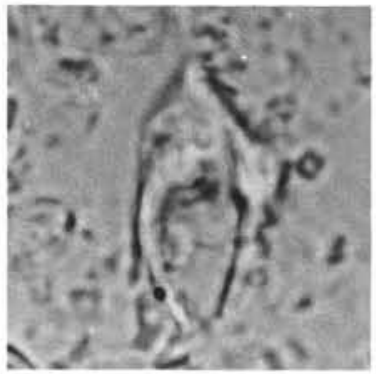

10

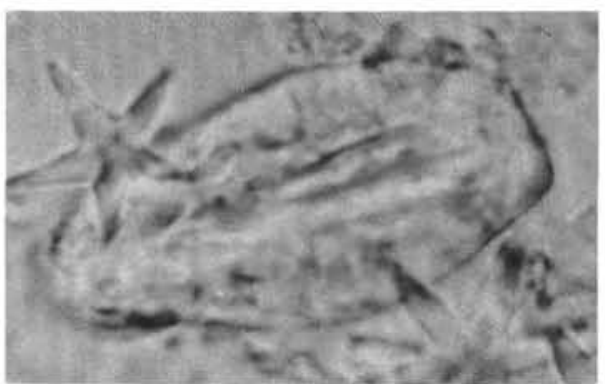

14

3

7

11
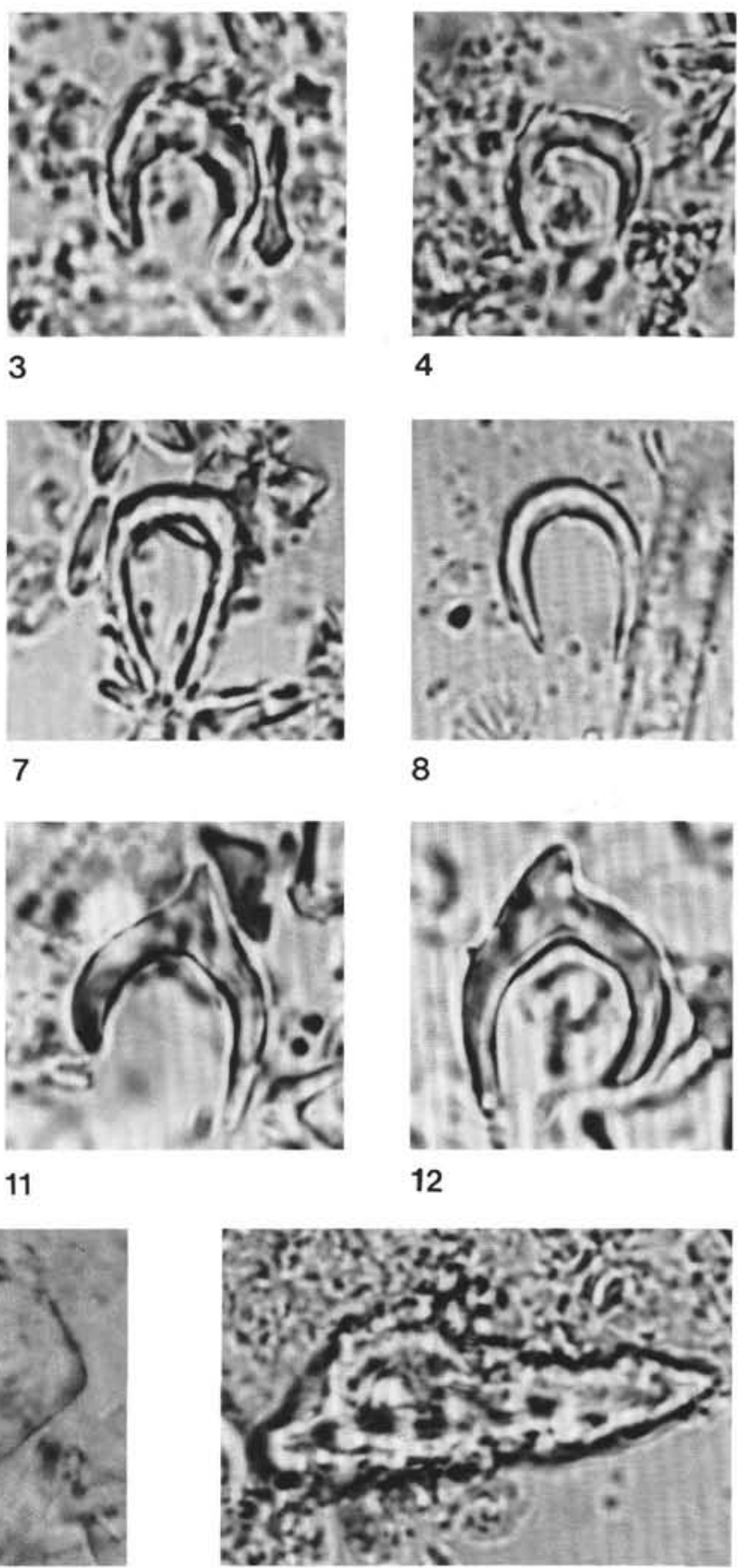

15

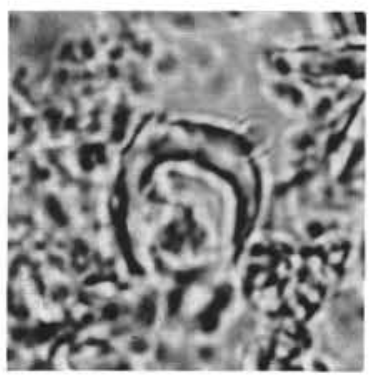

4

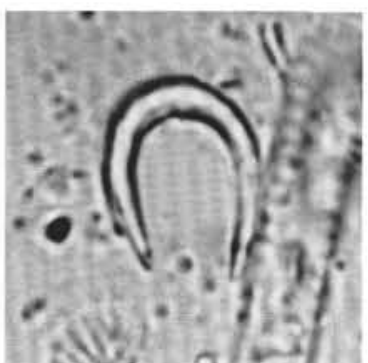

8

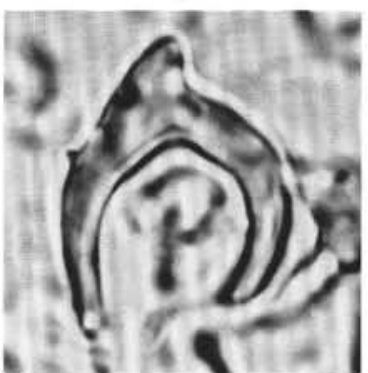

12

Plate 7. All specimens $\times 2400$. 1-4. Amaurolithus primus (Bukry and Percival). (1) Sample 115-710A-7H-2, $30 \mathrm{~cm}$; (2) Sample 115-709C-11H-4, $60 \mathrm{~cm}$; (3) Sample 115-713A-4H-6, $130 \mathrm{~cm}$; (4) Sample 115-713A-4R-4, $130 \mathrm{~cm}$. 5, 9. Amaurolithus primus-Amaurolithus delicatus intergrade. (5) Sample 115-713A-4R-6, $130 \mathrm{~cm}$; (9) Sample 115-710B-5H-2, $130 \mathrm{~cm}$. 6-8. Amaurolithus delicatus Gartner and Bukry. (6) Sample 115-710B-5H-6, $130 \mathrm{~cm}$; (7) Sample 115-713A-4R-4, $130 \mathrm{~cm}$; (8) Sample 115-710B-5H-4, $90 \mathrm{~cm}$. 10. Amaurolithus sp. Sample 115-709C-7H-4, 60 cm. 11-13. Amaurolithus tricorniculatus (Gartner). (11) Sample 115-710B-5H-1, $120 \mathrm{~cm} ;(12)$ and (13) Sample 115-710B-5H-1, 130 cm. 14, 15. Triquetrorhabdulus rugosus Bramlette and Wilcoxon. (14) Sample 115-709C-11H-6, $60 \mathrm{~cm}$; (15) Sample 115-713A-4R-4, $130 \mathrm{~cm}$. 


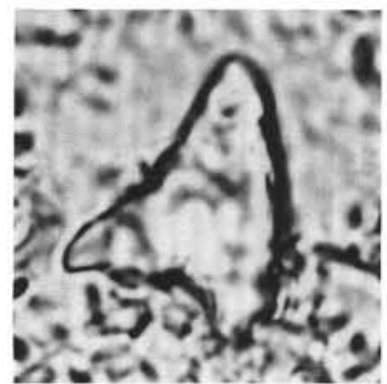

1

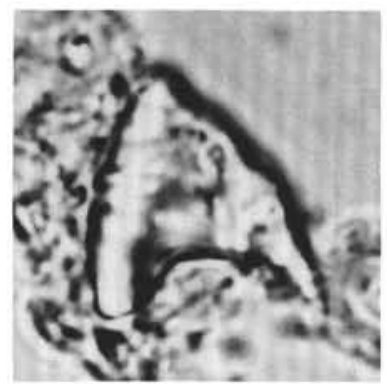

$5 \mathrm{~A}$

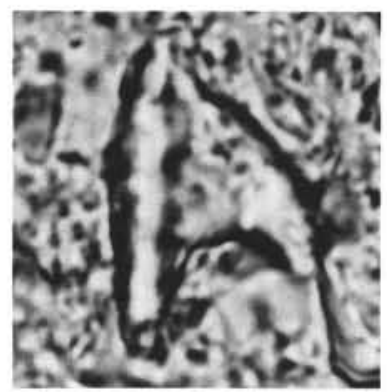

8

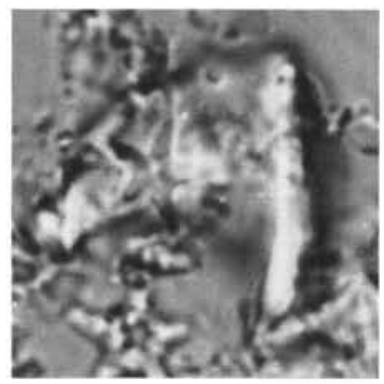

$12 \mathrm{~A}$

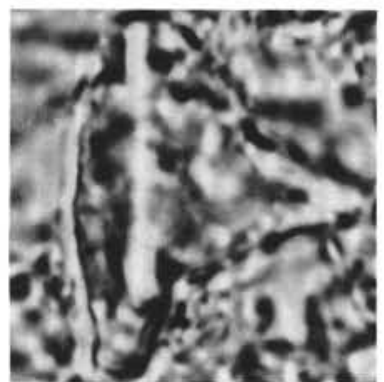

2

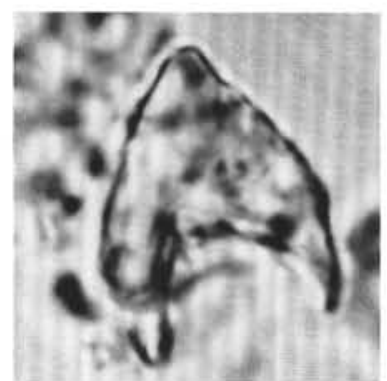

$5 B$

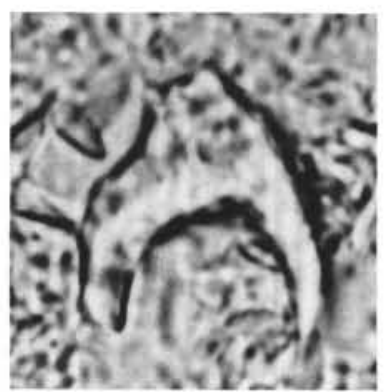

9

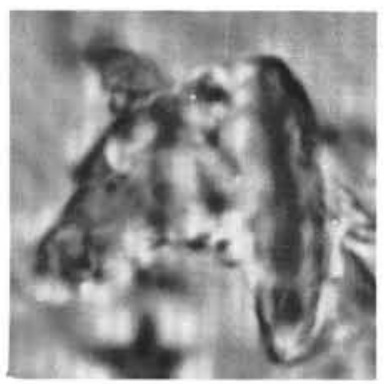

$12 B$

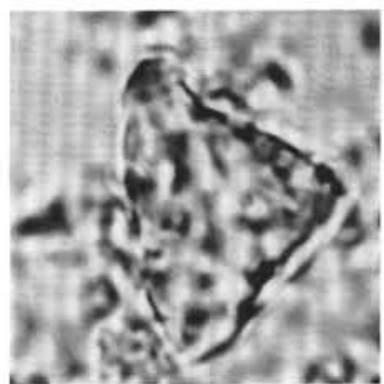

3

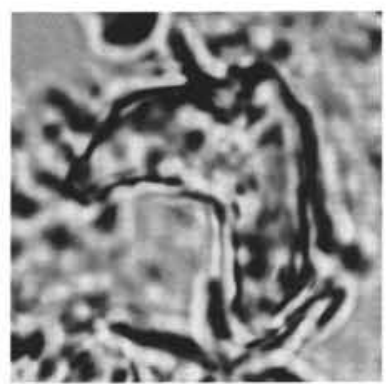

6

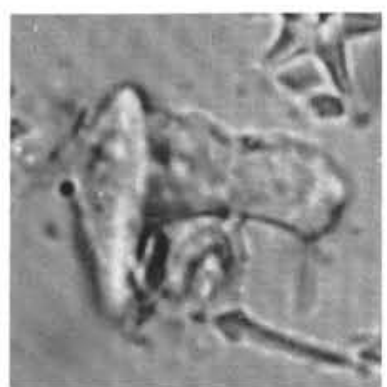

10

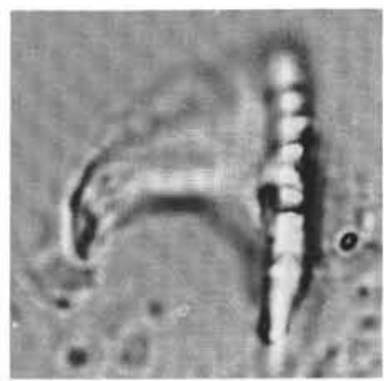

13

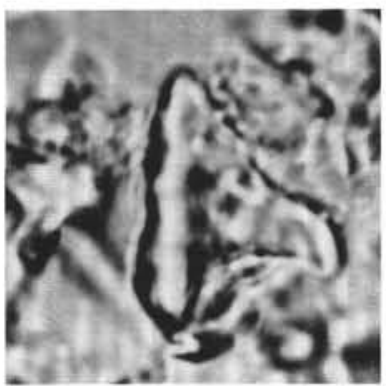

4

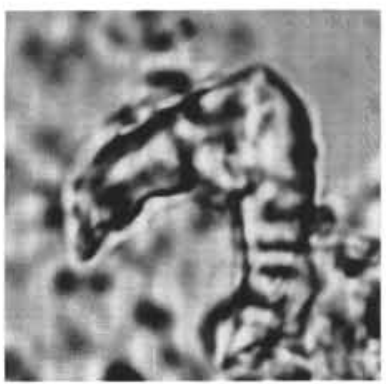

7

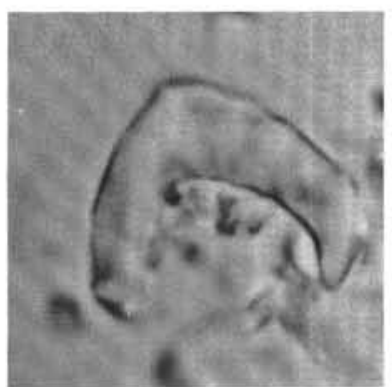

11

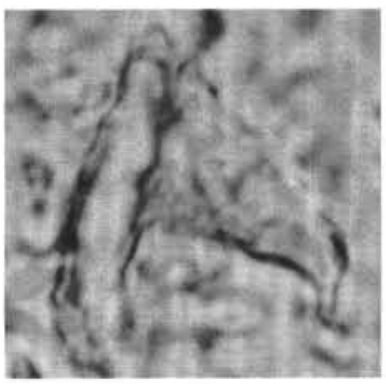

14

Plate 8. All specimens $\times 2400$. 1-3. Triquetrorhabdulus cf. extensus Theodoridis. (1) Sample 115-713A-4R-6, 130 cm; (2) and (3) Sample 115713A-4R-6, $50 \mathrm{~cm}$. 4-6, 8, 10. Triquetrorhabdulus extensus-Amaurolithus amplificus intergrade. (4) Sample 115-713A-4R-3, 130 cm; (5a) Sample 115-713A-4R-6, $130 \mathrm{~cm}$; (5b) different focus of previous sample; (6) Sample 115-713A-4R-4, $130 \mathrm{~cm}$; (8) Sample 115-713A-4R-4, 50 cm; (10) Sample 115-710B-6H-6, $30 \mathrm{~cm}$. 7, 9, 11-14. Amaurolithus amplificus (Bukry and Percival). (7) Sample 115-713A-4R-4, 130 cm; (9) Sample 115-713A-4R-2, $130 \mathrm{~cm}$; (11) Sample 115-710B-6H-4, $130 \mathrm{~cm}$; (12a) Sample 115-710B-6H-6, $110 \mathrm{~cm}$; (12b) different focus of previous sample; (13) Sample 115-711B$4 \mathrm{H}-4,150 \mathrm{~cm}$; (14) Sample 115-709C-9H-6, $60 \mathrm{~cm}$. 


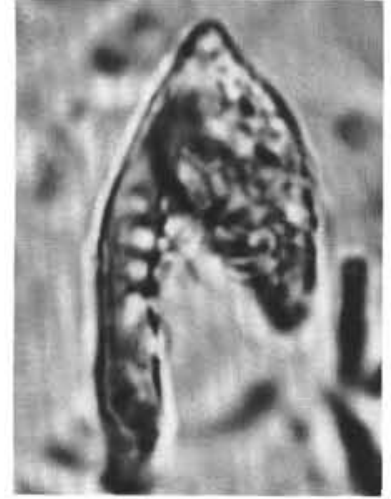

$1 \mathrm{~A}$

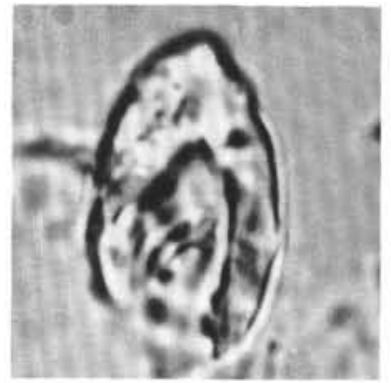

\section{A}

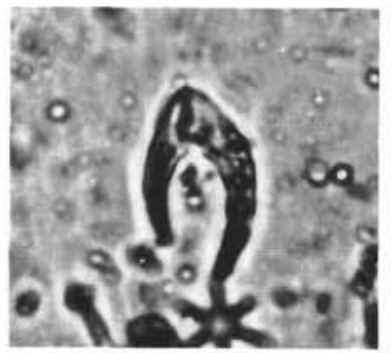

$5 \mathrm{~A}$

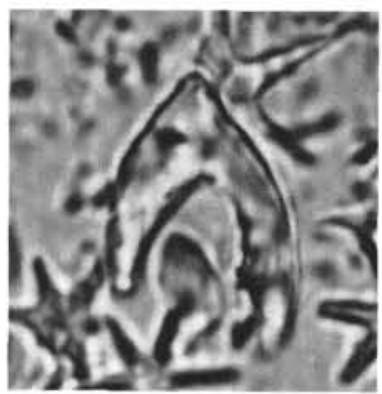

$7 \mathrm{~A}$

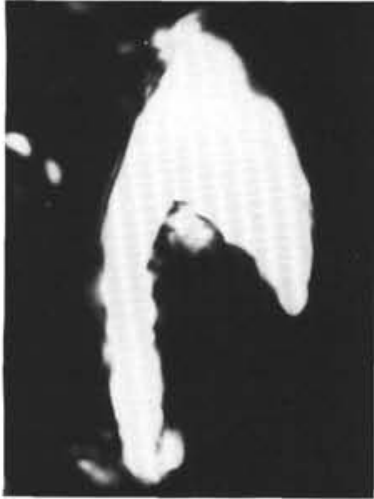

1B

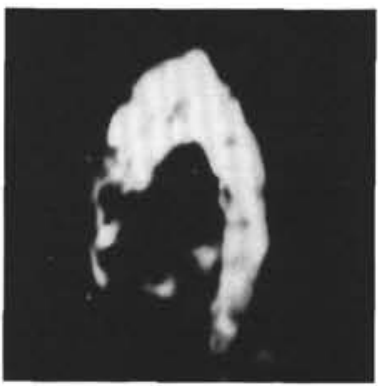

$3 \mathrm{~B}$

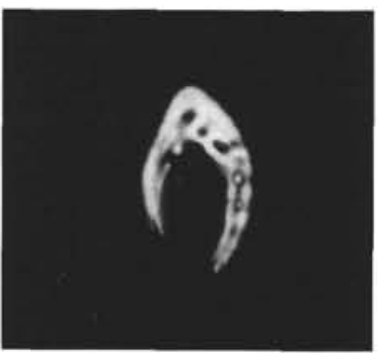

$5 B$

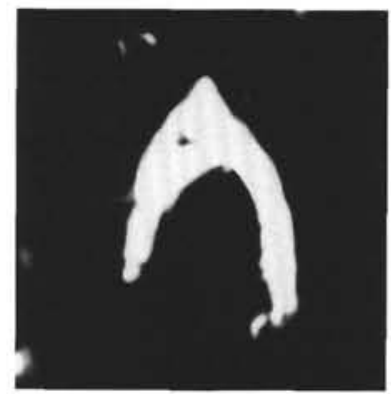

7 B

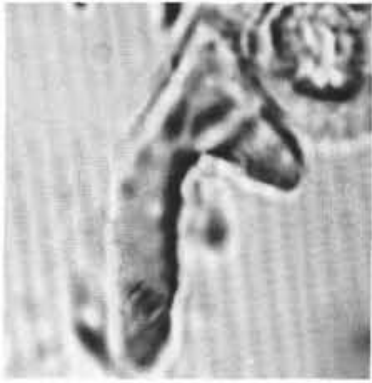

$2 \mathrm{~A}$

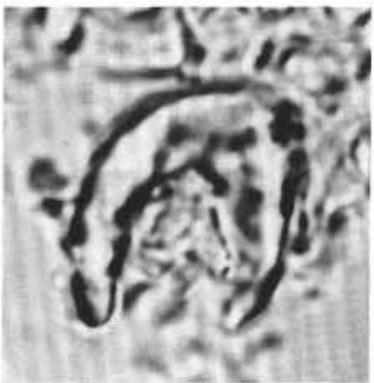

$4 A$

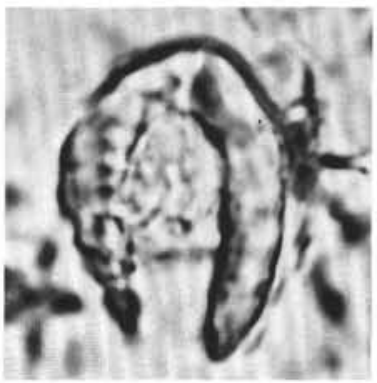

$6 A$

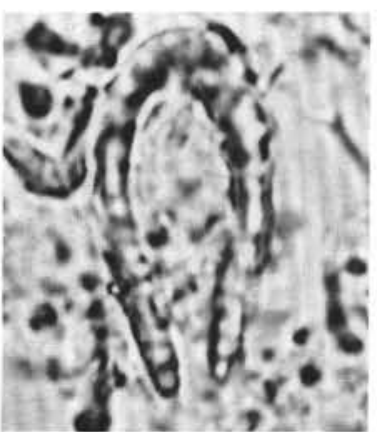

8A

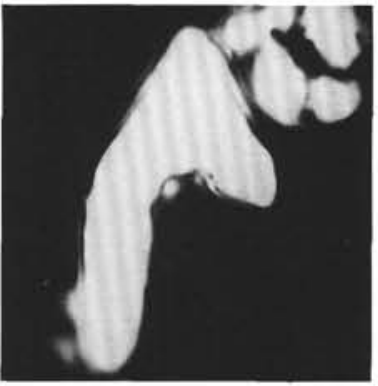

$2 \mathrm{~B}$

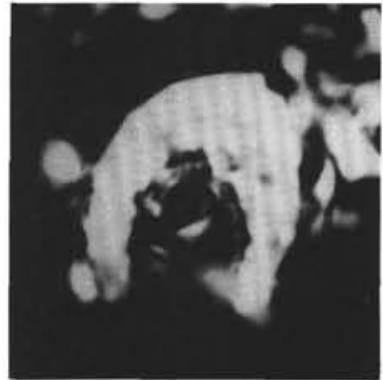

4B

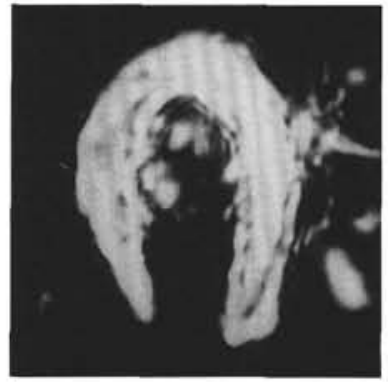

6B

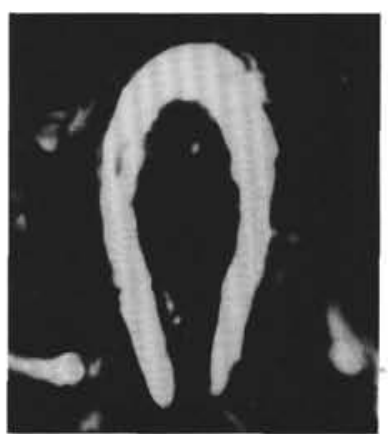

8 B

Plate 9. All specimens $\times 2400$. 1, 2. Ceratolithus acutus Gartner and Bukry. Sample 115-710B-5H-4, $90 \mathrm{~cm}$. (1a) and (2a) Parallel light; (1b) and (2b) crossed nicols. 3. Ceratolithus rugosus-Ceratolithus armatus intergrade. Sample 115-710B-5H-4, $90 \mathrm{~cm}$. (3a) Parallel light; (3b) crossed nicols. 4. Ceratolithus rugosus Bukry and Bramlette. Sample 115-710B-5H-1, $120 \mathrm{~cm}$. (4a) Parallel light; (4b) crossed nicols. 5. Ceratolithus armatus Müller. Sample 115-711B-3H-5, $100 \mathrm{~cm}$. (5a) Parallel light; (5b) crossed nicols. 6. Ceratolithus cristatus Kamptner. Sample 115-710B-4H-3, 60 cm. (6a) Parallel light; (6b) crossed nicols. 7. Ceratolithus acutus-Ceratolithus rugosus intergrade. Sample 115-710B-5H-4, 90 cm. (7a) Parallel light; (7b) crossed nicols. 8. Ceratolithus telesmus Norris. Sample 115-710B-4H-2, $90 \mathrm{~cm}$. (8a) Parallel light; (8b) crossed nicols. 


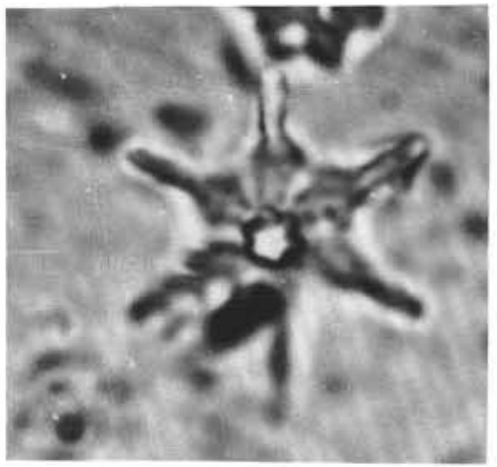

1

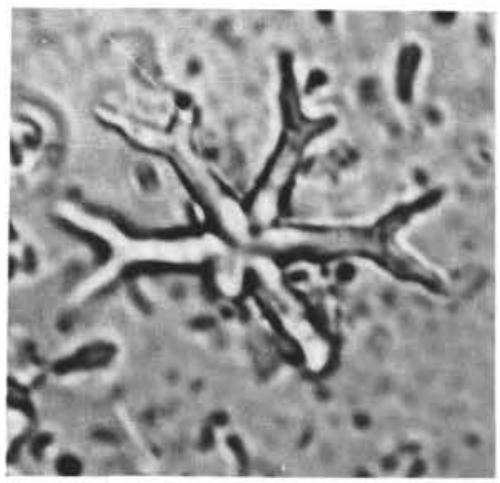

4

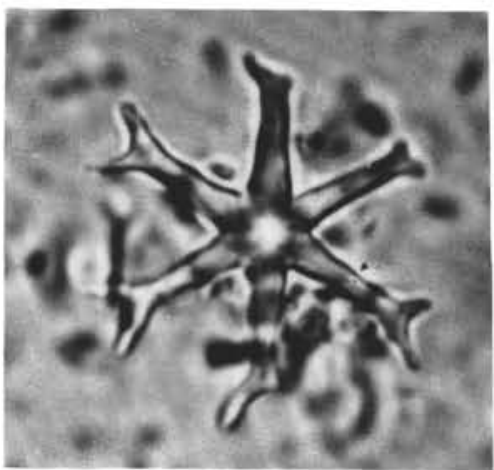

7

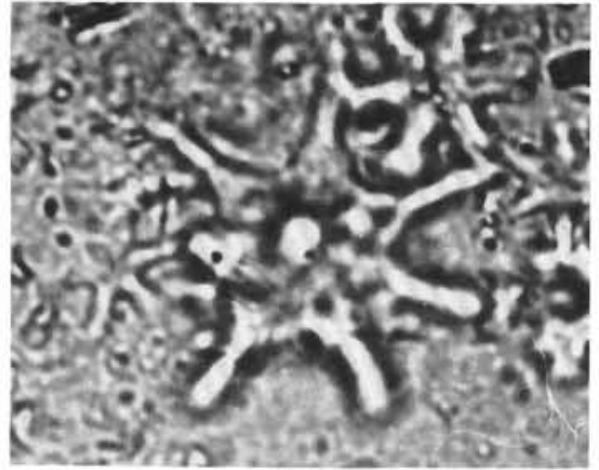

2

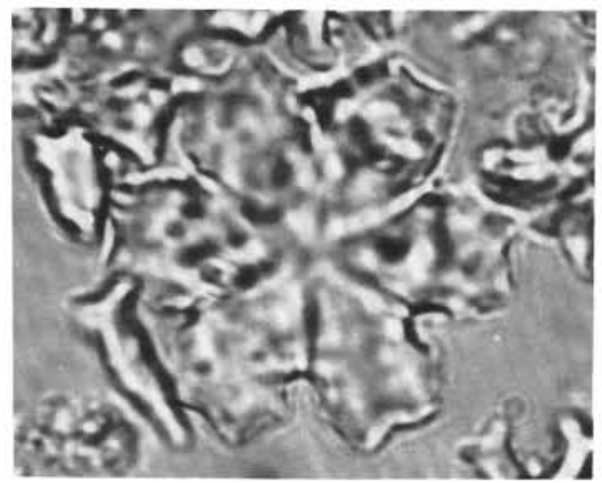

5

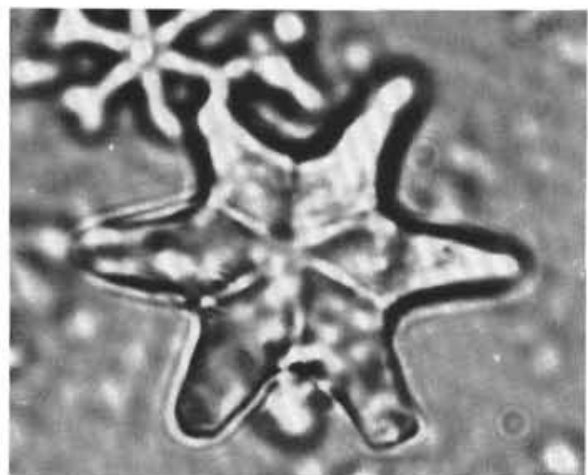

8

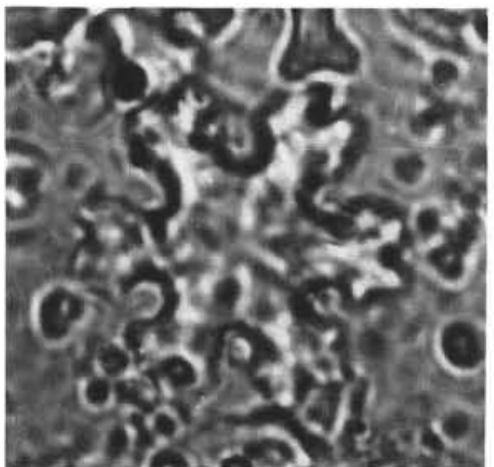

3

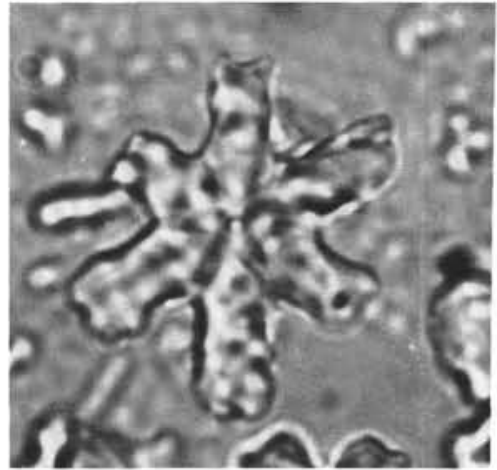

6

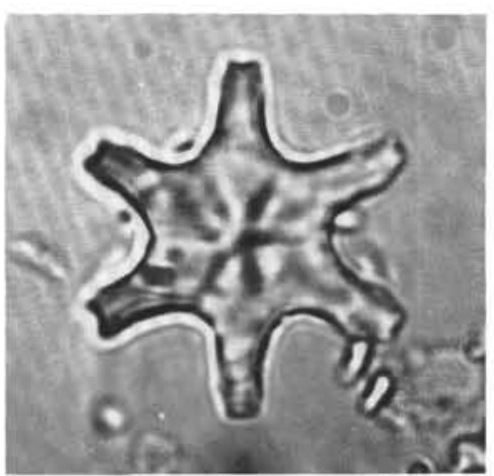

9

Plate 10. All specimens $\times 2400$. 1, 2. Discoaster formosus Martini and Worsley. Sample 115-711B-7H-2, $109 \mathrm{~cm}$. 3. Discoaster deflandrei Bramlette and Riedel. Sample 115-711B-7H-2, 109 cm. 4, 7. Discoaster signus Bukry. Sample 115-711B-6H-6, 140 cm. 5, 6, 8, 9. Discoaster druggii Bramlette and Wilcoxon. Sample 115-710A-13H-2, $30 \mathrm{~cm}$. 


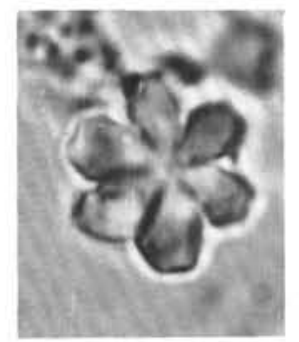

1

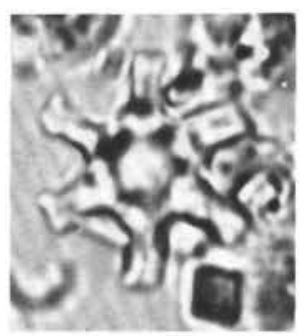

$6 \mathrm{~A}$

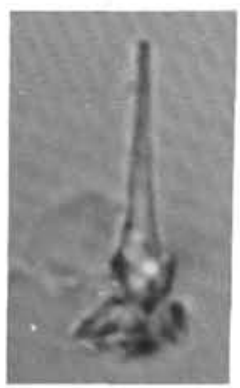

10A

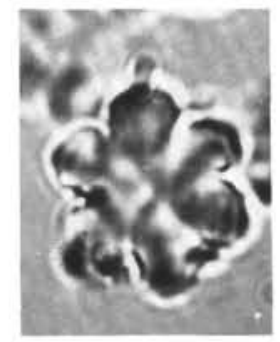

2

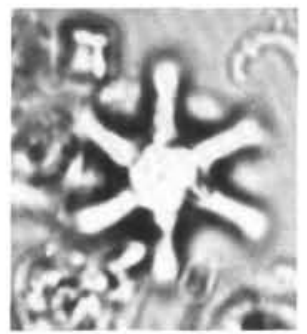

$6 \mathrm{~B}$

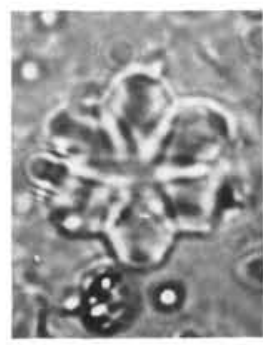

3

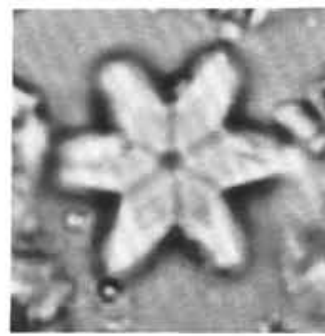

4

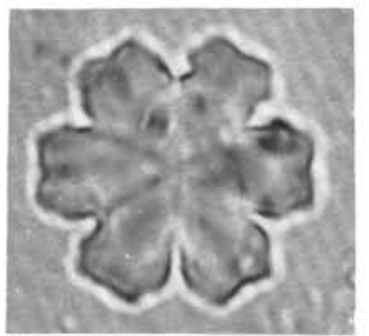

5

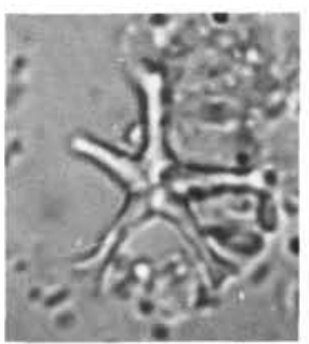

7

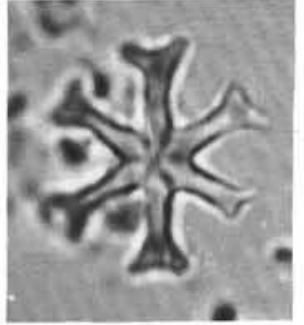

8

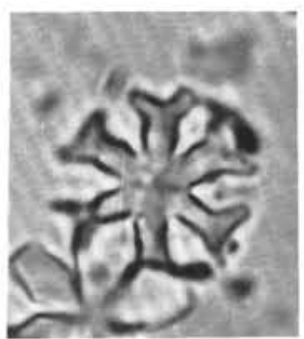

9

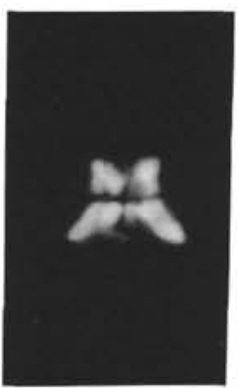

10B

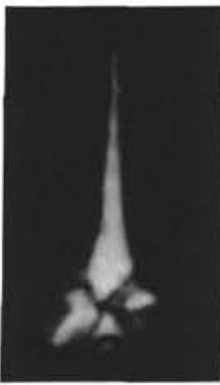

$10 \mathrm{C}$

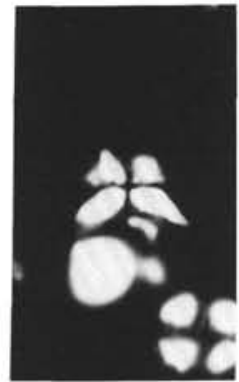

11A

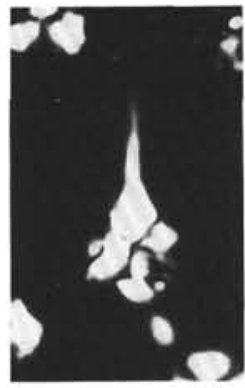

11B

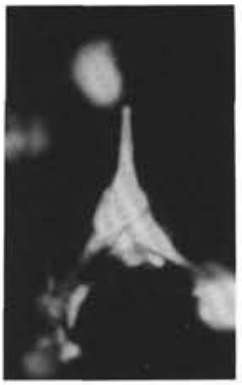

12

Plate 11. All specimens $\times 2400$. 1-3. Discoaster woodringii Bramlette and Riedel. (1) Sample 115-711B-7H-2, 150 cm; (2) Sample 115-711B-9H-1, $80 \mathrm{~cm}$; (3) Sample 115-710A-15X-5, $30 \mathrm{~cm}$. 4, 5. Discoaster aff. druggii Bramlette and Wilcoxon. (4) Sample 115-711B-8H-4, $100 \mathrm{~cm}$; (5) Sample 115-710A-13H-2, $30 \mathrm{~cm}$. 6. Discoaster musicus Stradner. (6a) Sample 115-710A-10H-6, $30 \mathrm{~cm}$; (6b) different focus of previous sample. 7. Discoaster moorei Bukry. Sample 115-711B-7H-2, $150 \mathrm{~cm}$. 8, 9. Discoaster dilatus-extensus group. Sample 115-711B-7H-2, 150 cm. 10-12. Sphenolithus delphix Bukry. Sample 115-714A-21X-CC. (10a) Parallel light, long axis $0^{\circ}$; (10b) and (11a) crossed nicols, long axis $0^{\circ}$; (10c), (11b), and (12) crossed nicols, long axis $45^{\circ}$. 


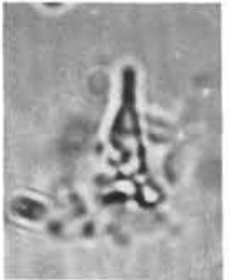

$1 \mathrm{~A}$

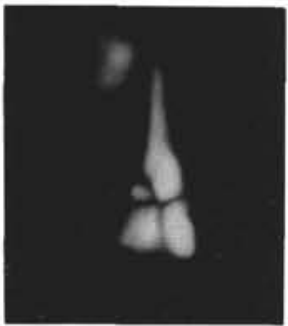

3A

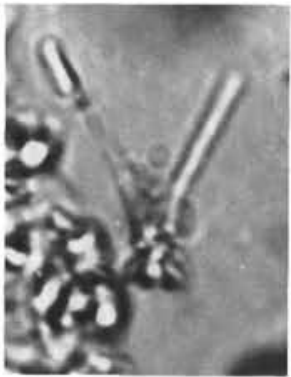

$5 A$

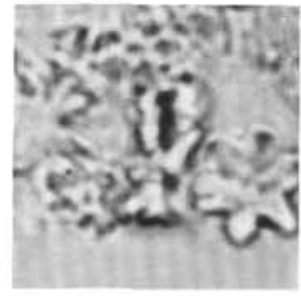

$7 A$

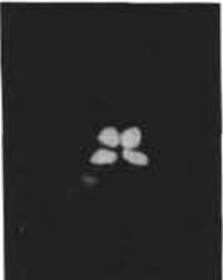

$1 B$

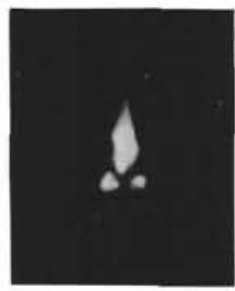

$1 \mathrm{C}$

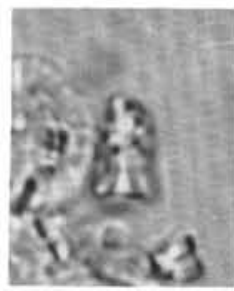

2A

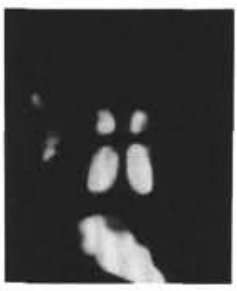

2B

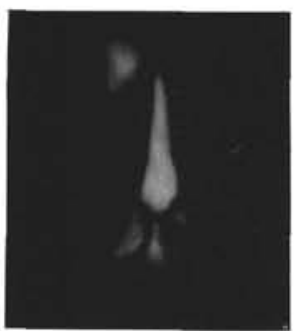

$3 \mathrm{C}$

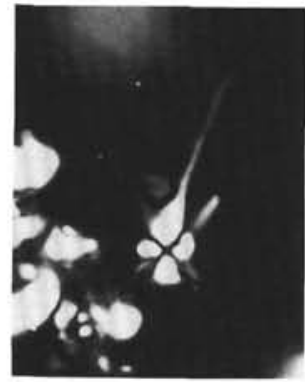

$5 C$

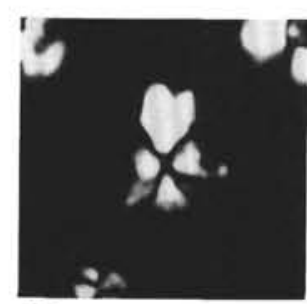

$7 \mathrm{C}$

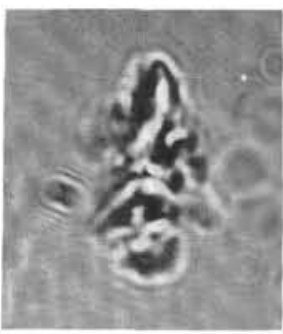

4A

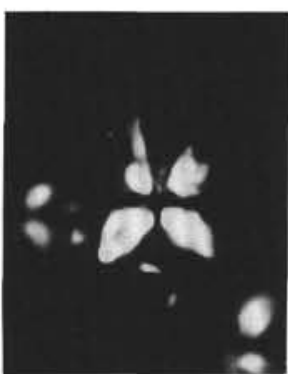

$6 A$

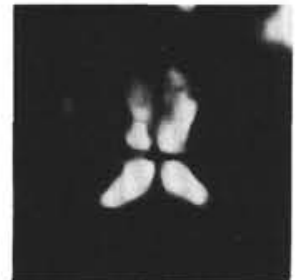

$8 \mathrm{~A}$

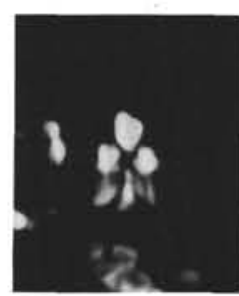

$2 \mathrm{C}$

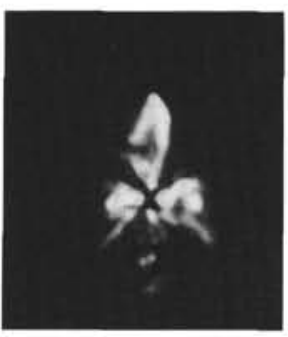

4B

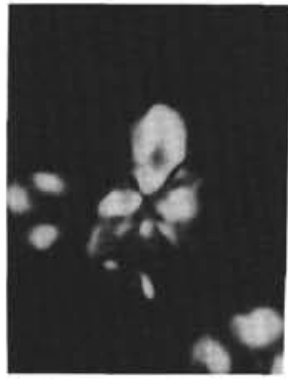

$6 B$

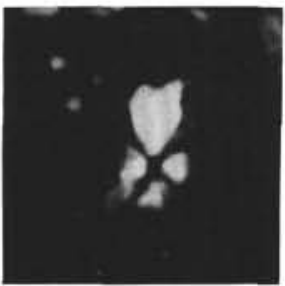

$8 B$

Plate 12. All specimens $\times 2400$. 1. Sphenolithus heteromorphus Deflandre. Sample $115-714 \mathrm{~A}-13 \mathrm{H}-5,75 \mathrm{~cm}$. (1a) Parallel light, long axis $0^{\circ}$; (1b) crossed nicols, long axis $0^{\circ}$; and (1c) crossed nicols, long axis $45^{\circ} .2$. Sphenolithus dissimilis-Sphenolithus belemnos intergrade. Sample 115-710A$14 \mathrm{X}-3,130 \mathrm{~cm}$. (2a) Parallel light, long axis $0^{\circ}$; (2b) crossed nicols, long axis $0^{\circ}$; and $(2 \mathrm{c})$ crossed nicols, long axis $45^{\circ}$. 3. Sphenolithus belemnos Bramlette and Wilcoxon. Sample 115-714A-19X-3, $130 \mathrm{~cm}$. (3a) Crossed nicols, long axis $20^{\circ}$; (3b) crossed nicols, long axis $0^{\circ}$; and (3c) crossed nicols, long axis $45^{\circ}$. 4, 6. Sphenolitus conicus Bukry. Sample 115-714A-22X-1, $130 \mathrm{~cm}$. (4a) Parallel light, long axis $0^{\circ}$; (4b) and $(6 \mathrm{~b}) \mathrm{crossed}$ nicols, long axis $45^{\circ}$; and (6a) crossed nicols, long axis $0^{\circ}$. 5. Sphenolithus capricornutus Bukry and Percival. Sample 115-714A-21X-CC. (5a) Parallel light, long axis $0^{\circ} ;(5 \mathrm{~b})$ crossed nicols, long axis $0^{\circ}$; and $(5 \mathrm{c})$ crossed nicols, long axis $45^{\circ}$. 7, 8. Sphenolithus dissimilis Bukry and Percival. Sample 115-709C-15H-6, $60 \mathrm{~cm}$. (7a) Parallel light, long axis $0^{\circ}$; (7b) and (8a) crossed nicols, long axis $0^{\circ}$; (7c) and (8b) crossed nicols, long axis $45^{\circ}$. 


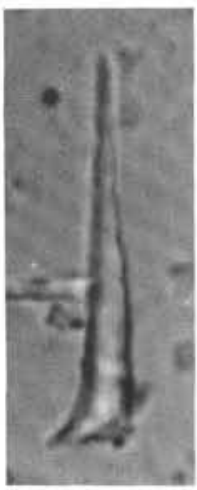

$1 \mathrm{~A}$

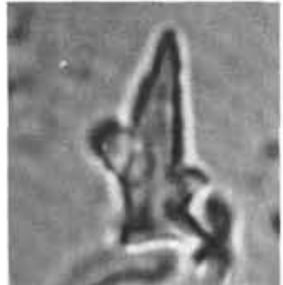

4

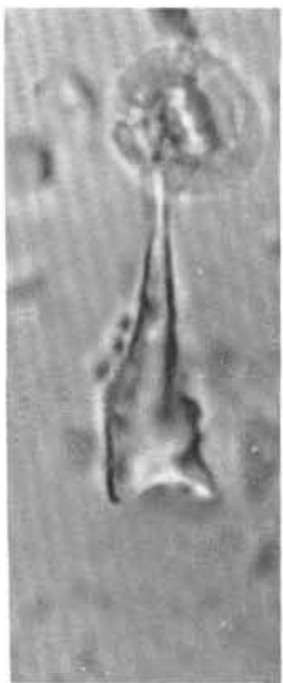

7

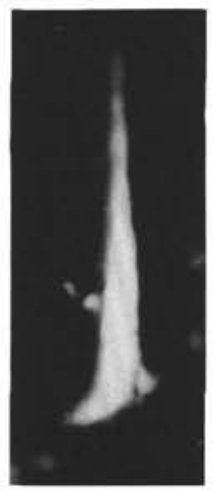

1B

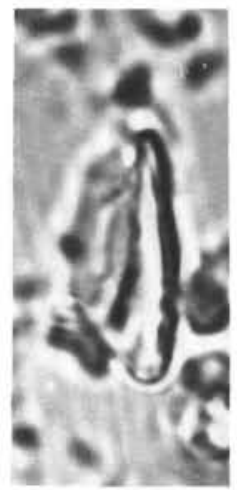

$2 \mathbf{A}$

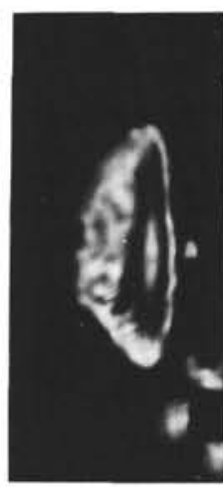

$2 B$

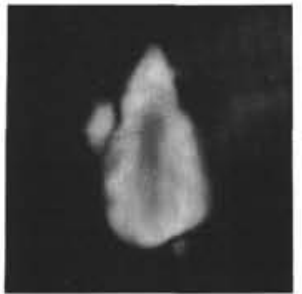

5B

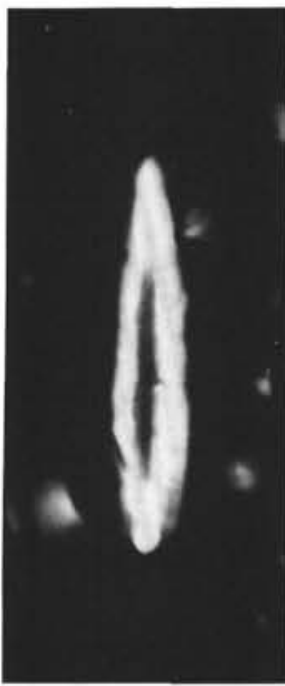

$8 B$

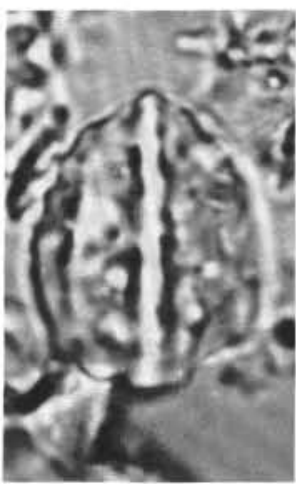

3A

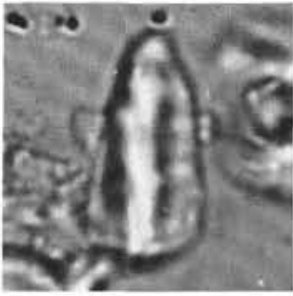

6A

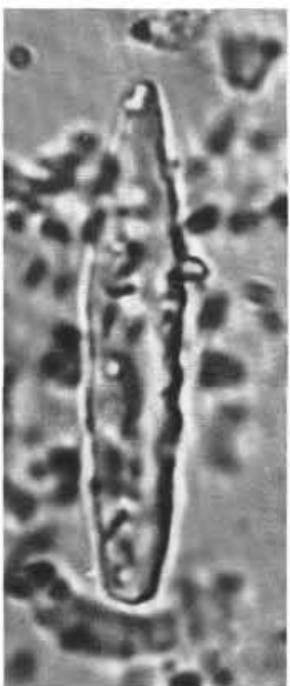

9A

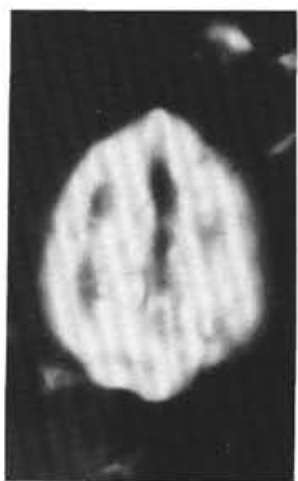

3B

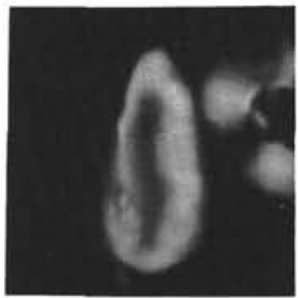

$6 B$

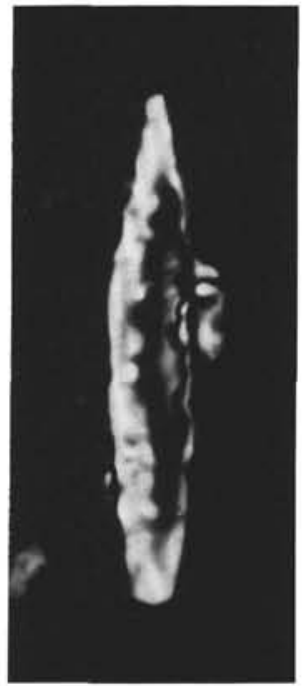

9B

Plate 13. All specimens $\times 2400$. 1. Triquetrorhabdulus serratus (Bramlette and Wilcoxon). Sample $115-710 \mathrm{~A}-10 \mathrm{H}-3,30 \mathrm{~cm}$. (1a) Parallel light, long axis $90^{\circ}$; (1b) crossed nicols, long axis $45^{\circ}$. 2. Triquetrorhabdulus milowii Bukry. Sample $115-709 \mathrm{C}-17 \mathrm{H}-3,30 \mathrm{~cm}$. (2a) Parallel light, long axis $0^{\circ}$; (2b) crossed nicols, long axis $45^{\circ}$. 3. Triquetrorhabdulus challengeri Perch-Nielsen. Sample $115-711 \mathrm{~B}-8 \mathrm{H}-4,100 \mathrm{~cm}$. (3a) Parallel light, long axis $0^{\circ}$; (3b) crossed nicols, long axis $45^{\circ}$. 4, 7. Triquetrorhabdulus cf. serratus (Bramlette and Wilcoxon). Sample 115-710A-10H-3, 30 cm. Parallel light, long axis $0^{\circ}$. 5, 6. Triquetrorhabdulus auritus Stradner and Allram. Sample 115-710A-13H-2, $130 \mathrm{~cm}$. (5a) and (6a) Parallel light, long axis $0^{\circ}$; (5b) and (6b) crossed nicols, long axis $45^{\circ}$. 8, 9. Triquetrorhabdulus carinatus Martini. Sample 115-711B-9H-2, 50 cm. (8a) and (9a) Parallel light, long axis $0^{\circ} ;(8 \mathrm{~b})$ and $(9 \mathrm{~b})$ crossed nicols, long axis $45^{\circ}$. 


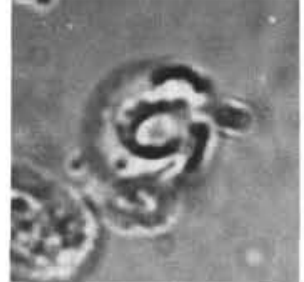

$1 \mathrm{~A}$

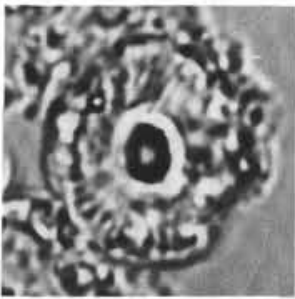

4A

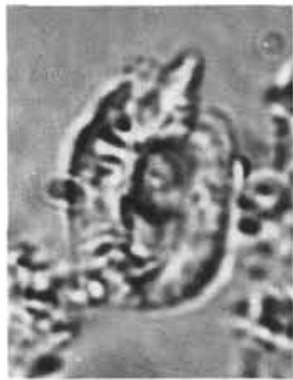

$8 \mathrm{~A}$

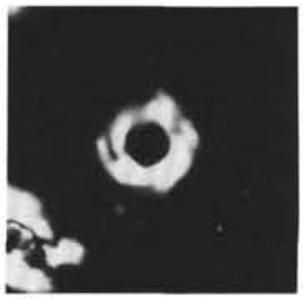

$1 B$

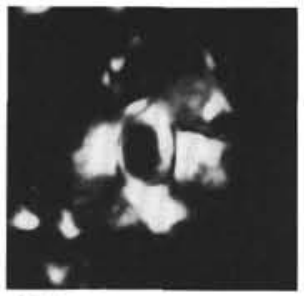

4B

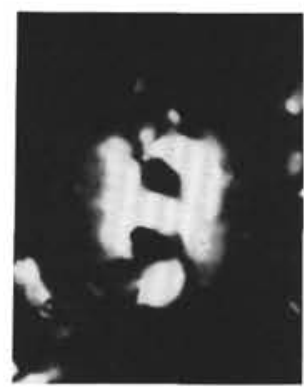

$8 B$

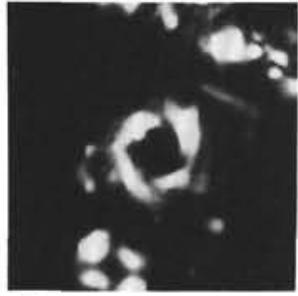

2

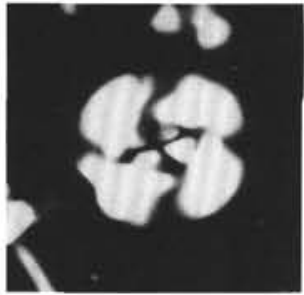

5

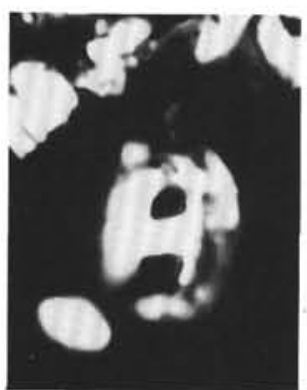

9

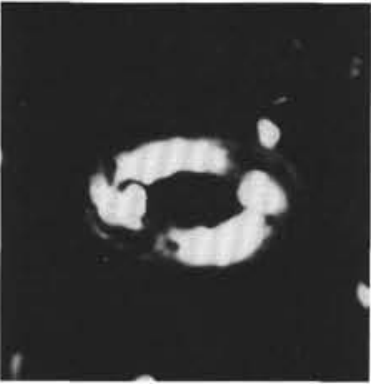

11B

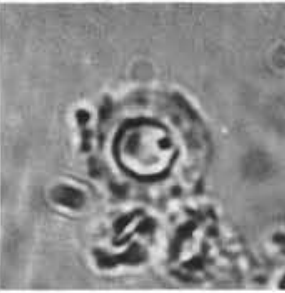

$3 \mathbf{A}$

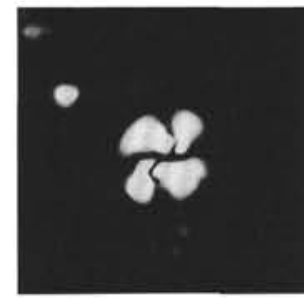

6

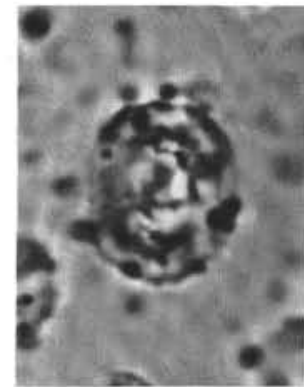

$10 \mathrm{~A}$

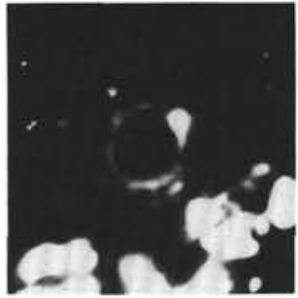

3B

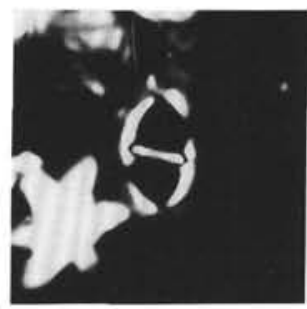

7

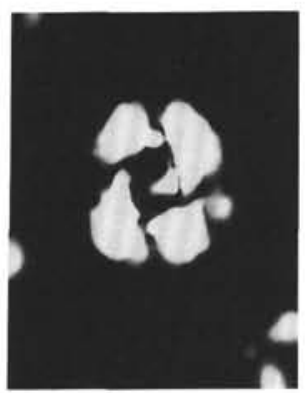

$10 B$

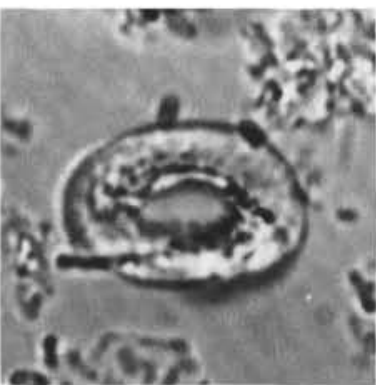

$11 \mathrm{~A}$

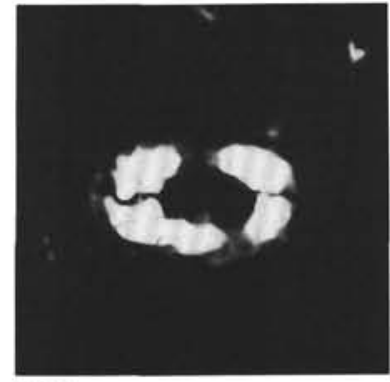

$11 \mathrm{C}$

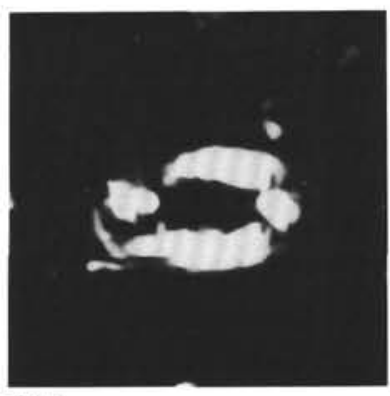

11D

Plate 14. All specimens $\times 2400$. 1, 2. Hayella aperta Theodoridis. Sample 115-714A-21X-CC. (1a) Parallel light; (1b) and (2) crossed nicols. 3. Geminilithella rotula (Kamptner). Sample 115-714A-13H-4, $75 \mathrm{~cm}$. (3a) Parallel light; (3b) crossed nicols. 4. Calcidiscus premacintyrei Theodoridis. Sample 115-714A-13H-3, $75 \mathrm{~cm}$. (4a) Parallel light; (4b) crossed nicols. 5. Reticulofenestra daviesii (Haq). Sample 115-715A-10R-3, 130 cm. Crossed nicols. 6. Dictyococcites sp. Black. Sample 115-714A-13H-4, $75 \mathrm{~cm}$. Crossed nicols. 7. Solidopons petrae Theodoridis. Sample 115$715 \mathrm{~A}-9 \mathrm{R}-1,130 \mathrm{~cm}$. Crossed nicols. 8, 9. Helicosphaera recta Haq. Sample 115-714A-15X-3, $75 \mathrm{~cm}$. (8a) Parallel light, long axis $0^{\circ}$; (8b) and (9) crossed nicols, long axis $0^{\circ}$. 10. Reticulofenestra aff. pseudoumbilicus. Sample 115-708A-16X-6, $91 \mathrm{~cm}$. (10a) Parallel light; (10b) crossed nicols. 11. Helicosphaera ampliaperta Bramlette and Wilcoxon. Sample $115-714 \mathrm{~A}-14 \mathrm{X}-5,75 \mathrm{~cm}$. (11a) Parallel light, long axis $0^{\circ}$; (11b) crossed nicols, long axis $0^{\circ}$; (11c) crossed nicols, long axis $15^{\circ}$; and (11d) crossed nicols, long axis $52^{\circ}$. 